\title{
Measuring the effects of HPV16/18 vaccination on HPV positivity and anogenital warts
}

Citation for published version (APA):

Woestenberg, P. (2020). Measuring the effects of HPV16/18 vaccination on HPV positivity and anogenital warts. [Doctoral Thesis, Maastricht University]. ProefschriftMaken.

https://doi.org/10.26481/dis.20200930pw

Document status and date:

Published: 01/01/2020

DOI:

10.26481/dis.20200930pw

Document Version:

Publisher's PDF, also known as Version of record

\section{Please check the document version of this publication:}

- A submitted manuscript is the version of the article upon submission and before peer-review. There can be important differences between the submitted version and the official published version of record.

People interested in the research are advised to contact the author for the final version of the publication, or visit the DOI to the publisher's website.

- The final author version and the galley proof are versions of the publication after peer review.

- The final published version features the final layout of the paper including the volume, issue and page numbers.

Link to publication

\footnotetext{
General rights rights.

- You may freely distribute the URL identifying the publication in the public portal. please follow below link for the End User Agreement:

www.umlib.nl/taverne-license

Take down policy

If you believe that this document breaches copyright please contact us at:

repository@maastrichtuniversity.nl

providing details and we will investigate your claim.
}

Copyright and moral rights for the publications made accessible in the public portal are retained by the authors and/or other copyright owners and it is a condition of accessing publications that users recognise and abide by the legal requirements associated with these

- Users may download and print one copy of any publication from the public portal for the purpose of private study or research.

- You may not further distribute the material or use it for any profit-making activity or commercial gain

If the publication is distributed under the terms of Article $25 \mathrm{fa}$ of the Dutch Copyright Act, indicated by the "Taverne" license above, 


\section{Measuring the effects of HPV16/18 vaccination on HPV positivity and anogenital warts}

\section{Citation for published version (APA):}

Woestenberg, P. J. (2020). Measuring the effects of HPV16/18 vaccination on HPV positivity and anogenital warts. ProefschriftMaken. https://doi.org/10.26481/dis.20200930pw

Document status and date:

Published: 01/01/2020

DOI:

10.26481/dis.20200930pw

Document Version:

Publisher's PDF, also known as Version of record

\section{Please check the document version of this publication:}

- A submitted manuscript is the version of the article upon submission and before peer-review. There can be important differences between the submitted version and the official published version of record.

People interested in the research are advised to contact the author for the final version of the publication, or visit the DOI to the publisher's website.

- The final author version and the galley proof are versions of the publication after peer review.

- The final published version features the final layout of the paper including the volume, issue and page numbers.

Link to publication

\footnotetext{
General rights rights.

- You may freely distribute the URL identifying the publication in the public portal. please follow below link for the End User Agreement:

www.umlib.nl/taverne-license

Take down policy

If you believe that this document breaches copyright please contact us at:

repository@maastrichtuniversity.nl

providing details and we will investigate your claim.
}

Copyright and moral rights for the publications made accessible in the public portal are retained by the authors and/or other copyright owners and it is a condition of accessing publications that users recognise and abide by the legal requirements associated with these

- Users may download and print one copy of any publication from the public portal for the purpose of private study or research.

- You may not further distribute the material or use it for any profit-making activity or commercial gain

If the publication is distributed under the terms of Article 25fa of the Dutch Copyright Act, indicated by the "Taverne" license above, 


\section{Measuring the effects of HPV16/18 vaccination on HPV positivity and anogenital warts}

Petra Woestenberg 
Measuring the effects of HPV16/18 vaccination on HPV positivity and anogenital warts

(C) 2020 Petra Woestenberg

ISBN: 978-94-6380-896-5

Printed by: ProefschriftMaken | www.proefschriftmaken.nl

The studies described in this thesis were financially supported by the Dutch Ministry of Health, Welfare and Sport.

All rights reserved. No part of this thesis may be reproduced, stored, or transmitted in any form or by any means, electronic or mechanical, including photocopy, recording or any information storage or retrieval system, without prior permission of the copyright owner. 


\title{
Measuring the effects of HPV16/18 vaccination on HPV positivity and anogenital warts
}

\author{
PROEFSCHRIFT \\ ter verkrijging van de graad van doctor aan de Universiteit Maastricht, \\ op gezag van de Rector Magnificus, Prof. dr. Rianne M. Letschert \\ volgens besluit van het College van Decanen, \\ in het openbaar te verdediging
}

op woensdag 30 september 2020 om 13:00 uur

door

Petra Johanna Woestenberg

Geboren op 12 mei 1989 te Alphen en Riel 


\section{Promotor}

Prof. dr. C.J.P.A Hoebe

\section{Copromotoren}

Dr. J.A. Bogaards, RIVM Bilthoven

Dr. B.H.B. van Benthem, RIVM Bilthoven

\section{Beoordelingscommissie}

Prof. dr. J.W.L. Cals (voorzitter)

Prof. dr. P.H.M. Savelkoul

Prof. dr. M.F. Schim van der Loeff, Universiteit van Amsterdam

Prof. dr. M.E.E. Kretzschmar, Universiteit Utrecht 
The research presented in this thesis was conducted at CAPHRI Care and Public Health Research Institute, Department FHML, of Maastricht University. CAPHRI participates in the Netherlands School of Public Health and Care Research CaRe. 


\section{Table of content}

Chapter $1 \quad$ General introduction

\section{Part 1: Direct vaccine effectiveness against HPV positivity}

Chapter 2 Bivalent vaccine effectiveness against type-specific HPV

positivity: Evidence for cross-protection against oncogenic types among Dutch STI clinic visitors

J Infect Dis. 2018; 217:213-22

Chapter 3 Bivalent human papillomavirus (HPV) vaccine effectiveness correlates with phylogenetic distance from HPV vaccine types 16 and 18

J Infect Dis. 2019; 220:1141-6

Chapter $4 \quad$ Bivalent vaccine effectiveness against anal human

61 papillomavirus positivity among female sexually transmitted infection clinic visitors in the Netherlands J Infect Dis. 2020; 221:1280-5

Part 2: Population-level impact on HPV positivity

Chapter 5

Assessment of herd effects among women and heterosexual men after girls-only HPV16/18 vaccination in the Netherlands: A repeated cross-sectional study Int J Cancer. 2019; 144:2718-27

Chapter 6 Population impact of girls-only HPV16/18 vaccination in the Netherlands: Cross-protective and second-order herd effects Chapter 7 HPV infections among young MSM visiting sexual health centers in the Netherlands: Opportunities for targeted HPV vaccination Vaccine. 2020; 38:3321-9

Part 3: Direct vaccine effectiveness against anogenital warts against HPV6/11 positivity in female STI clinic visitors J Infect. 2017; 74:393-400 
Chapter 9

Partial protective effect of bivalent HPV16/18 vaccination

against anogenital warts in a large cohort of Dutch primary care patients

Clin Infect Dis. 2020; 17:ciaa582

Chapter $10 \quad$ General discussion

189

Supplement

Summary 210

Nederlandse samenvatting

213

Valorization

216

List of abbreviations

220

Dankwoord

222

Curriculum vitae

225

List of publications

226 



\title{
Chapter 1
}

\author{
General introduction
}




\section{Overview}

Sexually transmitted human papillomavirus (HPV) is usually asymptomatic but can cause anogenital warts (AGWs) and various anogenital and head-and-neck cancers in both men and women. The most important types causing cancer are HPV16 and HPV18 and the most important types causing AGWs are HPV6 and HPV11. To prevent HPV infection and associated diseases, prophylactic vaccines have been developed. In the Netherlands, free of charge HPV vaccination was introduced in 2009 for girls only, using the bivalent HPV vaccine that targets HPV16 and HPV18. The program started with a catch-up campaign for 12- to 16-year-old girls born in 1993-1996. From 2010 onwards, HPV vaccination was offered to girls in the year they turn 13 years old. After implementation of vaccines in national immunization programs, post-implementation studies are important to assess the (long-term) effects of vaccination. Multiple vaccine effects can be distinguished, like the direct vaccine effectiveness and the impact of the vaccination program in the population. Because vaccination changes the force of infection, evaluation of these vaccine effects needs to take some methodological challenges into account.

The goal of this thesis is to measure effects of the bivalent HPV vaccine and the HPV vaccination program in the Netherlands. Because it takes decades for HPV infections to cause cancer, we focus on the surrogate end-point HPV positivity and AGWs for which it takes less time to assess vaccine effects. Focusing on HPV infections will also provide unique insights into the HPV infection dynamics after vaccine introduction. In the following sections of this introductory chapter we will give a brief introduction to HPV, HPV-related diseases, the available HPV vaccines, and the implementation of HPV vaccination in the Netherlands. Moreover, we will describe the different effects of vaccination that can be distinguished and how these effects can be measured using a theoretical framework. We will end with the outline of this thesis.

\section{HPV and HPV-related diseases}

$\mathrm{HPV}$ is a small, double-stranded, non-enveloped, circular DNA virus with 8 protein-coding genes ( 6 early genes E1, E2, E4, E5, E6, and E7 that encode proteins involved in replication, transcription, and immune suppression; and 2 late genes L1 and L2 that encode capsid proteins) and a noncoding, regulatory long control region (LCR) [1]. Currently, there are over 200 different HPV types identified, divided into the genera alpha, beta, gamma, mu, and nu. We will focus on the HPV types of the alpha genus that can infect the mucosal epithelia and are transmitted through sexual contact. There are about 50 different mucosal $\alpha$ HPV types, which differ by definition 10-30\% in the L1 gene sequence [2-5]. The HPV types are generally classified as high-risk (hr) or low-risk (Ir) HPV types based on their oncogenic potential [6]. 
People get exposed to sexually transmitted HPV via an infected partner. Knowledge of the life-cycle of HPV infections is mostly based on research on cervical HPV16 infection and proceeds as follows [5]. Via micro-abrasions in the mucosa the virus can reach the basal cell layer and infect undifferentiated basal epithelial cells. Using host replication machinery, HPV replicates while the basal cells differentiate. When infected cells reach the epithelial surface the lifecycle of HPV is completed and viral particles are shed. This is called a productive infection [7-9]. In 80\%-90\% of incident infections, HPV will become undetectable within a few years after acquisition, with longer time to become undetectable for HPV16 compared to other hrHPV types [10-14]. It is unknown if the virus is then truly cleared or present in low copy numbers at an undetectable level (latent infection) $[15,16]$. In case an infection with a hrHPV type is not cleared, a subset of the infections can become a transforming infection, meaning that the E6 and E7 genes become deregulated and expressed in dividing basal cells. By interfering with the host cell-cycle, a transforming infection can drive cell proliferation, leading to uncontrolled cell growth (neoplasia) and ultimately invasive cancer, a process that can take decades $[17,18]$. HPV-related cancers are preceded by precancerous lesions. For squamous cell cancers, these so called intraepithelial neoplasia's are divided into stages based on the severity of the dysplasia. For example cervical intraepithelial neoplasia (CIN) are graded from CIN1 (low-grade lesions) to CIN3 (high-grade lesions). Progression and regression to and from each stage is possible, with lower chances to regress with higher grade lesions [19]. It has been estimated that CIN1, CIN2, and CIN3 will progress to cervical cancer in about $1 \%, 5 \%$, and $12 \%-30 \%$ of the cases without treatment respectively $[20,21]$. Progression to cancer at other anatomical sites is less well studied, but seems to be comparable to the cervix. Data suggest that highgrade anal lesions progress to anal cancer in about $9 \%-13 \%$ of the cases within 5 years [22]. The life-cycle of IrHPV types is much less studied, but thought to be broadly comparable to the life-cycle of hrHPV types, with the exception for viral gene expression to become deregulated and the ability to drive cell proliferation in the basal cell layer [5]. Therefore IrHPV types rarely cause neoplasia or invasive cancers. Instead these types, especially IrHPV types 6 and 11, are more often associated with the development of AGWs and the rare condition recurrent respiratory papillomatosis $[23,24]$. The incubation period of AGWs is much shorter compared to cancer and estimated to be between 3-18 months with a median of 6-10 months [25].

HPV is very prevalent and highly transmissible; it is estimated that about $80 \%$ of the sexually active population will acquire a sexually transmitted HPV infection at least once during lifetime [26]. Like other sexually transmitted infections (STIS), the main risk factors to acquire HPV are related to sexual risk behavior, such as the number of vaginal, anal, and oral sex partners. Other risk factors for an HPV infection include factors like smoking 
behavior, oral contraceptive use, and concurrent STIs [27]. Condom use is only partially effective in preventing transmission and most HPV infections occur soon after sexual debut [28-31]. While among women in industrialized countries the peak of genital infection is before the mid-twenties, this is less so the case among men [12, 32-34].

Cancers with an established HPV etiology are cervical, vaginal, vulvar, penile, anal, and oropharyngeal cancer [35]. Almost all cervical cancers are caused by HPV infections and HPV is postulated to be a necessary cause in the cervical cancer development [36, 37]. The HPV attributable fraction for the other cancer sites is lower and depends on the study population and diagnostic measures used [37, 38]. Worldwide, the HPV attributable fraction is estimated to be $25 \%$ for vulvar and up to $88 \%$ for anal cancer (Table 1.1 ). The hrHPV types 16 and 18 are the most import types causing cancer. About $71 \%$ of the cervical cancers are associated with HPV16/18. Other hrHPV types in cervical cancer, in descending order of importance are: $45,33,31,52,58,35,39,51,59$, and 56 [39]. Together the relative attribution of HPV31/33/45/52/58 in cervical cancer is $18 \%$ [40]. The relative attribution of HPV16/18 for other cancers sites is estimated to be 64\% for HPV-related vaginal cancer and up to $87 \%$ and $90 \%$ for HPV-related anal and oropharyngeal cancer respectively (Table 1.1) [40]. AGWs are most commonly caused by IrHPV types 6 and 11 that are estimated to cause $90 \%$ of the cases [24].

Table 1.1. The global HPV attributable fraction and the relative contribution of specific HPV types in HPV-related cancer, per cancer site.

\begin{tabular}{llll}
\hline Cancer site & $\begin{array}{l}\text { HPV attributable } \\
\text { fraction (\%) [37] }\end{array}$ & $\begin{array}{l}\text { Relative attribution of } \\
\text { HPV16/18 in HPV- } \\
\text { related cancer (\%) [40] }\end{array}$ & $\begin{array}{l}\text { Relative attribution of } \\
\text { HPV31/33/45/52/58 in HPV- } \\
\text { related cancer (\%) [40] }\end{array}$ \\
\hline Cervix & 100 & 71 & 18 \\
Anus & 88 & 87 & 8 \\
Oropharynx & 31 & 90 & 5 \\
Vagina & 78 & 64 & 21 \\
Vulva & 25 & 79 & 14 \\
Penis & 50 & 77 & 11 \\
\hline
\end{tabular}

Because HPV is very prevalent, the HPV-related disease burden is high. Worldwide, 570,000 women were diagnosed with cervical cancer in 2018, making cervical cancer the most important HPV-related cancer and the $4^{\text {th }}$ most common cancer in women globally (Figure 1.1) [41]. There were much less, but still a substantial number of HPV-related anal $(43,000)$, oropharyngeal $(29,000)$, vaginal $(14,000)$, vulvar $(11,000)$, and penile $(17,000)$ cancer diagnoses. Oropharyngeal cancer is more often observed in men, while anal cancer is slightly more often observed in women. Among men, having sex with another men is a 
major risk factor for especially anal cancer and to a lesser extent oropharyngeal cancer [42]. Many industrialized countries have observed increases in anal, vulvar, and oropharyngeal cancers in recent years, which has been associated with changes in sexual risk behavior in recent decades [43-45]. For AGWs a reliable number of worldwide diagnoses is lacking. For Europe, the annual number of new AGW diagnoses was estimated to be between 380,000 and 510,000 among women and between 340,000 and 460,000 among men [46].

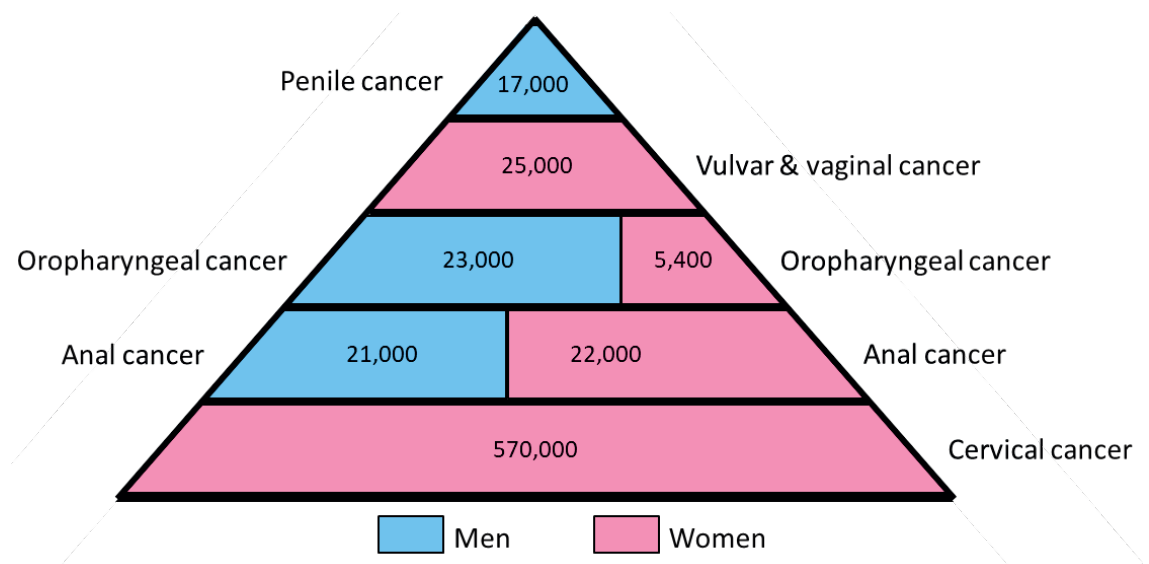

Figure 1.1. Global estimated number of HPV-related cancer diagnoses in 2018 by cancer site and sex. Note: To come to these numbers the HPV attributable fraction and male-female distribution per cancer site were adapted from De Martel et al. 2017 [37] and projected on the number of world-wide new cancers in 2018 retrieved from Globocan 2018 [41]. Numbers are rounded to 2 significant digits.

Also in the Netherlands the HPV-related disease burden is high and higher compared to other infectious diseases. The annual HPV-related disease burden was 13,900 disabilityadjusted life-year (DALY) in the period 2011-2014 [47]. In comparison, disease burden due to influenza was about 10,500 DALY in the period 2013-2017 [48]. In the cervical screening program, women are screened for precancerous lesions and early detection of cervical cancer, and treated if necessary. Since the introduction of screening, the incidence of cervical cancer and the mortality due to the disease have decreased substantially, from 12 to 3.6/100,000 woman-years and from 5.4 to $1.1 / 100,000$ women-years in the period 19702003, respectively [49]. Despite screening, an increase in the incidence of cervical cancer has been observed in recent years, from 600-750 diagnoses in the period 2000-2010 to over 800 diagnoses in 2018-2019. The yearly number of women who die due to cervical cancer has been stable since 2002 at 200 [50]. For the other cancer sites there is no screening program. Over the period 2008-2017, the total estimated yearly number of cancer diagnoses attributable to HPV in the Netherlands was above 1,200 and in 2017-2018 the estimated yearly number of AGW diagnoses was above 43,000 [51, 52]. 


\section{HPV vaccines}

To prevent HPV infection and subsequently HPV-associated diseases, prophylactic vaccines that target HPV have been developed. To date, 3 vaccines have been licensed in Europe; a bivalent (2vHPV) vaccine Cervarix $^{\circledR}$, a quadrivalent (4vHPV) vaccine Gardasil ${ }^{\circledR}$, and a nonavalent (9vHPV) vaccine Gardasil9 ${ }^{\circledR}$ [53-55] (Table 1.2).

Table 1.2. Overview of the 3 currently available prophylactic HPV vaccines.

\begin{tabular}{|c|c|c|c|}
\hline & $\begin{array}{l}\text { Bivalent HPV vaccine } \\
\text { (Cervarix }^{\circledR} \text { ) [53] }\end{array}$ & $\begin{array}{l}\text { Quadrivalent HPV vaccine } \\
\left(\text { Gardasil }^{\circledR}\right) \text { [54] }\end{array}$ & $\begin{array}{l}\text { Nonavalent HPV vaccine } \\
\left(\text { Gardasil9 }{ }^{\circledR}\right)[55]\end{array}$ \\
\hline \multirow{10}{*}{$\begin{array}{l}\text { Composition } \\
\text { per } 1 \text { dose } \\
(0.5 \mathrm{ml})\end{array}$} & $\begin{array}{l}\text { Virus-like particle of L1 } \\
\text { protein of: }\end{array}$ & $\begin{array}{l}\text { Virus-like particle of L1 } \\
\text { protein of: }\end{array}$ & $\begin{array}{l}\text { Virus-like particle of L1 } \\
\text { protein of: }\end{array}$ \\
\hline & HPV16 & HPV6 & $30 \mu \mathrm{g}$ \\
\hline & HPV18 & HPV11 & HPV11 \\
\hline & & HPV16 & HPV16 \\
\hline & & HPV18 & HPV18 \\
\hline & & & HPV31 \\
\hline & & & HPV33 \\
\hline & & & HPV45 \\
\hline & & & HPV52 \\
\hline & & & HPV58 \\
\hline Adjuvant & 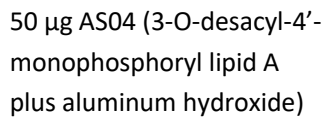 & $\begin{array}{l}225 \mu \mathrm{g} \text { AAHS (amorphous } \\
\text { aluminum } \\
\text { hydroxyphosphate sulfate) }\end{array}$ & $\begin{array}{l}500 \mu \mathrm{g} \text { AAHS (amorphous } \\
\text { aluminum } \\
\text { hydroxyphosphate sulfate) }\end{array}$ \\
\hline \multirow{5}{*}{$\begin{array}{l}\text { Target } \\
\text { population } \\
\text { Vaccination } \\
\text { scheme }\end{array}$} & $\begin{array}{l}\text { Males and females } \geq 9 \text { years } \\
\text { of age }\end{array}$ & $\begin{array}{l}\text { Males and females } \geq 9 \text { years } \\
\text { of age }\end{array}$ & $\begin{array}{l}\text { Males and females } \geq 9 \text { years } \\
\text { of age }\end{array}$ \\
\hline & Intramuscular & Intramuscular & Intramuscular \\
\hline & $\begin{array}{l}\text { 9- to } 14 \text {-year-olds: } 2 \text { doses, } \\
\text { at } 0,6 \text { months }^{\text {a }}\end{array}$ & $\begin{array}{l}\text { 9- to } 13 \text {-year-olds: } 2 \text { doses, } \\
\text { at } 0,6 \text { months }\end{array}$ & $\begin{array}{l}\text { 9- to } 14 \text {-year-olds: } 2 \text { doses, } \\
\text { at } 0,6-12 \text { months }^{d}\end{array}$ \\
\hline & $\geq 15$-year-olds: 3 doses, at & $\geq 14$-year-olds: 3 doses, at & $\geq 15$-year-olds: 3 doses, at \\
\hline & $0,1,6$ months $^{b}$ & $0,2,6$ months $^{c}$ & $0,2,6$ months $^{\mathrm{e}}$ \\
\hline Indications & $\begin{array}{l}\text { Protection against HPV- } \\
\text { related: } \\
\text { - Precancerous cervical, } \\
\text { vulvar, vaginal, and anal } \\
\text { lesions; } \\
\text { - Cervical and anal cancers }\end{array}$ & $\begin{array}{l}\text { Protection against HPV- } \\
\text { related: } \\
\text { - Precancerous cervical, } \\
\text { vulvar, vaginal, and anal } \\
\text { lesions; } \\
\text { - Cervical and anal cancers; } \\
\text { - Genital warts }\end{array}$ & $\begin{array}{l}\text { Protection against HPV- } \\
\text { related: } \\
\text { - Precancerous cervical, } \\
\text { vulvar, vaginal, and anal } \\
\text { lesions; } \\
\text { - Cervical, vulvar, vaginal, } \\
\text { and anal cancers; } \\
\text { - Genital warts }\end{array}$ \\
\hline Notes & $\begin{array}{l}\text { a. } 2^{\text {nd }} \text { dose can be given } \\
\text { between } 5-13 \text { months. } \\
\text { b. } 2^{\text {nd }} \text { dose can be given } \\
\text { between } 1-2.5 \text { months after } 1^{\text {st }} \\
\text { dose. } 3^{\text {th }} \text { dose can be given } \\
\text { between } 5-12 \text { months after } 1^{\text {st }} \\
\text { dose. }\end{array}$ & $\begin{array}{l}\text { c. } 2^{\text {nd }} \text { dose should be given }>1 \\
\text { month after } 1^{\text {st }} \text { dose. } 3^{\text {th }} \text { dose } \\
\text { should be given }>3 \text { months after } \\
\text { the } 2^{\text {nd }} \text { dose and }<12 \text { months } \\
\text { after } 1^{\text {st }} \text { dose. }\end{array}$ & $\begin{array}{l}\text { d. } 2^{\text {nd }} \text { dose can be given between } \\
5-13 \text { months. } \\
\text { e. } 2^{\text {nd }} \text { dose should be given }>1 \\
\text { month after } 1^{\text {st }} \text { dose. } 3^{\text {th }} \text { dose } \\
\text { should be given }>3 \text { months after } \\
\text { the } 2^{\text {nd }} \text { dose and }<12 \text { months } \\
\text { after } 1^{\text {st }} \text { dose. }\end{array}$ \\
\hline
\end{tabular}


All 3 vaccines contain virus-like particles (VLPs) of the major capsid L1 protein of several HPV types. Since VLPs contain no viral DNA the vaccines cannot infect cells, reproduce, or cause disease. The number of HPV types that are targeted, differs per vaccine; all 3 vaccines contain VLPs of the most oncogenic types 16 and 18; the 4VHPV and 9vHPV vaccine also contain VLPs of the IrHPV types 6 and 11; the 9vHPV vaccine additionally contains VLPs of the hrHPV types $31,33,45,52$, and 58 . The 3 vaccines are licensed for males and females from the age of 9 years and indicated to prevent anogenital (pre)cancers caused by vaccine types. The 4vHPV and 9vHPV vaccine are also indicated to prevent AGWs. For 9- to 13/14year-olds, vaccination is given in a 2-dose schedule, while 3 doses of vaccine are required for those who are older at the moment of vaccination. All 3 vaccines have acceptable safety profiles [53-57].

Generally the efficacy of vaccines is measured in double blinded randomized controlled trials (RCTs), where participants are randomly allocated to receive the vaccine under study (intervention arm) or a placebo (control arm). In a trial setting with successful randomization, there will be no differences between the intervention and control arm that could confound the effects of vaccination. Moreover blinding to the vaccine will neutralize possible placebo effects. In an RCT it is therefore assumed that differences in disease occurrence between arms can only be attributed to the effect of the vaccine of interest. RCTs often use different analyses to evaluate the vaccine efficacy; intention-to-treat (ITT), per-protocol (PP), or a variant of these two. ITT analysis generally means evaluation of all participants according to the allocated arm, independent of the actual (number of) vaccine doses they received. PP analysis generally means evaluation of the participants without protocol violations, usually only including those who actually received the vaccine they were allocated to with the correct dosing schedule [58]. For HPV, the distinction between ITT and PP was also based on the HPV DNA and HPV antibody status at the moment of vaccination. The ITT cohorts included those with and without evidence of prior HPV exposure, while the PP cohorts were restricted those HPV DNA negative and HPV seronegative (as a proxy for absence of prior exposure).

RCTs to study the HPV vaccine efficacy have mainly focused on 15- to 26-year-old women using a 3-dose schedule and on protection against cervical HPV. The RCTs were not conducted in the eventual target population of (pre)adolescents because of practical reasons. Trials in this age group would have required follow-up for a really long time, due to the age of sexual activity and the long time to develop clinical disease after acquiring an infection. Because cancer development can take decades, the HPV vaccine efficacy was measured against HPV (persistent) infections and precancerous lesions as surrogate endpoints for cancer. This is also the reason why the vaccines are not indicated to prevent oropharyngeal cancer; due to the absence of well-defined precancerous lesions for 
oropharyngeal cancer, studying the vaccine efficacy against this disease is very timeconsuming. For the 4vHPV vaccine the efficacy was also measured against AGWs. In the evaluation of vaccine efficacy, several subpopulations have been made according to the HPV DNA and HPV antibody status at the moment of vaccination. The population being HPV DNA negative and HPV seronegative at the moment of vaccination was assumed to be the best approximation for the target population of (pre)adolescents before sexual debut [59]. However, it is important to note that HPV DNA and serologic measures will underestimate the true level of (prior) HPV exposure, as people could have a latent infection, not all people seroconvert after an infection, and antibodies may wane over time [60,61].

Most RCTs and with the longest follow-up were those with the 2vHPV and 4vHPV vaccine and most vaccine efficacy measures come from these trials. The trails have shown high vaccine efficacy against persistent infections, AGWs in the case of the 4vHPV vaccine, and precancerous cervical lesions with the HPV vaccine types, especially in those who were HPV DNA negative and HPV seronegative (generally well over 90\%) [56, 62]. The trials showed that vaccination is not effective against HPV infections prevalent at the time of vaccination, meaning that vaccination does not speed up clearance. However among women with HPV DNA of 1 vaccine type present, the vaccine was still effective in preventing infections with the other type(s) [63]. The vaccine efficacy among women who were HPV seropositive when vaccinated was less but still substantial ( $>66 \%$ against persistent cervical infection with the vaccine types) $[64,65]$. These results demonstrate the prophylactic nature of the vaccines and the importance of vaccination before HPV exposure. Although the vaccines do not have a therapeutic effect on prevalent infections, vaccination might still prevent future infections but this is not well studied. Next to the high vaccine efficacy against the vaccine types, the RCTs of the $2 \mathrm{vHPV}$ and the 4VHPV vaccines also hinted towards cross-protection against non-vaccine types, with generally higher and broader signs of cross-protection for the $2 \mathrm{vHPV}$ compared to the 4vHPV vaccine. However, the results of type-specific crossprotection were less conclusive and dependent on the population and outcome studied. Moreover, the duration of the cross-protection has been questioned [66].

There are less RCTs that studied the vaccine efficacy for other anatomical sites. In studies of the 4vHPV vaccine, vaccine efficacy was high against low-grade vulvar and vaginal lesions among women without evidence of prior exposure (>90\%) [67]. In post-hoc analyses of a 2 vHPV vaccine trial, vaccine efficacy was estimated against one-time detection of vulvar, anal, and oral HPV infection among women, which were comparable to estimates against cervical infection [68-70].

There are also much less RCTs among males [71]. In 2 trials, both using the 4vHPV vaccine, the vaccine efficacy has been studied among 16- to 26-year-old men. Results of these trials were comparable to the results among women, with high vaccine efficacy 
against HPV vaccine-type related penile and anal persistent infections and external penile and anal lesions, among those who were HPV DNA negative and seronegative (vaccine efficacy ranging between $78 \%$ and $100 \%$ ) [72-74].

The RCTs have shown that all 3 HPV vaccines induce high antibody responses against the vaccine types, demonstrating that the vaccines are highly immunogenic [59, 75]. Immunobridging studies were used to extrapolate the vaccine efficacy as measured in the RCTs to other target populations or vaccine schedules. Assuming the antibody concentration is an indicator for protection, the rationale behind immunobridging is that an equally high antibody concentration means an equally high vaccine efficacy. For example, if the antibody concentration after vaccination in (pre)adolescent females is non-inferior to the antibody concentration in 15 - to 26 -year-old women among whom vaccine efficacy had been demonstrated, comparable vaccine efficacy is inferred. Using this principle, vaccine efficacy was extrapolated to younger target populations [76-78], males [77-81], and a 2dose schedule [81-83]. Immunobridging was also used to infer the vaccine efficacy of the 9vHPV vaccine against the 4vHPV types 6, 11, 16, and 18 [75].

\section{HPV vaccination program in the Netherlands}

In the Netherlands, free of charge 2vHPV vaccination was introduced in 2009 for girls only, with the main aim to prevent cervical cancer [84]. It started in 2009 with a catch-up program for 12- to 16-year-old girls born in 1993-1996. From 2010 onwards, HPV vaccination was offered to girls in the year they turn 13 years old $[85,86]$. At first, vaccination was given in a 3-dose schedule (given at 0, 1, 6 months). In 2014, the vaccination schedule was changed to a 2-dose schedule (given at 0, 6 months) for girls under the age of 15 years, based on the results of immunobridging studies showing that that a 2-dose schedule given before 15 years of age was as immunogenic as a 3-dose schedule among 15- to 26-year-old women [76]. For girls older than 15, the 3-dose schedule still applied [87]. The vaccination coverage of complete dosing varied between $45.5 \%$ and $61 \%$ depending on the birth cohort (Figure 1.2) $[88,89]$. Preliminary data from January 2020 showed an increased vaccination coverage of the first dose of $72 \%$ among girls born in 2006, who were offered vaccination in 2019 [90]. 


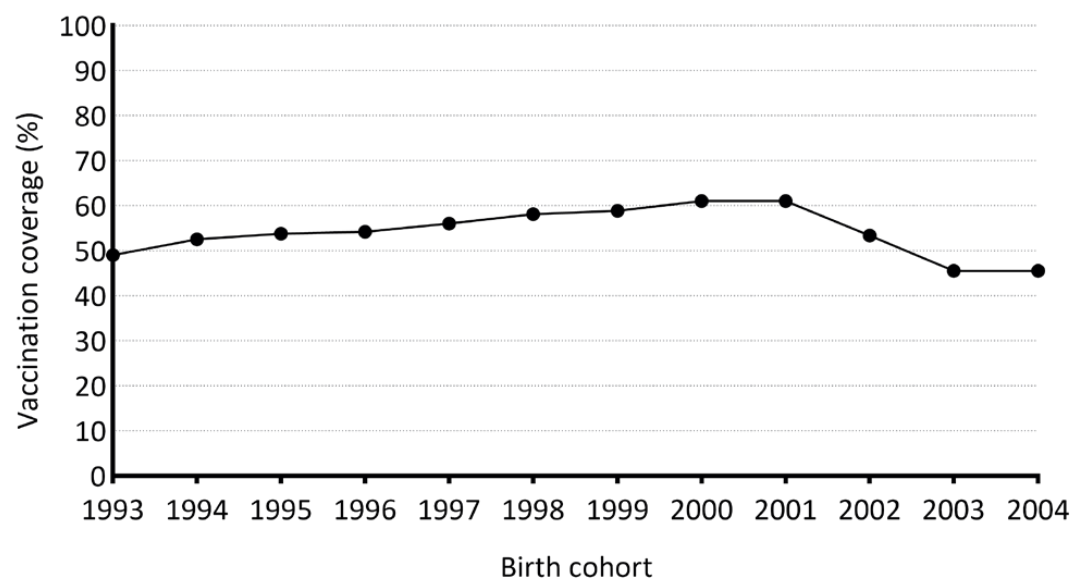

Figure 1.2. HPV vaccination coverage in the Netherlands by birth cohort.

Note: Preliminary data showed an increased vaccination coverage of the first dose of HPV vaccine among girls born in 2005 (59\%) and 2006 (72\%) [90]. Source: Immunization coverage and annual reports national immunization program in the Netherlands [88, 89].

HPV vaccine safety has been studied extensively worldwide and in the Netherlands. Based on these studies HPV vaccines are considered safe [91, 92]. Local reactions (like pain and redness at the injection site), acute reactions (like fainting), and transient systemic reactions (like headaches and muscle strain) are frequently reported among HPV vaccinated individuals. However, based on many studies, including those of the Netherlands, a statistically significant relation between HPV vaccination and severe side effects (like chronic fatigue or auto-immune diseases) have not been found [85, 93].

\section{Measuring vaccine effects}

After implementation of vaccines in national immunization programs, observational studies are required to assess the (long-term) effects of vaccination on the occurrence of the infection in a population at large. Because vaccination will lower the force of infection (rate at which susceptible individuals acquire an infection), the evaluation of the effects of vaccination needs to take into account some methodological challenges [94]. To measure different vaccine effects that can be distinguished, we will follow the theoretical framework as proposed Halloran et al. [94] and adapted by Hanquet et al. [95] and focus on the direct vaccine effectiveness and impact of vaccination (Figure 1.3). Direct vaccine effectiveness is related to the direct protection of the vaccine itself (individual-level effect), while the impact is related to the effects of a vaccination program in the total population (populationlevel effects) [95]. A more detailed description of the direct vaccine effectiveness and impact of vaccination and how they can be measured is provided below. 


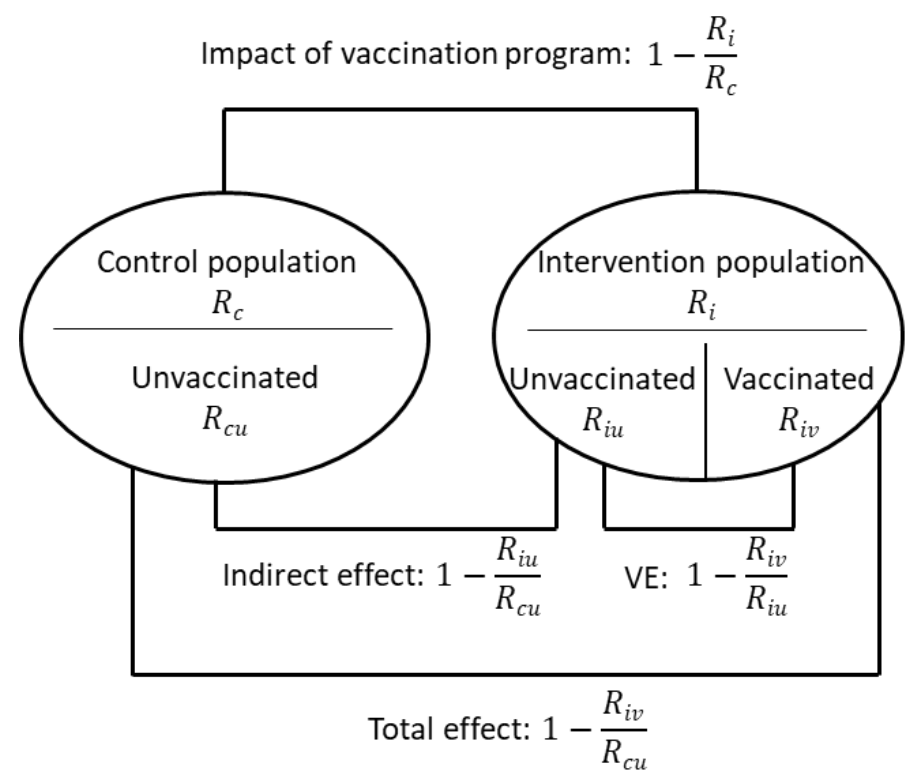

Figure 1.3. Theoretical framework to distinguish and measure different type of vaccine effects, adapted from Halloran et al. [94] and Hanquet et al. [95].

Note: The control population constitutes a population without an established vaccination program, while the intervention population constitutes a population with an established vaccination program. Abbreviation: VE= direct vaccine effectiveness. $\mathrm{Rc}=\mathrm{Rcu}=$ measure of infection risk in the control population (all unvaccinated). $\mathrm{Ri}=$ measure of infection risk in the intervention population (mix between vaccinated and unvaccinated individuals). Riu= measure of infection risk among those unvaccinated in the intervention population. Riv= measure of infection risk among those vaccinated in the intervention population.

\section{Direct vaccine effectiveness}

The direct vaccine effectiveness is also referred to as the direct effect of vaccination under field conditions or field efficacy. We will refer to the direct vaccine effectiveness as VE. It reflects the protection of individuals vaccinated in routine practice $[95,96]$. VE differs from vaccine efficacy in the sense that it is measured under field conditions while vaccine efficacy is measured in an RCT under controlled conditions and in a strict study population [96]. In real-life settings, the cold chain, vaccine storage, and vaccination schedules can be suboptimal. These conditions could possibly affect the VE, making the evaluation of VE in post-implementation studies important. In the case of HPV, the VE can also deviate from the vaccine efficacy, since the RCTs that measured vaccine efficacy included a different study population (15- to 26-year-old women) than the target population of HPV vaccination programs (preadolescent girls) [59]. Because vaccine efficacy was not directly measured in the target population, the evaluation of the HPV VE is even more important.

The VE can be measured by comparing infections among vaccinated individuals with infections among unvaccinated individuals, in a population with an established vaccination program (intervention population in Figure 1.3). Usually, it is estimated as 1 minus some 
measure of risk of infection in vaccinated relative to unvaccinated individuals (i.e. VE=1relative risk). Depending on the study design, this relative risk can be for instance a risk ratio, odds ratio, or prevalence ratio $[95,97]$. In the evaluation of VE, both vaccinated and unvaccinated individuals should have an equal exposure to the infection and equal susceptibility independent of vaccination. Therefore it is important that both vaccinated and unvaccinated individuals belong to the same source population and are exposed to the same vaccination program [95]. Still, in observational studies these assumptions are frequently violated. For example, in studies conducted in the Netherlands there are differences with respect to demographics and risk behavior between women who are vaccinated against HPV and women who are not, either because these differences exist in the general population or because of selective participation in studies [98-100]. These differences could affect the susceptibility or risk of exposure and should therefore be taken into account in the VE estimation, either by controlling for it during the design or analyses phase or during the interpretation of the results [101].

\section{Impact of vaccination}

The impact of a vaccination program is also referred to as the overall effect of vaccination in the entire population or population vaccine effectiveness [95, 102]. It reflects the difference in infection risk between a population with an established vaccination program and the infection risk in the same population without a vaccination program, i.e. the counterfactual. The impact captures the changes in the transmission dynamics in a population due to vaccination, which is usually not the case in traditional pre-licensure RCTs that are designed to measure the vaccine efficacy only [101]. Therefore, postimplementation observational studies are important and needed to measure the eventual population-level effects of a vaccination program. The impact of a vaccination program consists of the weighted sum of 2 more types of vaccine effects, namely the indirect effects of vaccination on unvaccinated individuals and the total effects of vaccination on vaccinated individuals [95].

Indirect effects of vaccination result from reduced circulation of the vaccine-targeted infection, thereby reducing the risk of infection in the entire population including unvaccinated individuals. This indirect effect is called herd protection [103]. In case of sexually transmitted HPV, the sexual partners of vaccinated individuals will also benefit from vaccination. Because the majority of the people identify themselves as heterosexual $[104,105]$, the benefits from vaccination could extend to both sexes even in a girls-only vaccination program [106]. The indirect effects can be measured by comparing infections in unvaccinated individuals in a population with an established vaccination program, with infections in unvaccinated individuals in a control population without a vaccination program as a proxy of the counterfactual (Figure 1.3) [95, 97]. 
The total effects of vaccination for vaccinated individuals include both direct and indirect effects. It reflects the combined benefits of being vaccinated (lower probability of infection given exposure) and being in a population with a vaccination program (lower exposure). The total effects can be measured by comparing infections in vaccinated individuals in a population with a vaccination program with infections in unvaccinated individuals in a control population (Figure 1.3) [95, 97].

The need for an intervention population with a vaccination program and a control population without a vaccination program is methodologically challenging. This is because the populations should be comparable to each other with respect to baseline force of infection and population characteristics, but separated to make transmission between these populations impossible. To overcome the latter, in many studies the control population constitutes a population before a vaccination program has been established and the intervention population constitutes a similar population after a vaccination program has been established. A major assumption in using such a design is that the pre-vaccination population is a valid post-vaccination counterfactual condition and that all relevant factors, other than the vaccination program, remain constant over time. This assumption is violated for example if there are secular trends in the target infection or if risk factors change over time. Another design to measure the impact of vaccination is a cluster RCT, in which clusters or communities are randomized to a vaccination program or not. A major assumption of this design is that the transmission between clusters is not possible, which is not the case when individuals migrate from a control cluster to an intervention cluster or conversely [95, 97]. Moreover, the intervention and control clusters need to be comparable with respect to baseline force of infection and population characteristics, which is often difficult to achieve by randomization because of the limited number of clusters that are usually included in such trials.

The magnitude of the impact of a vaccination program is influenced by a lot of factors. Among the most important are the VE, transmissibility of the infection, mixing patterns (sexual mixing in the case of HPV), vaccination coverage, and the distribution of the vaccination coverage in the population according to risk factors [103]. The transmission potential of an infection can be expressed by the basic reproduction number R0, which is the average number of secondary cases caused by an infected individual if all contacts are with susceptibles. Simply said, the higher the RO the higher the vaccination coverage needs to be to eliminate the infection, classically quantified as $1-1 /$ R0. However, this simple threshold theory assumes random vaccine uptake and homogenous mixing, which is often not the case, and need not apply in sex-specific immunization for STIs [107]. The vaccination coverage among risk groups and mixing patterns within and between risk groups all influence the impact of vaccination [108]. For example for HPV, assortative sexual mixing 
among individuals with high sexual risk behavior can lead to maintained transmission of HPV and lower herd effects. In these populations, the vaccination coverage will need to be higher to realize a large impact of vaccination [108-110].

Not all effects of vaccination programs are favorable. For multi-type pathogens like HPV, types not targeted by the vaccine could take over the ecological nice left vacant by elimination of the vaccine-targeted types. This phenomenon is called type-replacement. For type-replacement to occur, there needs to be some sort of competition between types during natural infection or transmission, so that a decreasing prevalence of a vaccine type after vaccination implementation can lead to an increasing prevalence of a competitive nonvaccine type $[111,112]$. For HPV, type-replacement could play a role, as there are many different HPV types and the available vaccines only target a subset. While some believe the risk of HPV type-replacement is low, it cannot be excluded [113-115]. Possible typereplacement is part of the overall impact of a vaccination program. It can be measured using the above described methods comparing the risk of infection between an intervention and control population, focusing on non-vaccine types.

\section{Aim and outline of this thesis}

The aim of this thesis is to measure the individual- and population-level effects of 2vHPV vaccination. More specifically the aim is to measure the VE of 2VHPV vaccination against HPV positivity and AGWs and to measure the impact of the girls-only 2vHPV vaccination program on the HPV prevalence in the Netherlands.

To measure the individual- and population-level effects of $2 \mathrm{VHPV}$ vaccination on HPV, the PASSYON (PApillomavirus Surveillance among STI clinic YOungsters in the Netherlands) study was set-up in 2009 [116, 117]. In this repeated cross-sectional study, 16- to 24-yearold men and women visiting sexual health centers ( $\mathrm{SHCs}$ ) throughout the Netherlands were tested for type-specific HPV positivity. SHCs (previously called STI clinics) in the Netherlands provide free STI testing and care for people at high risk for STIs, including youth up to 24 years of age [118]. As a consequence, people visiting SHCs are generally at higher risk for HPV infections compared to the general population [119]. The PASSYON study started in 2009 and was repeated every other year using the same study protocol. The first study round in 2009 was before HPV vaccination was implemented in the Netherlands and can be seen as the control population in Figure 1.3. Each subsequent study round was in the postvaccination era and can be seen as an intervention population in Figure 1.3, with a part of the population vaccinated and a part unvaccinated. Using the PASSYON study data and above mentioned theoretical framework, different effects of vaccination can be measured. 
The first part of this thesis focusses on the VE of 2VHPV vaccination against HPV positivity among women who had been eligible for HPV vaccination in the Netherlands, i.e. women born in 1993 or later. We focus on the VE against the vaccine types HPV16 and HPV18 and on the VE against non-vaccine types to study possible cross-protection in the target population. In Chapter 2 the VE against genital type-specific hrHPV positivity is provided using data up to 6 years post-vaccination. In Chapter 3 , we update the measures of VE against genital HPV using data up to 8 years post-vaccination and including IrHPV types. Moreover, to substantiate cross-protection reported across the RCTs, the VE against nonvaccine HPV types is related to the phylogenetic distance of these types towards the vaccine types. Chapter 4 assesses the VE against type-specific anal HPV positivity among women.

The second part of this thesis focusses on the impact of the HPV vaccination program in the Netherlands on the HPV prevalence in the population. The impact of a vaccination program cannot be captured in an RCT, highlighting the importance of post-implementation observational studies. In Chapter 5 trends in the HPV16 and HPV18 prevalence among female and heterosexual male visitors of SHCs are provided from pre-vaccination up to 6 years post-vaccination with the main aim to study herd effects. In Chapter 6 , the aim is to study herd effects for vaccine and cross-protective HPV types and possible typereplacement. We provide trends in the type-specific HPV prevalence for hrHPV as well as IrHPV types from pre-vaccination up to 8 years post-vaccination among women and heterosexual men. In Chapter 7, we studied the impact of the girls-only vaccination program on the HPV prevalence among men who have sex with men (MSM) up to 8 years postvaccination. Moreover to study prior HPV exposure, we assessed the proportion of MSM who were HPV DNA and HPV seronegative when visiting the SHC, which informs the opportunities for targeted vaccination in this high-risk group.

Although the 2vHPV vaccine is not indicated to prevent HPV6 or HPV11 infections, there are indications the $2 \mathrm{vHPV}$ vaccine might provide protection against AGWs. However results regarding this protection have been inconclusive. The third part of this theses focusses on the VE of $2 \mathrm{VHPV}$ vaccination against AGWs among women who had been eligible for HPV vaccination in the Netherlands. In Chapter 8 , we calculate the effect of HPV vaccination on the occurrence of AGWs diagnosed at the SHC using data from the PASSYON study. In this chapter, we also calculate the effect of HPV vaccination on the most important types causing AGWs, HPV6/11 positivity. Because most AGWs are diagnoses by general practitioners, in Chapter 9 we assess the VE of 2 VHPV vaccination against AGWs, using data from general practitioners linked to the vaccination registry.

Last, in Chapter 10 the main findings of this thesis are summarized and discussed. Future perspectives are given for measuring the effects of HPV vaccination. 


\section{References}

1. Harden ME, et al. Human papillomavirus molecular biology. Mutat Res Rev Mutat Res 2017; 772:3-12.

2. Bzhalava $D$, et al. International standardization and classification of human papillomavirus types. Virology 2015; 476:341-4.

3. de Villiers EM, et al. Classification of papillomaviruses. Virology 2004; 324:17-27.

4. Bernard $\mathrm{HU}$, et al. Classification of papillomaviruses (PVs) based on 189 PV types and proposal of taxonomic amendments. Virology 2010; 401:70-9.

5. Doorbar J, et al. The biology and life-cycle of human papillomaviruses. Vaccine 2012; 30 Suppl 5:F55-70.

6 . Bouvard V, et al. A review of human carcinogens-Part B: biological agents. Lancet Oncol 2009; 10:3212.

7. de Sanjose S, et al. The natural history of human papillomavirus infection. Best Pract Res Clin Obstet Gynaecol 2018; 47:2-13.

8. Gupta SM, et al. Molecular mechanisms in progression of HPV-associated cervical carcinogenesis. J Biomed Sci 2019; 26:28.

9. Wakabayashi R, et al. The Host-Microbe Interplay in Human Papillomavirus-Induced Carcinogenesis. Microorganisms 2019; 7.

10. Rodriguez AC, et al. Longitudinal study of human papillomavirus persistence and cervical intraepithelial neoplasia grade 2/3: critical role of duration of infection. J Natl Cancer Inst 2010; 102:315-24.

11. Winer RL, et al. Early natural history of incident, type-specific human papillomavirus infections in newly sexually active young women. Cancer Epidemiol Biomarkers Prev 2011; 20:699-707.

12. Taylor $\mathrm{S}$, et al. The incidence, clearance and persistence of non-cervical human papillomavirus infections: a systematic review of the literature. BMC Infect Dis 2016; 16:293.

13. Giuliano $A R$, et al. Incidence and clearance of genital human papillomavirus infection in men (HIM): a cohort study. Lancet 2011; 377:932-40.

14. Marra E, et al. Incidence and Clearance of Anal High-risk Human Papillomavirus Infections and Their
Determinants Over 5 Years Among Human Immunodeficiency Virus-negative Men Who Have Sex With Men. Clin Infect Dis 2019; 68:1556-65.

15. Gravitt PE, et al. Natural History of HPV Infection across the Lifespan: Role of Viral Latency. Viruses 2017; 9 .

16. Twisk DE, et al. Detection of Incident Anal HighRisk Human Papillomavirus DNA in Men Who Have Sex With Men: Incidence or Reactivation? J Infect Dis 2018; 218:1018-26.

17. Burger EA, et al. Estimating the Natural History of Cervical Carcinogenesis Using Simulation Models: A CISNET Comparative Analysis. J Natl Cancer Inst 2019.

18. Vink MA, et al. Clinical progression of high-grade cervical intraepithelial neoplasia: estimating the time to preclinical cervical cancer from doubly censored national registry data. Am J Epidemiol 2013; 178:1161-9.

19. Zhang J, et al. Spontaneous Regression of Cervical Intraepithelial Neoplasia 2: A Meta-analysis. Gynecol Obstet Invest 2019; 84:562-7.

20. Ostor AG. Natural history of cervical intraepithelial neoplasia: a critical review. Int J Gynecol Pathol 1993; 12:186-92.

21. McCredie MRE, et al. Natural history of cervical neoplasia and risk of invasive cancer in women with cervical intraepithelial neoplasia 3 : a retrospective cohort study. The Lancet Oncology 2008; 9:425-34.

22. Siddharthan RV, et al. Anal intraepithelial neoplasia: diagnosis, screening, and treatment. Ann Gastroenterol 2019; 32:257-63.

23. Venkatesan NN, et al. Recurrent respiratory papillomatosis. Otolaryngol Clin North Am 2012; 45:671-94, viii-ix.

24. Garland SM, et al. Natural history of genital warts: analysis of the placebo arm of 2 randomized phase III trials of a quadrivalent human papillomavirus (types 6, 11, 16, and 18) vaccine. J Infect Dis 2009; 199:805-14.

25. Park IU, et al. Human Papillomavirus and Genital Warts: A Review of the Evidence for the 2015 Centers for Disease Control and Prevention Sexually Transmitted Diseases Treatment Guidelines. Clin Infect Dis 2015; 61 Suppl 8:S849-55. 
26. Chesson HW, et al. The estimated lifetime probability of acquiring human papillomavirus in the United States. Sex Transm Dis 2014; 41:660-4.

27. Veldhuijzen NJ, et al. Factors affecting transmission of mucosal human papillomavirus. The Lancet Infectious Diseases 2010; 10:862-74.

28. Lam JU, et al. Condom use in prevention of Human Papillomavirus infections and cervical neoplasia: systematic review of longitudinal studies. J Med Screen 2014; 21:38-50.

29. Poljak $M$, et al. Anogenital hairs are an important reservoir of alpha-papillomaviruses in patients with genital warts. J Infect Dis 2009; 199:1270-4.

30. Rodriguez AC, et al. The natural history of human papillomavirus infection and cervical intraepithelial neoplasia among young women in the Guanacaste cohort shortly after initiation of sexual life. Sex Transm Dis 2007; 34:494-502.

31. Zou $\mathrm{H}$, et al. Early acquisition of anogenital human papillomavirus among teenage men who have sex with men. J Infect Dis 2014; 209:642-51.

32. Coupe VM, et al. Age-dependent prevalence of 14 high-risk HPV types in the Netherlands: implications for prophylactic vaccination and screening. Br J Cancer 2008; 98:646-51.

33. Anic GM, et al. Genital HPV infection and related lesions in men. Prev Med 2011; 53 Suppl 1:S36-41.

34. Poynten IM, et al. Comparison of age-specific patterns of sexual behaviour and anal HPV prevalence in homosexual men with patterns in women. Sex Transm Infect 2016; 92:228-31.

35. Chaturvedi AK. Beyond cervical cancer: burden of other HPV-related cancers among men and women. J Adolesc Health 2010; 46:S20-6.

36. Walboomers JM, et al. Human papillomavirus is a necessary cause of invasive cervical cancer worldwide. J Pathol 1999; 189:12-9.

37. de Martel C, et al. Worldwide burden of cancer attributable to HPV by site, country and HPV type. Int J Cancer 2017; 141:664-70.

38. van Alewijk $D$, et al. A human papilloma virus testing algorithm comprising a combination of the $\mathrm{L} 1$ broad-spectrum SPF10 PCR assay and a novel E6 high-risk multiplex type-specific genotyping PCR assay. J Clin Microbiol 2013; 51:1171-8.

39. de Sanjose $S$, et al. Human papillomavirus genotype attribution in invasive cervical cancer: a retrospective cross-sectional worldwide study. Lancet Oncol 2010; 11:1048-56.

40. de Sanjose $S$, et al. Burden of Human Papillomavirus (HPV)-Related Cancers Attributable to HPVs $6 / 11 / 16 / 18 / 31 / 33 / 45 / 52$ and $58 . \mathrm{JNCl}$ Cancer Spectr 2018; 2:pky045.

41. International Agency for Research on Cancer (IARC). Global Cancer Observatory. Source: Globocan 2018. Available at: http://gco.iarc.fr/. Accessed 0702-2020.

42. Frisch $M$, et al. Cancer in a population-based cohort of men and women in registered homosexual partnerships. Am J Epidemiol 2003; 157:966-72.

43. Chaturvedi AK. Epidemiology and clinical aspects of HPV in head and neck cancers. Head Neck Pathol 2012; 6 Suppl 1:S16-24.

44. Kang YJ, et al. Anal cancer in high-income countries: Increasing burden of disease. PLoS One 2018; 13:e0205105.

45. Kang YJ, et al. Vulvar cancer in high-income countries: Increasing burden of disease. Int J Cancer 2017; 141:2174-86.

46. Hartwig S, et al. Estimation of the overall burden of cancers, precancerous lesions, and genital warts attributable to 9-valent HPV vaccine types in women and men in Europe. Infect Agent Cancer 2017; 12:19. 47. McDonald SA, et al. Disease burden of human papillomavirus infection in the Netherlands, 19892014: the gap between females and males is diminishing. Cancer Causes Control 2017; 28:203-14. 48. De Gier B, et al. [Staat van infectieziekten in Nederland, 2017]. Bilthoven: RIVM, 2018. Nr: 20180032.

49. van der Aa MA, et al. Mass screening programmes and trends in cervical cancer in Finland and the Netherlands. Int J Cancer 2008; 122:1854-8.

50. Netherlands Cancer Registry (NCR). www.cijfersoverkanker.nl. Accessed 24-02-2020.

51. Slurink IAL, et al. Sexually transmitted infections in the Netherlands in 2018. Bitlhoven: RIVM, 2019. Nr: 2019-0007; DOI: 10.21945/RIVM-2019-0007.

52. National Institute for Public Health and the Environment (RIVM). Projected direct benefit of vaccination against HPV-related cancer in the Netherlands Bilthoven: RIVM, 2020.

53. European Medicines Agency (EMA). Cervarix: EPAR - Product Information. Available at: 
https://www.ema.europa.eu/en/medicines/human /EPAR/cervarix. Accessed 17-10-2019.

54. European Medicines Agency (EMA). Gardasil: EPAR - Product Information. Available at: https://www.ema.europa.eu/en/medicines/human /EPAR/gardasil. Accessed 17-10-2019.

55. European Medicines Agency (EMA). Gardasil 9: EPAR - Product Information. Available at: https://www.ema.europa.eu/en/medicines/human /EPAR/gardasil-9. Accessed 17-10-2019.

56. Arbyn M, et al. Efficacy and safety of prophylactic HPV vaccines. A Cochrane review of randomized trials. Expert Rev Vaccines 2018; 17:1085-91.

57. Costa APF, et al. Safety of Human Papillomavirus 9-Valent Vaccine: A Meta-Analysis of Randomized Trials. J Immunol Res 2017; 2017:3736201.

58. Bhide $A$, et al. A simplified guide to randomized controlled trials. Acta Obstet Gynecol Scand 2018; 97:380-7.

59. Schiller JT, et al. A review of clinical trials of human papillomavirus prophylactic vaccines. Vaccine 2012; 30 Suppl 5:F123-38.

60. Mollers $M$, et al. Review: current knowledge on the role of HPV antibodies after natural infection and vaccination: implications for monitoring an HPV vaccination programme. J Med Virol 2013; 85:137985.

61. Giuliano AR, et al. Seroconversion Following Anal and Genital HPV Infection in Men: The HIM Study. Papillomavirus Res 2015; 1:109-15.

62. Harper DM, et al. HPV vaccines - A review of the first decade. Gynecol Oncol 2017; 146:196-204.

63. Future II Study Group. Prophylactic efficacy of a quadrivalent human papillomavirus (HPV) vaccine in women with virological evidence of HPV infection. J Infect Dis 2007; 196:1438-46.

64. Szarewski A, et al. Efficacy of the human papillomavirus (HPV)-16/18 ASO4-adjuvanted vaccine in women aged $15-25$ years with and without serological evidence of previous exposure to HPV16/18. Int J Cancer 2012; 131:106-16.

65. Castellsague $X$, et al. End-of-study safety, immunogenicity, and efficacy of quadrivalent HPV (types $6,11,16,18$ ) recombinant vaccine in adult women 24-45 years of age. Br J Cancer 2011; 105:2837.
66. Malagon T, et al. Cross-protective efficacy of two human papillomavirus vaccines: a systematic review and meta-analysis. Lancet Infect Dis 2012; 12:781-9. 67. Future I/II Study Group. Four year efficacy of prophylactic human papillomavirus quadrivalent vaccine against low grade cervical, vulvar, and vaginal intraepithelial neoplasia and anogenital warts: randomised controlled trial. BMJ 2010; 341:c3493.

68. Kreimer AR, et al. Efficacy of a bivalent HPV 16/18 vaccine against anal HPV 16/18 infection among young women: a nested analysis within the Costa Rica Vaccine Trial. Lancet Oncol 2011; 12:862-70.

69. Herrero R, et al. Reduced prevalence of oral human papillomavirus (HPV) 4 years after bivalent $H P V$ vaccination in a randomized clinical trial in Costa Rica. PLoS One 2013; 8:e68329.

70. Lang Kuhs KA, et al. Reduced prevalence of vulvar HPV16/18 infection among women who received the HPV16/18 bivalent vaccine: a nested analysis within the Costa Rica Vaccine Trial. J Infect Dis 2014; 210:1890-9.

71. Harder T, et al. Efficacy, effectiveness and safety of vaccination against human papillomavirus in males: a systematic review. BMC Med 2018; 16:110. 72. Giuliano AR, et al. Efficacy of quadrivalent HPV vaccine against $H P V$ Infection and disease in males. N Engl J Med 2011; 364:401-11.

73. Palefsky JM, et al. HPV vaccine against anal HPV infection and anal intraepithelial neoplasia. N Engl J Med 2011; 365:1576-85.

74. Mikamo $H$, et al. Efficacy, safety, and immunogenicity of a quadrivalent HPV vaccine in Japanese men: A randomized, Phase 3, placebocontrolled study. Vaccine 2019; 37:1651-8.

75. Huh WK, et al. Final efficacy, immunogenicity, and safety analyses of a nine-valent human papillomavirus vaccine in women aged $16-26$ years: a randomised, double-blind trial. The Lancet 2017; 390:2143-59.

76. Pedersen $\mathrm{C}$, et al. Immunization of early adolescent females with human papillomavirus type 16 and 18 L1 virus-like particle vaccine containing ASO4 adjuvant. J Adolesc Health 2007; 40:564-71.

77. Block SL, et al. Comparison of the immunogenicity and reactogenicity of a prophylactic quadrivalent human papillomavirus (types 6, 11, 16, 
and 18) L1 virus-like particle vaccine in male and female adolescents and young adult women. Pediatrics 2006; 118:2135-45.

78. Van Damme $P$, et al. Immunogenicity and Safety of a 9-Valent HPV Vaccine. Pediatrics 2015; 136:e2839.

79. Petaja $T$, et al. Immunogenicity and safety of human papillomavirus (HPV)-16/18 AS04adjuvanted vaccine in healthy boys aged $10-18$ years. J Adolesc Health 2009; 44:33-40.

80. Castellsague $X$, et al. Immunogenicity and safety of the 9-valent HPV vaccine in men. Vaccine 2015; 33:6892-901.

81. Iversen $\mathrm{OE}$, et al. Immunogenicity of the 9-Valent HPV Vaccine Using 2-Dose Regimens in Girls and Boys vs a 3-Dose Regimen in Women. JAMA 2016; 316:2411-21.

82. Romanowski B, et al. Sustained immunogenicity of the HPV-16/18 AS04-adjuvanted vaccine administered as a two-dose schedule in adolescent girls: Five-year clinical data and modeling predictions from a randomized study. Hum Vaccin Immunother 2016; 12:20-9.

83. Dobson SR, et al. Immunogenicity of 2 doses of HPV vaccine in younger adolescents vs 3 doses in young women: a randomized clinical trial. JAMA 2013; 309:1793-802.

84. Gezondheidsraad. Vaccination against cervical cancer. [Vaccinatie tegen baarmoederhalskanker]. Den Haag: Gezondheidsraad, 2008. Nr: 2008/08.

85. Qendri V, et al. Ten years of HPV vaccination in the Netherlands: current evidence and future challenges in HPV-related disease prevention. Expert Rev Vaccines 2018; 17:1093-104.

86. National Institute for Public Health and the Environment (RIVM). [Richtlijn Uitvoering RVP 2019]. Available at: https://rijksvaccinatieprogramma.nl/professionals/r ichtlijnen/rvp-richtlijn-uitvoering-2019. Accessed 20-11-2019.

87. Donken $\mathrm{R}$, et al. Human papillomavirus (HPV) infection. In: Schurink-van 't Klooster TM, de Melker $\mathrm{HE}$, eds. The National Immunisation Programme in the Netherlands - Surveillance and developments in 2013-2014. Bilthoven: RIVM, 2014:77-85.
88. van Lier EA, et al. [Vaccinatiegraad Rijksvaccinatieprogramma Nederland: Verslagjaar 2011]. Bilthoven: RIVM, 2011. Nr: 210021014/2011. 89. van Lier EA, et al. [Vaccinatiegraad en jaarverslag Rijksvaccinatieprogramma Nederland 2018]. Bilthoven: RIVM, 2019. Nr: 2019-0015.

90. National Institute for Public Health and the Environment (RIVM). [Meer kinderen gevaccineerd tegen bof, mazelen, rodehond en HPV]. Available at: https://www.rivm.nl/nieuws/meer-kinderengevaccineerd-tegen-bof-mazelen-rode-hond-enhpv. Accessed 12-02-2020.

91. IPVS Policy statement on safety of HPV vaccines. Papillomavirus Res 2016; 2:9-10.

92. World Health Organization (WHO). Safety update of HPV vaccines. Available at: https://www.who.int/vaccine_safety/committee/to pics/hpv/June_2017/en/. Accessed 21-11-2019.

93. European Medicines Agency (EMA). HPV vaccines: EMA confirms evidence does not support that they cause CRPS or POTS. London, United Kingdom: European Medicines Agency, 2016. Nr: EMA/788882/2015.

94. Halloran ME, et al. Study designs for dependent happenings. Epidemiology 1991; 2:331-8.

95. Hanquet $\mathrm{G}$, et al. Vaccine effects and impact of vaccination programmes in post-licensure studies. Vaccine 2013; 31:5634-42.

96. Weinberg GA, et al. Vaccine epidemiology: efficacy, effectiveness, and the translational research roadmap. J Infect Dis 2010; 201:1607-10.

97. Halloran ME, et al. Design and Analysis of Vaccine Studies. New York: Springer, 2010 (Gail M, Krickeberg K, Samet J, Tsiatis A, Wong W, eds. Statistics for Biology and Health).

98. Mollers $M$, et al. Effectiveness of human papillomavirus vaccine against incident and persistent infections among young girls: Results from a longitudinal Dutch cohort study. Vaccine 2015; 33:2678-83.

99. Donken R, et al. High Effectiveness of the Bivalent Human Papillomavirus (HPV) Vaccine Against Incident and Persistent HPV Infections up to 6 Years After Vaccination in Young Dutch Women. J Infect Dis 2018; 217:1579-89. 
100. Rondy M, et al. Determinants for HPV vaccine uptake in the Netherlands: A multilevel study. Vaccine 2010; 28:2070-5.

101. Chen RT, et al. Epidemiologic methods in immunization programs. Epidemiol Rev 1996; 18:99-

117.

102. Haber M. Estimation of the population effectiveness of vaccination. Stat Med 1997; 16:60110.

103. Fine $P$, et al. "Herd immunity": a rough guide. Clin Infect Dis 2011; 52:911-6.

104. Woestenberg PJ, et al. Herpes simplex virus type 1 and type 2 in the Netherlands: seroprevalence, risk factors and changes during a 12-year period. BMC Infect Dis 2016; 16:364.

105. de Graaf H, et al. [Seks onder je 25e]. Utrecht: Rutgers, Soa aids Nederland, 2017.

106. Garnett GP. Role of herd immunity in determining the effect of vaccines against sexually transmitted disease. J Infect Dis 2005; 191 Suppl 1:S97-106.

107. Bogaards JA, et al. Sex-specific immunization for sexually transmitted infections such as human papillomavirus: insights from mathematical models. PLoS Med 2011; 8:e1001147.

108. Malagon T, et al. Human papillomavirus vaccination and the role of herd effects in future cancer control planning: a review. Expert Rev Vaccines 2018; 17:395-409.

109. Hughes JP, et al. The theoretical populationlevel impact of a prophylactic human papilloma virus vaccine. Epidemiology 2002; 13:631-9.

110. Malagon T, et al. The impact of differential uptake of HPV vaccine by sexual risks on health inequalities: a model-based analysis. Vaccine 2013; 31:1740-7.

111. Lipsitch M. Vaccination against colonizing bacteria with multiple serotypes. Proc Natl Acad Sci U S A 1997; 94:6571-6.

112. Elbasha $E H$, et al. Vaccination against multiple HPV types. Math Biosci 2005; 197:88-117.

113. Dillner J, et al. Monitoring of human papillomavirus vaccination. Clin Exp Immunol 2011; 163:17-25.

114. Tota JE, et al. Epidemiologic Evaluation of Human Papillomavirus Type Competition and the Potential for Type Replacement Post-Vaccination. PLoS One 2016; 11:e0166329.

115. Safaeian M, et al. Invited commentary: multiple human papillomavirus infections and type replacement-anticipating the future after human papillomavirus vaccination. Am J Epidemiol 2014; 180:1076-81.

116. Vriend $\mathrm{HJ}$, et al. Type-specific human papillomavirus infections among young heterosexual male and female STI clinic attendees. Sex Transm Dis 2012; 39:72-8.

117. Vriend $\mathrm{HJ}$, et al. Patterns of human papillomavirus DNA and antibody positivity in young males and females, suggesting a site-specific natural course of infection. PLoS One 2013; 8:e60696.

118. Visser $M$, et al. Sexually transmitted infections in the Netherlands in 2016. Bitlhoven: RIVM, 2017. Nr: 2017-0003; DOI: 10.21945/RIVM-2017-0003.

119. Mollers $M$, et al. Population- and type-specific clustering of multiple HPV types across diverse risk populations in the Netherlands. Am J Epidemiol 2014; 179:1236-46. 




\section{Part 1}

Direct vaccine effectiveness against HPV positivity 



\section{Chapter 2}

\section{Bivalent vaccine effectiveness against type-specific HPV positivity: Evidence for cross-protection against oncogenic types among Dutch STI clinic visitors}

Petra J. Woestenberg

Audrey J. King

Birgit H. B. van Benthem

Robine Donken

Suzan Leussink

Fiona R. M. van der Klis

Hester E. de Melker

Marianne A. B. van der Sande

Christian J. P. A. Hoebe

Johannes A. Bogaards

The contents of this chapter have been published in The Journal of Infectious Diseases 


\section{Abstract}

Introduction: Observational post-marketing studies are important to assess vaccine effectiveness (VE). We estimated VE from the bivalent human papillomavirus (HPV) vaccine against HPV positivity of vaccine and non-vaccine types in a high-risk population.

Methods: We included all vaccine-eligible women from the PASSYON study, a biennial cross-sectional survey in Dutch sexually transmitted infection clinics. Cervicovaginal swabs were analyzed using a polymerase chain reaction-based assay (SPF10-LiPA25) able to detect the 12 high-risk HPV (hrHPV) types 16/18/31/33/35/39/45/51/52/56/58/59. We compared hrHPV positivity between self-reported vaccinated ( $\geq 1$ dose) and unvaccinated women, and estimated VE by a logistic mixed model.

Results: We included 1,087 women of which 53\% were hrHPV positive and $60 \%$ reported to be vaccinated. The adjusted pooled VE against HPV16/18 was 89.9\% (95\% confidence interval 81.7\%-94.4\%). Moreover, we calculated significant VE against non-vaccine types HPV45 (91\%), HPV35 (57\%), HPV31 (50\%), and HPV52 (37\%). Among women who were offered vaccination 5/6 years ago, we estimated similar VE against HPV16/18 (92\%) and all hrHPV types (35\%) compared to women who were offered vaccination $<5$ years ago ( $83 \%$ and $33 \%$, respectively).

Discussion: We demonstrated high VE of the bivalent vaccine against HPV16/18 and crossprotection against HPV45/35/31/52. Protection against HPV16/18 was sustained up to 6 years post-vaccination. 


\section{Introduction}

Human papillomavirus (HPV) is a sexually transmitted virus that is considered a necessary factor in the development of cervical cancer [1]. Many different HPV types have been identified and classified as high-risk HPV (hrHPV) or low-risk HPV (IrHPV) based on their oncogenic potential [2]. HrHPV types 16 and 18 are associated with approximately $71 \%$ of all cervical cancer cases. Other hrHPV types frequently identified in cervical cancers (together in approximately $21 \%$ of the cancers) are $31,33,35,45,52$, and 58 [3]. Prevention of infection with HPV16/18 and other hrHPV by means of prophylactic vaccination provides a tremendous opportunity to prevent cancer [4].

To date, 3 vaccines have been licensed for the prevention of HPV-related cancer, providing direct protection against 2,4 , or 9 HPV types. The national immunization program (NIP) of the Netherlands uses the bivalent HPV (2vHPV) vaccine Cervarix ${ }^{\circledR}$, which was licensed in 2007 and targets HPV types 16 and 18 [5]. The Dutch HPV vaccination program started in 2009 with a catch-up campaign for girls born in 1993-1996 (12-16 years old). From 2010 onwards, girls are offered vaccination in the year they turn 13 years old, starting with birth cohort 1997 [6].

The $2 \mathrm{vHPV}$ vaccine trials invariably showed high efficacy against persistent HPV16/18 infection and associated precancer lesions of over 90\% [7]. Moreover, some level of crossprotection against non-vaccine hrHPV types was shown in the vaccine trials, but results are less conclusive and dependent on the population and outcome studied [7-10].

Observational studies after the implementation of large-scale immunization programs are important to assess the vaccine effectiveness (VE) against both the vaccine and nonvaccine types in the population at large. Direct effectiveness measures of the $2 \mathrm{vHPV}$ vaccine from observational studies are becoming available in the Netherlands [11, 12], as well as other countries [13-16]. These studies showed high VE from a 3-dose schedule against the vaccine types, ranging between $73 \%$ and $100 \%$ [12-15]. There are also indications for crossprotection of the 2vHPV vaccine from observational studies; in a recently published paper, high VE against HPV31, HPV33, and HPV45 was observed among women attending their first cervical screening in Scotland [15]. However, type-specific estimates of VE against hrHPV types other than HPV16/18/31/33/45 are not yet available in a population-based setting.

Knowledge about the cross-protective VE is important to understand the overall VE and potential clinical impact of the $2 \mathrm{vHPV}$ vaccination program. It is also important for vaccine comparisons in health economic assessments $[17,18]$, especially in view of the more recently licensed nonavalent HPV (9vHPV) vaccine that targets 5 additional hrHPV types associated with about $19 \%$ of all cervical cancer cases (HPV31/33/45/52/58) [19]. Here, we provide direct VE estimates from the $2 \mathrm{VHPV}$ vaccine against hrHPV DNA positivity using 
cross-sectional data from a biennial survey in Dutch sexually transmitted infection (STI) clinics (PASSYON study). We present the VE against type-specific HPV DNA positivity as well as pooled estimates of VE.

\section{Methods}

\section{Study design and population}

The PASSYON (PApillomavirus Surveillance among STI clinic YOungsters in the Netherlands) study is a biennial cross-sectional survey among 16- to 24-year-old STI clinic visitors that started in 2009, when HPV vaccination was implemented in the Netherlands (Figure 2.1). The study design is described in detail elsewhere [20]. Briefly, additional to the routine STI consultation, participants were asked to provide a self-collected genital swab for HPV testing and to fill in a questionnaire including self-reported vaccination status. From participants who provided blood for routine syphilis and HIV testing at the STI clinic, serum was collected for HPV serology. Initially, all people attending the STI clinic provided blood, but due to policy changes from 2013 onwards, only specific groups at high risk for syphilis or HIV provided blood. The Medical Ethical Committee of the University of Utrecht, the Netherlands approved this study (protocol number 08/397). Data were obtained using a unique code per person and all participants gave informed consent.

To calculate the VE, we included from the PASSYON study years 2011-2015 all women who had been eligible for vaccination in the Netherlands (i.e. women born in 1993 or later [6]), who reported their vaccination status, and who provided a cervicovaginal swab.

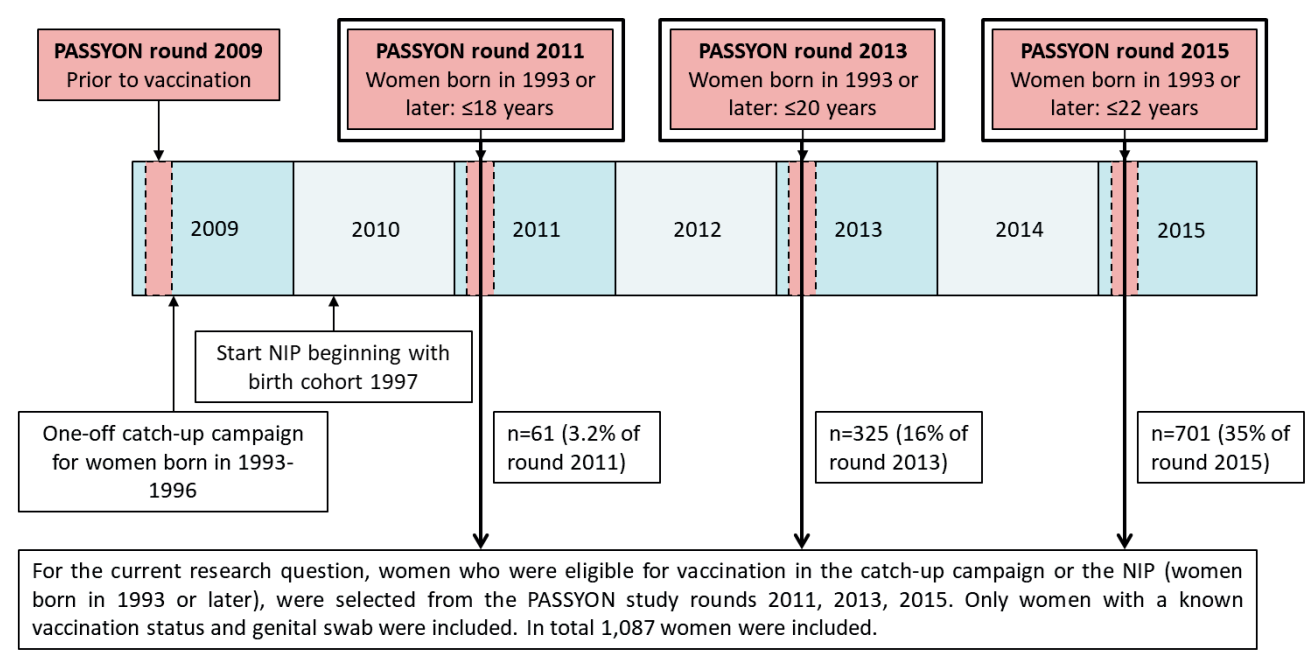

Figure 2.1. HPV vaccination in the Netherlands, the PASSYON study design, and the study population selection. 


\section{Laboratory methods}

Swabs were stored at $-20^{\circ} \mathrm{C}$ until analyses. DNA was extracted using the MagnaPure platform (Total Nucleic Acid Isolation Kit, Roche, the Netherlands) and eluted in 100microliter elution buffer. HPV DNA was amplified using the SPF10 primer set. Subsequently, HPV-specific amplicons were detected using the DNA enzyme linked immunoassay (HPVDEIA, DDL Diagnostics Laboratory, the Netherlands). Amplicons of positive samples were genotyped with the Line probe assay (HPV-LiPA25, DDL Diagnostics Laboratory, the Netherlands), which is able to detect the 12 hrHPV types 16/18/31/33/35/39/45/51/52/56/ 58/59 [20].

Serum samples were stored at $-80^{\circ} \mathrm{C}$ until analyses [21]. HPV antibodies against L1 viruslike particles for types 16 and 18 were assessed using a multiplex immunoassay. Cut-off levels for seropositivity were 9 Luminex Units (LU)/mL for HPV16 and $13 \mathrm{LU} / \mathrm{mL}$ for HPV18 [22].

\section{Validation of self-reported vaccination status}

We used serology to validate the self-reported vaccination status among those who provided blood. We compared the HPV16 and HPV18 seropositivity rates and antibody concentrations between self-reported vaccinated ( $\geq 1$ dose) and unvaccinated women. To check the discriminative ability of antibody concentrations with respect to self-reported vaccination status, we calculated the area under the curve (AUC) of a receiver operating characteristic (ROC).

\section{Statistical analyses}

We checked for differences in potential confounders between vaccinated and unvaccinated women using $\chi^{2}$ tests. We included the demographic variables age, migration background, and education level. Migration background was based on (parental) country of birth. A woman was defined as native Dutch if both parents were born in the Netherlands [23]. Education level was self-reported and categorized as high and low/middle. We also included the number of sex partners in the past 6 months, number of lifetime sex partners, age at sexual debut (defined as vaginal or anal intercourse), history of STIs, condom use with casual partners in the past 6 months, hormonal contraceptives use, and current genital chlamydia or gonorrhea infection. Chlamydia and gonorrhea infection were diagnosed during the routine STI consultation. The other variables were self-reported and categorized (Table 2.1).

Cervicovaginal hrHPV DNA positivity was compared between women who reported to be vaccinated ( $\geq 1$ dose) and women who reported to be unvaccinated. Outcomes were type-specific hrHPV positivity, the vaccine types HPV16/18 (pooled), the hrHPV types included in the 9vHPV vaccine (HPV16/18/31/33/45/52/58, pooled), and all hrHPV types (HPV16/18/31/33/35/39/45/51/52/56/58/59, pooled). We used odds ratios (ORs) to 
estimate the VE, which is suggested to be a suitable measure for the relative reduction in HPV positivity (the combination of incidence and duration of an HPV infection) from crosssectional data [24]. Because we were interested in the VE on an individual level to give the best approximation of the trial efficacy estimates, we calculated the ORs using a logistic mixed model, incorporating all hrHPV types and a random intercept to account for residual dependence between type-specific infections within individuals. This is an efficient method compared to standard logistic regression, because the covariates' coefficients are estimated from all HPV types simultaneously and the measurement of VE against multiple HPV types (pooled outcomes) is specified as a weighted average [25]. All analyses were adjusted for the variables that were associated with vaccination status $(P<.1)$. VE was calculated as 1 minus the adjusted OR times $100 \%$ [26].

Because vaccine efficacy is reduced when recipients are HPV positive at vaccination [5, 27], we calculated the VE against the pooled outcomes separately among women who were (possibly) sexually active when vaccination was offered and among women who were not yet sexually active when vaccination was offered. For the catch-up birth cohorts (19931996), vaccination of the first dose was offered on 1 March 2009 and for the birth cohorts from 1997 onwards, vaccination of the first dose was offered on 1 March in the year they turned 13 years old [28]. We compared the self-reported age of sexual debut with the age when vaccination was offered, and categorized women into either not sexually active if the age when vaccination was offered preceded sexual debut, or (possibly) sexually active otherwise (including women who reported the same age of sexual debut as the age when vaccination was offered). Moreover, as cross-protection has been suggested to wane over time [29], we calculated the VE against the pooled outcomes separately among women who were offered vaccination $<5$ years ago and among women who were offered vaccination $5 / 6$ years ago. This categorization was chosen to have more or less equal numbers in each subgroup. The stratified analyses were adjusted for the variables that were associated with vaccination status $(P<.1)$ as well as the age at which the women were offered vaccination.

All analyses were performed using SAS version 9.4 (SAS Institute Inc., Cary, NC), using proc glimmix with adaptive Gauss-Hermite quadrature approximation of the maximum likelihood. We used a significance level of $p<.05$. The records with missing data were excluded from the analyses, as these represented less than $5 \%$ of the study population.

\section{Sensitivity analyses}

In sensitivity analyses, we calculated the type-specific and pooled estimates of VE for women who reported to be vaccinated with 3 doses. Moreover, we repeated the stratified analyses, assuming catch-up cohorts were offered vaccination 3 months later, on 31 May 2009 because there was variation in the dates that vaccination was offered during the catchup campaign [28]. 
Table 2.1. Characteristics of the study population and a comparison between vaccinated and unvaccinated women.

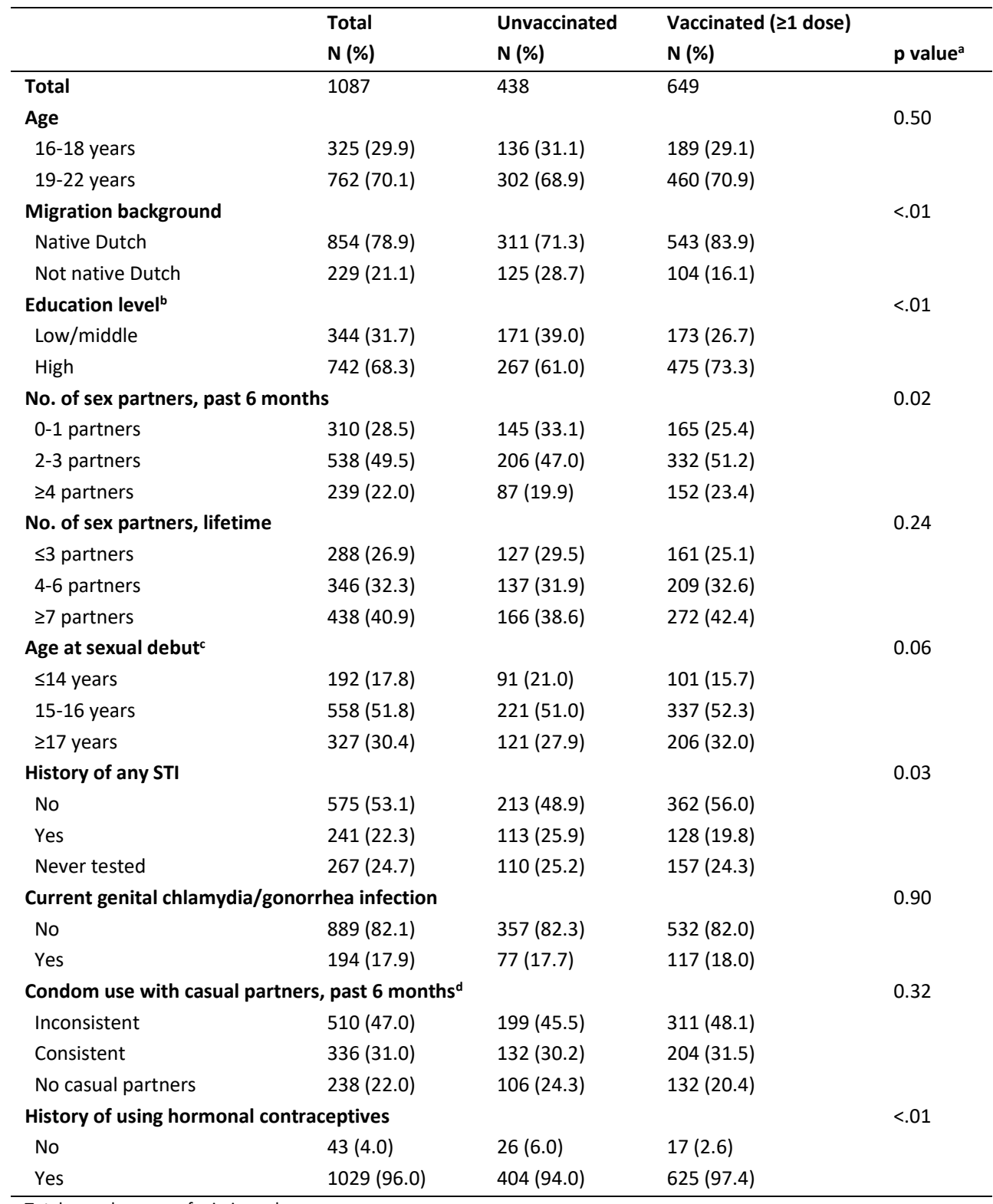

Totals vary because of missing values.

a. Comparing women vaccinated at least once with unvaccinated women.

b. High educational level included school of higher general secondary education, pre-university education, university of applied sciences, and university. Low/middle educational level included all other forms of education.

c. Vaginal or anal intercourse.

d. Inconsistent included reporting never, rarely and "sometimes I do, sometimes I do not" condom use. Consistent included reporting often or always condom use. 


\section{Results}

\section{Study population}

In the PASSYON study years 2011-2015, 1,198 women had been eligible for HPV vaccination, of which 1,087 women reported their vaccination status and provided a cervicovaginal swab (Figure 2.1). Of these 1,087 women, 649 (60\%) reported to be vaccinated and 438 (40\%) reported to be unvaccinated. Of the women who reported to be vaccinated, $70 \%(n=456)$ reported to be vaccinated with 3 doses, $11 \%(n=72)$ reported $<3$ doses, and $19 \%(n=121)$ reported to not know the number of doses. Of the women who reported to be vaccinated, 94\% belonged to the catch-up cohorts (birth cohort 1993-1996).

The characteristics of the study population, stratified by vaccination status, are presented in Table 2.1. Vaccinated women were more often native Dutch and highly educated. They had more partners in the past 6 months, were older at sexual debut, reported less often a history of STIs, and used hormonal contraceptives more often.

\section{Validation of self-reported vaccination status}

In total, $43 \%$ of the study population had serum available for antibody testing. Of the selfreported vaccinated women, 96\% were seropositive for both HPV16 and HPV18. Only 11 self-reported vaccinated women (4.2\%) were seronegative for HPV16 or HPV18 or both (Supplementary Figure 2.1). Of these 11 women, 8 reported 3 doses, $2<3$ doses, and 1 reported not to know the number of doses. The HPV16 and HPV18 antibody concentrations agreed well with the self-reported vaccination status (AUC 92.3\%).

\section{HPV prevalence}

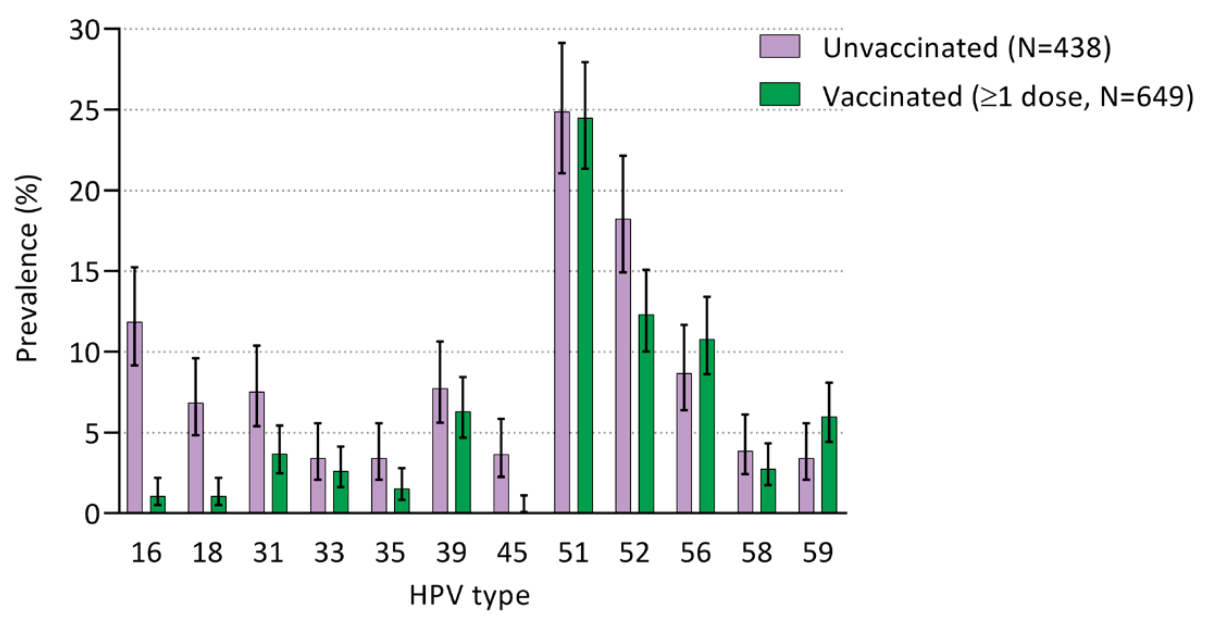

Figure 2.2. High-risk HPV prevalence by vaccination status. 
Overall, $53 \%$ tested positive for $\geq 1 \mathrm{hrHPV}$ type. Of the vaccinated women, $49 \%$ were positive for an hrHPV type compared to $59 \%$ of the unvaccinated women. HPV51 was the most prevalent type followed by HPV52. For most hrHPV types, the prevalence was lower for vaccinated compared to unvaccinated women (Figure 2.2).

\section{Vaccine effectiveness estimates}

The pooled VE against the 2 vaccine types was 89.9\%; $92.3 \%$ against HPV16 and $85.5 \%$ against HPV18 (Figure 2.3). Moreover, we calculated significant VE against the non-vaccine types HPV45, HPV35, HPV31, and HPV52. Although borderline non-significant, the VE against HPV59 was negative (-89\%). The pooled VE against the hrHPV types included in the 9 vHPV vaccine was $60.5 \%$ and against all $12 \mathrm{hrHPV}$ types $32.9 \%$.
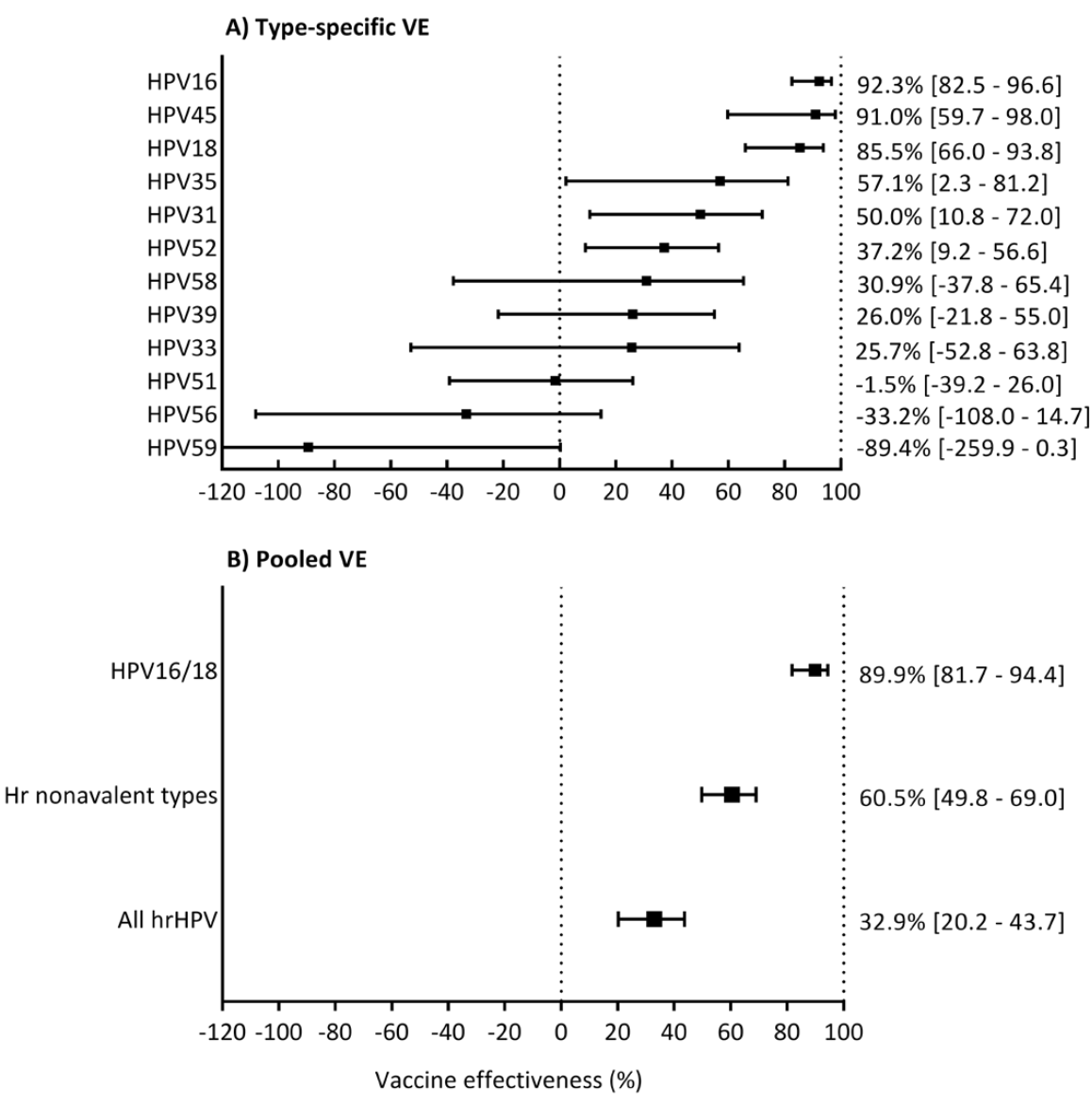

Figure 2.3. VE for at least 1 dose against, A) type-specific hrHPV positivity and B) pooled estimates.

Note: The hr nonavalent HPV types included: HPV16/18/31/33/45/52/58. All hrHPV types included: HPV16/18/31/33/35/39/45/ $51 / 52 / 56 / 58 / 59$. VE was corrected for: migration background, education level, number of sex partners in the past 6 months, age at sexual debut, history of any STI, and history of hormonal contraceptives use. 
Results from the stratified analyses are presented in Table 2.2. Among women who were not sexually active when vaccination was offered, the adjusted pooled VE against the vaccine types (92.2\%) was higher than among women who were (possibly) sexually active when vaccination was offered (81.1\%). Among women who were offered vaccination $5 / 6$ years ago, we observed similar or higher VE against HPV16/18 (92.4\%), the hrHPV types included in the 9vHPV vaccine (65.5\%), and all hrHPV types (34.6\%) compared to women who were offered vaccination $<5$ years ago $(83.2 \%, 50.7 \%$, and $33.0 \%$, respectively).

Table 2.2. VE against pooled estimates, stratified by sexual activity when vaccination was offered and time since vaccination was offered.

\begin{tabular}{|c|c|c|c|c|}
\hline & \multirow[b]{2}{*}{$\mathbf{N}(\%)$} & \multicolumn{3}{|c|}{ VE $(95 \% \mathrm{CI})^{\mathrm{a}}$} \\
\hline & & HPV16/18 & $\begin{array}{l}\text { Hr nonavalent } \\
\text { types }^{b}\end{array}$ & All hrHPV \\
\hline \multicolumn{5}{|c|}{ Women not sexually active when vaccination was offered } \\
\hline Unvaccinated & $303(37.7)$ & & & \\
\hline Vaccinated ( $\geq 1$ dose) & $501(62.3)$ & $92.2(83.2-96.4)$ & $60.1(47.1-70.0)$ & $29.6(13.4-42.7)$ \\
\hline \multicolumn{5}{|c|}{ Women (possibly) sexually active when vaccination was offered } \\
\hline Unvaccinated & $119(47.6)$ & & & \\
\hline Vaccinated ( $\geq 1$ dose) & $131(52.4)$ & $81.1(52.1-92.5)$ & $60.2(36.2-75.2)$ & $39.9(16.3-56.8)$ \\
\hline \multicolumn{5}{|c|}{ Women offered vaccination $<5$ years ago } \\
\hline Unvaccinated & $178(43.1)$ & & & \\
\hline Vaccinated ( $\geq 1$ dose) & $235(56.9)$ & $83.2(57.9-93.3)$ & $50.7(23.9-68.1)$ & $33.0(10.4-49.8)$ \\
\hline \multicolumn{5}{|c|}{ Women offered vaccination $5 / 6$ years ago } \\
\hline Unvaccinated & $244(38.1)$ & & & \\
\hline Vaccinated ( $\geq 1$ dose) & 397 (61.9) & $92.4(83.6-96.5)$ & $65.5(53.9-74.1)$ & $34.6(19.0-47.2)$ \\
\hline \multicolumn{5}{|c|}{$\begin{array}{l}\text { For the catch-up cohorts, vaccination was offered on } 1 \text { March } 2009 \text {. For the cohorts vaccinated in the NIP, vaccination was } \\
\text { offered on } 1 \text { March in the year they turn } 13 \text { years old. } \\
\text { a. VE was corrected for: migration background, education level, number of sex partners in the past } 6 \text { months, age at sexual } \\
\text { debut, history of any STI, history of hormonal contraceptives use, and age vaccination was offered. } \\
\text { b. Including HPV types HPV } 16 / 18 / 31 / 33 / 45 / 52 / 58 \text {. } \\
\text { c. Including HPV types HPV16/18/31/33/35/39/45/51/52/56/58/59. } \\
\text { d. Includes women who reported the same age (in years) of sexual debut as the age they were offered vaccination. }\end{array}$} \\
\hline
\end{tabular}

\section{Sensitivity Analyses}

The VE estimates according to vaccination with 3 doses are presented in Supplementary Figure 2.2. Overall, results were comparable to the main analysis. The pooled VE against the vaccine types was somewhat higher; $94.7 \%$. The negative VE against HPV59 became borderline statistically significant $(-107.2 \%$, 95\% confidence interval $[\mathrm{Cl}]-307.1$ to -5.4$)$. Assuming vaccination for the catch-up cohorts was offered 3 months later did not lead to different results in the stratified analyses (Supplementary Table 2.1). 


\section{Discussion}

We demonstrated high VE from the 2VHPV vaccine against the vaccine types HPV16/18 and significant cross-protection against the hrHPV types 45, 35, 31, and 52. Together, these cross-protective types are associated with approximately an additional $15 \%$ of all cervical cancers [3]. To our knowledge, this is the first observational study reporting VE against hrHPV positivity on a type-specific level for the $2 \mathrm{VHPV}$ vaccine. The cross-protective VE from the $2 \mathrm{vHPV}$ vaccine suggests that the impact of HPV vaccination will be greater than anticipated upon introduction [30].

The high HPV prevalence among STI clinic visitors and sensitive diagnostics to measure infection status, enabled us to measure the type-specific VE against HPV positivity from cross-sectional data. The usefulness of using data from high-risk populations to infer VE in an early stage after the introduction of mass vaccination has been shown by Australian studies; 2 years after HPV vaccination was implemented in Australia, a decline was observed in genital warts among young women and heterosexual men visiting sexual health services [31]. This declining trend was later confirmed in other settings more representative for the general population [32,33].

We do acknowledge some limitations. First, we used self-reported vaccination status, which is prone to recall bias. The vaccination coverage in our study population was comparable to the vaccination coverage in the total Dutch population: $52 \%$ of the catch-up cohorts received 3 doses and this increased to $59 \%$ for birth cohort 1999; an additional $3.8 \%$ received $<3$ doses [34-36]. We showed reliable reporting of vaccination status in our study, but we could only validate self-reported vaccination status among women with serum available. Due to the recent policy changes for syphilis and HIV testing at the STI clinic towards high-risk individuals, women with serum available could be biased towards having higher antibody concentrations [37], complicating the distinction between vaccinated and unvaccinated women. Nevertheless, antibody concentrations performed well in discriminating self-reported vaccination status. Moreover, misclassification according to self-reported vaccination status would lead to conservative estimates of VE. Second, because our study population consisted mainly of women who were vaccinated during the catch-up campaign, some women were probably HPV infected at vaccination, leading to lower VE compared to an HPV-naive population [5, 27]. Indeed, we showed a higher VE against the vaccine types among women with a reported sexual debut after vaccination was offered, in line with results from the vaccine trials. Last, most women in our study were vaccinated according to the 3 -dose schedule as this was the guideline prevailing at the time of vaccination, so our results might not be generalizable to the current 2-dose schedule. In our study, the VE against the vaccine types was higher for 3 doses compared to $\geq 1$ dose, indicating a lower VE among women who did not know the number of doses or who 
reported $<3$ doses. Because of a limited number of women who reported having received 2 doses and because we did not known the interval between doses, we were unable to evaluate the current 2-dose schedule with 6 months between doses.

Our results agree well with the literature. Overall, the VE that we calculated against HPV16/18 positivity and against cross-protective types, are in line with data from the 2vHPV vaccine trials [7]. In the PATRICIA trial, the largest phase III trial, cross-protection has been described against persistent HPV31, 33, 45, 51, and 52 infections and against incident HPV35 infection $[8,9]$. In contrast to the PATRICIA trial, we did not find statistically significant cross-protection against HPV33 or HPV51. In the Costa Rica Vaccine Trial, the efficacy against HPV33 was, like ours, not statistically significant ( $32 \%, 95 \% \mathrm{Cl}-41 \%$ to $68 \%$, against 6-month persistent infection) and against HPV51 negative $(-56 \%, 95 \% \mathrm{Cl}-114 \%$ to $-14 \%$, against 6-month persistent infection) [10]. We found no effect on HPV51. Effect estimates from observational studies against non-vaccine HPV types are still limited. Trend studies found that the HPV31/33/45 prevalence decreased in post-vaccination periods compared to pre-vaccination periods, suggestive of cross-protection [38-40]. Among women who underwent their first cervical screening in Scotland, vaccine effectiveness was observed against HPV31, 33, and 45 [15].

In our study, the VE against the pooled outcomes was similar or even higher among women who were offered vaccination 5 or 6 years ago compared to women who were offered vaccination more recently. These analyses were adjusted for sexual behavior and age when vaccination was offered. These findings are in line with those from Scotland, where high VE against the vaccine types HPV16/18 and against HPV types 31, 33, and 45 was observed up to 7 years after vaccination [15]. Due to low numbers in the stratified analyses, we were unable to calculate the type-specific VE by time since vaccination was offered. As the PASSYON study continues, we will repeat the analyses to investigate the duration of protection further.

We observed a negative VE against HPV59, which was just statistically significant in sensitivity analysis restricted to women who reported 3 doses versus no vaccination. The SPF10-LiPA25 assay that we used in the current study is very sensitive, but the detection limit for HPV59 is much higher than for the other hrHPV types, which could lead to an underestimation of the HPV59 prevalence [41, 42]. Moreover, this assay is a broadspectrum polymerase chain reaction (PCR) in which some competition between types in the same sample can occur [43]. Possibly due to the reduced occurrence of vaccine and crossprotection types, HPV59 was more often detected in vaccinated compared to unvaccinated women, which would lead to an artificial negative VE. This phenomenon of increased detection is referred to as unmasking [44]. Another possible explanation for a negative VE is type-replacement. This means that an HPV type is taking over the vacated ecological niche 
of the vaccine and cross-protective types [44]. In post-hoc analyses of the PATRICIA trial, an alternative HPV DNA testing algorithm was used including a type-specific test that is not affected by competition between types. Using this type-specific test next to the SPF10LiPA25, the number of HPV59 cases roughly doubled, but the vaccine efficacy against HPV59 remained (non-significantly) negative for 12-month persistent infection (-29.2\%) [9]. Because the sensitivity of the SPF10-LiPA25 for HPV59 is limited and because the confidence intervals were large, the negative VE against HPV59 in our study should be interpreted with caution. Further research is necessary to investigate what is causing this negative VE estimate.

To conclude, we showed high VE of the 2VHPV vaccine against HPV16/18 positivity and significant cross-protection against HPV45, HPV35, HPV31, and HPV52 in a Dutch high-risk population. We observed cross-protection against 3 of the 5 additional hrHPV types included in the 9vHPV vaccine. As the cross-protective types HPV45, HPV35, HPV31, and HPV52 are associated with an additional $15 \%$ of all cervical cancer cases, cross-protection of the $2 \mathrm{vHPV}$ vaccine can have a major impact on cancer prevention.

\section{References}

1. Walboomers JM, et al. Human papillomavirus is a necessary cause of invasive cervical cancer worldwide. J Pathol 1999; 189:12-9.

2. Bouvard V, et al. A review of human carcinogens-Part B: biological agents. Lancet Oncol 2009; 10:3212.

3. de Sanjose $S$, et al. Human papillomavirus genotype attribution in invasive cervical cancer: a retrospective cross-sectional worldwide study. Lancet Oncol 2010; 11:1048-56.

4. Plummer $M$, et al. Global burden of cancers attributable to infections in 2012: a synthetic analysis. Lancet Glob Health 2016; 4:e609-16.

5. Paavonen J, et al. Efficacy of human papillomavirus (HPV)-16/18 AS04-adjuvanted vaccine against cervical infection and precancer caused by oncogenic HPV types (PATRICIA): final analysis of a doubleblind, randomised study in young women. Lancet 2009; 374:301-14.

6. de Melker $\mathrm{HE}$, et al. [Introductie van vaccinatie tegen baarmoederhalskanker]. NTvG 2009; 153:65861.

7. Skinner SR, et al. Human papillomavirus (HPV)16/18 ASO4-adjuvanted vaccine for the prevention of cervical cancer and HPV-related diseases. Expert Rev Vaccines 2016; 15:367-87.

8. Wheeler $\mathrm{CM}$, et al. Cross-protective efficacy of HPV-16/18 AS04-adjuvanted vaccine against cervical infection and precancer caused by non-vaccine oncogenic HPV types: 4-year end-of-study analysis of the randomised, double-blind PATRICIA trial. Lancet Oncol 2012; 13:100-10.

9. Struyf $F$, et al. Post hoc analysis of the PATRICIA randomized trial of the efficacy of human papillomavirus type 16 (HPV-16)/HPV-18 AS04adjuvanted vaccine against incident and persistent infection with nonvaccine oncogenic HPV types using an alternative multiplex type-specific PCR assay for HPV DNA. Clin Vaccine Immunol 2015; 22:235-44.

10. Herrero $R$, et al. Prevention of persistent human papillomavirus infection by an HPV16/18 vaccine: a community-based randomized clinical trial in Guanacaste, Costa Rica. Cancer Discov 2011; 1:40819.

11. Woestenberg PJ, et al. No evidence for crossprotection of the HPV-16/18 vaccine against HPV6/11 positivity in female STI clinic visitors. J Infect 2017; 74:393-400. 
12. Mollers $M$, et al. Effectiveness of human papillomavirus vaccine against incident and persistent infections among young girls: Results from a longitudinal Dutch cohort study. Vaccine 2015; 33:2678-83.

13. Cuschieri K, et al. Impact of partial bivalent HPV vaccination on vaccine-type infection: a populationbased analysis. Br J Cancer 2016; 114:1261-4.

14. Kumakech E, et al. Significantly Reduced Genoprevalence of Vaccine-Type HPV-16/18 Infections among Vaccinated Compared to NonVaccinated Young Women 5.5 Years after a Bivalent HPV-16/18 Vaccine (Cervarix(R)) Pilot Project in Uganda. PLoS One 2016; 11:e0160099.

15. Kavanagh K, et al. Changes in the prevalence of human papillomavirus following a national bivalent human papillomavirus vaccination programme in Scotland: a 7-year cross-sectional study. Lancet Infect Dis 2017; 17:1293-302.

16. Pollock KG, et al. Reduction of low- and highgrade cervical abnormalities associated with high uptake of the HPV bivalent vaccine in Scotland. Br J Cancer 2014; 111:1824-30.

17. Jit $\mathrm{M}$, et al. Comparing bivalent and quadrivalent human papillomavirus vaccines: economic evaluation based on transmission model. BMJ 2011; 343:d5775.

18. Brisson $\mathrm{M}$, et al. Comparative cost-effectiveness of the quadrivalent and bivalent human papillomavirus vaccines: a transmission-dynamic modeling study. Vaccine 2013; 31:3863-71.

19. Joura EA, et al. A 9-valent HPV vaccine against infection and intraepithelial neoplasia in women. $\mathrm{N}$ Engl J Med 2015; 372:711-23.

20. Vriend $\mathrm{HJ}$, et al. Type-specific human papillomavirus infections among young heterosexual male and female STI clinic attendees. Sex Transm Dis 2012; 39:72-8.

21. Vriend $\mathrm{HJ}$, et al. Patterns of human papillomavirus DNA and antibody positivity in young males and females, suggesting a site-specific natural course of infection. PLoS One 2013; 8:e60696.

22. Scherpenisse $M$, et al. Seroprevalence of seven high-risk HPV types in The Netherlands. Vaccine 2012; 30:6686-93.

23. Woestenberg PJ, et al. Comparison of STI-related consultations among ethnic groups in the
Netherlands: an epidemiologic study using electronic records from general practices. BMC Fam Pract 2015; 16:70.

24. Auranen $\mathrm{K}$, et al. Colonisation endpoints in Streptococcus pneumoniae vaccine trials. Vaccine 2013; 32:153-8.

25. Xue $X$, et al. Marginal and mixed-effects models in the analysis of human papillomavirus natural history data. Cancer Epidemiol Biomarkers Prev 2010; 19:159-69.

26. Halloran ME, et al. Design and Analysis of Vaccine Studies. New York: Springer, 2010 (Gail M, Krickeberg K, Samet J, Tsiatis A, Wong W, eds. Statistics for Biology and Health).

27. Lehtinen $M$, et al. Overall efficacy of HPV-16/18 AS04-adjuvanted vaccine against grade 3 or greater cervical intraepithelial neoplasia: 4-year end-ofstudy analysis of the randomised, double-blind PATRICIA trial. Lancet Oncol 2012; 13:89-99.

28. Rondy $M$, et al. Determinants for HPV vaccine uptake in the Netherlands: A multilevel study. Vaccine 2010; 28:2070-5.

29. Malagon T, et al. Cross-protective efficacy of two human papillomavirus vaccines: a systematic review and meta-analysis. Lancet Infect Dis 2012; 12:781-9. 30. Bogaards JA, et al. Long-term impact of human papillomavirus vaccination on infection rates, cervical abnormalities, and cancer incidence. Epidemiology 2011; 22:505-15.

31. Donovan B, et al. Quadrivalent human papillomavirus vaccination and trends in genital warts in Australia: analysis of national sentinel surveillance data. Lancet Infect Dis 2011; 11:39-44.

32. Harrison C, et al. Decreased management of genital warts in young women in Australian general practice post introduction of national HPV vaccination program: results from a nationally representative cross-sectional general practice study. PLoS One 2014; 9:e105967.

33. Smith MA, et al. Fall in genital warts diagnoses in the general and indigenous Australian population following implementation of a national human papillomavirus vaccination program: analysis of routinely collected national hospital data. J Infect Dis 2015; 211:91-9. 
34. van Lier EA, et al. [Vaccinatiegraad Rijksvaccinatieprogramma Nederland: Verslagjaar 2011]. Bilthoven: RIVM, 2011. Nr: 210021014/2011. 35. van Lier EA, et al. [Vaccinatiegraad Rijksvaccinatieprogramma Nederland: Verslagjaar 2011]. Bilthoven: RIVM, 2016. Nr: 2016-0064.

36. van Lier EA, et al. [Vaccinatiegraad Rijksvaccinatieprogramma Nederland: Verslagjaar 2011]. Bilthoven: RIVM, 2012. Nr: 201001001/2012. 37. de Araujo-Souza PS, et al. Determinants of baseline seroreactivity to human papillomavirus type 16 in the Ludwig-McGill cohort study. BMC Infect Dis 2014; 14:578.

38. Drolet $\mathrm{M}$, et al. Population-level impact and herd effects following human papillomavirus vaccination programmes: a systematic review and meta-analysis. Lancet Infect Dis 2015; 15:565-80.

39. Cameron RL, et al. Human Papillomavirus Prevalence and Herd Immunity after Introduction of Vaccination Program, Scotland, 2009-2013. Emerg Infect Dis 2016; 22:56-64.
40. Mesher D, et al. Continuing reductions in HPV $16 / 18$ in a population with high coverage of bivalent HPV vaccination in England: an ongoing crosssectional study. BMJ Open 2016; 6:e009915.

41. van Alewijk $D$, et al. A human papilloma virus testing algorithm comprising a combination of the L1 broad-spectrum SPF10 PCR assay and a novel E6 high-risk multiplex type-specific genotyping PCR assay. J Clin Microbiol 2013; 51:1171-8.

42. Labo Bio-medical Products BV (LBP). DNA ELISA kit HPV SPF10, version 1. Rijswijk: Labo Bio-medical Products BV, 2016. Nr: KI0022 v5.2.

43. van Doorn $L$, et al. Highly effective detection of human papillomavirus 16 and 18 DNA by a testing algorithm combining broad-spectrum and typespecific PCR. J Clin Microbiol 2006; 44:3292-8.

44. Tota JE, et al. Epidemiologic approaches to evaluating the potential for human papillomavirus type replacement postvaccination. Am J Epidemiol 2013; 178:625-34. 


\section{Supplementary information to Chapter 2}

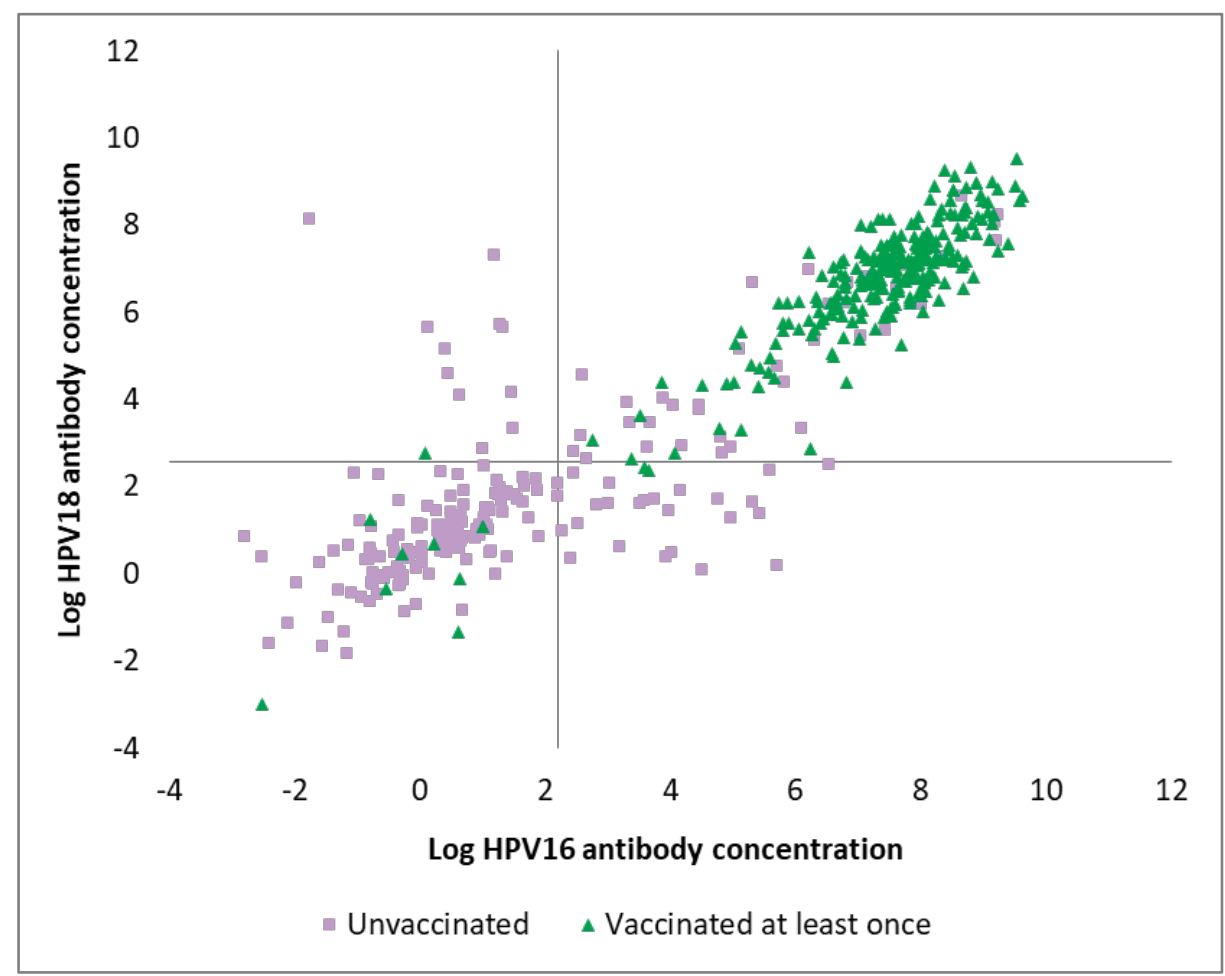

Supplementary Figure 2.1. Log HPV16 and log HPV18 antibody concentration by self-reported vaccination status, among women with serum available.

Note: The dashed lines represent the cut-off levels for seropositivity for HPV16 and HPV18. 


\section{A) Type-specific VE}

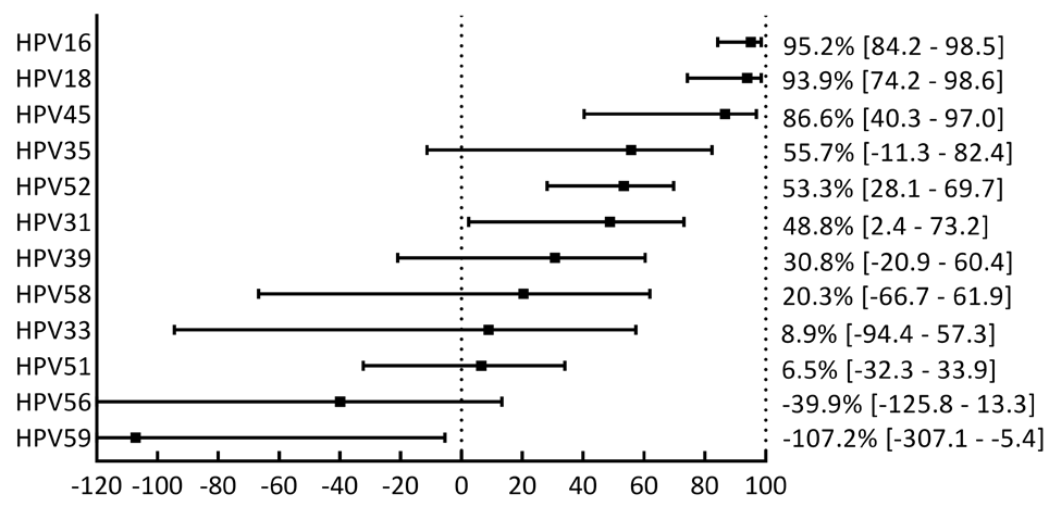

B) Pooled VE

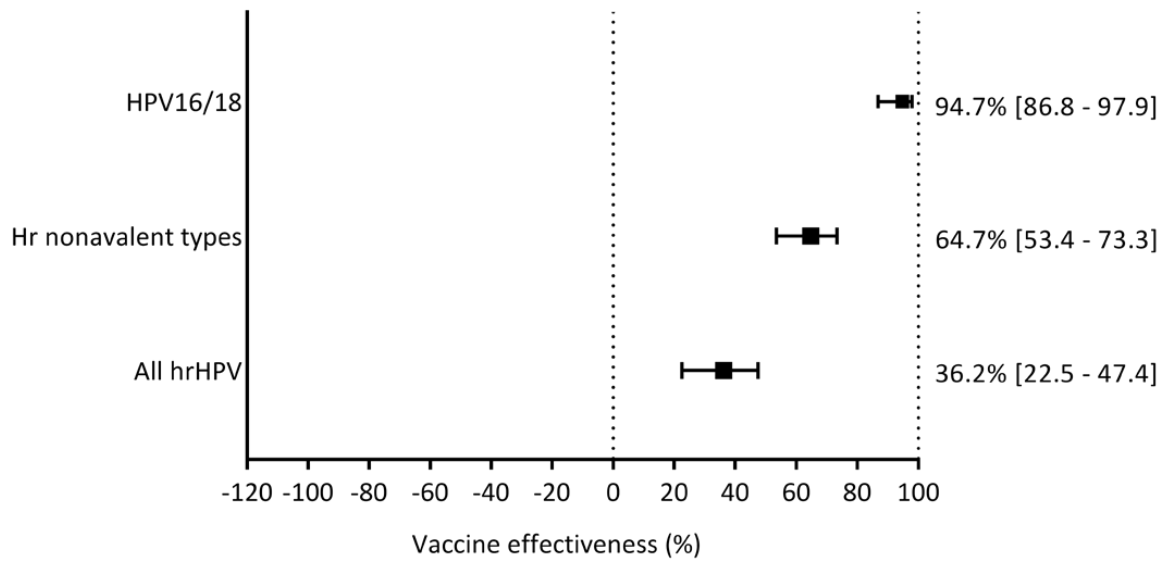

Supplementary Figure 2.2. Sensitivity analyses: VE for 3 doses against, A) type-specific hrHPV positivity and B) pooled estimates.

Note: The hr nonavalent HPV types included: HPV16/18/31/33/45/52/58. All hrHPV types included: HPV16/18/31/33/35/39/45/ 51/52/56/58/59. VE was corrected for: migration background, education level, number of sex partners in the past 6 months, age at sexual debut, history of any STI, and history of hormonal contraceptives use. 
Supplementary Table 2.1. Sensitivity analyses: VE against pooled estimates, stratified by sexual activity when vaccination was offered and time since vaccination was offered, assuming catch-up cohorts were offered vaccination on 31 May 2009.

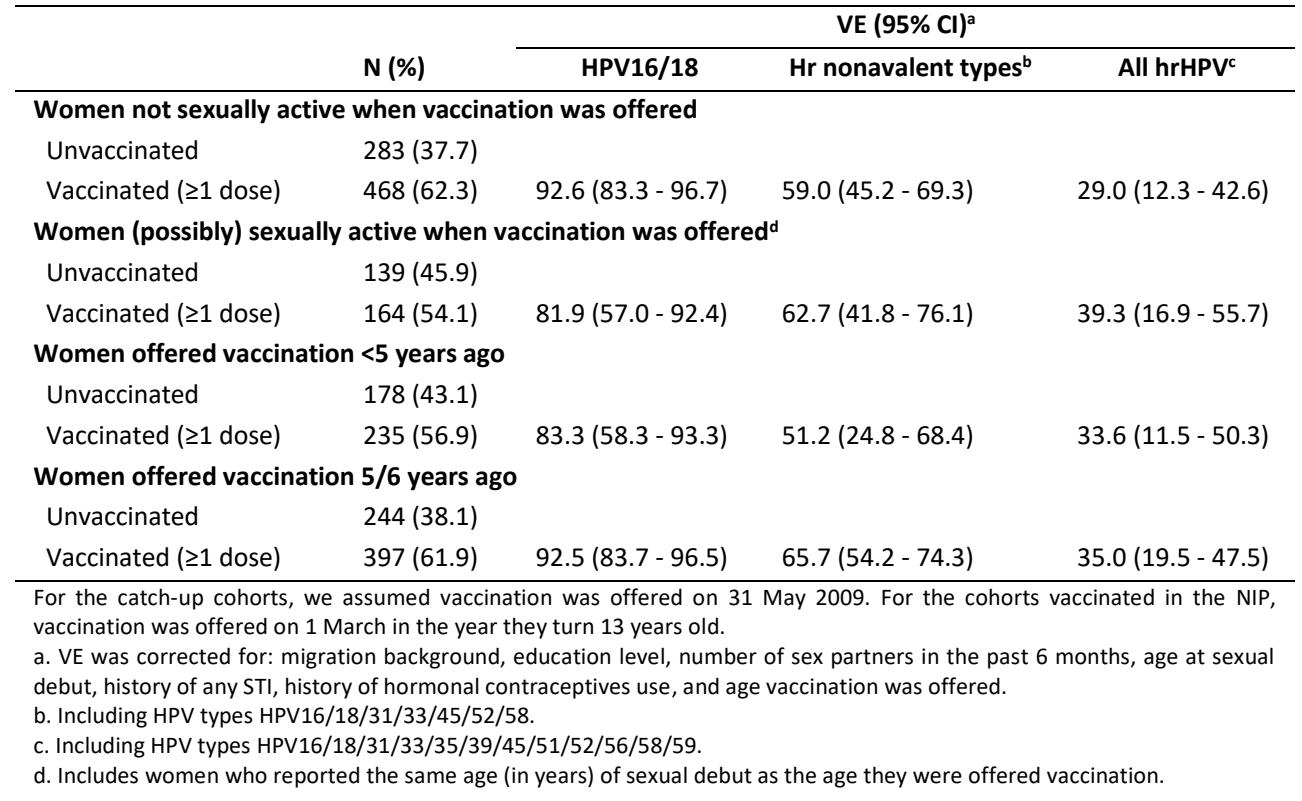






\section{Chapter 3}

\section{Bivalent human papillomavirus (HPV) vaccine effectiveness correlates with phylogenetic distance from HPV vaccine types 16 and 18}

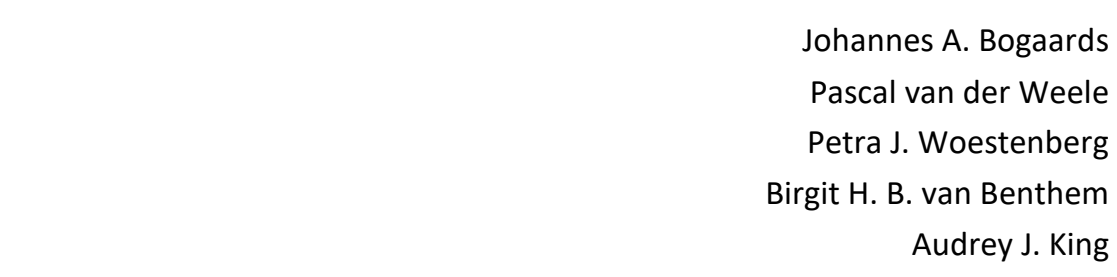

The contents of this chapter have been published in The Journal of Infectious Diseases

J Infect Dis. 2019; 220:1141-6 


\section{Abstract}

To substantiate cross-protection reported across AS04-adjuvanted bivalent human papillomavirus (HPV) vaccine (2vHPV) studies, we re-evaluated vaccine effectiveness against type-specific HPV positivity as a function of phylogenetic distance to vaccine target types HPV16 and 18. We provide evidence of sustained cross-protection up to 8 years postvaccination in a high-risk population in the Netherlands. Moreover, our findings suggest that genomic distance better explains cross-protection than distance measures based on capsid antigens only. Taken together, $2 \mathrm{vHPV}$ is predicted to provide partial cross-protection against HPV31, 33, 35, 45, 52, and possibly 58, that is, acknowledged oncogenic types with close phylogenetic relationships to HPV16 or 18. 


\section{Introduction}

The sexually transmitted human papillomavirus (HPV) is considered a necessary factor for development of cervical cancer and is linked to other anogenital and oropharyngeal cancer [1]. Papillomaviruses are characterized by genotype, defined as $>10 \%$ DNA sequence divergence from other known genotypes (generally termed "types") in the L1 capsid gene [2]. Most HPV-related malignancies are attributable to types 16 and 18. Consequently, firstgeneration vaccines, based on recombinant expression of L1 in systems yielding virus-like particles (VLPS), focused on HPV16 and 18, with the quadrivalent (4VHPV) vaccine also containing L1 VLPS of HPV6 and 11, primarily associated with anogenital warts. As up to $30 \%$ of cervical cancer is attributed to oncogenic types other than 16 or 18, achieving broader protection through cross-reactivity or expansion of the range of VLP types is desirable.

Endeavors to expand the range of VLP types have resulted in the second-generation nonavalent HPV (9vHPV) vaccine, containing L1 VLPs from those already contained in the 4vHPV vaccine plus the 5 next most common types in cervical cancer: HPV types 31, 33, 45, 52 , and 58 . Alternatively, the minor capsid protein $L 2$, though less immunogenic than $L 1$, is potentially an effective target for prophylaxis, as several subdominant protective epitopes of $L 2$ are well conserved between types and broadly cross-protective in animal models. By contrast, the protection elicited by L1 VLPs is generally taken to be type-restricted (i.e. reactive with the homologous type) [1].

First-generation HPV vaccines have shown durable type-specific protection for at least a decade [3]. Importantly, this protection is not absolutely type-restricted, because significant cross-protection has been observed against several non-vaccine types, particularly for the AS04-adjuvanted bivalent (2vHPV) vaccine containing L1 VLPS of HPV16 and 18 only. In the largest phase III trial of the 2vHPV vaccine, cross-protection was described against persistent HPV6, 31, 33, 45, 51, and 52 infections, and against incident HPV35 infection. However, findings with regard to non-vaccine types are equivocal, as the 2 VHPV vaccine trial from Costa Rica reported significant protection against HPV31, 45, and 52, insignificant protection against HPV33, and no effect on HPV51 [4].

Recent population-based studies from the United Kingdom and the Netherlands confirm some cross-protection from 2vHPV in post-vaccine surveillance. In Scotland, a decrease in the prevalence of HPV31, 33, and 45 was observed among women who underwent their first cervical screening within 7 years after initiating a 2vHPV vaccination program [5]. In the Netherlands, significant cross-protection was estimated against HPV31, 35, 45, and 52 among female visitors to sexually transmitted infection (STI) clinics who reported to be vaccinated, relative to vaccine-eligible controls [6]. Cross-protection from 2vHPV against HPV6 or 11 has not been replicated in post-vaccine surveillance, neither in England [7] nor in the Netherlands [8]. 
To reconcile the inconsistencies in cross-protection reported across $2 \mathrm{vHPV}$ vaccine studies and to assess the type-restricted nature of the protection elicited by L1 VLPS, we reevaluated vaccine effectiveness (VE) against type-specific HPV positivity among STI clinic visitors up to 8 years after vaccination as a function of phylogenetic distance to L1 capsid antigens contained in the $2 \mathrm{vHPV}$ vaccine.

\section{Methods}

We estimated VE from the PASSYON study, a biennial cross-sectional survey in the Netherlands, as described before [6], but now with an extra study round and including all genotypes in the SPF10-LiPA25 assay (DDL Diagnostics Laboratory). In brief, women aged 16-24 years, who had been eligible for HPV vaccination and visited the STI clinic between 2011 and 2017, provided a cervicovaginal swab that was analyzed using a polymerase chain reaction (PCR-) based assay able to detect 25 HPV types, including 12 acknowledged and 3 possibly oncogenic types (Table 3.1). We compared type-specific HPV positivity between 1,305 self-reported vaccinated ( $\geq 1$ dose) and 799 unvaccinated women. The self-reported vaccination status was validated by serology among those who also provided blood.

Phylogenetic distance of each genotype to the 2vHPV types used for construction of VLPS was calculated from reference DNA sequences obtained via the papillomavirus episteme, a database of curated papillomavirus genomic sequences [9]. We performed a phylogenetic analysis on $\mathrm{L} 1$ amino acid (AA) composition using a general Dayhoff matrix for evolutionary change in L1 protein with standard codon model and multiple sequence alignment (www.ebi.ac.uk/Tools/msa/muscle/). In addition, we constructed phylogenetic trees directly from DNA sequences on the basis of $\mathrm{L} 1$ capsid gene, and on the basis of wholegenome sequences (WGS). Unrooted evolutionary trees from L1 protein or DNA sequences were constructed by maximum likelihood with substitution model selection using IQ-TREE version 1.6.0 software (www.iqtree.org/). Phylogenetic distance was calculated from the consensus tree constructed from 1000 ultra-fast bootstrap trees [10]. Finally, we compared dependence on phylogenetic distance to the Hamming distance from aligned L1 sequences, that is, the number of positions at which the corresponding L1 proteins of reference types are different from the AA expressed by VLPs in 2vHPV.

We assessed VE as a function of minimum distance to VLP AA composition in L1 protein analysis and as a function of minimum distance to HPV16 or 18 reference sequences in DNA analyses. Because the LiPA25 assay cannot distinguish between types 68/73/97, we omitted these types from statistical analysis. We also omitted HPV59, as the estimate of crossprotection against this type is potentially hampered by technical issues in the assay [6]. For the remaining types, we fitted a penalized regression spline to the estimates from the 
logistic mixed model, weighted by the square root of the number of positives used in VE estimation (Table 3.1), as a function of phylogenetic distance. The smoothness of the function was determined by general cross-validation, and confidence intervals ( $\mathrm{Cls}$ ) were obtained through Bayesian approximation. In addition, we performed weighted covariance analyses on the rank values of the various distance measures, stratified by (putative) oncogenicity of HPV types. Statistical analyses were performed with R version 3.5.1.

Table 3.1. Bivalent HPV VE against type-specific HPV positivity.

\begin{tabular}{|c|c|c|c|c|}
\hline HPV type ${ }^{a}$ & VE $(95 \% \mathrm{Cl})^{b}$ & $\begin{array}{l}\text { N (n vaccinated / } \\
\text { n nonvaccinated)c }\end{array}$ & Reference genome $^{d}$ & $\begin{array}{l}\text { Hamming } \\
\text { distance }^{\mathrm{e}}\end{array}$ \\
\hline \multicolumn{5}{|l|}{ High-risk } \\
\hline 16 & $0.92(0.86-0.96)$ & $100(13 / 87)$ & gi|333031||lc||HPV16REF.1 & 37 \\
\hline 18 & $0.89(0.7-80.94)$ & $63(11 / 52)$ & gi|60975||cl|HPV18REF.1 & 41 \\
\hline 31 & $0.66(0.51-0.77)$ & $129(50 / 79)$ & gi|333048||cl|HPV31REF.1 & 102 \\
\hline 33 & $0.41(0.05-0.63)$ & $73(37 / 36)$ & gi|333049||cl|HPV33REF.1 & 111 \\
\hline 35 & $0.40(-0.03-0.65)$ & $55(28 / 27)$ & gi|396997||lc||HPV35REF.1 & 105 \\
\hline 39 & $0.15(-0.19-0.39)$ & $165(98 / 67)$ & gi|333245|lc||HPV39REF.1 & 128 \\
\hline 45 & $0.81(0.55-0.92)$ & $28(7 / 21)$ & gi|397022|Icl|HPV45REF.1 & 91 \\
\hline 51 & $-0.24(-0.54-0.01)$ & $522(345 / 177)$ & gi|333087||cl|HPV51REF.1 & 180 \\
\hline 52 & $0.36(0.19-0.50)$ & $342(185 / 157)$ & gi|397038|lcl|HPV52REF.1 & 117 \\
\hline 56 & $-0.17(-0.59-0.14)$ & $220(145 / 75)$ & gi|397053||cl|HPV56REF.1 & 173 \\
\hline 58 & $0.30(-0.06-0.54)$ & $95(52 / 43)$ & gi|222386|lcl|HPV58REF.1 & 113 \\
\hline 59 & $-0.95(-2.17$ to -0.20$)$ & $96(73 / 23)$ & gi|557236|lc||HPV59REF.1 & 125 \\
\hline \multicolumn{5}{|c|}{ Probable high-risk } \\
\hline 53 & $0.26(0.05-0.43)$ & $332(189 / 143)$ & gi|9627377||lc||HPV53REF.1 & 173 \\
\hline 66 & $0.02(-0.26-0.24)$ & $340(212 / 128)$ & gi|1020290|lcl|HPV66REF.1 & 174 \\
\hline \multirow[t]{3}{*}{$68 / 73 / 97$} & $-0.08(-0.63-0.28)$ & $110(71 / 39)$ & gi|71726685||cl|HPV68REF.1 & 139 \\
\hline & & & gi|1491692 |lcl|HPV73REF.1 & 150 \\
\hline & & & gi|71726694||cl|HPV97REF.1 & 89 \\
\hline \multicolumn{5}{|l|}{ Low-risk } \\
\hline 6 & $-0.15(-0.52-0.14)$ & $263(172 / 91)$ & gi|60955||cl|HPV6REF.1 & 161 \\
\hline 11 & $-0.07(-1.00-0.42)$ & $45(29 / 16)$ & gi|333026|lc||HPV11REF.1 & 162 \\
\hline 34 & $0.00(-1.57-0.61)$ & $19(12 / 7)$ & gi|9627334|Icl|HPV34REF.1 & 154 \\
\hline 40 & $0.08(-0.68-0.50)$ & $46(28 / 18)$ & gi|397014||cl|HPV40REF.1 & 163 \\
\hline 42 & $-1.27(-3.34$ to -0.19$)$ & $57(45 / 12)$ & gi|333211||cl|HPV42REF.1 & 160 \\
\hline 43 & $-0.78(-2.13$ to -0.01$)$ & $67(50 / 17)$ & gi|40804474||lcl|HPV43REF.1 & 176 \\
\hline 44 & $-0.32(-1.30-0.24)$ & $61(42 / 19)$ & gi|1020242|Icl|HPV44REF.1 & 162 \\
\hline 54 & $-0.34(-0.95-0.08)$ & $141(97 / 44)$ & gi|9628437||lc||HPV54REF.1 & 143 \\
\hline 70 & $0.02(-1.03-0.52)$ & $32(20 / 12)$ & gi|1173493|lcl|HPV70REF.1 & 126 \\
\hline 74 & $0.26(-0.19-0.54)$ & $75(42 / 33)$ & gi|27462483|lcl|HPV74REF.1 & 168 \\
\hline
\end{tabular}

a. HPV genotypes in the SPF10-LiPA25 assay, with 68 being indistinguishable from 73 and 97.

b. VE (with $95 \% \mathrm{Cl}$ ) was calculated as 1 minus the adjusted odds ratio from a logistic mixed model described in [6].

c. No. of positive test results (among 1305 vaccinated; 799 non-vaccinated women) used in VE estimation.

d. Whole-genome reference DNA sequences obtained from the papillomavirus genome database (https://pave.niaid.nih.gov/).

e. Minimum number of different amino acids between aligned L1 sequences of reference types and VLPs in bivalent vaccine. 


\section{Results}

Type-specific VE estimates are provided in Table 3.1. The pooled VE against the 2 vaccine types was $91.0 \%$ (95\% Cl, 86.0\%-94.2\%). Pooled VE against all (possibly) oncogenic types was $25.8 \%$ (95\% Cl, $17.7 \%-33.2 \%)$, whereas the pooled VE against non-oncogenic types included in the assay was $-4.9 \%(95 \% \mathrm{Cl},-20.7 \%$ to $8.8 \%)$. We found no indications for dependency of VE on time since vaccination in stratified analyses, comparing women who were offered vaccination $<5$ years ago, 5-6 years ago, or 7-8 years ago (Supplementary Table 3.1).

Overall, there was a clear relationship between VE and phylogenetic distance in L1 protein analysis (Figure 3.1A). The consistently high cross-protection reported for HPV45 is due to its close relationship to HPV18 (Figure 3.1B). The cross-protection of around $50 \%$ against HPV31 and 35 fits their almost equidistant relationship to HPV16. Likewise, the cross-protection of around 35\% against HPV33, 52, and 58 is in line with these types having approximately similar phylogenetic distance to HPV16. Our analysis further supports the notion that the estimate of VE against HPV59 appears to be an outlier.

\section{A) VE as a function of phylogenetic distance}

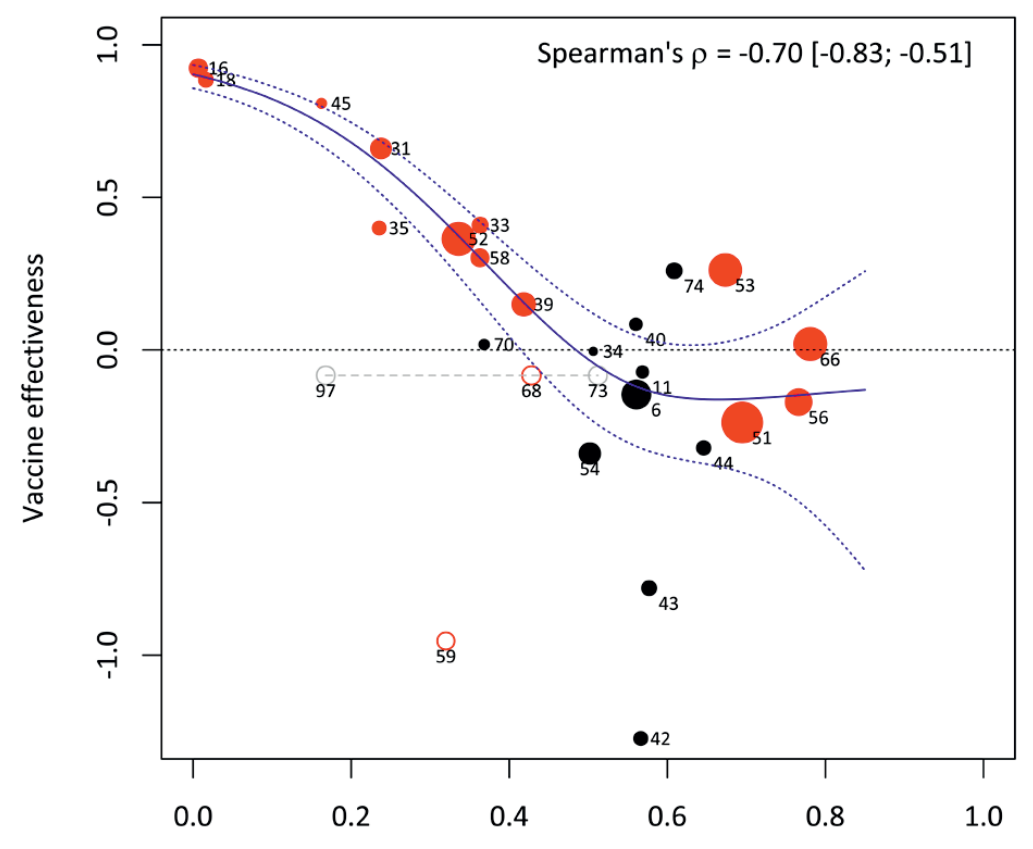

Phylogenetic distance to L1 VLP (protein analysis) 


\section{B) Phylogenetic tree}

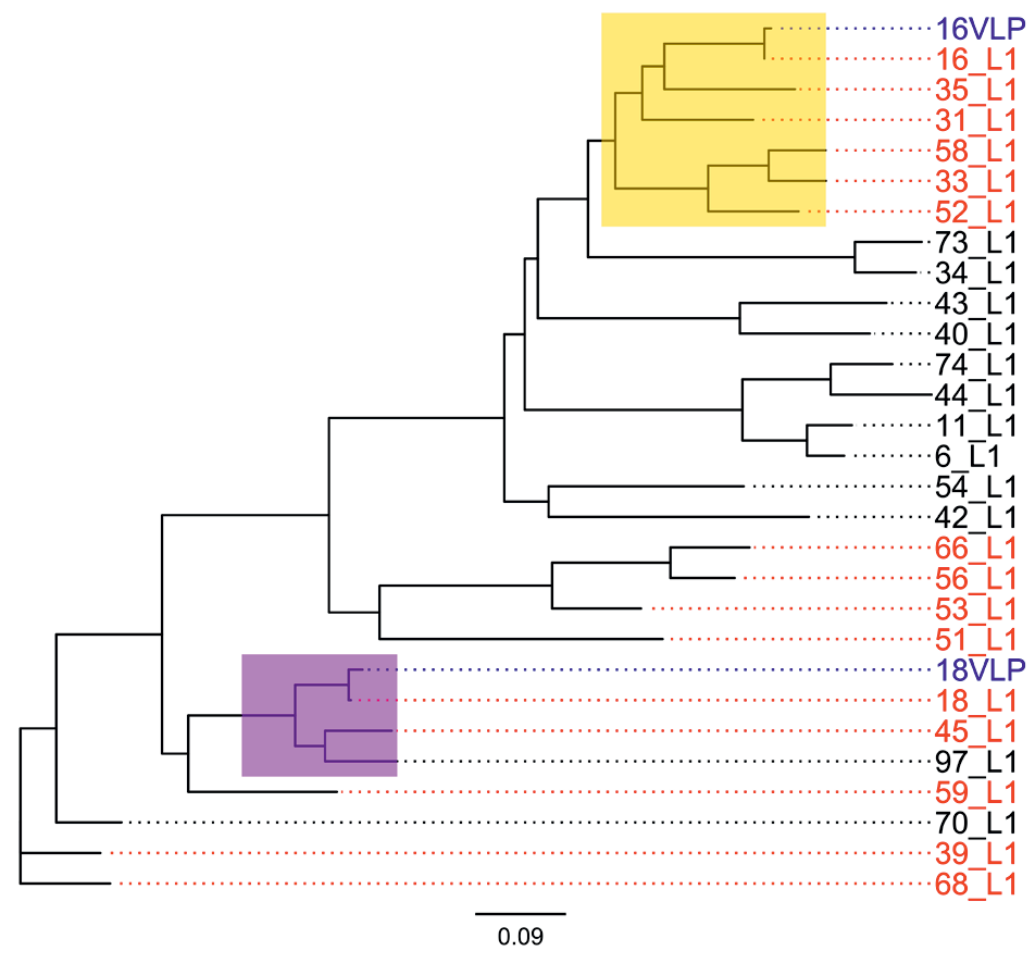

Amino acid substitutions per 100 residues

Figure 3.1. A) Bivalent HPV VE as a function of phylogenetic distance to L1 VLPs and B) phylogenetic tree based on L1 protein.

Note: The VE was calculated from cross-sectional prevalence data [6] for all genotypes in the SPF10-LiPA25 assay. Phylogenetic distance to L1 VLPs was calculated from reference DNA sequences, using the Dayhoff model for evolutionary change in L1 protein. Substitution rate heterogeneity among alignment sites was incorporated by assuming $\gamma$-distributed rates plus a fraction of invariable sites. A) Red data points denote (possibly) oncogenic types, with HPV68 being indistinguishable from HPV73 and 97 (in gray). The size of each data point is plotted proportional to the number of positive test results ( $n$ ) used in VE estimation. Spearman rank correlation (with $95 \%$ confidence limits in brackets) was calculated from all data points weighted by Vn, excluding HPV 59 and $68 / 73 / 97$ (open circles). The estimated spline function (in blue) is shown with $95 \%$ credible intervals (dotted lines). B) Phylogenetic tree based on L1 protein, with blue tips denoting reference sequences used for construction of L1 VLPs in the bivalent HPV vaccine and red tips denoting (possibly) oncogenic types in the SPF10-LiPA25 assay. The yellow and purple clades highlight types that are close enough to L1 VLPs to benefit (in principle) from cross-protection, according to predictions with $95 \%$ confidence from the weighted penalized regression spline in A).

Of the acknowledged oncogenic types, HPV51, 56, and 66 are most distantly related to either L1 VLP and least likely to be affected by cross-neutralizing antibodies induced by the $2 \mathrm{vHPV}$ vaccine (Supplementary Figure 3.1A). The significant rank correlation $(\rho=-0.70[95 \%$ $\mathrm{Cl},-0.83$ to -0.51 ) between VE and phylogenetic distance to L1 VLP in protein analysis was entirely explained by oncogenic types ( $\rho=-0.93[95 \% \mathrm{Cl},-0.95$ to -0.89$]$ ), as no significant correlation was observed for non-oncogenic types (Supplementary Figure 3.1B). 
Analysis based on Hamming distance toward L1 VLPS yielded similar results as L1 phylogenetic analysis (Supplementary Figure 3.2A). Phylogenetic analysis based on the L1 capsid gene and WGS yielded slightly different depictions but was still comparable to those for L1 protein (Supplementary Figure 3.2B and 3.2C). To express the specific association between VE and each phylogenetic distance while controlling for the effect of other measures, we computed their partial rank correlations from the inverse weighted covariance matrix. Apparently, WGS phylogenetic distance to HPV16 or 18 was the strongest independent determinant of VE $\left(\rho_{\text {partial }}=-0.53, p<.01\right)$, with HPV51, 53, 56, and 66 located around the threshold genomic distance still informative for $V E$. The non-oncogenic types were further distanced from vaccine target types in WGS analysis than in analyses based on capsid antigen only, and the partial rank correlations between VE and L1 distance measures were no longer significant when corrected for genomic distance (Supplementary Table 3.2).

\section{Discussion}

This study provides evidence of sustained cross-protection from the $2 \mathrm{vHPV}$ vaccine up to 8 years post-vaccination in a high-risk population. Taken together, the $2 \mathrm{vHPV}$ vaccine is predicted to provide partial cross-protection against HPV types $31,33,35,45,52$, and possibly 58, that is, high-risk types belonging to HPV $\alpha-7$ (including HPV18) or $\alpha-9$ (including HPV16) species [2]. Of those, HPV35 and 58 are not frequently reported among the crossprotective types, which may be due to their relative rarity as compared to other crossprotective types. Likewise, VE against HPV35 and 58 was not significantly different from zero in this study, and regression analyses based on phylogenetic distance predicted only small to moderate effect size. Of the other (possibly) oncogenic types, cross-protection may extend to HPV39 but is unlikely for HPV51, 53, 56, and 66 (i.e. high-risk types belonging to HPV $\alpha-5$ or $\alpha-10$ species) [2]. Similarly, cross-protection against low-risk types was not observed in our study [8] and is not to be expected on the basis of phylogenetic analyses.

Although there has been concern about the durability of cross-reactivity, so far there is no evidence for the waning of cross-protection from the $2 \mathrm{vHPV}$ vaccine in women who have been vaccinated 3 times, as per initial recommendation $[5,6,11]$. Moreover, although we have previously shown reliable reporting of vaccination status in our study [6], our VE estimates and their relation with phylogenetic distance may be underestimated by nondifferential misclassification with regard to self-reported vaccination status. It remains to be seen whether cross-reactivity of the $2 \mathrm{vHPV}$ vaccine following vaccination with $<3$ doses induces similar, long-lasting cross-protection. Analysis of the impact of $2 \mathrm{vHPV}$ 
vaccination among female teenagers in the United Kingdom shows evidence of type-specific protection, but not cross-protection following a single dose of vaccine [12].

One possibility as to why cross-protection is better explained by genomic distance than by measures based on the L1 capsid protein is that L1 VLP may induce cross-neutralizing antibodies to $L 2$ that are critical in preventing viral entry into the host cell [1]. Moreover, the adjuvant ASO4 in the $2 \mathrm{vHPV}$ vaccine has been suggested to induce a T-cell response that enhances local innate control and provides help for subsequent adaptive immunity [13]. Although the mechanisms of adaptive immunity are still ambiguous, it is worthwhile to point out that $2 \mathrm{VHPV}$ vaccination results not only in reduced incidence rates, but also in reduced viral load in breakthrough infections [14], suggesting that VE extends to control of infection post-acquisition. ASO4 is particularly effective in activating antigen-presenting cells, inducing cytokines and a T-helper 1-type response, leading to inhibition of viral transcription or translation [1]. Such features could contribute to cross-protective humoral and cellular control of HPV infections, and may be boosted by natural exposure to nonvaccine HPV types.

Cross-protection can be expected to mitigate the potential for type-replacement by acting as a substitute of latent competitive pressures induced by vaccine types. Thus, oncogenic types that do not benefit from cross-reactivity should be considered foremost in evaluating type-replacement in the wake of vaccination. In this context, the negative VE against HPV59 might stem from differential sensitivity of the SPF10-LiPA25 assay in vaccinees relative to non-vaccinated controls [6], and not from type-replacement. A Finnish community randomized trial on the population effects of $2 \mathrm{vHPV}$ vaccination, using a different PCR-based assay than ours, found no indications for type-replacement by HPV59 [15]. Instead, HPV39 and HPV51 were marked as potential culprits for an increased postvaccination occurrence.

To summarize, our analysis indicates that cross-protection from the $2 \mathrm{vHPV}$ vaccine is sustained up to 8 years post-vaccination and that the level of protection correlates with genomic distance to HPV16 or 18. This suggests that the benefits of 2vHPV vaccination may extend to clinically relevant non-vaccine types, given that oncogenic potential of papillomaviruses itself has a phylogenetic basis [1-3]. Further studies will reveal to what extent cross-protection induced by the $2 \mathrm{VHPV}$ vaccine will contribute to HPV-related disease prevention. 


\section{References}

1. Roden RBS, Stern PL. Opportunities and challenges for human papillomavirus vaccination in cancer. Nat Rev Cancer 2018; 18:240-54.

2. de Villiers EM. Cross-roads in the classification of papillomaviruses. Virology 2013; 445:2-10.

3. Harper DM, DeMars LR. HPV vaccines - A review of the first decade. Gynecol Oncol 2017; 146:196-204.

4. Skinner SR, et al. Human papillomavirus (HPV)16/18 AS04-adjuvanted vaccine for the prevention of cervical cancer and HPV-related diseases. Expert Rev Vaccines 2016; 15:367-87.

5. Kavanagh $\mathrm{K}$, et al. Changes in the prevalence of human papillomavirus following a national bivalent human papillomavirus vaccination programme in Scotland: a 7-year cross-sectional study. Lancet Infect Dis 2017; 17:1293-302.

6. Woestenberg PJ, et al. Bivalent Vaccine Effectiveness Against Type-Specific HPV Positivity: Evidence for Cross-Protection Against Oncogenic Types Among Dutch STI Clinic Visitors. J Infect Dis 2018; 217:213-22.

7. Mesher $D$, et al. Continuing reductions in HPV $16 / 18$ in a population with high coverage of bivalent HPV vaccination in England: an ongoing crosssectional study. BMJ Open 2016; 6:e009915.

8. Woestenberg PJ, et al. No evidence for crossprotection of the HPV-16/18 vaccine against HPV6/11 positivity in female STI clinic visitors. J Infect 2017; 74:393-400.
9. Van Doorslaer K, et al. The Papillomavirus Episteme: a major update to the papillomavirus sequence database. Nucleic Acids Res 2017; 45:D499-D506.

10. Minh $B Q$, et al. Ultrafast approximation for phylogenetic bootstrap. Mol Biol Evol 2013; 30:118895.

11. Donken R, et al. High Effectiveness of the Bivalent Human Papillomavirus (HPV) Vaccine Against Incident and Persistent HPV Infections up to 6 Years After Vaccination in Young Dutch Women. J Infect Dis 2018; 217:1579-89.

12. Cuschieri K, et al. Impact of partial bivalent HPV vaccination on vaccine-type infection: a populationbased analysis. Br J Cancer 2016; 114:1261-4.

13. Didierlaurent AM, et al. ASO4, an aluminum saltand TLR4 agonist-based adjuvant system, induces a transient localized innate immune response leading to enhanced adaptive immunity. J Immunol 2009; 183:6186-97.

14. van der Weele $P$, et al. Effect of the bivalent HPV vaccine on viral load of vaccine and non-vaccine HPV types in incident clearing and persistent infections in young Dutch females. PLoS One 2019; 14:e0212927. 15. Gray P, et al. Evaluation of HPV type-replacement in unvaccinated and vaccinated adolescent femalesPost-hoc analysis of a community-randomized clinical trial (II). Int J Cancer 2018; 142:2491-500. 


\section{Supplementary information to Chapter 3}

Supplementary Table 3.1. Bivalent HPV VE against pooled estimates.

\begin{tabular}{|c|c|c|c|c|}
\hline & \multirow[t]{2}{*}{$\mathbf{N}(\%)$} & \multicolumn{3}{|c|}{ VE $(95 \% \mathrm{Cl})^{\mathrm{a}}$} \\
\hline & & HPV16/18 & $\begin{array}{l}\text { (possibly) oncogenic } \\
\text { types }^{b}\end{array}$ & $\begin{array}{l}\text { non-oncogenic } \\
\text { types }^{c}\end{array}$ \\
\hline \multicolumn{5}{|l|}{ All women } \\
\hline Unvaccinated & $799(38.0)$ & \multirow{2}{*}{$91.0(86.0-94.2)$} & \multirow{2}{*}{$25.8(17.7-33.2)$} & \multirow{2}{*}{$-4.9(-20.7-8.8)$} \\
\hline Vaccinated ( $\geq 1$ dose) & 1305 (62.0) & & & \\
\hline \multicolumn{5}{|c|}{ Women offered vaccination $<5$ years ago ${ }^{d}$} \\
\hline Unvaccinated & $185(42.5)$ & \multirow{2}{*}{$85.4(60.9-94.5)$} & \multirow{2}{*}{$20.6(-2.3-38.4)$} & \multirow{2}{*}{$5.9(-31.3-32.6)$} \\
\hline Vaccinated ( $\geq 1$ dose) & $250(57.5)$ & & & \\
\hline \multicolumn{5}{|c|}{ Women offered vaccination $5 / 6$ years ago } \\
\hline Unvaccinated & $306(37.7)$ & \multirow{2}{*}{92.1 (83.9 - 96.2) } & \multirow{2}{*}{$30.6(18.3-41.3)$} & \multirow{2}{*}{$-14.2(-42.8-8.6)$} \\
\hline Vaccinated ( $\geq 1$ dose) & $505(62.3)$ & & & \\
\hline \multicolumn{5}{|c|}{ Women offered vaccination $7 / 8$ years ago } \\
\hline Unvaccinated & $308(35.9)$ & \multirow{2}{*}{$91.7(83.4-95.8)$} & \multirow{2}{*}{$24.5(11.4-35.7)$} & \multirow{2}{*}{$-0.4(-24.4-18.9)$} \\
\hline Vaccinated ( $\geq 1$ dose) & $550(64.1)$ & & & \\
\hline \multicolumn{5}{|c|}{$\begin{array}{l}\text { a. VE was corrected for: age, migration background, education level, no. of sex partners in past } 6 \text { months, no. of lifetime sex } \\
\text { partners, age at sexual debut, history of any STI, history of hormonal contraceptives use, STI-related symptoms, and age } \\
\text { vaccination was offered. } \\
\text { b. Including HPV types HPV16/18/31/33/35/39/45/51/52/56/58/59/66/68 (73/97). } \\
\text { c. Including HPV types HPV6/11/34/40/42/43/44/53/54/70/74. } \\
\text { d. Time since vaccination offered was calculated from the date of sampling relative to the scheduled date of vaccination, } \\
\text { according to year of birth and the official schedule of HPV immunization in the Netherlands. For the catch-up cohorts, } \\
\text { vaccination was offered on } 1 \text { March } 2009 \text {. For the cohorts vaccinated routinely in the national immunization program, } \\
\text { vaccination was offered on } 1 \text { March in the year they turn } 13 \text { years old. }\end{array}$} \\
\hline
\end{tabular}


A) (Possibly) oncogenic types

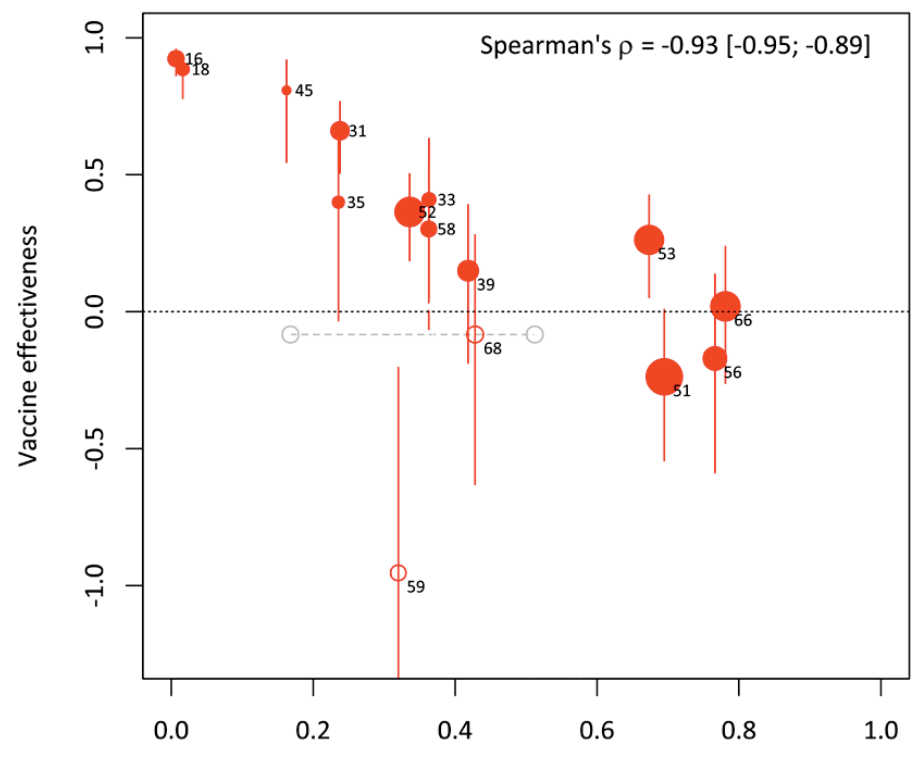

Phylogenetic distance to L1 VLP (protein analysis)

\section{B) Non-oncogenic types}

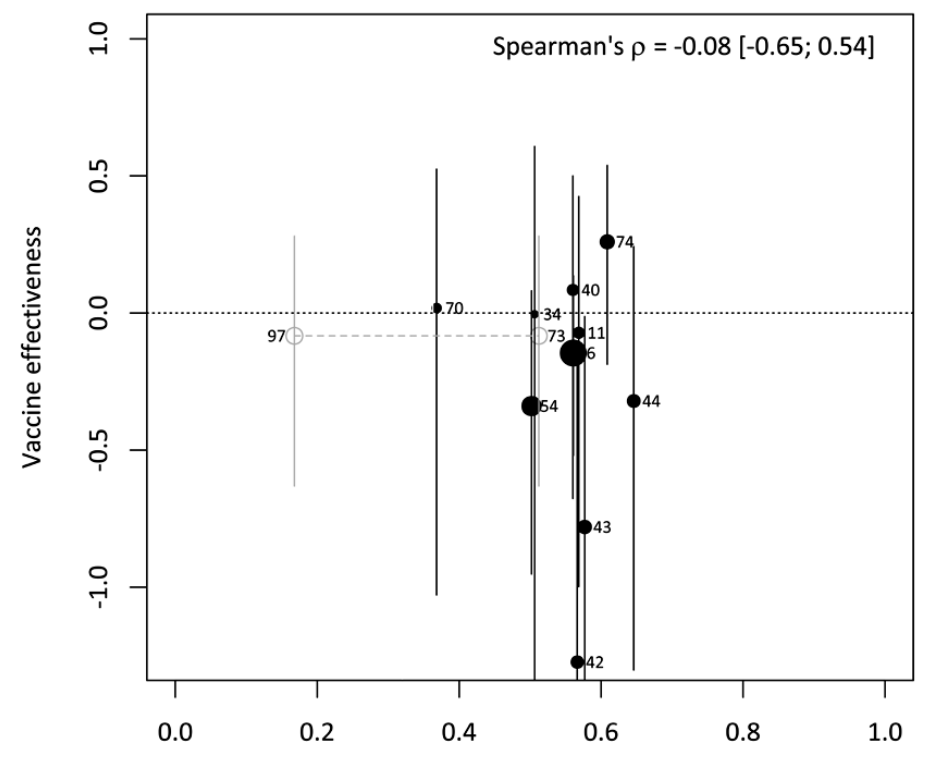

Phylogenetic distance to L1 VLP (protein analysis)

Supplementary Figure 3.1. Bivalent HPV VE versus phylogenetic distance to L1 VLP, by oncogenicity.

Note: Data points in A) and B) are similar to those in Figure 3.1A but include $95 \%$ Cls for VE. Spearman's rank correlation (with $95 \%$ confidence limits in brackets) in A) applies to (possibly) oncogenic types, excluding HPV59 and 68, whereas the correlation in B) applies to non-oncogenic types, excluding HPV73 and 97 . The correlation in A) was $\rho=-0.88(95 \% \mathrm{Cl}:-0.93 ;-0.78)$ when also excluding HPV16 and 18 (i.e. for non-vaccine types only). 


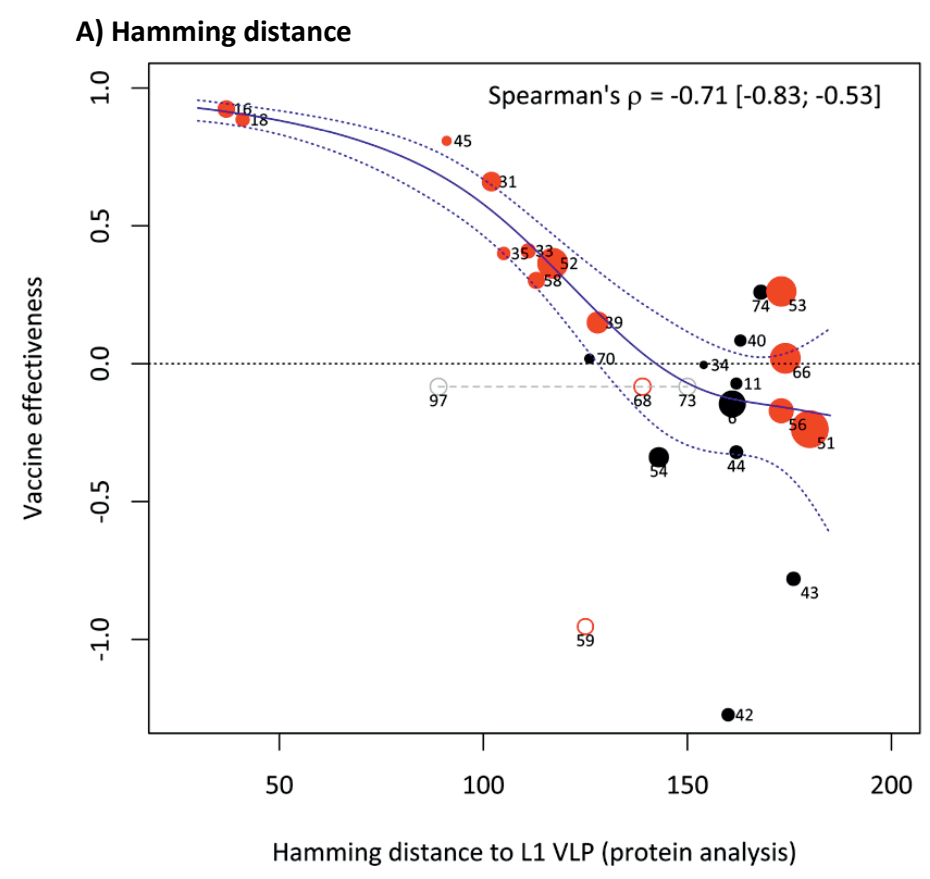

\section{B) L1 capsid genes}

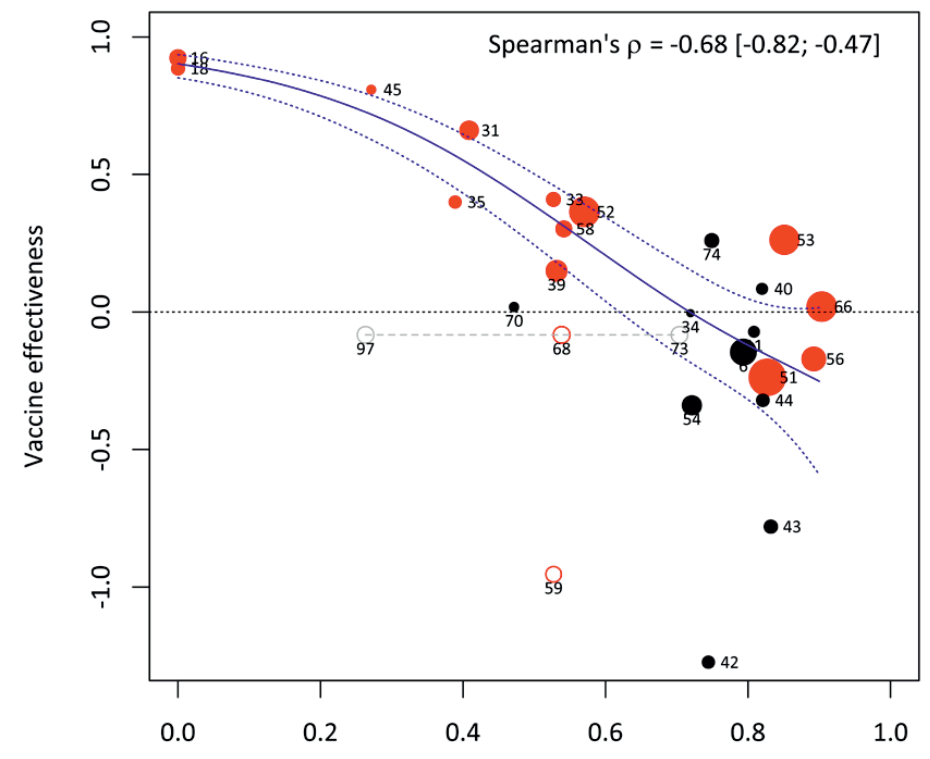

L1 phylogenetic distance to HPV-16 or 18 (DNA analysis) 


\section{C) Whole genome sequences}

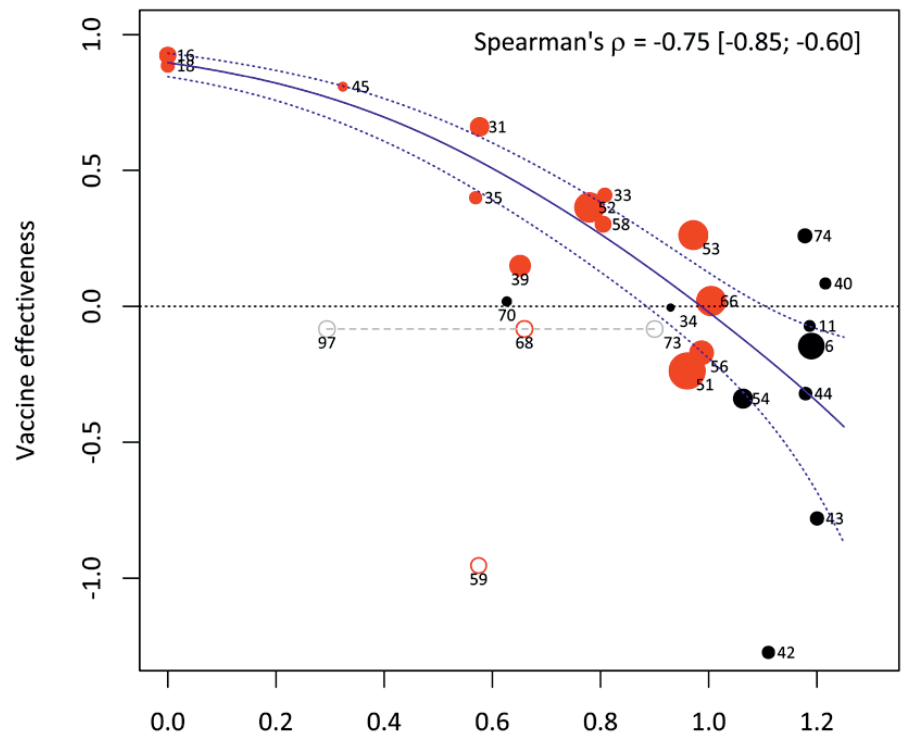

WGS phylogenetic distance to HPV-16 or 18 (DNA analysis)

\section{Supplementary Figure 3.2. Bivalent HPV VE as a function of alternative distance measures from HPV16} and 18.

Note: In all panels, the size of each data point is plotted proportional to the number of positive test results $n$ used in VE estimation, and Spearman's rank correlations (with $95 \%$ confidence limits in brackets) were calculated from all data points weighted by $\sqrt{ } \mathrm{n}$, excluding HPV59 and 68/73/97 (open circles). Red data points denote (possibly) oncogenic types in the SPF10-LiPA25 assay (Table 3.1), with HPV68 being indistinguishable from HPV73 and 97 (in grey). Hamming distance in A) expresses the number of positions at which the L1 proteins of reference types have different amino acids than the VLPs in 2vHPV. Phylogenetic distance on L1 capsid genes in B) was calculated from reference DNA sequences, using the transversion model (AG=CT) and unequal base frequencies counted from the data [9]. Substitution rate heterogeneity among alignment sites was incorporated by assuming Gammadistributed rates plus a fraction of invariable sites. Phylogenetic distance on whole genome sequences (WGS) in C) was calculated from reference DNA sequences, using the transversion model with $A G=C T$ and empirical base frequencies [9]. Substitution rate heterogeneity among alignment sites was incorporated via a free rate model with 5 categories. The estimated spline functions (in blue) are shown with $95 \%$ credible intervals (dotted lines). 
Supplementary Table 3.2. Rank correlation analysis between bivalent HPV VE and various distance measures.

\begin{tabular}{|c|c|c|c|c|c|}
\hline $\begin{array}{l}\text { Correlation } \\
\text { coefficient }\end{array}$ & Measure & Hamming ${ }^{a}$ & $\mathbf{A A}^{\mathrm{b}}$ & $L 1^{c}$ & $W_{G S}^{d}$ \\
\hline \multirow[t]{5}{*}{ Spearman's $\rho^{e}$} & VE & -0.713 & -0.703 & -0.680 & -0.753 \\
\hline & & $(p<.001)$ & $(p<.001)$ & $(p<.001)$ & $(p<.001)$ \\
\hline & Hamming \pm & & 0.963 & 0.957 & 0.744 \\
\hline & AA & & & 0.959 & 0.712 \\
\hline & L1 & & & & 0.765 \\
\hline \multirow[t]{5}{*}{ Spearman's $\rho_{\text {partial }}{ }^{f}$} & VE & -0.158 & -0.290 & 0.320 & -0.512 \\
\hline & & $(p=0.50)$ & $(p=0.20)$ & $(p=0.15)$ & $(p=0.012)$ \\
\hline & Hamming & & 0.429 & 0.414 & 0.046 \\
\hline & AA & & & 0.571 & -0.324 \\
\hline & $L 1$ & & & & 0.478 \\
\hline \multirow[t]{4}{*}{ Spearman's $\rho_{\text {partial }}$} & VE & (omitted) & -0.401 & 0.284 & -0.526 \\
\hline & & & $(p=0.063)$ & $(p=0.21)$ & $(p=0.0087)$ \\
\hline & AA & & & 0.910 & -0.337 \\
\hline & $L 1$ & & & & 0.546 \\
\hline
\end{tabular}

a. Minimum Hamming distance between aligned L1 AA sequences of reference types and virus-like particles in $2 \mathrm{vHPV}$ vaccine. b. Minimum phylogenetic distance between aligned L1 AA sequences of reference types and 2vHPV vaccine types, HPV16 and 18.

c. Minimum phylogenetic distance between aligned DNA sequences on the L1 capsid gene of reference types and $2 \mathrm{vHPV}$ vaccine types, HPV16 and 18.

d. Minimum phylogenetic distance between aligned DNA sequences on the whole viral genome of reference types and 2vHPV vaccine types, HPV16 and 18.

e. All correlation coefficients are weighted for the number of positive test results used in VE estimation (see Table 3.1).

f. Partial correlation coefficients are obtained from the inverse weighted covariance matrix. 



\section{Chapter 4}

\section{Bivalent vaccine effectiveness against anal human papillomavirus positivity among female sexually transmitted infection clinic visitors in the Netherlands}

Petra J. Woestenberg

Audrey J. King

Birgit H. B. van Benthem

Suzan Leussink

Marianne A. B. van der Sande

Christian J. P. A. Hoebe

Johannes A. Bogaards

The contents of this chapter have been published in The Journal of Infectious Diseases 


\section{Abstract}

Human papillomavirus (HPV) vaccines are indicated for anal cancer prevention, but evidence for vaccine effectiveness (VE) against anal HPV infections among women is limited. We estimated the VE ( $\geq 1$ dose) against anal HPV positivity of the bivalent vaccine, whose target types HPV16/18 are associated with approximately $90 \%$ of HPV-related anal cancers. Among 548 female sexually transmitted infection (STI) clinic visitors 16-24 years old who provided an anal swab sample as part of a repeated cross-sectional survey, VE against HPV16/18 was 89.9\% (95\% confidence interval, 63.0\%-97.2\%). Type-specific VE correlated well with VE against cervicovaginal HPV (Spearman's $\rho=0.76$ ), suggesting comparable effectiveness of HPV16/18 vaccination against genital and anal infections. 


\section{Introduction}

The sexually transmitted human papillomavirus (HPV) plays a causal role in the development of cervical, vaginal, vulvar, penile, oropharyngeal, and anal cancers. Anal cancer is more prevalent among women than among men and is responsible for the second largest HPV-related disease burden among women after cervical cancer [1]. The share of anal cancer in the total HPV-related disease burden is steadily increasing in many countries, owing to rising incidence trends and a current lack of effective screening opportunities [2]. Worldwide, over 20,000 women are affected by anal cancer each year, of which approximately 88\% are caused by oncogenic HPV infections, mainly HPV16/18 [1].

HPV vaccines hold promise for anal cancer control. However, though the high vaccine effectiveness (VE) of prophylactic HPV vaccines against cervicovaginal HPV infections has been widely documented in both randomized controlled trials (RCTs) and post-marketing studies, data on VE against anal HPV infections are scarce, especially among women. In 1 $\mathrm{RCT}$ of the bivalent (2vHPV) vaccine, post-hoc analyses demonstrated strong protection against anal positivity with vaccine types HPV16/18 (vaccine efficacy, $83.6 \%$ in the perprotocol population) and significant cross-protection against HPV31 and HPV45 [3].

In 2 other RCTs of the quadrivalent (4VHPV) vaccine, vaccine efficacy against anal HPV infections was estimated among men. In the per-protocol populations, vaccination afforded strong protection against anal persistent HPV16/18 infection (vaccine efficacy, >95\%) $[4,5]$. In addition, efficacy against high-grade anal intraepithelial neoplasia was demonstrated among men who have sex with men (vaccine efficacy, 74.9\%) [4]. Based on the latter study, the HPV vaccines received an indication for the prevention of anal cancer. One observational study of the 4VHPV vaccine explored the association between vaccination and anal HPV positivity among high-risk women and found a $64 \%$ reduction in the detection of anal HPV6/11/16/18 infections [6]. So far, post-marketing evidence on protection against anal HPV infection by the $2 \mathrm{HPV}$ vaccine has not become available.

The Netherlands has consistently used the 2vHPV vaccine (Cervarix; GSK) in the national immunization program (NIP). Largescale vaccination started in 2009 with a catch-up campaign for girls born in 1993-1996. Routine HPV vaccination was introduced in 2010 for girls in the year they turn 13 years old. We previously reported on type-specific VE of the 2vHPV vaccine against cervicovaginal HPV positivity [7]. In the current study, we evaluated VE against anal HPV positivity among female visitors to sexually transmitted infection (STI) clinics in the Netherlands. 


\section{Methods}

We used the same methods to estimate the VE against anal HPV positivity as previously used to estimate VE against cervicovaginal HPV positivity [7]. In short, we used data from the papillomavirus surveillance among STI clinic youngsters in the Netherlands (PASSYON) study. In this biennial cross-sectional study that started in 2009, 16- to 24-year-old visitors to STI clinics in the Netherlands were asked to provide a self-collected genital swab sample and to fill-in a questionnaire, including self-reported HPV vaccination status. A random subset of women were also asked to provide a self-collected anal swab sample on a voluntary basis. Because of financial constraints, not all women were asked for an anal swab sample; in the PASSYON study years 2015 and 2017, we aimed to obtain anal swab samples from a convenience sample of about $30 \%$ of the women, irrespective of self-reported vaccination status or sexual risk behavior.

Women who agreed to provide an anal swab sample were instructed to insert a swab about $3 \mathrm{~cm}$ into the anus and circle it around for 5-10 seconds. Swab samples were tested using the SPF10-DEIA-LiPA25 assay (DDL Diagnostics Laboratory). This broad-spectrum polymerase chain reaction assay can detect DNA of several HPV types, including the highrisk HPV (hrHPV) types 16/18/31/33/35/39/45/51/52/56/58/59 and the low-risk HPV (IrHPV) types HPV6/11. All participants provided informed consent.

For the current research question, we included women who had been eligible for HPV vaccination in the Netherlands (i.e. women born since 1993), reported their vaccination status, and provided an anal swab sample. We included data from the PASSYON study years 2011-2017.

Anal HPV positivity was compared between women who reported being vaccinated $(\geq 1$ dose) and those who reported not being vaccinated. To estimate the VE against anal HPV positivity, we used logistic mixed models with a random intercept, incorporating all clinically relevant HPV types (hrHPV and HPV6/11). Outcomes were type-specific HPV positivity, positivity for the vaccine types (HPV16/18, pooled), for the HPV types included in the nonavalent (9v) vaccine (HPV6/11/16/18/31/33/45/52/58, pooled), and for all acknowledged hrHPV types (HPV16/18/31/33/35/39/45/51/52/56/58/59, pooled). The pooled estimates were obtained as weighted averages of type-specific estimates. The analyses were adjusted for age and the variables that were found with $\chi^{2}$ tests to be associated with vaccination status (at a $p<.1$ level). VE was estimated as ( 1 - adjusted odds ratio) $\times 100 \%$.

Next, we assessed the Spearman's correlation between the type-specific anal and cervicovaginal VE estimates. The VE against cervicovaginal HPV positivity was estimated using the same study population and method used for estimating VE against anal HPV positivity. 
As sensitivity analyses, we estimated the VE against anal HPV positivity restricted to women who reported any history of anal sex (i.e., those considered at increased risk for anal cancer [8]) or restricted to women who were offered vaccination $\geq 5$ years ago, comparable to previous analyses [7]. All analyses were performed using SAS version 9.4 (SAS Institute Inc., Cary, NC), with a significance level of $p<.05$. Records with missing data were excluded from the analyses, because these represented $<5 \%$ of the study population.

\section{Results}

In the PASSYON study (years 2011-2017), a total of 2,413 women were eligible for HPV vaccination in the Dutch NIP, of whom 2,246 reported their vaccination status. Of these women, 548 (24\%) provided an anal swab sample (Supplementary Figure 4.1). Demographics and sexual risk behavior differed between women who provided an anal swab sample and those who did not, possibly related to the willingness of women to collect the sample. For example, anal swab samples were available for $51 \%$ of the women who reported anal sex in the past 6 months, compared with 19\% of those who reported no history of anal sex. Whether or not women provided an anal swab sample also differed by vaccination status. For instance, among women who reported no history of anal sex, vaccinated women were more likely than unvaccinated women to provide a sample $(21 \%$ vs $15 \%$, respectively) (Supplementary Table 4.1 ).

Of the 548 women who did provide an anal swab and were included in the analyses, 357 (65\%) reported being vaccinated with $\geq 1$ dose (43 women reported incomplete dosing). Vaccinated women were more likely to report a high education level, no history of anal sex, more sex partners in the past 6 months, no STI-related symptoms, and a history of using hormonal contraceptive (Table 4.1). The VE measures were adjusted for all these variables.

Table 4.1. Characteristics of the study population used to estimate VE against anal HPV positivity.

\begin{tabular}{|c|c|c|c|c|}
\hline & $\begin{array}{l}\text { Total (N=548); } \\
\mathrm{N}(\%)\end{array}$ & $\begin{array}{l}\text { Unvaccinated }^{a} \\
\text { (n=191); } N(\%)\end{array}$ & $\begin{array}{l}\text { Vaccinated }^{a}(\geq 1 \\
\text { dose, } n=357) ; N(\%)\end{array}$ & $\begin{array}{l}p \text { value } \\
\left.\text { ( } \chi^{2} \text { test }\right)\end{array}$ \\
\hline Age & & & & 0.36 \\
\hline $16-18$ years & 72 (13.1) & $29(15.2)$ & $43(12.0)$ & \\
\hline $19-21$ years & $330(60.2)$ & $117(61.3)$ & $213(59.7)$ & \\
\hline $22-24$ years & $146(26.6)$ & $45(23.6)$ & $101(28.3)$ & \\
\hline Migration background & & & & 0.10 \\
\hline Native Dutch & $420(76.8)$ & $139(72.8)$ & 281 (78.9) & \\
\hline Not native Dutch & $127(23.2)$ & $52(27.2)$ & $75(21.1)$ & \\
\hline Education level $^{c}$ & & & & $<.01$ \\
\hline Low/middle & $115(21.0)$ & $55(28.8)$ & $60(16.8)$ & \\
\hline High & $433(79.0)$ & $136(71.2)$ & $297(83.2)$ & \\
\hline
\end{tabular}


Table 4.1. (Continued).

\begin{tabular}{|c|c|c|c|c|}
\hline & $\begin{array}{l}\text { Total }(\mathrm{N}=548) ; \\
\mathrm{N}(\%)\end{array}$ & $\begin{array}{l}\text { Unvaccinated }^{a} \\
\text { (n=191); } N(\%)\end{array}$ & $\begin{array}{l}\text { Vaccinated }^{a}(\geq 1 \\
\text { dose, } n=357) ; N \text { (\%) }\end{array}$ & $\begin{array}{l}\text { value } \\
\left.\text { ( } \chi^{2} \text { test }\right)\end{array}$ \\
\hline \multicolumn{4}{|l|}{ History of anal sex } & 0.02 \\
\hline No & $295(54.0)$ & 91 (47.9) & $204(57.3)$ & \\
\hline Yes, past 6 months & $142(26.0)$ & $63(33.2)$ & $79(22.2)$ & \\
\hline Yes, ever & $109(20.0)$ & $36(18.9)$ & $73(20.5)$ & \\
\hline \multicolumn{4}{|c|}{ No. of sex partners, past 6 months ${ }^{d}$} & 0.05 \\
\hline $0-1$ partners & $133(24.3)$ & $55(28.9)$ & $78(21.8)$ & \\
\hline 2-3 partners & $263(48.1)$ & 93 (48.9) & $170(47.6)$ & \\
\hline$\geq 4$ partners & $151(27.6)$ & $42(22.1)$ & $109(30.5)$ & \\
\hline \multicolumn{4}{|c|}{ No. of sex partners, lifetime ${ }^{d}$} & 0.18 \\
\hline$\leq 3$ partners & $110(20.4)$ & $46(24.7)$ & $64(18.1)$ & \\
\hline 4-6 partners & $142(26.3)$ & $48(25.8)$ & $94(26.6)$ & \\
\hline$\geq 7$ partners & $288(53.3)$ & $92(49.5)$ & $196(55.4)$ & \\
\hline \multicolumn{4}{|l|}{ Age at sexual debut ${ }^{d}$} & 0.96 \\
\hline$\leq 14$ years & $75(13.8)$ & $25(13.4)$ & $50(14.1)$ & \\
\hline $15-16$ years & $283(52.2)$ & 99 (52.9) & $184(51.8)$ & \\
\hline$\geq 17$ years & $184(33.9)$ & $63(33.7)$ & $121(34.1)$ & \\
\hline \multicolumn{4}{|l|}{ History of any STI } & 0.60 \\
\hline No & $291(53.1)$ & $97(50.8)$ & $194(54.3)$ & \\
\hline Yes & $155(28.3)$ & 59 (30.9) & $96(26.9)$ & \\
\hline Never tested & $102(18.6)$ & $35(18.3)$ & $67(18.8)$ & \\
\hline \multicolumn{4}{|c|}{ Current anal chlamydia/gonorrhoea infection } & 0.28 \\
\hline No & $173(31.6)$ & $68(35.6)$ & $105(29.4)$ & \\
\hline Yes & $32(5.8)$ & $12(6.3)$ & $20(5.6)$ & \\
\hline Not tested & $343(62.6)$ & $111(58.1)$ & $232(65.0)$ & \\
\hline \multicolumn{4}{|l|}{ Notified for STIs } & 0.59 \\
\hline No & $468(85.4)$ & $161(84.3)$ & $307(86.0)$ & \\
\hline Yes & $80(14.6)$ & $30(15.7)$ & $50(14.0)$ & \\
\hline \multicolumn{4}{|l|}{ STI-related symptoms } & 0.05 \\
\hline No & $411(75.1)$ & $134(70.2)$ & $277(77.8)$ & \\
\hline Yes & $136(24.9)$ & $57(29.8)$ & $79(22.2)$ & \\
\hline \multicolumn{4}{|c|}{ Condom use with casual partners, past 6 months ${ }^{e}$} & 0.24 \\
\hline Inconsistent & $254(46.7)$ & $83(43.9)$ & $171(48.2)$ & \\
\hline Consistent & $190(34.9)$ & $64(33.9)$ & $126(35.5)$ & \\
\hline No casual partners & $100(18.4)$ & $42(22.2)$ & $58(16.3)$ & \\
\hline \multicolumn{4}{|c|}{ History of using hormonal contraceptives } & $<.01$ \\
\hline No & $15(2.8)$ & $10(5.3)$ & $5(1.4)$ & \\
\hline Yes & $524(97.2)$ & $177(94.7)$ & $347(98.6)$ & \\
\hline
\end{tabular}

Totals vary because of missing values.

a. Categories based on self-reported vaccination status.

b. Based on (parental) country of birth. A women was defined native Dutch if both parents were born in the Netherlands.

c. High educational level included school of higher general secondary education, pre-university education, university of applied sciences, and university. Low/middle educational level included all other levels of education.

d. Vaginal or anal sex.

e. Inconsistent included reporting never, rarely and "sometimes I do, sometimes I do not" condom use. Consistent included reporting often or always condom use. 
Only 2 vaccinated women ( $0.6 \%$ ) tested positive for anal HPV16, and only 1 (0.3\%) for HPV18 (Supplementary Table 4.2). In comparison, $4.2 \%$ and $3.1 \%$ of unvaccinated women tested positive for anal HPV16 and HPV18, respectively, leading to an adjusted VE of $88.2 \%$ (95\% confidence interval [Cl], 41.3\%-97.6\%) against anal HPV16 and 91.9\% (95\% Cl, 30.5\%-99.1\%) against anal HPV18 (Figure 4.1). The VE against anal HPV16/18 combined was $89.9 \%$ (95\% $\mathrm{Cl}, 63.0 \%-97.2 \%)$. None of the vaccinated women were positive for anal HPV45, compared with $3.1 \%$ of the unvaccinated women (VE, $100 \%$; unadjusted $95 \% \mathrm{Cl}, 66.5 \%-100 \%$ ). We also observed cross-protection against anal HPV31 (VE, 73.0\%; 95\% Cl, 25.5\%-90.2\%). The typespecific VE against anal HPV positivity correlated well with the VE against cervicovaginal HPV positivity (Spearman's $\rho=0.76 ; p<.01$ ).

Of the total study population, 251 women (46\%) reported any history of anal sex. In this subgroup, the anal prevalence of an hrHPV type or HPV6/11 was higher than among women who reported no history of anal sex ( $41 \%$ vs $34 \%$ respectively), and the VE against anal HPV16/18 was 95.5\% (95\% Cl, 63.3\%-99.5\%) (Supplementary Figure 4.2). Most women ( $n=491[90 \%]$ ) were offered vaccination $\geq 5$ years ago (range, 5-8 years). In this subgroup, the VE against anal HPV16/18 was comparable to that in the total population, at $90.0 \%$ (95\% $\mathrm{Cl}, 63.3 \%-97.3 \%$ ) (Supplementary Figure 4.3).

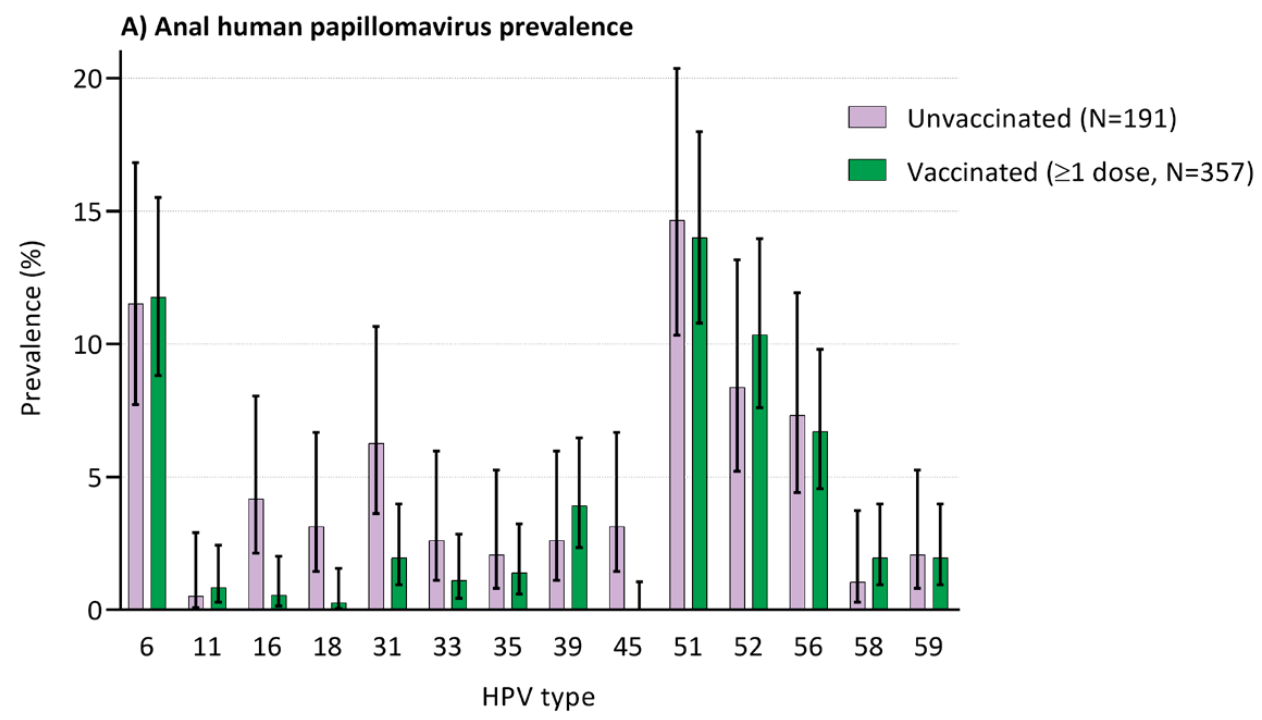


B) Vaccine effectiveness against anal HPV

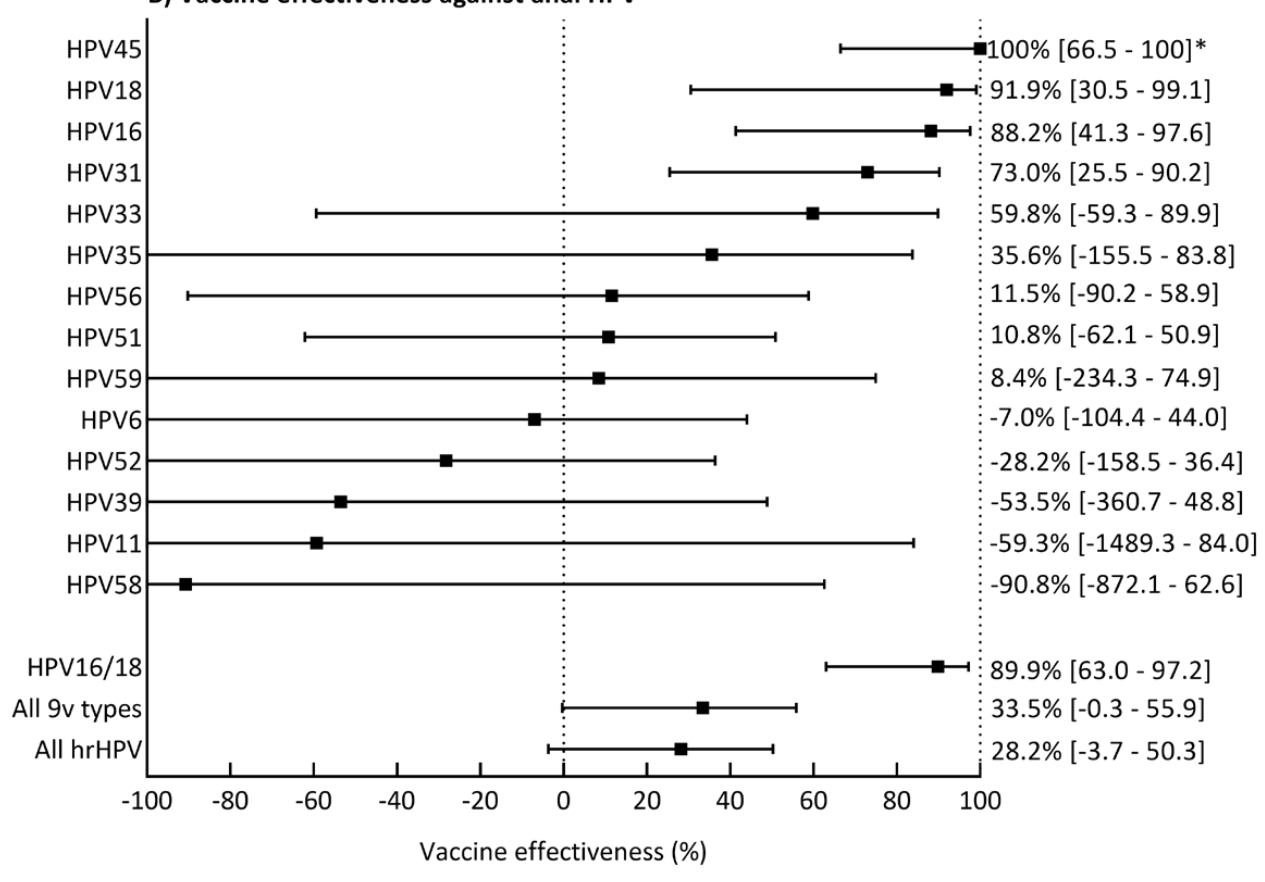

Figure 4.1. A) Anal HPV prevalence by self-reported vaccination status and B) adjusted VE against anal HPV for $\geq 1$ dose.

Note: All 9v types included all types of the 9vHPV vaccine: HPV6/11/16/18/31/33/45/52/58. All hrHPV types included HPV16/18/31/33/35/39/45/51/52/56/58/59. VE was estimated as ( 1 - adjusted odds ratio) $\times 100 \%$. Odds ratios were adjusted for age, education level, history of anal sex, number of sex partners in the past 6 months, STI-related symptoms, and history of hormonal contraceptives use. ${ }^{*}$ Unadjusted $95 \% \mathrm{Cl}$ based on score confidence limits for the odds ratio.

\section{Discussion}

We demonstrated high effectiveness of the 2vHPV vaccine against anal positivity with vaccine types HPV16/18 up to 8 years after vaccination. We also demonstrated crossprotection against anal HPV45 and HPV31 and a high correlation between anal and cervicovaginal VE. These results confirm that HPV vaccination protects against anal HPV infection among women in a population-based setting, thereby suggesting that the benefits of HPV vaccination will extend to anal cancer prevention.

To our knowledge, this is the first observational study reporting VE of the 2VHPV vaccine against anal HPV positivity. A strength of our study is the population-based design. A limitation is the differential sexual risk behavior between vaccinated and unvaccinated women who provided an anal swab sample, possibly related to differences in demographics between vaccinated and unvaccinated women in general, such as educational level and migration background [9]. We adjusted for known differences between vaccinated and 
unvaccinated women who did provide an anal swab sample, but we cannot rule out residual confounding. To mitigate unmeasured confounding, we used mixed models that allow for fixed effects of known risk factors as well as random effects in individual risk for HPV infection.

Another limitation is the self-reported vaccination status. However, we previously showed that self-reported vaccination status agreed excellently with HPV16 and HPV18 antibody levels, suggesting limited bias [7]. Moreover, misclassification according to selfreported vaccination status should lead to an underestimation of the effect of vaccination. In addition, the majority of our study population was eligible as part of the catch-up program (84\%), meaning that most vaccinated women were between 12 and 16 years old when offered vaccination. Some women in our study population might have acquired anal HPV infection before getting vaccinated, negatively affecting VE [3, 4]. Finally, although we had detailed information on sexual risk behavior, this was not specific for anal sex. For instance, the number of sex partners included anal sex as well as vaginal sex partners.

Our VE against anal HPV16/18 positivity was comparable to the vaccine efficacy reported against anal HPV16/18 infection in the RCTs, conducted in women for the 2vHPV vaccine and in men for the $4 \mathrm{VHPV}$ vaccine [3-5]. In the only other observational study, the VE against anal HPV6/11/16/18 positivity was somewhat lower (64\%), which could be related to the relatively high anal HPV prevalence before vaccination in that study [6]. Although few effectiveness measures against anal HPV infection are available, the limited data, including ours, suggest an equally high VE against anal as against genital HPV infection. We also observed cross-protection against anal HPV31 and HPV45, consistently observed cross-protective types in 2vHPV vaccine studies with regard to cervicovaginal HPV infections [7, 10-13]. Comparable cross-protection is further supported by the high correlation between type-specific VE against anal and cervicovaginal HPV infections in our study.

The mechanism of protection against anal HPV infection is unclear in the current study. Many women who reported no history of anal sex tested positive for anal HPV (34\%). This has also been reported elsewhere [14] and resembles anal chlamydia infection patterns [15]. In part, this could be due to underreporting of anal sex, but it is also possible that the relatively high positivity rate for anal HPV is partly explained by autoinoculation from genital HPV infection sites. In these cases, protection against anal HPV infection would be an indirect effect of vaccination, following from the prevention of genital HPV infection. However, the VE against anal HPV16/18 positivity was equally high (96\%) among women who did report a history of anal sex, suggesting undiminished effectiveness with regard to direct protection.

In conclusion, we demonstrated high VE, up to 8 years after vaccination, of the $2 \mathrm{vHPV}$ vaccine against anal HPV infections, which was comparable to prevention of genital 
infections. The VE was particularly high against anal HPV16/18 positivity. These findings are promising for anal cancer control, given that nearly $90 \%$ of all HPV-related anal cancers are associated with HPV16/18 [1]. With an increasing incidence of HPV-related anal cancer and a current lack of effective screening opportunities, HPV vaccination provides a tremendous opportunity for anal cancer prevention.

\section{References}

1. de Martel C, et al. Worldwide burden of cancer attributable to HPV by site, country and HPV type. Int J Cancer 2017; 141:664-70.

2. Kang YJ, et al. Anal cancer in high-income countries: Increasing burden of disease. PLoS One 2018; 13:e0205105.

3. Kreimer AR, et al. Efficacy of a bivalent HPV 16/18 vaccine against anal HPV 16/18 infection among young women: a nested analysis within the Costa Rica Vaccine Trial. Lancet Oncol 2011; 12:862-70.

4. Palefsky JM, et al. HPV vaccine against anal HPV infection and anal intraepithelial neoplasia. N Engl J Med 2011; 365:1576-85.

5. Mikamo $\mathrm{H}$, et al. Efficacy, safety, and immunogenicity of a quadrivalent HPV vaccine in Japanese men: A randomized, Phase 3, placebocontrolled study. Vaccine 2019; 37:1651-8.

6. Schlecht NF, et al. Risk of Delayed Human Papillomavirus Vaccination in Inner-City Adolescent Women. J Infect Dis 2016; 214:1952-60.

7. Woestenberg PJ, et al. Bivalent Vaccine Effectiveness Against Type-Specific HPV Positivity: Evidence for Cross-Protection Against Oncogenic Types Among Dutch STI Clinic Visitors. J Infect Dis 2018; 217:213-22.

8. Daling JR, et al. Human papillomavirus, smoking, and sexual practices in the etiology of anal cancer. Cancer 2004; 101:270-80.

9. Rondy $M$, et al. Determinants for HPV vaccine uptake in the Netherlands: A multilevel study. Vaccine 2010; 28:2070-5.
10. Kavanagh $\mathrm{K}$, et al. Changes in the prevalence of human papillomavirus following a national bivalent human papillomavirus vaccination programme in Scotland: a 7-year cross-sectional study. Lancet Infect Dis 2017; 17:1293-302.

11. Donken R, et al. High Effectiveness of the Bivalent Human Papillomavirus (HPV) Vaccine Against Incident and Persistent HPV Infections up to 6 Years After Vaccination in Young Dutch Women. J Infect Dis 2018; 217:1579-89.

12. Kudo R, et al. Bivalent Human Papillomavirus Vaccine Effectiveness in a Japanese Population: High Vaccine-Type-Specific Effectiveness and Evidence of Cross-Protection. J Infect Dis 2019; 219:382-90.

13. Skinner SR, et al. Human papillomavirus (HPV)16/18 AS04-adjuvanted vaccine for the prevention of cervical cancer and HPV-related diseases. Expert Rev Vaccines 2016; 15:367-87.

14. Castro FA, et al. Prevalence of and risk factors for anal human papillomavirus infection among young healthy women in Costa Rica. J Infect Dis 2012; 206:1103-10.

15. van Liere G, et al. High Proportion of Anorectal Chlamydia trachomatis and Neisseria gonorrhoeae After Routine Universal Urogenital and Anorectal Screening in Women Visiting the Sexually Transmitted Infection Clinic. Clin Infect Dis 2017; 64:1705-10. 


\section{Supplementary information to Chapter 4}

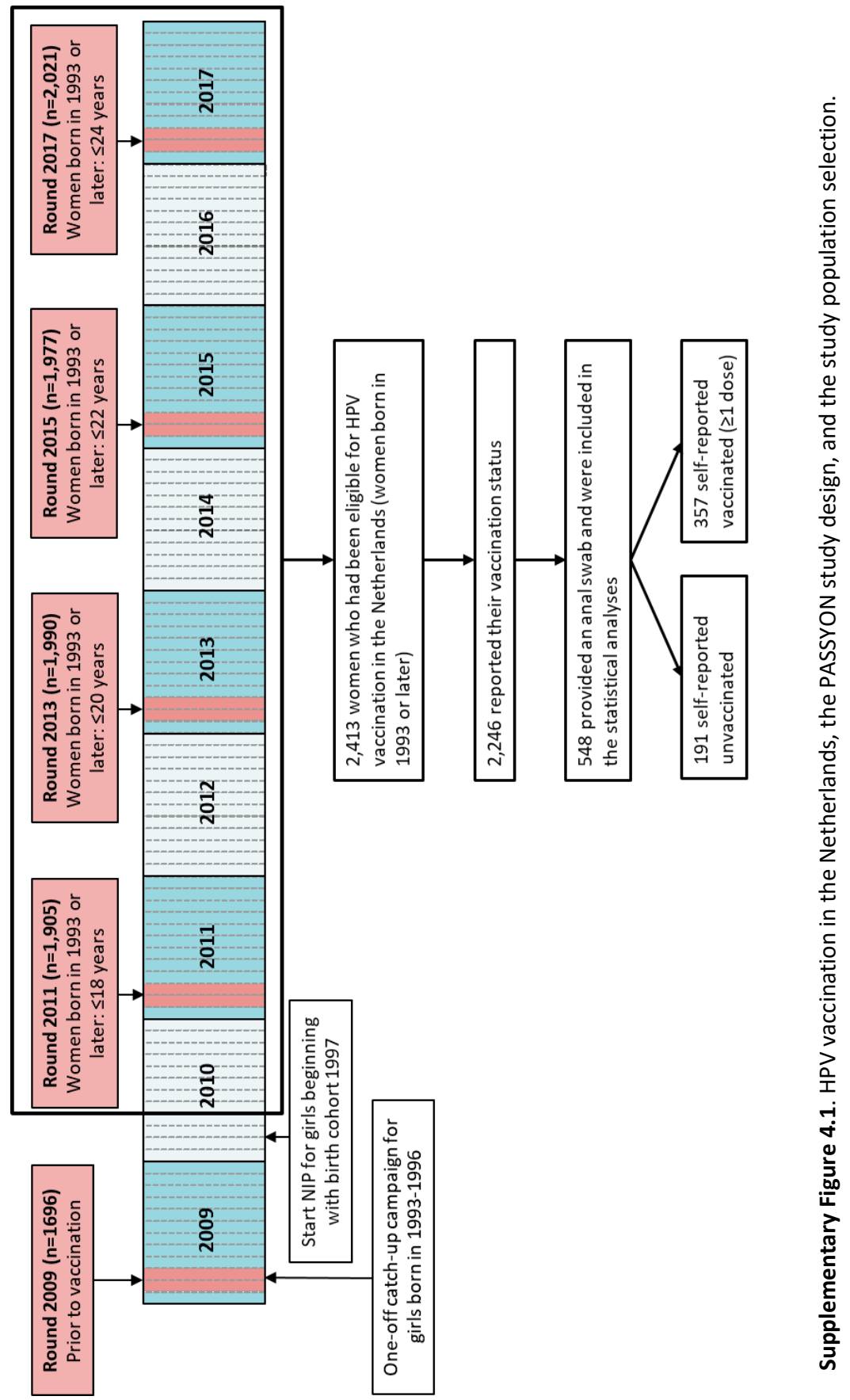


Supplementary Table 4.1. Characteristics of the women included in the PASSYON study who had been eligible for HPV vaccination in the Dutch NIP.

\begin{tabular}{|c|c|c|c|c|c|c|}
\hline & \multicolumn{2}{|c|}{ Total $(\mathrm{N}=2,246)$} & \multicolumn{2}{|c|}{$\begin{array}{l}\text { Unvaccinated }^{\mathrm{a}} \\
(\mathrm{N}=855)\end{array}$} & \multicolumn{2}{|c|}{$\begin{array}{l}\text { Vaccinated }^{\mathrm{a}}(\geq 1 \\
\text { dose, } \mathrm{N}=1,391)\end{array}$} \\
\hline & $\mathbf{N}$ & $\begin{array}{l}\text { Anal swab } \\
n(\%)^{b}\end{array}$ & $\mathbf{N}$ & $\begin{array}{l}\text { Anal swab } \\
n(\%)^{b}\end{array}$ & $\mathbf{N}$ & $\begin{array}{l}\text { Anal swab } \\
n(\%)^{b}\end{array}$ \\
\hline \multicolumn{7}{|l|}{ Age } \\
\hline $16-18$ years & 440 & $72(16.4)$ & 176 & $29(16.5)$ & 264 & $43(16.3)$ \\
\hline 19-21 years & 1335 & $330(24.7)$ & 512 & $117(22.9)$ & 823 & $213(25.9)$ \\
\hline 22-24 years & 471 & $146(31.0)$ & 167 & 45 (26.9) & 304 & $101(33.2)$ \\
\hline \multicolumn{7}{|c|}{ Migration background ${ }^{\mathrm{C}}$} \\
\hline Native Dutch & 1764 & $420(23.8)$ & 627 & $139(22.2)$ & 1137 & $281(24.7)$ \\
\hline Not native Dutch & 465 & $127(27.3)$ & 221 & $52(23.5)$ & 244 & 75 (30.7) \\
\hline \multicolumn{7}{|l|}{ Education level $^{d}$} \\
\hline Low/middle & 609 & $115(18.9)$ & 294 & $55(18.7)$ & 315 & $60(19.0)$ \\
\hline High & 1636 & $433(26.5)$ & 561 & $136(24.2)$ & 1075 & $297(27.6)$ \\
\hline \multicolumn{7}{|l|}{ History of anal sex } \\
\hline No & 1582 & $295(18.6)$ & 593 & $91(15.3)$ & 989 & $204(20.6)$ \\
\hline Yes, past 6 months & 280 & $142(50.7)$ & 118 & $63(53.4)$ & 162 & $79(48.8)$ \\
\hline Yes, ever & 369 & $109(29.5)$ & 137 & $36(26.3)$ & 232 & $73(31.5)$ \\
\hline \multicolumn{7}{|c|}{ No. of sex partners, past 6 months ${ }^{e}$} \\
\hline $0-1$ partners & 600 & $133(22.2)$ & 265 & $55(20.8)$ & 335 & $78(23.3)$ \\
\hline 2-3 partners & 1084 & $263(24.3)$ & 394 & $93(23.6)$ & 690 & $170(24.6)$ \\
\hline$\geq 4$ partners & 561 & $151(26.9)$ & 195 & $42(21.5)$ & 366 & $109(29.8)$ \\
\hline \multicolumn{7}{|c|}{ No. of sex partners, lifetime $e^{e}$} \\
\hline$\leq 3$ partners & 502 & $110(21.9)$ & 215 & $46(21.4)$ & 287 & $64(22.3)$ \\
\hline 4-6 partners & 645 & $142(22.0)$ & 244 & $48(19.7)$ & 401 & $94(23.4)$ \\
\hline$\geq 7$ partners & 1071 & $288(26.9)$ & 383 & $92(24.0)$ & 688 & $196(28.5)$ \\
\hline \multicolumn{7}{|l|}{ Age at sexual debute } \\
\hline$\leq 14$ years & 336 & $75(22.3)$ & 146 & $25(17.1)$ & 190 & $50(26.3)$ \\
\hline $15-16$ years & 1121 & $283(25.2)$ & 418 & $99(23.7)$ & 703 & $184(26.2)$ \\
\hline$\geq 17$ years & 762 & $184(24.1)$ & 278 & $63(22.7)$ & 484 & $121(25.0)$ \\
\hline \multicolumn{7}{|l|}{ History of any STI } \\
\hline No & 1178 & $291(24.7)$ & 421 & $97(23.0)$ & 757 & $194(25.6)$ \\
\hline Yes & 579 & $155(26.8)$ & 241 & $59(24.5)$ & 338 & $96(28.4)$ \\
\hline Never tested & 482 & $102(21.2)$ & 191 & $35(18.3)$ & 291 & $67(23.0)$ \\
\hline \multicolumn{7}{|c|}{ Current anal chlamydia/gonorrhoea infection } \\
\hline No & 312 & $173(55.4)$ & 122 & $68(55.7)$ & 190 & $105(55.3)$ \\
\hline Yes & 71 & $32(45.1)$ & 26 & $12(46.2)$ & 45 & $20(44.4)$ \\
\hline Not tested & 1863 & $343(18.4)$ & 707 & $111(15.7)$ & 1156 & $232(20.1)$ \\
\hline \multicolumn{7}{|l|}{ Notified for STIs } \\
\hline No & 1913 & $468(24.5)$ & 721 & $161(22.3)$ & 1192 & $307(25.8)$ \\
\hline Yes & 312 & $80(25.6)$ & 125 & $30(24.0)$ & 187 & $50(26.7)$ \\
\hline \multicolumn{7}{|l|}{ STI-related symptoms } \\
\hline No & 1666 & $411(24.7)$ & 601 & $134(22.3)$ & 1065 & $277(26.0)$ \\
\hline Yes & 558 & $136(24.4)$ & 245 & $57(23.3)$ & 313 & $79(25.2)$ \\
\hline
\end{tabular}


Supplementary Table 4.1. (Continued).

\begin{tabular}{|c|c|c|c|c|c|c|}
\hline & \multicolumn{2}{|c|}{ Total $(\mathrm{N}=\mathbf{2 , 2 4 6 )}$} & \multicolumn{2}{|c|}{$\begin{array}{l}\text { Unvaccinated }^{a} \\
\qquad(\mathrm{~N}=855)\end{array}$} & \multicolumn{2}{|c|}{$\begin{array}{l}\text { Vaccinated }^{\mathrm{a}}(\geq 1 \\
\text { dose, } \mathrm{N}=1,391)\end{array}$} \\
\hline & $\mathbf{N}$ & $\begin{array}{l}\text { Anal swab } \\
n(\%)^{b}\end{array}$ & $\mathbf{N}$ & $\begin{array}{l}\text { Anal swab } \\
\text { n (\%) }\end{array}$ & $\mathbf{N}$ & $\begin{array}{l}\text { Anal swab } \\
n(\%)^{b}\end{array}$ \\
\hline \multicolumn{7}{|c|}{ Condom use with casual partners, past 6 months $^{f}$} \\
\hline Inconsistent & 1088 & $254(23.3)$ & 405 & $83(20.5)$ & 683 & $171(25.0)$ \\
\hline Consistent & 696 & $190(27.3)$ & 253 & $64(25.3)$ & 443 & $126(28.4)$ \\
\hline No casual partners & 450 & $100(22.2)$ & 191 & $42(22.0)$ & 259 & $58(22.4)$ \\
\hline \multicolumn{7}{|c|}{ History of using hormonal contraceptives } \\
\hline No & 74 & $15(20.3)$ & 43 & $10(23.3)$ & 31 & $5(16.1)$ \\
\hline Yes & 2135 & $524(24.5)$ & 798 & $177(22.2)$ & 1337 & $347(26.0)$ \\
\hline \multicolumn{7}{|c|}{$\begin{array}{l}\text { Totals vary because of missing values. } \\
\text { a. Based on self-reported vaccination status. } \\
\text { b. Percentage who provided an anal swab. } \\
\text { c. Based on (parental) country of birth. A women was defined native Dutch if both parents were born in the Netherlands. } \\
\text { d. High educational level included school of higher general secondary education, pre-university education, university of applied } \\
\text { sciences, and university. Low/middle educational level included all other levels of education. } \\
\text { e. Vaginal or anal sex. } \\
\text { f. Inconsistent included reporting never, rarely and "sometimes I do, sometimes I do not" condom use. Consistent included } \\
\text { reporting often or always condom use. }\end{array}$} \\
\hline
\end{tabular}

Supplementary Table 4.2. Anal HPV prevalence by vaccination status.

\begin{tabular}{|c|c|c|c|c|}
\hline & \multicolumn{2}{|c|}{ Unvaccinated $^{\mathrm{a}}(\mathrm{N}=191)$} & \multicolumn{2}{|c|}{ Vaccinated $^{\mathrm{a}}(\geq 1$ dose, $\mathrm{N}=357$ ) } \\
\hline & n positive & \% positive (95\% Cl) & n positive & \% positive $(95 \% \mathrm{Cl})$ \\
\hline HPV6 & 22 & $11.5(7.7-16.8)$ & 42 & $11.8(8.8-15.5)$ \\
\hline HPV11 & 1 & $0.5(0.1-2.9)$ & 3 & $0.8(0.3-2.4)$ \\
\hline HPV16 & 8 & $4.2(2.1-8.0)$ & 2 & $0.6(0.2-2.0)$ \\
\hline HPV18 & 6 & $3.1(1.4-6.7)$ & 1 & $0.3(0.0-1.6)$ \\
\hline HPV31 & 12 & $6.3(3.6-10.7)$ & 7 & $2.0(1.0-4.0)$ \\
\hline HPV33 & 5 & $2.6(1.1-6.0)$ & 4 & $1.1(0.4-2.8)$ \\
\hline HPV35 & 4 & $2.1(0.8-5.3)$ & 5 & $1.4(0.6-3.2)$ \\
\hline HPV39 & 5 & $2.6(1.1-6.0)$ & 14 & $3.9(2.4-6.5)$ \\
\hline HPV45 & 6 & $3.1(1.4-6.7)$ & 0 & $0.0(0.0-1.1)$ \\
\hline HPV51 & 28 & $14.7(10.3-20.4)$ & 50 & $14.0(10.8-18.0)$ \\
\hline HPV52 & 16 & $8.4(5.2-13.2)$ & 37 & $10.4(7.6-14.0)$ \\
\hline HPV56 & 14 & 7.3 (4.4 - 11.9) & 24 & $6.7(4.6-9.8)$ \\
\hline HPV58 & 2 & $1.0(0.3-3.7)$ & 7 & $2.0(1.0-4.0)$ \\
\hline HPV59 & 4 & $2.1(0.8-5.3)$ & 7 & $2.0(1.0-4.0)$ \\
\hline
\end{tabular}

a. Based on self-reported vaccination status. 


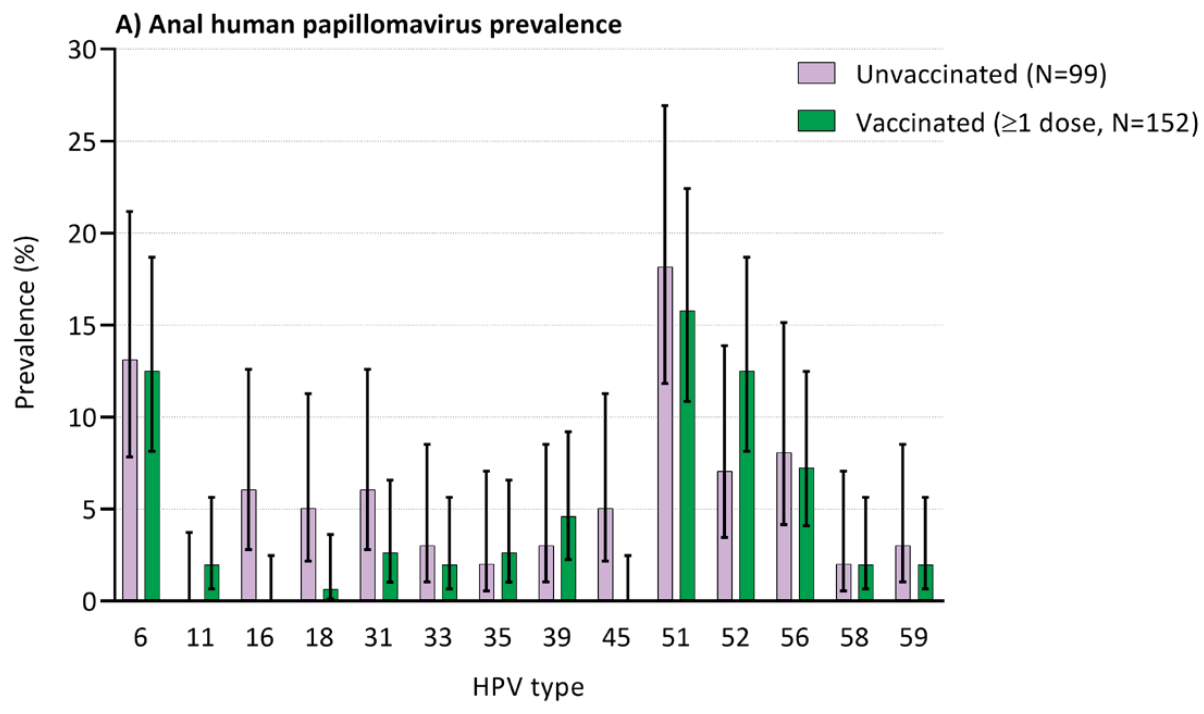

B) Vaccine effectiveness against anal HPV

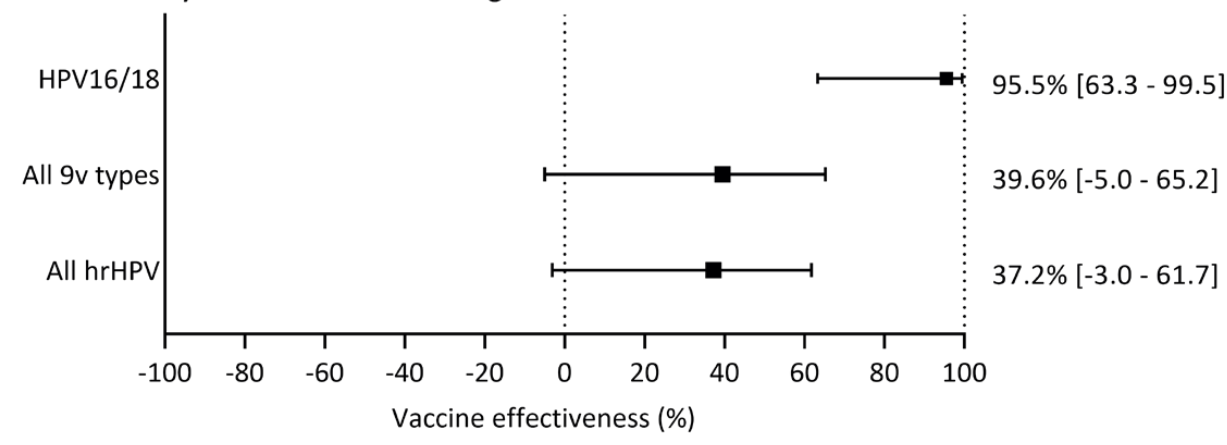

Supplementary Figure 4.2. Anal HPV prevalence and adjusted VE, restricted to women who reported any history of anal sex. A) Anal HPV prevalence by self-reported vaccination status and B) adjusted VE against anal HPV for at least one dose.

Note: All 9v types included all types of the 9vHPV vaccine: HPV6/11/16/18/31/33/45/52/58. All hrHPV types included: HPV16/18/31/33/35/39/45/51/52/56/58/59. VE was estimated as (1- odds ratio)*100\% and was adjusted for age, education level, anal sex in the past 6 months, number of sex partners in the past 6 months, STI-related symptoms, and history of hormonal contraceptives use. 


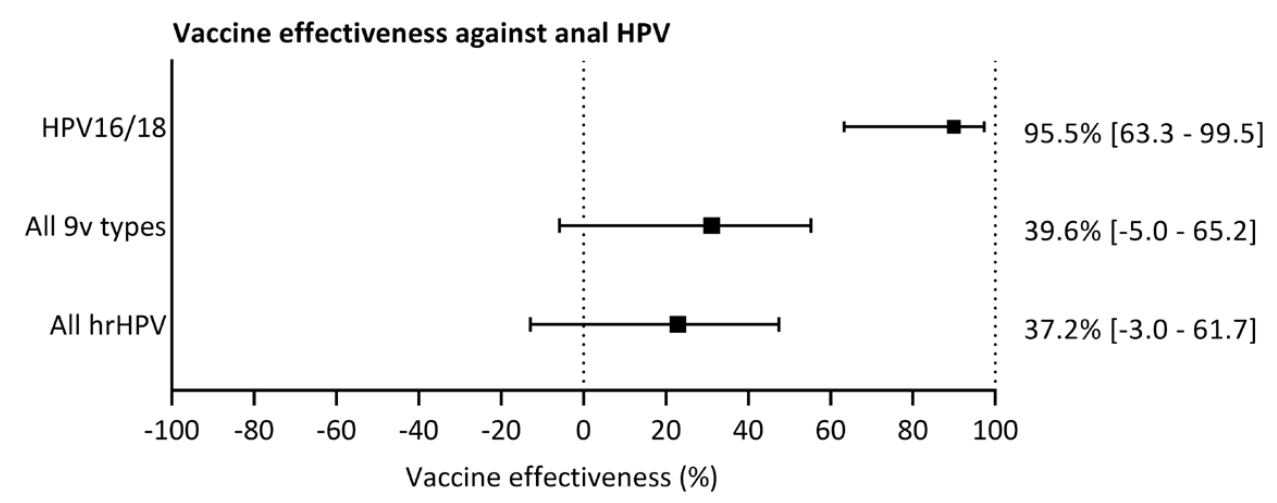

Supplementary Figure 4.3. Adjusted VE, restricted to women who were offered vaccination $\geq 5$ years ago (range $5-8$ years).

Note: All 9v types included all types of the 9vHPV vaccine: HPV6/11/16/18/31/33/45/52/58. All hrHPV types included: HPV16/18/31/33/35/39/45/51/52/56/58/59. VE was estimated as (1- odds ratio)*100\% and was adjusted for age, education level, anal sex in the past 6 months, number of sex partners in the past 6 months, STI-related symptoms, history of hormonal contraceptives use, and age vaccination was offered. 



\section{Part 2}

Population-level impact on HPV positivity 



\section{Chapter 5}

\section{Assessment of herd effects among women and heterosexual men after girls-only HPV16/18 vaccination in the Netherlands: A repeated cross- sectional study}

Petra J. Woestenberg Johannes A. Bogaards

Audrey J. King

Suzan Leussink

Marianne A. B. van der Sande

Christian J. P. A. Hoebe

Birgit H. B. van Benthem

The contents of this chapter have been published in International Journal of Cancer

Int J Cancer. 2019; 144:2718-27 


\section{Abstract}

Data on the impact of human papillomavirus (HPV) vaccination on the population HPV prevalence are largely obtained from women. We assessed the impact of the girls-only HPV16/18 vaccination program in the Netherlands that started in 2009, on trends in HPV prevalence among women and heterosexual men, using data from the PASSYON study. In this cross-sectional study, the HPV prevalence among 16- to 24-year-old visitors to sexually transmitted infection clinics was assessed in 2009, 2011, 2013, and 2015. We compared the genital post-vaccination HPV prevalence with the pre-vaccination prevalence (2009) using Poisson GEE models. In total, we included 4,996 women and 1,901 heterosexual men. The percentage of women who reported to be vaccinated increased from $2.3 \%$ in 2009 to $37 \%$ in 2015. Among all women, the HPV16/18 prevalence decreased from $23 \%$ pre-vaccination to $15 \%$ in 2015 (adjusted prevalence ratio [aPR] 0.62, p ptrend<.01). Among heterosexual men, the HPV16/18 prevalence decreased from 17\% pre-vaccination to $11 \%$ in 2015 (aPR 0.52, ptrend<.01). Of the heterosexual men with a steady partner, HPV16/18 prevalence was lower among those whose steady partner had been vaccine-eligible in the national immunization program (aPR 0.13). Among unvaccinated women, the HPV16/18 prevalence in 2015 was not different from pre-vaccination. The decreasing HPV16/18 prevalence among heterosexual men and the reduced HPV16/18 prevalence among heterosexual men with a vaccine-eligible steady partner strongly suggests herd protection from girls-only vaccination. Absence of notable herd effects among unvaccinated women 6 years postvaccination may be due to the moderate vaccine uptake among girls in the Netherlands. 


\section{Introduction}

Human papillomavirus (HPV) is a sexually transmitted virus that plays a causal role in the development of anogenital and oropharyngeal cancers in both men and women [1]. To prevent HPV-related cancers, many countries have included HPV vaccination in their national immunization program (NIP), using one of the available vaccines that provide direct protection against 2, 4, or 9 HPV types, all including HPV16 and HPV18 [2].

In the Netherlands, the bivalent HPV (2vHPV) vaccine (Cervarix ${ }^{\circledR}$, GSK) is used in the NIP; to date, this has been a girls-only program [3]. In 2009, there was a catch-up campaign for girls born in 1993-1996 with 52\% that completed the 3-dose schedule [4]. Routine HPV vaccination was introduced in 2010 for girls in the year they turn 13 years old, with an initial 3-dose uptake of 56\% (birth cohort 1997). The uptake increased to $61 \%$ for birth cohort 2001, but decreased again to 53\% for birth cohort 2002. In 2014, routine HPV vaccination changed to a 2-dose schedule [5].

We previously reported on direct 2vHPV vaccine effectiveness using cross-sectional data from female sexually transmitted infection (STI) clinic visitors. We showed high effectiveness against the vaccine types HPV16 and HPV18 and cross-protection against other oncogenic HPV types [6]. These findings were reiterated in a longitudinal cohort study among vaccine-eligible girls [7].

The population-level impact of HPV vaccination programs also includes possible indirect effects, such as herd protection. So far, the population-level impact of HPV vaccination programs on the HPV prevalence has mainly been studied among women. Surveillance studies have shown a decrease in the HPV16/18 prevalence since the introduction of vaccination [8]. Some studies have also shown decreases in the HPV16/18 prevalence among unvaccinated women [9-11]. This decrease among unvaccinated women is attributed to herd protection in men, yet there is limited information about trends in HPV prevalence among men, especially after $2 \mathrm{vHPV}$ vaccination. One study has shown that the HPV16/18 prevalence in urine samples from men decreased from $5.0 \%$ pre-vaccination to $1.1 \%$ 2-4 years post- girls-only 2vHPV vaccination [12]. However, since the method of sample collection had changed, the authors were cautious in drawing conclusions. Because HPV16/18 are associated with the majority of HPV-related cancers in men, demonstrating herd protection for these types in heterosexual men is important for assessing the overall health gain from a girls-only HPV vaccination program $[13,14]$.

We assessed the population-level impact of the girls-only 2vHPV vaccination program in the Netherlands by studying trends in the prevalence of HPV vaccine and cross protective types from pre-vaccination up to 6 years post-vaccination. We included women as well as heterosexual men, and focused on unvaccinated women and heterosexual men with vaccine-eligible partners to study herd protection. We used data from the PASSYON 
(PApillomavirus Surveillance among STI clinic Youngsters in the Netherlands) study, a biennial cross-sectional study among visitors to STI clinics that had been designed to monitor the HPV vaccination program in the Netherlands.

\section{Methods}

\section{Study design and population}

The PASSYON study started in 2009 when HPV vaccination was implemented in the Netherlands. Young (16- to 24-year-old) people who visited STI clinics throughout the Netherlands were asked to participate in the study. In addition to the routine STI consultation, participants were asked to fill out a questionnaire regarding demographics, sexual behavior, and vaccination status. Moreover, they were asked to provide a selfcollected genital swab for HPV testing. Women were instructed to insert a swab (Copan Diagnostics, Italy) about $4 \mathrm{~cm}$ into the vagina until resistance was felt and to turn it around along the walls of the vagina. Men were instructed to firmly move the swab up and down the entire shaft, the glans, the coronal sulcus, and under the foreskin of the penis. More details about the PASSYON study have been published previously [15]. To explore trends in the HPV prevalence after implementation of HPV vaccination, the PASSYON study was repeated in 2011, 2013, and 2015 using the same study protocol. Participants could be included in multiple study rounds, but the probability of repeated consultations is low as we sampled for only 2 months in the same period every other year (Figure 5.1). The Dutch Medical Research Involving Human Subjects Act (Dutch acronym: WMO) does not apply for our study, because only for-the-researchers-anonymized-data were used and there were no (medical) interventions other than routine care. The Medical Ethical Committee of the University of Utrecht, the Netherlands, provided a waiver for full medical ethical review (protocol number 08/397). Data were obtained using a unique code per person and all participants gave informed consent.

\section{Laboratory methods}

HPV testing protocols were constant across all years and described in detail elsewhere [15]. Briefly, DNA was extracted using the MagnaPure platform (Total Nucleic Acid Isolation Kit, Roche, the Netherlands) and HPV DNA was amplified using the SPF10 primer set and detected using the DNA enzyme-linked immunoassay (HPV-DEIA, DDL Diagnostics Laboratory, the Netherlands). Positive samples were genotyped with line-probe assay (HPVLiPA25, DDL Diagnostics Laboratory, the Netherlands), which is able to detect 25 HPV types, including HPV16 and HPV18. 


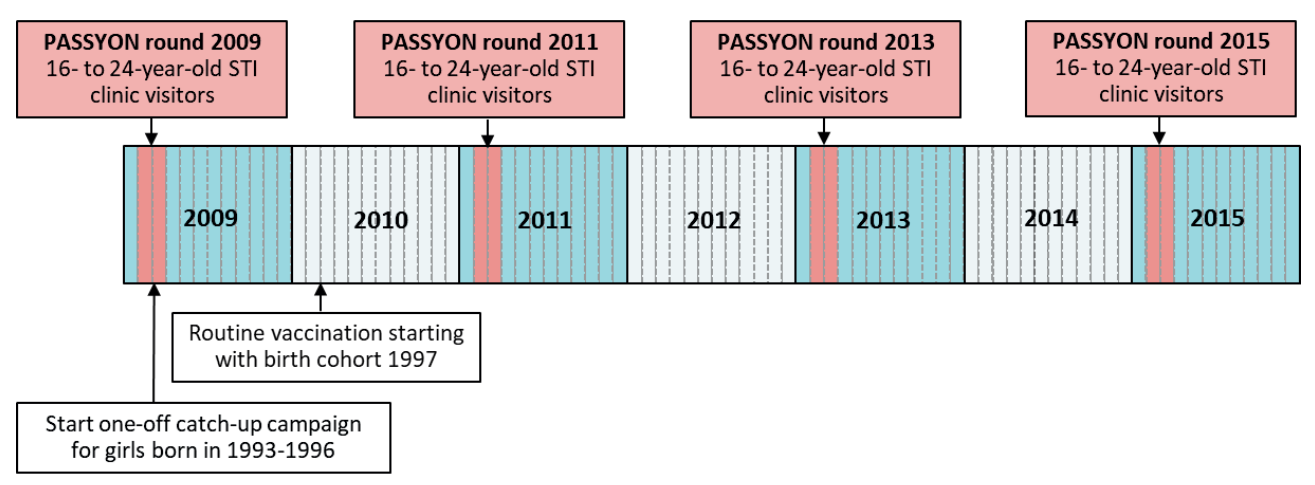

Figure 5.1. HPV vaccination in the Netherlands and the PASSYON study design.

\section{Statistical analyses}

Only participants with a genital swab were included in the analyses. All analyses were performed separately for all women (irrespective of vaccination status), heterosexual men (based on self-identified sexual preference), and unvaccinated women (based on selfreported vaccination status).

We calculated the prevalence and Wilson score $95 \%$ confidence interval $(95 \% \mathrm{Cl})$ of HPV16 and HPV18 (combined and separately) for each PASSYON year and performed a crude Cochran-Armitage Trend Test. Next, we compared the HPV prevalence of the postvaccination periods (2011, 2013, and 2015) with the pre-vaccination period (2009) and calculated prevalence ratios (PRs) using a Poisson model with robust error variance. This results in comparable estimates as compared to log-binomial regression, and improves numerical convergence [16]. Additionally, because we assumed identical effects of covariates on the prevalence of HPV types included in the analyses, we made use of a generalized estimating equation (GEE) model with an exchangeable correlation structure. This allows efficient estimation of coefficients and calculation of the population-averaged effect of study year on the HPV prevalence, either type-specific or pooled (as a weighted average) [17]. Linear trends over time were assessed by including PASSYON year as a continuous variable in the model. These analyses were adjusted for age (16-20 and 21-24 years) and possible confounders, presented in Table 5.1. The variables age at sexual debut and number of sex partners in the past 6 months and lifetime were categorized for analyses purposes based on knowledge about the HPV risk and size of each category. The selection of confounders was based on the following procedure. First, we explored the association with PASSYON year and high-risk HPV (hrHPV) positivity (being positive for HPV16/18/31/ $33 / 35 / 39 / 45 / 51 / 52 / 56 / 58 / 59)$, using $\chi^{2}$ tests. Using hrHPV instead of HPV16/18 positivity for the selection of confounders gave more power to detect possible associations. Variables associated with PASSYON year and hrHPV positivity $(p<.05)$ in univariable analyses were 
selected. Second, because sexual risk behavior variables were highly correlated, we used computerized selection models (stepwise with $\mathrm{p}<.05$ as entry and stay criteria) with hrHPV positivity as an outcome and the sexual risk behavior variables that were selected as independent variables. Variables that were included in the final selection model were evaluated as possible confounders to adjust for in the Poisson GEE models for comparing HPV prevalence between study rounds. For all women, we also adjusted for self-reported vaccination status to assess if possible trends in HPV prevalence over time was explained by an increasing proportion of vaccinated women in our study population. Although HPV vaccination was not offered to men in the Dutch NIP, it is possible that men were vaccinated elsewhere. In sensitivity analyses, we excluded heterosexual men who reported to have been HPV vaccinated. To study the population-level impact and herd protection for the cross-protective types HPV31/33/45 [9], we repeated the analyses by also including these types.

Because women who were offered vaccination in the Netherlands (women born in 1993 or later), were aging over the span of the PASSYON study, the vaccination coverage by age category differed over the years. We assessed for effect modification by age category by including an interaction term between PASSYON year and age category. For all women, we again additionally adjusted for self-reported vaccination status to assess if the possible difference in trends by age category were explained by differences in vaccination coverage. We also calculated the adjusted PRs (aPRs) and the trend for the age categories separately.

If participants reported being in a relationship, the age of the steady partner was asked. For heterosexual men, we assessed if the steady partner had been eligible for HPV vaccination in the Dutch NIP based on the reported age of the steady partner. We assumed the steady partner had been eligible for vaccination if she was $\leq 17$ years in PASSYON round 2011, $\leq 19$ years in PASSYON round 2013, and $\leq 21$ years in PASSYON round 2015. If the steady partner was older in a specific PASSYON round, we assumed she had not been eligible for HPV vaccination in the NIP. Also for all heterosexual men included in PASSYON round 2009, we assumed the steady partner had not been eligible for HPV vaccination. To consider herd effects, we calculated the combined HPV16/18 prevalence among heterosexual men by age of the steady partner and vaccine-eligibility of the steady partner. Next, we assessed the difference in HPV16/18 prevalence between heterosexual men with or without a vaccine-eligible steady partner by using a GEE model with HPV16/18 as an outcome and vaccine-eligibility of the steady partner as an independent variable. This analysis was adjusted for age of the men and age of the steady partner.

All analyses were performed using SAS version 9.4 (SAS Institute Inc., Cary, NC). We used a significance level of $p<.05$. We did complete case analyses, as none of the variables had more than $5 \%$ missing. 


\section{Results}

\section{Study population}

A total of 7,108 women and heterosexual men participated in the PASSYON study, of whom 6,897 (4,996 women and 1,901 heterosexual men) delivered a genital swab and were included in the current analysis; 1,524 in 2009, 1,775 in 2011, 1,816 in 2013, and 1,782 in 2015. The proportion of women who had been eligible for vaccination increased from $0.4 \%$ in 2009 to $5.3 \%$ in $2011,27 \%$ in 2013 , and $57 \%$ in 2015 . Of the women who had been eligible for vaccination, $55 \%(n=650)$ reported to be vaccinated (30 reported to be vaccinated with 1 dose, 42 with 2 doses, 456 with 3 doses, and 122 an unknow number of doses). The proportion of all women reporting to be vaccinated at least once increased from $2.3 \%$ in 2009 to $37 \%$ in 2015 . In total 27 heterosexual men (1.4\%) reported to be HPV vaccinated. Characteristics of the study population of all PASSYON years combined are presented in Table 5.1.

Table 5.1. Characteristics of the study population of all PASSYON years combined for all women, heterosexual men, and unvaccinated women.

\begin{tabular}{|c|c|c|c|}
\hline & $\begin{array}{l}\text { All women } \\
\text { N (\%) }\end{array}$ & $\begin{array}{l}\text { Heterosexual men } \\
\mathrm{N}(\%)\end{array}$ & $\begin{array}{l}\text { Unvaccinated women } \\
\mathbf{N}(\%)\end{array}$ \\
\hline Total & 4996 & 1901 & 3594 \\
\hline \multicolumn{4}{|l|}{ Age } \\
\hline $16-20$ years & $2012(40.3)$ & $557(29.3)$ & $1186(33.0)$ \\
\hline $21-24$ years & 2984 (59.7) & $1344(70.7)$ & $2408(67.0)$ \\
\hline \multicolumn{4}{|c|}{ Self-defined ethnicity } \\
\hline Dutch & 4319 (86.5) & $1522(80.1)$ & 3127 (87.1) \\
\hline Not Dutch & $675(13.5)$ & 377 (19.9) & 465 (12.9) \\
\hline \multicolumn{4}{|l|}{ Education levela $^{\mathrm{a}}$} \\
\hline Low/middle & $1246(25.1)$ & $591(31.2)$ & $835(23.3)$ \\
\hline High & 3719 (74.9) & $1303(68.8)$ & $2745(76.7)$ \\
\hline \multicolumn{4}{|l|}{ Sexual preference } \\
\hline Heterosexual & 4804 (96.2) & 1901 (100) & 3457 (96.2) \\
\hline Gay or bisexual & $192(3.8)$ & - & $137(3.8)$ \\
\hline \multicolumn{4}{|c|}{ Age at sexual debut ${ }^{b}$} \\
\hline$\leq 14$ years & $647(13.1)$ & $322(17.1)$ & $439(12.3)$ \\
\hline $15-16$ years & $2396(48.5)$ & $762(40.5)$ & 1697 (47.7) \\
\hline$\geq 17$ years & $1898(38.4)$ & $799(42.4)$ & $1423(40.0)$ \\
\hline \multicolumn{4}{|c|}{ No. of sex partners, past 6 months $^{c}$} \\
\hline 0-1 partners & $1627(32.6)$ & $418(22.0)$ & 1203 (33.5) \\
\hline 2-3 partners & $2412(48.3)$ & $715(37.6)$ & 1721 (47.9) \\
\hline 4-5 partners & $687(13.8)$ & $390(20.5)$ & $499(13.9)$ \\
\hline$\geq 6$ partners & $265(5.3)$ & 378 (19.9) & 169 (4.7) \\
\hline
\end{tabular}


Table 5.1. (Continued).

\begin{tabular}{|c|c|c|c|}
\hline & $\begin{array}{l}\text { All women } \\
\mathrm{N}(\%)\end{array}$ & $\begin{array}{l}\text { Heterosexual men } \\
\mathrm{N}(\%)\end{array}$ & $\begin{array}{l}\text { Unvaccinated women } \\
\mathrm{N} \text { (\%) }\end{array}$ \\
\hline \multicolumn{4}{|c|}{ No. of sex partners, lifetime $e^{c}$} \\
\hline$\leq 2$ partners & $570(11.6)$ & $105(5.8)$ & $396(11.2)$ \\
\hline 3-4 partners & $973(19.8)$ & $202(11.1)$ & $688(19.4)$ \\
\hline 5-6 partners & $966(19.7)$ & $240(13.2)$ & $694(19.6)$ \\
\hline 7-14 partners & $1702(34.7)$ & $585(32.2)$ & $1245(35.2)$ \\
\hline$\geq 15$ partners & $693(14.1)$ & $687(37.8)$ & $516(14.6)$ \\
\hline \multicolumn{4}{|c|}{ Anal sex past 6 months } \\
\hline No & $4351(87.6)$ & $1590(84.8)$ & $3122(87.3)$ \\
\hline Yes & $614(12.4)$ & $284(15.2)$ & $455(12.7)$ \\
\hline \multicolumn{4}{|l|}{ Notified for STIs ${ }^{d}$} \\
\hline No & $4511(90.6)$ & $1608(85.0)$ & $3263(91.1)$ \\
\hline Yes & $467(9.4)$ & $284(15.0)$ & 319 (8.9) \\
\hline \multicolumn{4}{|c|}{ STI-related symptoms ${ }^{d}$} \\
\hline No & 3799 (76.5) & $1367(72.4)$ & $2721(76.1)$ \\
\hline Yes & $1170(23.5)$ & $521(27.6)$ & $853(23.9)$ \\
\hline \multicolumn{4}{|l|}{ History of any STI } \\
\hline No & $2852(57.4)$ & $1055(55.7)$ & $2101(58.7)$ \\
\hline Yes & $1266(25.5)$ & 377 (19.9) & $920(25.7)$ \\
\hline Never tested & $851(17.1)$ & $462(24.4)$ & $558(15.6)$ \\
\hline \multicolumn{4}{|c|}{ Current genital chlamydia infection ${ }^{d}$} \\
\hline No & $4283(86.1)$ & $1594(84.4)$ & $3098(86.5)$ \\
\hline Yes & $694(13.9)$ & $294(15.6)$ & $482(13.5)$ \\
\hline \multicolumn{4}{|l|}{ Steady partner } \\
\hline No & $2961(60.7)$ & 1037 (56.5) & $2127(60.6)$ \\
\hline Yes, for 0-6 months & $1102(22.6)$ & 475 (25.9) & $801(22.8)$ \\
\hline Yes, for $\geq 6$ months & $813(16.7)$ & $324(17.6)$ & $583(16.6)$ \\
\hline \multicolumn{4}{|c|}{ Condom use with casual partners, past 6 months ${ }^{e}$} \\
\hline Inconsistent & $1950(39.2)$ & $851(44.9)$ & $1344(37.5)$ \\
\hline Consistent & $1806(36.3)$ & $658(34.7)$ & $1361(37.9)$ \\
\hline No casual partners & $1224(24.6)$ & $385(20.3)$ & $882(24.6)$ \\
\hline
\end{tabular}

Totals vary because of missing values.

a. High educational level included school of higher general secondary education, pre-university education, university of applied sciences, and university. Low/middle educational level included all other levels of education.

b. Categorized for analyses purposes. Minimum-maximum age reported: 9-24 years among (unvaccinated) women and heterosexual men.

c. Categorized for analyses purposes. Maximum no. of partners reported: 540 past 6 months and 900 lifetime among (unvaccinated) women; 50 past 6 months and 400 lifetime among heterosexual men. $d$ Based on information of the STI clinic visit.

e. Inconsistent included reporting never, rarely and "sometimes I do, sometimes I do not" condom use. Consistent included reporting often or always condom use. 
In general, the indicators for sexual risk behavior increased over the years among women, heterosexual men, and unvaccinated women (Supplementary Tables 5.1, 5.2, 5.3). For example, we observed an association between lifetime number of sex partners and PASSYON year; with proportions reporting $\geq 15$ lifetime sex partners of $12 \%$ in 2009 and $18 \%$ in 2015 among all women; 31\% in 2009 and 44\% in 2015 among heterosexual men; and 12\% in 2009 and 19\% in 2015 among unvaccinated women. The genital chlamydia prevalence was associated with PASSYON year among heterosexual men only. Supplementary Tables 5.1, 5.2, 5.3 also show the association between the characteristics and hrHPV positivity. In general, people with higher sexual risk behavior were more often hrHPV positive.

\section{HPV prevalence over time}

Figure 5.2 presents the HPV16 and HPV18 prevalence over time and the crude trend test among all women, heterosexual men, and unvaccinated women. Among all women, the HPV16/18 prevalence decreased from 23\% in 2009 to 15\% in 2015 (aPR 0.62, Table 5.2). Also for HPV16 and HPV18 separately, there was a significant decrease over time (aPR 0.59 and 0.69 respectively). When we additionally adjusted for vaccination status, the prevalences in 2015 were no longer significantly different from 2009. Among heterosexual men, the combined HPV16/18 prevalence decreased from 17\% in 2009 to $11 \%$ in 2015 (aPR 0.52). Also separately, HPV16 and HPV18 prevalences were significantly lower in 2015 compared to 2009 (aPR 0.64 and 0.33 respectively). Excluding the 27 heterosexual men who reported to be vaccinated did not lead to different results (Supplementary Table 5.4). Among unvaccinated women, we observed no declining trends in the HPV16 or HPV18 prevalence.

For HPV31, HPV33, and HPV45, we only observed a declining trend in the HPV31 prevalence among all women (adjusted ptrend 0.01 ), but not among heterosexual men or unvaccinated women. For the other HPV types, no trends were observed except for an increasing trend of HPV45 among unvaccinated women (Supplementary Table 5.5).

The vaccination coverage over time differed by age category; for example in $2013,40 \%$ of the 16- to 20 -year-old women reported to be vaccinated ( $\geq 1$ dose), while $4.6 \%$ of the 21 to 24-year-old women reported to be vaccinated. We observed that the HPV16/18 prevalence among 16- to 20-year-old women decreased faster as compared to 21- to 24year-old women (aPR 0.41 vs 0.74 respectively for 2015 compared to 2009, Supplementary Figure 5.1 and Supplementary Table 5.6). The difference in the effect of year by age group was statistically significant $(p<.01)$. After additional adjustment for vaccination status, the difference between ages was no longer statistically significant. Among heterosexual men and unvaccinated women, there was no statistically significant interaction with age. Among unvaccinated women, there were no statistically significant trends in the HPV16 or HPV18 prevalence for both age categories (Supplementary Table 5.6). 
A) All women

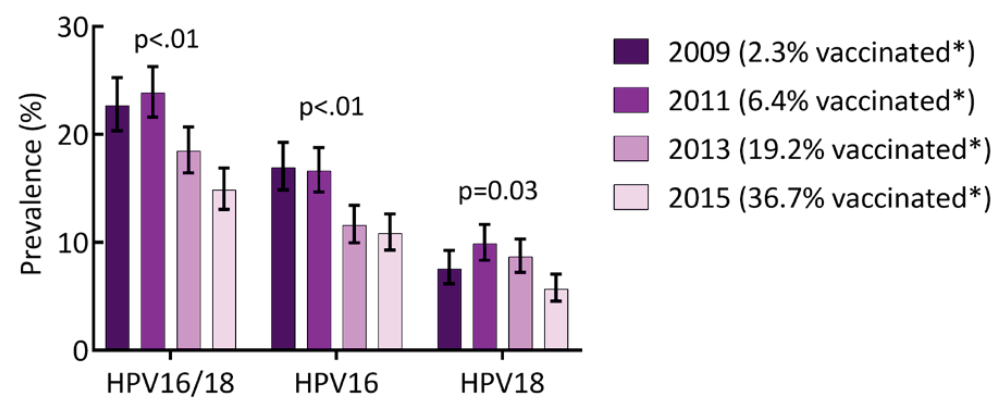

B) Heterosexual men

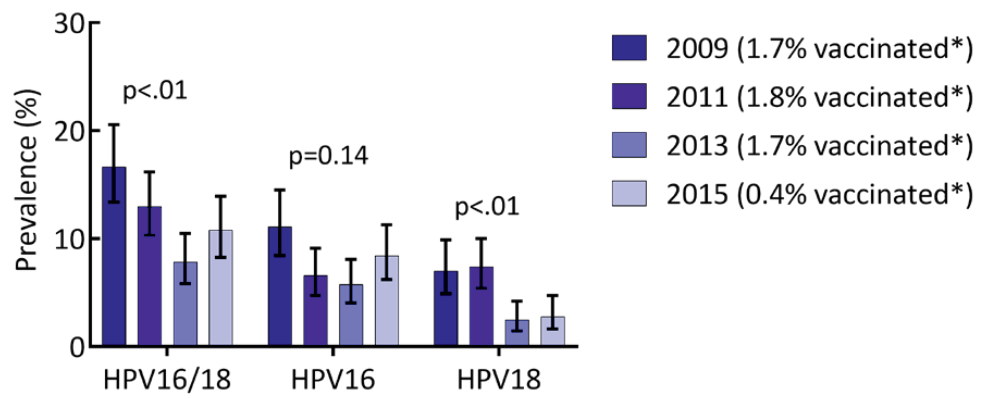

C) Unvaccinated women

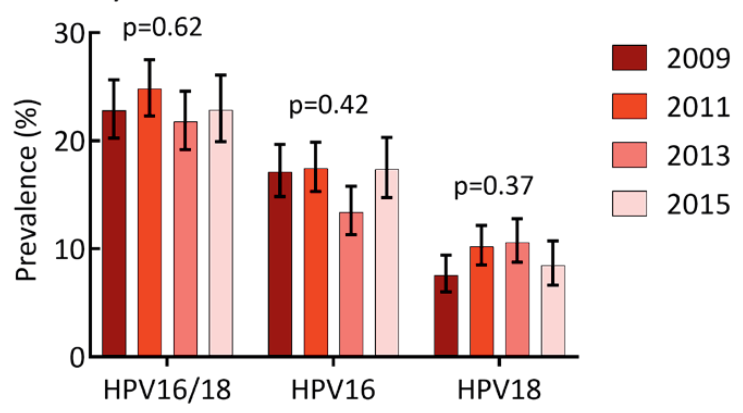

Figure 5.2. Prevalence of HPV types 16 or 18, HPV16, and HPV18 over time and $p$ values for the crude trend test, among A) all women; B) heterosexual men; C) unvaccinated women.

Note: The $p$ value presents the Cochran-Armitage Trend Test. *Percentage of women and heterosexual men who reported to be vaccinated at least once. 


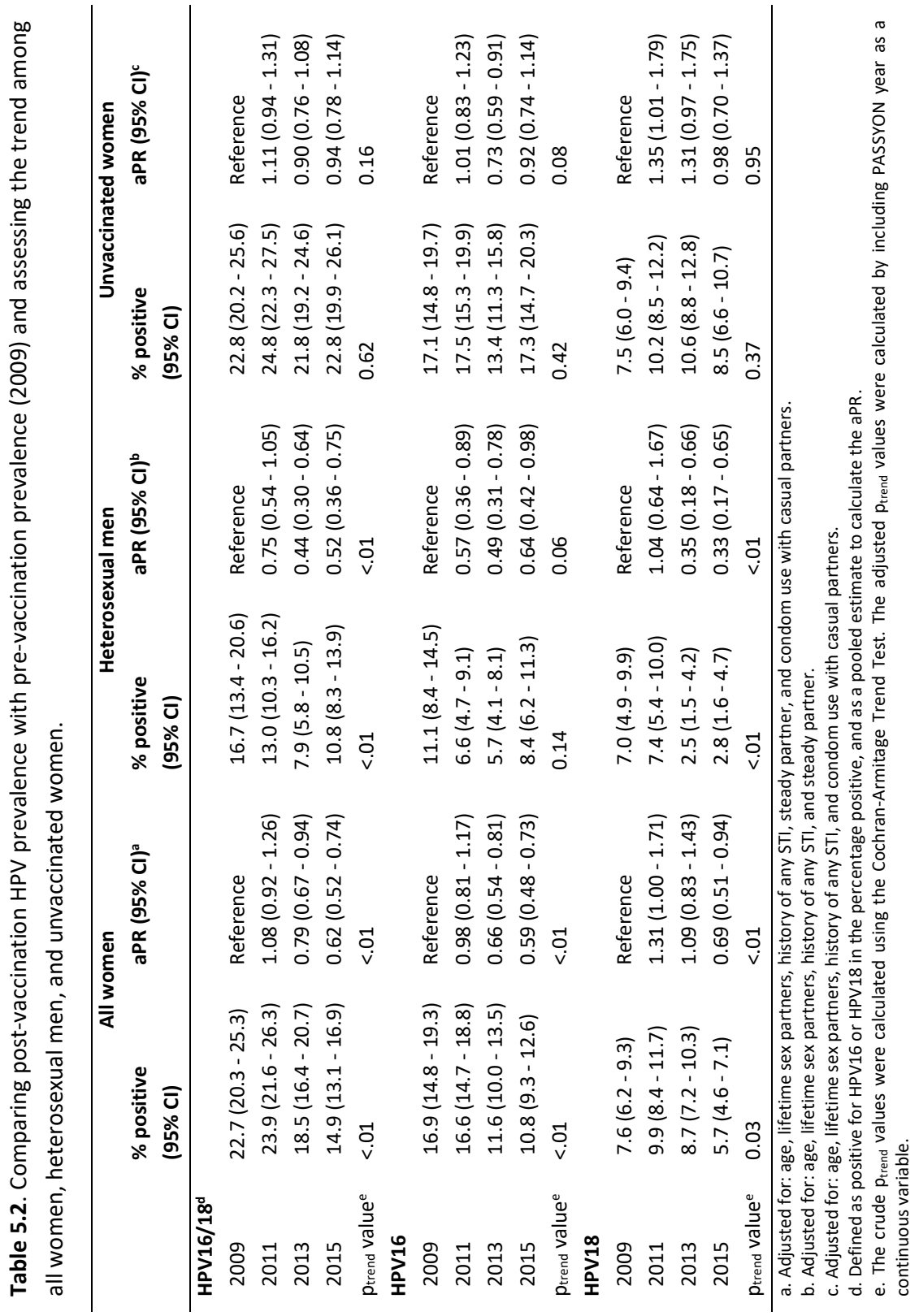




\section{Vaccine-eligible steady partner}

The proportion of heterosexual men reporting a steady partner who had been eligible for HPV vaccination increased from $2.2 \%$ in 2011 , to $15 \%$ in 2013 , and $19 \%$ in 2015 . Figure 5.3 shows the combined HPV16/18 prevalence among heterosexual men according to the age of the steady partner and vaccine-eligibility of the steady partner. Overall, heterosexual men whose steady partner had been vaccine-eligible were less often HPV16/18 positive compared to heterosexual men whose steady partner had not been vaccine-eligible in the NIP (aPR 0.13 [95\% Cl 0.04-0.41]).

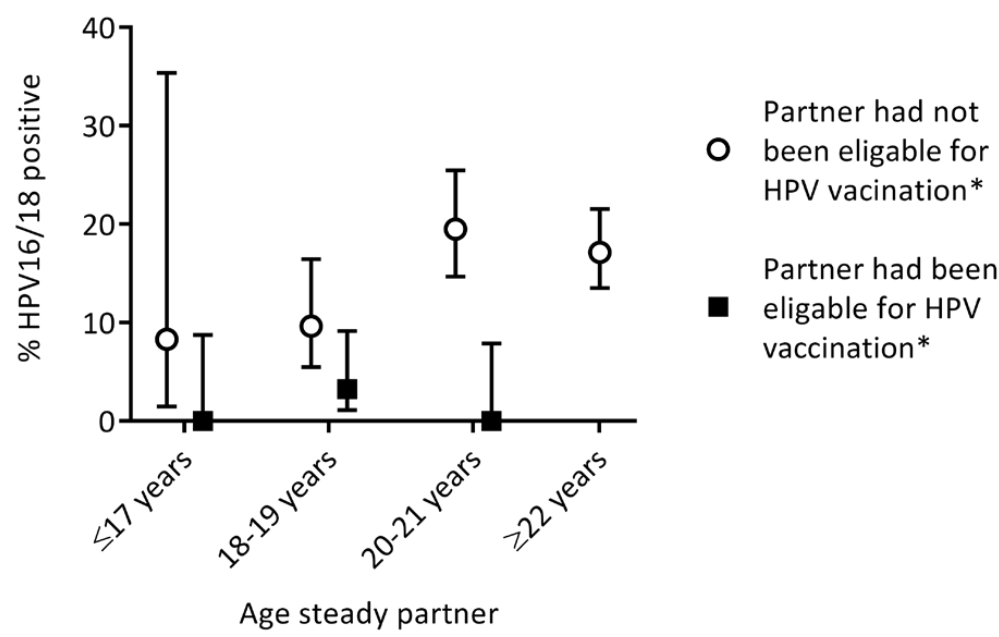

Figure 5.3. Prevalence of HPV types 16 or 18 among heterosexual men who reported to have a steady partner, by age of the steady partner and vaccine-eligibility of the steady partner.

Note: *Vaccine-eligibility of the steady partner was based on the reported age of the steady partner in a specific PASSYON study round and the Dutch NIP. None of the steady partners of $\geq 22$ years had been eligible according to the NIP in the Netherlands.

\section{Discussion}

We estimated the population-level impact of the national girls-only 2vHPV vaccination program in the Netherlands by comparing HPV prevalence from pre-vaccination to postvaccination periods among male and female visitors to STI clinics. We showed decreasing trends in the HPV16 and HPV18 prevalence among all women and heterosexual men, but not among unvaccinated women separately. Of the heterosexual men who reported to have a steady partner, HPV16/18 prevalence was lower among those whose steady partner had been vaccine-eligible.

Our results provide compelling evidence for herd protection for the vaccine types among men in the aftermath of girls-only HPV16/18 vaccination and show that herd effects among 
heterosexual men will likely precede those among unvaccinated women. Our data offer empirical support for the population-level impact of vaccination against the 2 most oncogenic HPV types as previously predicted by transmission-dynamic models [18].

We do acknowledge some limitations. First, STI clinics became stricter in prioritizing high-risk individuals especially since 2015, when the funding of the STI clinics had changed [19]. We indeed observed increased sexual risk behavior over time possibly related to changes in the access policy of STI clinics. Although we adjusted for known changes, unknown changes in the study population may have resulted in changes in the HPV prevalence unrelated to HPV vaccination. This could for instance explain the observed increase in HPV16 prevalence in 2015 compared to 2013 among heterosexual men. If participants in the post-vaccination study periods were at higher HPV risk, we may have underestimated the impact of vaccination, including declines in HPV16/18 prevalence among unvaccinated women. However, the chlamydia prevalence did not increase among (unvaccinated) women, suggesting that unrecorded sexual risk behavior likely did not chance that much among female study participants. Analyses restricted to chlamydia positive unvaccinated women did not lead to different results (results not shown). Second, the use of self-reported vaccination status may have induced bias. Among women, we believe the bias will be minimal as we previously showed that the HPV16 and HPV18 antibody concentrations agreed well with the self-reported vaccination status [6]. Of the heterosexual men, $1.4 \%$ reported to be HPV vaccinated. If these men were truly vaccinated against HPV, this would lead to an overestimation of the herd effects. However, excluding the men who reported to be HPV vaccinated did not lead to different results. We also used self-reported sexual preference to identify heterosexual men. It might be that some men did (also) have sex with men. Such bias would have underestimated the impact of vaccination. Third, only 1 pre-vaccination measurement was available. If there were natural fluctuations in the HPV prevalence in the absence of vaccination, multiple pre-vaccination measurements would have been preferred to obtain an accurate estimate of the average pre-vaccination prevalence and to assess possible pre-vaccination trends. Last, we used a population of visitors to STI clinics who are at higher HPV risk as compared to the general population. The results are therefore not representative of the general Dutch population, probably underestimating the impact of vaccination [20].

The decrease in the HPV16/18 prevalence among women in our study, coincided with an increase in the percentage of women who reported to be vaccinated. After adjustment for vaccination status, the HPV16/18 prevalence did not differ in 2015 as compared to 2009, indicating that the increasing proportion of vaccinated women explained the decreasing HPV prevalence. In other countries, also a decline in the HPV16/18 prevalence among women was observed after introduction of 2vHPV vaccination. In England, the HPV16/18 
prevalence decreased from $18 \%$ pre-vaccination to $4.0 \% 4-5$ years post-vaccination among 16- to 18-year-old sexually active women [21]. In Scotland, the HPV16/18 prevalence decreased from $30 \%$ pre-vaccination to $4.5 \% 7$ years post-vaccination among 20 - to 21 -yearold women who underwent their first cervical screening. The larger declines in these countries as compared to our study could be explained by an overall lower percentage of women vaccinated in our study $(37 \% \geq 1$ dose in 2015$)$. This reflects both a lower percentage of women who had been eligible for vaccination (57\% in 2015) and a lower vaccination uptake among vaccine-eligible women ( $55 \% \geq 1$ dose). Among 16- to 20 -year-old women, with a higher percentage vaccinated, we observed larger declines in the HPV16/18 prevalence.

We also observed a decrease in the HPV16/18 prevalence among heterosexual men since the introduction of girls-only $2 \mathrm{vHPV}$ vaccination. Our results are comparable to Australia where a declining trend in the HPV16/18 prevalence among heterosexual men was observed after girls-only quadrivalent HPV vaccination [22]. In Australia, also a decline in the HPV16/18 prevalence was observed among foreign-born heterosexual men who had arrived from countries with a $2 \mathrm{vHPV}$ vaccination program within 2 years of study inclusion. However, effects of exposure within those countries might have been negligible because the majority of the HPV16/18 infections clear within 2 years [23].

While the decreasing HPV16/18 prevalence among heterosexual men in our study strongly suggests herd protection, causality cannot be concluded based on ecological analyses. Nonetheless, the decreasing prevalence in combination with a lower HPV16/18 prevalence among men whose steady partner had been vaccine-eligible strongly indicates that heterosexual men receive indirect protection. With most HPV-related penile cancers attributed to HPV16 [24], cancer reductions are also expected to occur for heterosexual men in the aftermath of girls-only HPV vaccination. Among men who have sex with men, large reductions in HPV-related cancers are not expected, because they benefit less from herd protection after girls-only vaccination [25]. While it is anticipated that HPV prevalence will also decline at other anatomical sites among heterosexual men, this has not yet been demonstrated. Given that oropharyngeal cancers constitute the largest HPV-related burden in men [26], showing herd effects against oral HPV is valuable to acknowledge the ultimate impact of HPV vaccination.

Among heterosexual men, the decline in HPV prevalence was larger for HPV18 than for HPV16, which is in line with data from Finland where also larger herd effects were observed for HPV18 [10]. Higher herd effects for HPV18 could be explained by a lower basic reproduction number (as a consequence of a higher clearance relative to HPV16) [23, 27, 28]. Even though HPV31/33/45 could also be expected to have a lower basic reproduction number than HPV16, there were no signs of herd effects for these types. This could be 
related to a relatively low background prevalence in combination with reduced vaccine effectiveness, resulting in limited power to detect herd effects against cross-protective types compared to the vaccine types.

Because the vaccination coverage of completed schedule among vaccine-eligible women in the Netherlands is 50\%-60\% [5], herd protection will not have reached its full potential $[13,14]$. This is particularly true for herd protection in unvaccinated heterosexual women, which is derived from herd protection in heterosexual men, and thus constitutes a second-order effect. With suboptimal girls-only vaccination coverage, vaccinating boys along with girls will not only protect boys themselves, but could also increase herd protection to unvaccinated women $[29,30]$. Based on modeling studies, $80 \%$ vaccination coverage in both men and women, but not in either sex, could eradicate the vaccine types [18].

We did not find signs of herd effects among unvaccinated women, also not when stratified by age. Nonetheless, other studies have observed a declining prevalence of the HPV vaccine types among unvaccinated women suggestive of herd protection. In Scotland, unvaccinated women born in 1995 were less often HPV16/18 positive at their first cervical screening compared to women born in 1988 who were not eligible for vaccination $(5.3 \%$ vs 30\%) [9]. Also in Australia and the United States, decreases in the vaccine-type prevalence have been recorded among unvaccinated women [31, 32]. There are several possible explanations for the absence of a declining trend among unvaccinated women in our study. First, in high-risk populations with frequent changes in sex partners, people are more likely to encounter a HPV-positive man or women, limiting herd effects [20]. However, in Australia declines in vaccine-type prevalence were also observed among the high-risk group of chlamydia-positive unvaccinated women [33]. Second, in Australia and Scotland, the vaccination initiation rate was much higher: $>80 \%$ in Australia and $>90 \%$ in Scotland [2]. Third, the time horizon of our study ( 6 years post-vaccination) might be too short to observe second-order herd effects. In the United States, where vaccination coverage was also limited, decreases in vaccine-type prevalence among unvaccinated women were noted 5-8 years after vaccine introduction and not yet after 3-6 years [32, 34].

In conclusion, the declining HPV16/18 prevalence among women is consistent with previous studies, but our findings also provide evidence for herd protection in heterosexual men after girls-only HPV16/18 vaccination. Due to the reduction in the HPV16/18 prevalence among women and heterosexual men, HPV-related cancers are expected to decline in both sexes after girls-only HPV vaccination. The absence of measurable herd effects among unvaccinated women 6 years post-vaccination highlights once again the importance of high vaccination coverage to optimally reduce HPV-related cancer morbidity. 


\section{References}

1. Chaturvedi AK. Beyond cervical cancer: burden of other HPV-related cancers among men and women. J Adolesc Health 2010; 46:S20-6.

2. Bruni L, et al. Global estimates of human papillomavirus vaccination coverage by region and income level: a pooled analysis. Lancet Glob Health 2016; 4:e453-63.

3. de Melker HE, et al. [Introductie van vaccinatie tegen baarmoederhalskanker]. NTvG 2009; 153:65861.

4. van Lier EA, et al. [Vaccinatiegraad Rijksvaccinatieprogramma Nederland: Verslagjaar 2011]. Bilthoven: RIVM, 2011. Nr: 210021014/2011.

5. van Lier EA, et al. [Vaccinatiegraad en jaarverslag Rijksvaccinatieprogramma Nederland 2016]. Bilthoven: RIVM, 2017. Nr: 2017-0010.

6. Woestenberg PJ, et al. Bivalent Vaccine Effectiveness Against Type-Specific HPV Positivity: Evidence for Cross-Protection Against Oncogenic Types Among Dutch STI Clinic Visitors. J Infect Dis 2018; 217:213-22.

7. Donken R, et al. High Effectiveness of the Bivalent Human Papillomavirus (HPV) Vaccine Against Incident and Persistent HPV Infections up to 6 Years After Vaccination in Young Dutch Women. J Infect Dis 2018; 217:1579-89.

8. Drolet $\mathrm{M}$, et al. Population-level impact and herd effects following human papillomavirus vaccination programmes: a systematic review and meta-analysis. Lancet Infect Dis 2015; 15:565-80.

9. Kavanagh $\mathrm{K}$, et al. Changes in the prevalence of human papillomavirus following a national bivalent human papillomavirus vaccination programme in Scotland: a 7-year cross-sectional study. Lancet Infect Dis 2017; 17:1293-302.

10. Lehtinen $M$, et al. Impact of gender-neutral or girls-only vaccination against human papillomavirusResults of a community-randomized clinical trial (I). Int J Cancer 2018; 142:949-58.

11. Mesher D, et al. The Impact of the National HPV Vaccination Program in England Using the Bivalent HPV Vaccine: Surveillance of Type-Specific HPV in Young Females, 2010-2016. J Infect Dis 2018; 218:911-21.
12. Sonnenberg $P$, et al. Prevalence, risk factors, and uptake of interventions for sexually transmitted infections in Britain: findings from the National Surveys of Sexual Attitudes and Lifestyles (Natsal). Lancet 2013; 382:1795-806.

13. Bogaards JA, et al. Direct benefit of vaccinating boys along with girls against oncogenic human papillomavirus: bayesian evidence synthesis. BMJ 2015; 350:h2016.

14. Qendri V, et al. Health and Economic Impact of a Tender-Based, Sex-Neutral Human Papillomavirus 16/18 Vaccination Program in the Netherlands. J Infect Dis 2017; 216:210-9.

15. Vriend $\mathrm{HJ}$, et al. Type-specific human papillomavirus infections among young heterosexual male and female STI clinic attendees. Sex Transm Dis 2012; 39:72-8.

16. Zou GY, et al. Extension of the modified Poisson regression model to prospective studies with correlated binary data. Stat Methods Med Res 2013; 22:661-70.

17. Xue $X$, et al. Marginal and mixed-effects models in the analysis of human papillomavirus natural history data. Cancer Epidemiol Biomarkers Prev 2010; 19:159-69.

18. Brisson $M$, et al. Population-level impact, herd immunity, and elimination after human papillomavirus vaccination: a systematic review and meta-analysis of predictions from transmissiondynamic models. Lancet Public Health 2016; 1:e8e17.

19. Visser M, et al. Sexually transmitted infections in the Netherlands in 2016. Bitlhoven: RIVM, 2017. Nr: 2017-0003; DOI: 10.21945/RIVM-2017-0003.

20. Baussano I, et al. Impacts of human papillomavirus vaccination for different populations: A modeling study. Int J Cancer 2018; 143:1086-92.

21. Mesher $D$, et al. Continuing reductions in HPV $16 / 18$ in a population with high coverage of bivalent HPV vaccination in England: an ongoing crosssectional study. BMJ Open 2016; 6:e009915.

22. Chow EPF, et al. Quadrivalent vaccine-targeted human papillomavirus genotypes in heterosexual men after the Australian female human papillomavirus vaccination programme: a 
retrospective observational study. Lancet Infect Dis 2017; 17:68-77.

23. Giuliano AR, et al. Incidence and clearance of genital human papillomavirus infection in men (HIM): a cohort study. Lancet 2011; 377:932-40.

24. Backes DM, et al. Systematic review of human papillomavirus prevalence in invasive penile cancer. Cancer Causes Control 2009; 20:449-57.

25. Chow EP, et al. Ongoing decline in genital warts among young heterosexuals 7 years after the Australian human papillomavirus (HPV) vaccination programme. Sex Transm Infect 2015; 91:214-9.

26. McDonald SA, et al. Disease burden of human papillomavirus infection in the Netherlands, 19892014: the gap between females and males is diminishing. Cancer Causes Control 2017; 28:203-14. 27. Brisson $M$, et al. Different population-level vaccination effectiveness for HPV types 16, 18, 6 and 11. Sex Transm Infect 2011; 87:41-3.

28. Ramanakumar AV, et al. Incidence and duration of type-specific human papillomavirus infection in high-risk HPV-naive women: results from the control arm of a phase II HPV-16/18 vaccine trial. BMJ Open 2016; 6:e011371.

29. Brisson $M$, et al. Incremental impact of adding boys to current human papillomavirus vaccination programs: role of herd immunity. J Infect Dis 2011; 204:372-6.

30. Lehtinen $M$, et al. Gender-neutral vaccination provides improved control of human papillomavirus types 18/31/33/35 through herd immunity: Results of a community randomized trial (III). Int J Cancer 2018; 143:2299-310.

31. Tabrizi SN, et al. Assessment of herd immunity and cross-protection after a human papillomavirus vaccination programme in Australia: a repeat crosssectional study. Lancet Infect Dis 2014; 14:958-66.

32. Oliver SE, et al. Prevalence of Human Papillomavirus Among Females After Vaccine Introduction-National Health and Nutrition Examination Survey, United States, 2003-2014. J Infect Dis 2017; 216:594-603.

33. Chow EP, et al. Human papillomavirus in young women with Chlamydia trachomatis infection 7 years after the Australian human papillomavirus vaccination programme: a cross-sectional study. Lancet Infect Dis 2015; 15:1314-23.

34. Markowitz LE, et al. Prevalence of HPV After Introduction of the Vaccination Program in the United States. Pediatrics 2016; 137:e20151968. 


\section{Supplementary information to Chapter 5}

Supplementary Table 5.1. Characteristics over time and relation with hrHPV positivity among all women.

\begin{tabular}{|c|c|c|c|c|c|c|c|}
\hline & \multirow{2}{*}{ 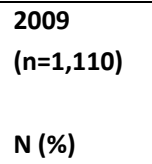 } & \multirow{2}{*}{$\begin{array}{l}2011 \\
(n=1,274) \\
N(\%)\end{array}$} & \multirow{2}{*}{$\begin{array}{l}2013 \\
(n=1,294) \\
N(\%)\end{array}$} & \multirow{2}{*}{$\begin{array}{l}2015 \\
(n=1,318) \\
N(\%)\end{array}$} & \multirow[b]{2}{*}{$\begin{array}{l}\text { p } \\
\text { value }^{\text {b }}\end{array}$} & \multicolumn{2}{|c|}{ hrHPV $^{a}$} \\
\hline & & & & & & $\begin{array}{l}\text { n positive } \\
\text { (\%) }\end{array}$ & $\begin{array}{l}p \\
\text { value }^{b}\end{array}$ \\
\hline Age & & & & & $<.01$ & & $<.01$ \\
\hline $16-20$ years & $480(43.2)$ & $465(36.5)$ & 539 (41.7) & $528(40.1)$ & & $1071(53.2)$ & \\
\hline $21-24$ years & $630(56.8)$ & $809(63.5)$ & $755(58.3)$ & 790 (59.9) & & $1921(64.4)$ & \\
\hline \multicolumn{2}{|c|}{ Self-defined ethnicity } & & & & 0.67 & & 0.30 \\
\hline Dutch & $964(87.0)$ & $1093(85.8)$ & $1113(86.0)$ & $1149(87.2)$ & & $2599(60.2)$ & \\
\hline Not Dutch & $144(13.0)$ & $181(14.2)$ & $181(14.0)$ & 169 (12.8) & & $392(58.1)$ & \\
\hline \multicolumn{2}{|l|}{ Education level $^{c}$} & & & & $<.01$ & & 0.79 \\
\hline Low/middle & $232(21.2)$ & $335(26.4)$ & $349(27.2)$ & $330(25.1)$ & & $750(60.2)$ & \\
\hline High & $863(78.8)$ & $933(73.6)$ & $936(72.8)$ & 987 (74.9) & & $2223(59.8)$ & \\
\hline \multicolumn{2}{|c|}{ Sexual preference } & & & & $<.01$ & & 0.76 \\
\hline Heterosexual & $1079(97.2)$ & 1235 (96.9) & 1241 (95.9) & $1249(94.8)$ & & 2875 (59.9) & \\
\hline Gay/bisexual & $31(2.8)$ & $39(3.1)$ & $53(4.1)$ & $69(5.2)$ & & $117(60.9)$ & \\
\hline \multicolumn{2}{|c|}{ Age at sexual debut } & & & & 0.07 & & 0.09 \\
\hline$\leq 14$ years & $118(10.7)$ & $158(12.6)$ & $193(15.0)$ & $178(13.7)$ & & $403(62.3)$ & \\
\hline $15-16$ years & $539(48.8)$ & $614(48.9)$ & $606(47.2)$ & $637(49.1)$ & & $1456(60.8)$ & \\
\hline$\geq 17$ years & $447(40.5)$ & $484(38.5)$ & 485 (37.8) & $482(37.2)$ & & $1103(58.1)$ & \\
\hline \multicolumn{3}{|c|}{ No. of sex partners, past 6 months } & & & $<.01$ & & $<.01$ \\
\hline 0-1 partner & $391(35.3)$ & $476(37.4)$ & $421(32.6)$ & $339(25.7)$ & & $820(50.4)$ & \\
\hline 2-3 partners & $528(47.7)$ & $599(47.1)$ & $649(50.2)$ & $636(48.3)$ & & $1486(61.6)$ & \\
\hline 4-5 partners & $141(12.7)$ & $152(12.0)$ & $156(12.1)$ & $238(18.1)$ & & $481(70.0)$ & \\
\hline$\geq 6$ partners & $48(4.3)$ & $45(3.5)$ & $67(5.2)$ & $105(8.0)$ & & $203(76.6)$ & \\
\hline \multicolumn{3}{|c|}{ No. of sex partners, lifetime } & & & $<.01$ & & $<.01$ \\
\hline$\leq 2$ partners & $138(12.6)$ & $183(14.6)$ & $128(10.1)$ & $121(9.4)$ & & $202(35.4)$ & \\
\hline 3-4 partners & $235(21.5)$ & $257(20.5)$ & 265 (20.9) & $216(16.7)$ & & $489(50.3)$ & \\
\hline 5-6 partners & $224(20.5)$ & $239(19.1)$ & $249(19.6)$ & $254(19.7)$ & & $543(56.2)$ & \\
\hline 7-14 partners & 360 (32.9) & $414(33.1)$ & $458(36.1)$ & $470(36.4)$ & & $1166(68.5)$ & \\
\hline$\geq 15$ partners & $136(12.4)$ & $159(12.7)$ & $169(13.3)$ & $229(17.8)$ & & $534(77.1)$ & \\
\hline \multicolumn{3}{|c|}{ Anal sex, past 6 months } & & & 0.09 & & 0.06 \\
\hline No & $964(87.5)$ & $1138(89.5)$ & $1119(87.1)$ & $1130(86.4)$ & & $2583(59.4)$ & \\
\hline Yes & $138(12.5)$ & $133(10.5)$ & 165 (12.9) & $178(13.6)$ & & $389(63.4)$ & \\
\hline \multicolumn{2}{|l|}{ Notified for STIs ${ }^{d}$} & & & & $<.01$ & & 0.02 \\
\hline No & $1027(92.8)$ & 1167 (91.9) & 1169 (90.5) & $1148(87.6)$ & & $2677(59.3)$ & \\
\hline Yes & $80(7.2)$ & $103(8.1)$ & $122(9.5)$ & $162(12.4)$ & & $303(64.9)$ & \\
\hline \multicolumn{3}{|c|}{ STI-related symptoms ${ }^{d}$} & & & 0.19 & & 0.73 \\
\hline No & 847 (76.9) & $993(78.3)$ & $962(74.7)$ & $997(76.0)$ & & 2268 (59.7) & \\
\hline Yes & $254(23.1)$ & $276(21.7)$ & $326(25.3)$ & $314(24.0)$ & & $705(60.3)$ & \\
\hline
\end{tabular}


Supplementary Table 5.1. (Continued).

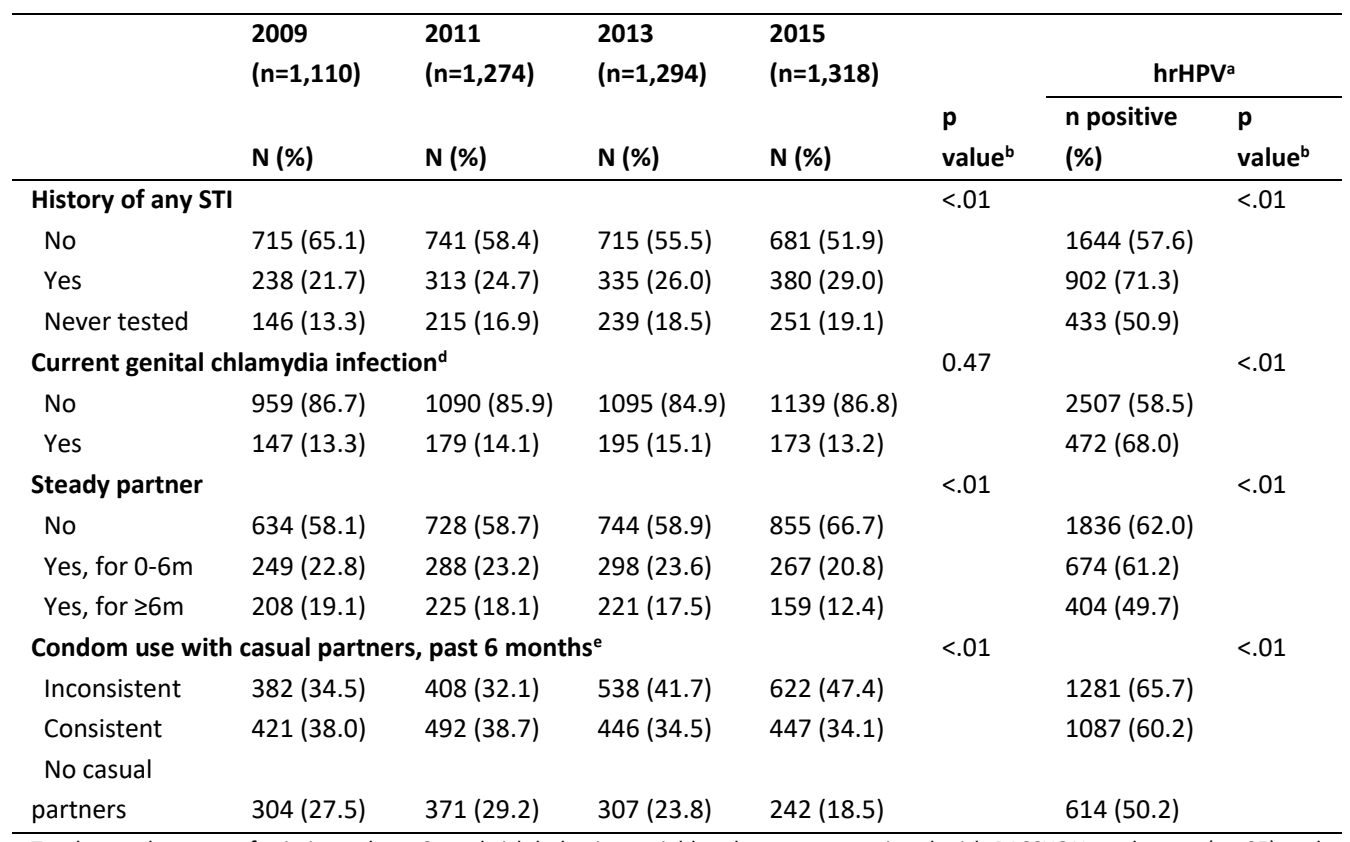

Totals vary because of missing values. Sexual risk behavior variables that were associated with PASSYON study year $(p<.05)$ and hrHPV $(p<.05)$ were considered in a computerized stepwise selection model, to select confounders to adjust for. The variables that were selected in the stepwise model were: lifetime sex partners, history of any STI, steady partner, and condom use with casual partners.

a. Being positive for HPV16/18/31/33/35/39/45/51/52/56/58 or HPV59.

b. Calculated using Chi-square tests.

c. High educational level included school of higher general secondary education, pre-university education, university of applied sciences, and university. Low/middle educational level included all other levels of education.

d. Based on information of the STI clinic visit.

e. Inconsistent included reporting never, rarely and "sometimes I do, sometimes I do not" condom use. Consistent included reporting often or always condom use. 
Supplementary Table 5.2. Characteristics over time and relation with hrHPV positivity among heterosexual men.

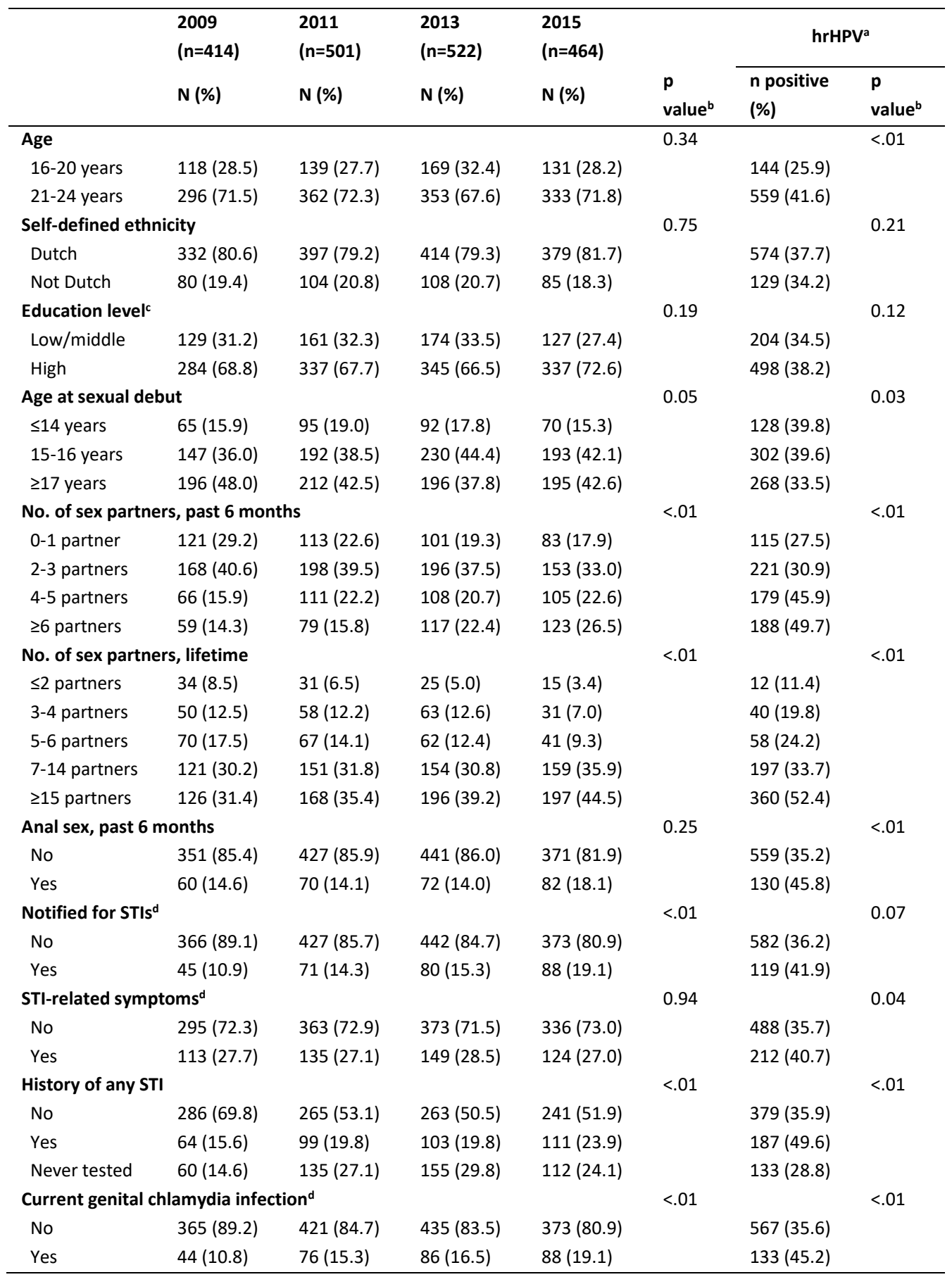


Supplementary Table 5.2. (Continued).

\begin{tabular}{|c|c|c|c|c|c|c|c|}
\hline & \multirow{2}{*}{$\begin{array}{l}2009 \\
(n=414) \\
N(\%)\end{array}$} & \multirow{2}{*}{$\begin{array}{l}2011 \\
(n=501) \\
N(\%)\end{array}$} & \multirow{2}{*}{$\begin{array}{l}2013 \\
(n=522) \\
N(\%)\end{array}$} & \multirow{2}{*}{$\begin{array}{l}2015 \\
(n=464) \\
N(\%)\end{array}$} & \multirow[b]{2}{*}{$\begin{array}{l}\mathbf{p} \\
\text { value }^{\mathbf{b}}\end{array}$} & \multicolumn{2}{|c|}{ hrHPV } \\
\hline & & & & & & $\begin{array}{l}\text { n positive } \\
(\%)\end{array}$ & $\begin{array}{l}\mathbf{p} \\
\text { value }^{\mathrm{b}}\end{array}$ \\
\hline \multicolumn{4}{|l|}{ Steady partner } & & \multirow[t]{4}{*}{$<.01$} & & 0.02 \\
\hline No & 206 (51.1) & $253(53.0)$ & $284(55.8)$ & $294(65.8)$ & & 383 (36.9) & \\
\hline Yes, for $0-6 m$ & $108(26.8)$ & $134(28.1)$ & $142(27.9)$ & $91(20.4)$ & & $192(40.4)$ & \\
\hline Yes, for $\geq 6 m$ & $89(22.1)$ & 90 (18.9) & $83(16.3)$ & 62 (13.9) & & 100 (30.9) & \\
\hline \multicolumn{5}{|c|}{ Condom use with casual partners, past 6 months ${ }^{e}$} & \multirow[t]{4}{*}{$<.01$} & & $<.01$ \\
\hline Inconsistent & $146(35.4)$ & $211(42.4)$ & $240(46.1)$ & $254(55.0)$ & & $345(40.5)$ & \\
\hline Consistent & $152(36.8)$ & 179 (35.9) & $181(34.7)$ & $146(31.6)$ & & 249 (37.8) & \\
\hline $\begin{array}{l}\text { No casual } \\
\text { partners }\end{array}$ & $115(27.8)$ & $108(21.7)$ & $100(19.2)$ & $62(13.4)$ & & 105 (27.3) & \\
\hline \multicolumn{8}{|c|}{$\begin{array}{l}\text { Totals vary because of missing values. Sexual risk behavior variables that were associated with PASSYON study year ( } \mathrm{p}<.05) \text { and } \\
\text { hrHPV ( }<<.05 \text { ) were considered in a computerized stepwise selection model, to select confounders to adjust for. The variables that } \\
\text { were selected in the stepwise model were: lifetime sex partners, history of any STI, and steady partner. } \\
\text { a. Being positive for HPV16/18/31/33/35/39/45/51/52/56/58 or HPV59. } \\
\text { b. Calculated using Chi-square tests. } \\
\text { c. High educational level included school of higher general secondary education, pre-university education, university of applied } \\
\text { sciences, and university. Low/middle educational level included all other levels of education. } \\
\text { d. Based on information of the STI clinic visit. } \\
\text { e. Inconsistent included reporting never, rarely and "sometimes I do, sometimes I do not" condom use. Consistent included reporting } \\
\text { often or always condom use. }\end{array}$} \\
\hline
\end{tabular}


Supplementary Table 5.3. Characteristics over time and relation with hrHPV positivity among unvaccinated women.

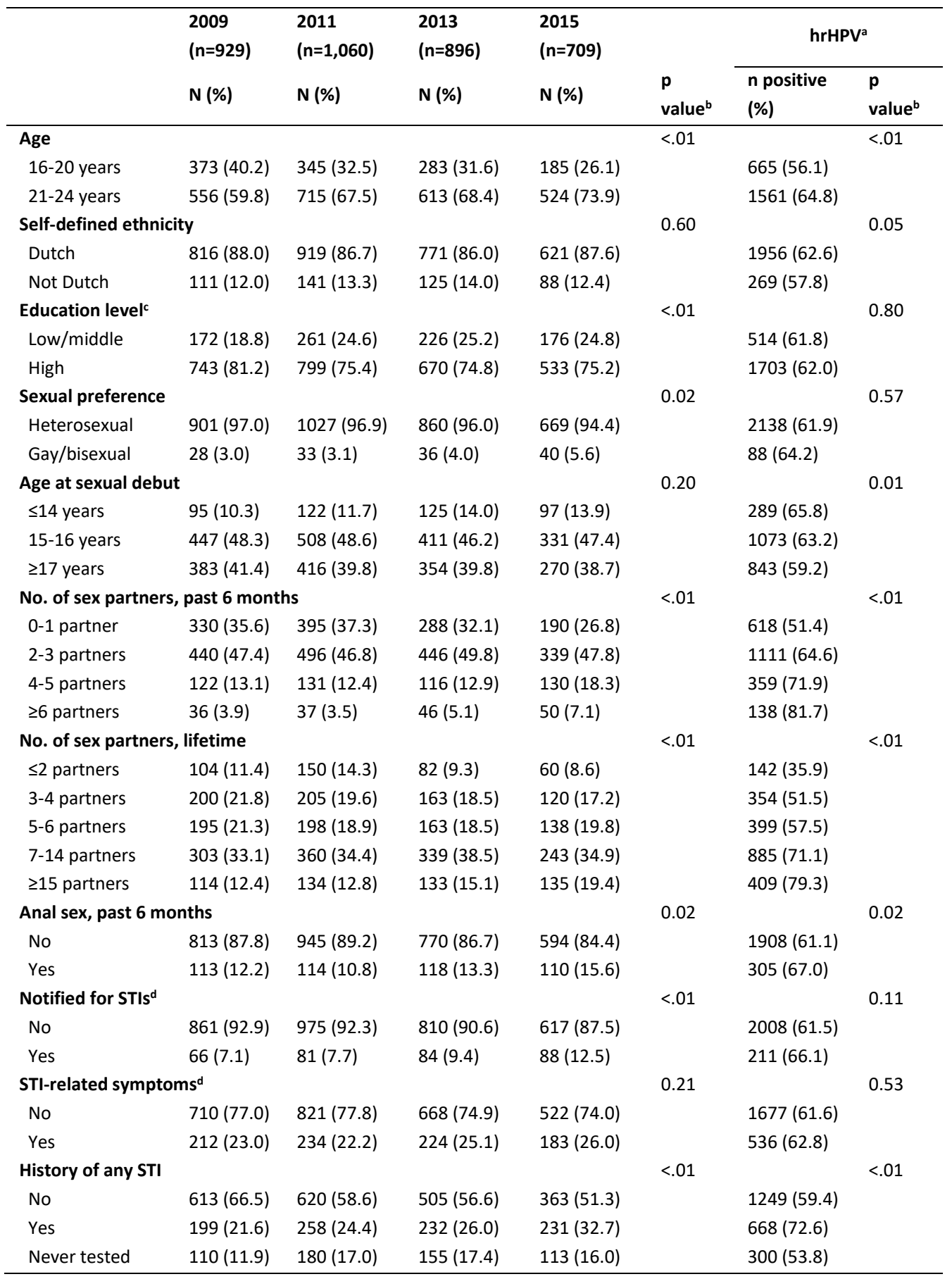


Supplementary Table 5.3. (Continued).

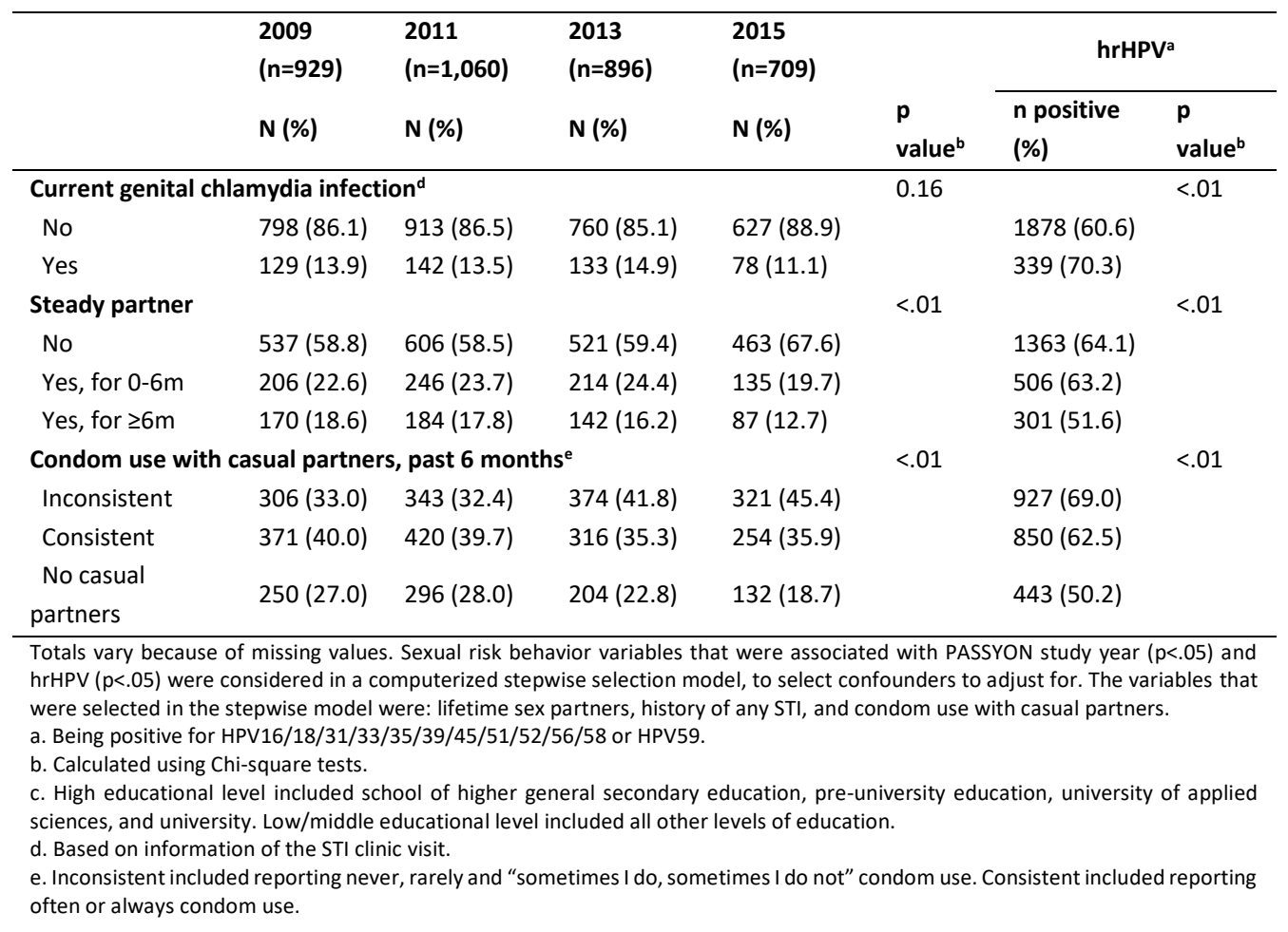


Supplementary Table 4.5. Comparing post-vaccination HPV prevalence with prevaccination prevalence (2009) and assessing the trend among heterosexual men, excluding the men who reported to be HPV vaccinated.

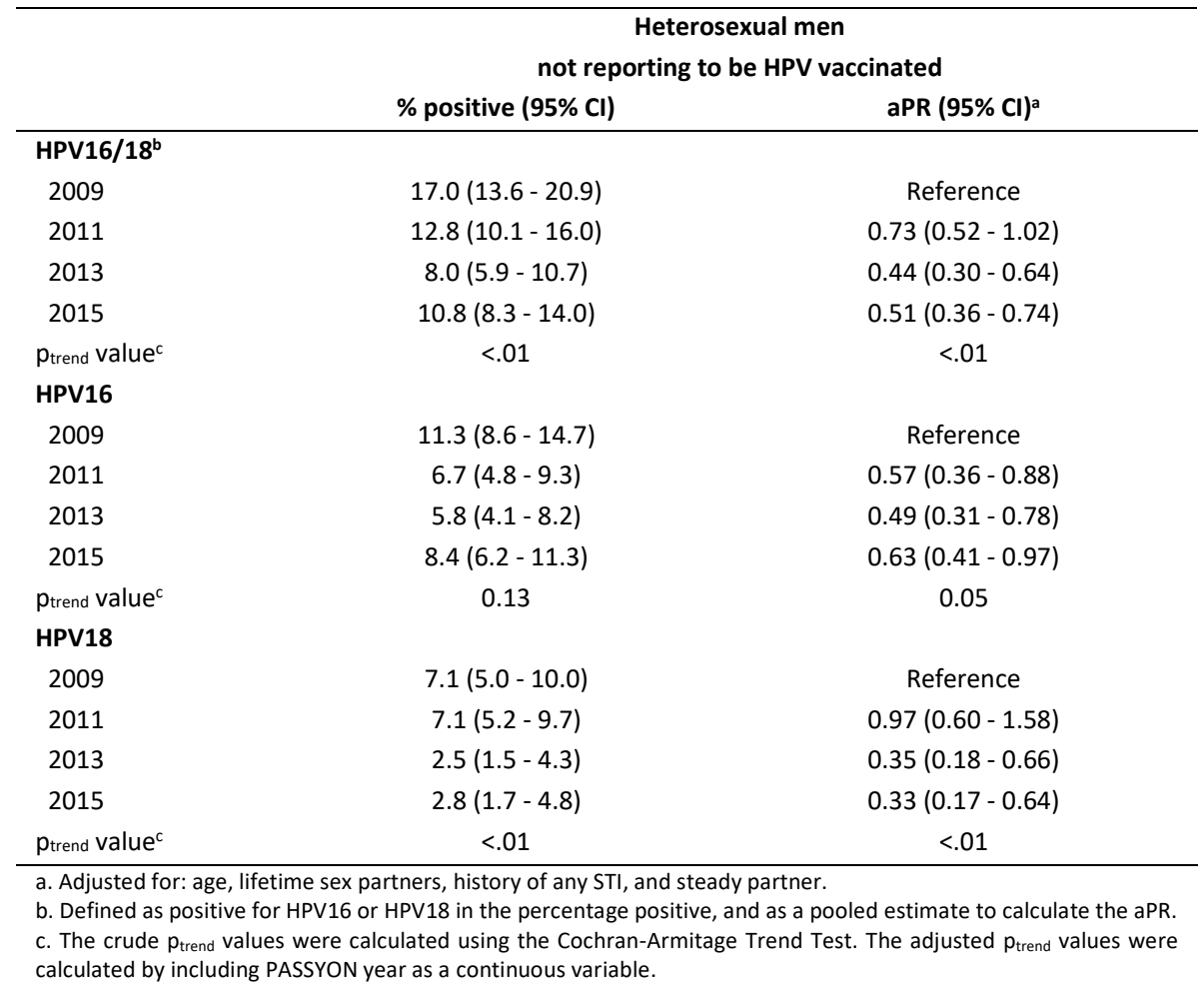




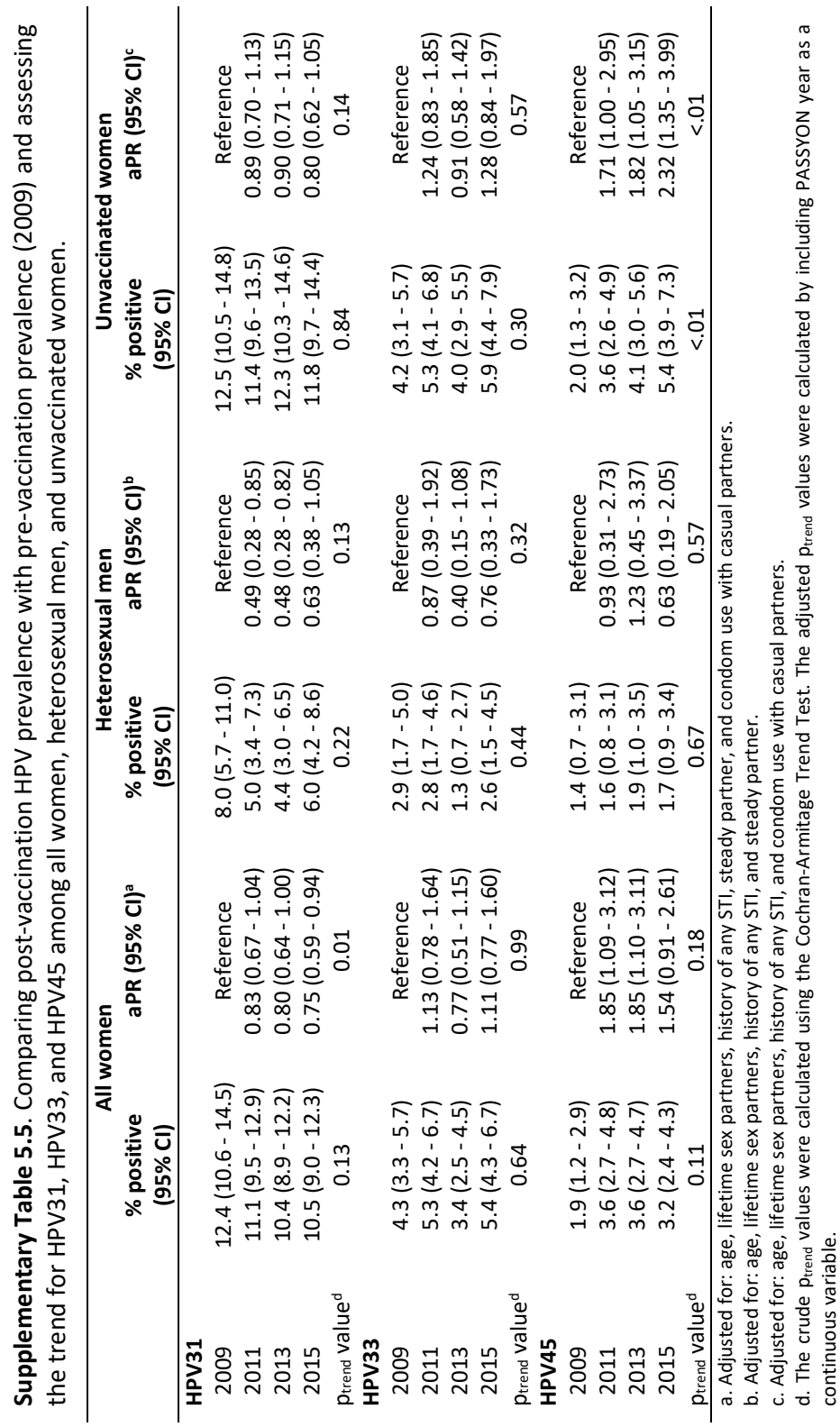




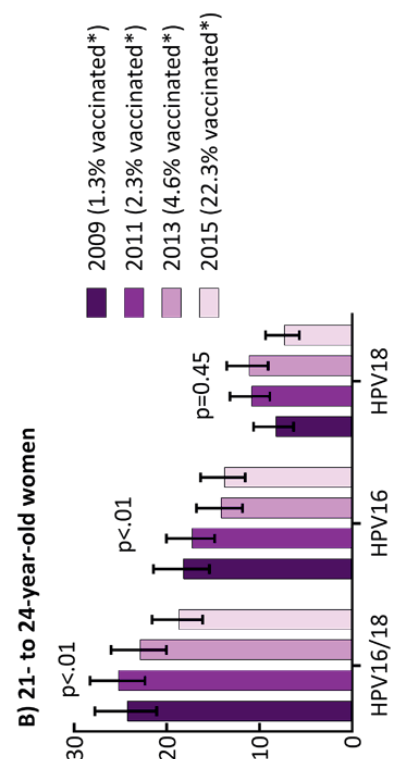

(\%) әэиәреләд

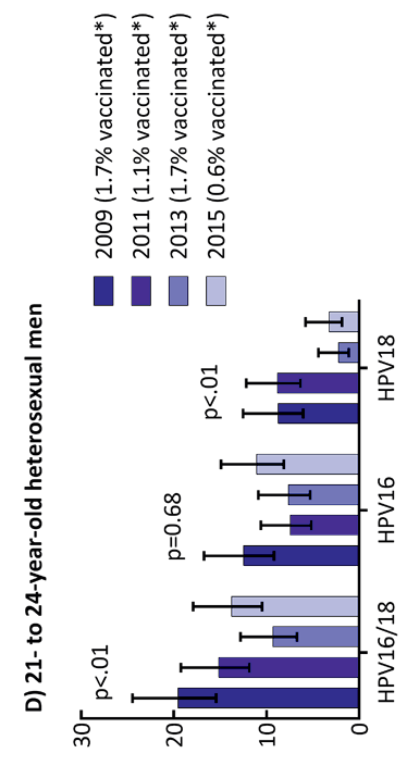

(\%) әэиәрелдл

(\%) әэиәреләג
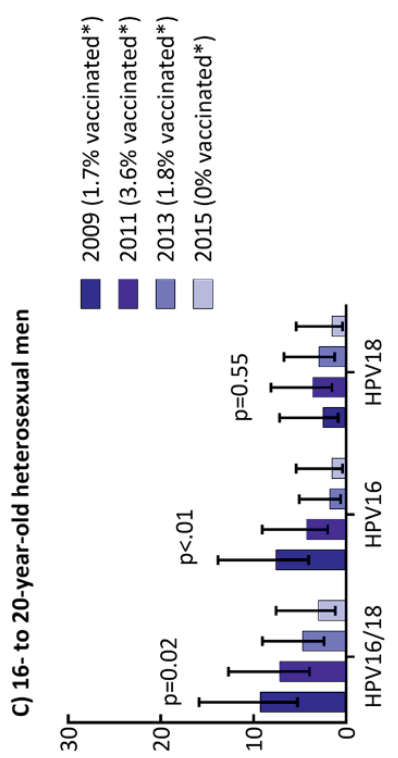

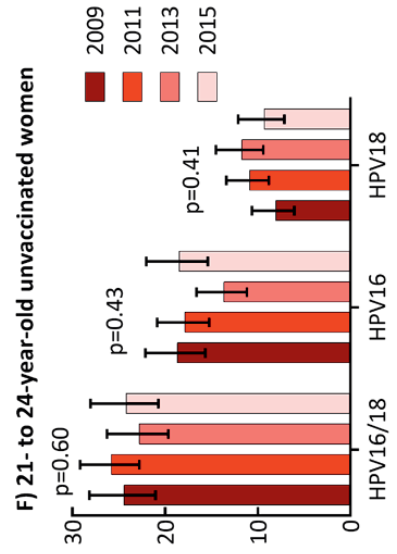

(\%) әэиәрләдd

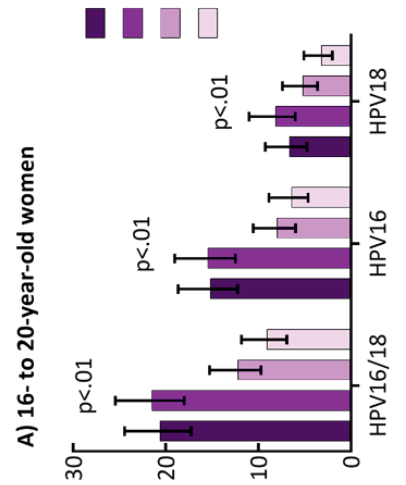

(\%) әэиәреләдd

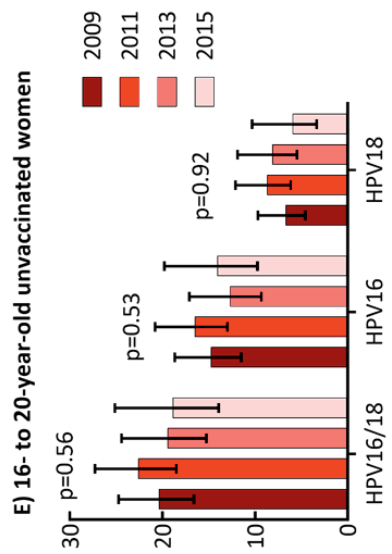

(\%) әэиәреләд

Supplementary Figure 5.1. Prevalence of HPV types 16 or 18, HPV16, and HPV18 over time and $p$ vales for the crude trend test, among: A) 16- to 20-year-old women; B) 21- to 24-year-old women; C) 16- to 20-year-old heterosexual men; D) 21- to 24-year-old heterosexual men; E) 16- to 20-year-old unvaccinated women; F) 21- to 24-year-old unvaccinated women.

Note: The $p$ value presents the Cochran-Armitage Trend Test. * Percentage of women and heterosexual men who reported to be vaccinated at least once. 


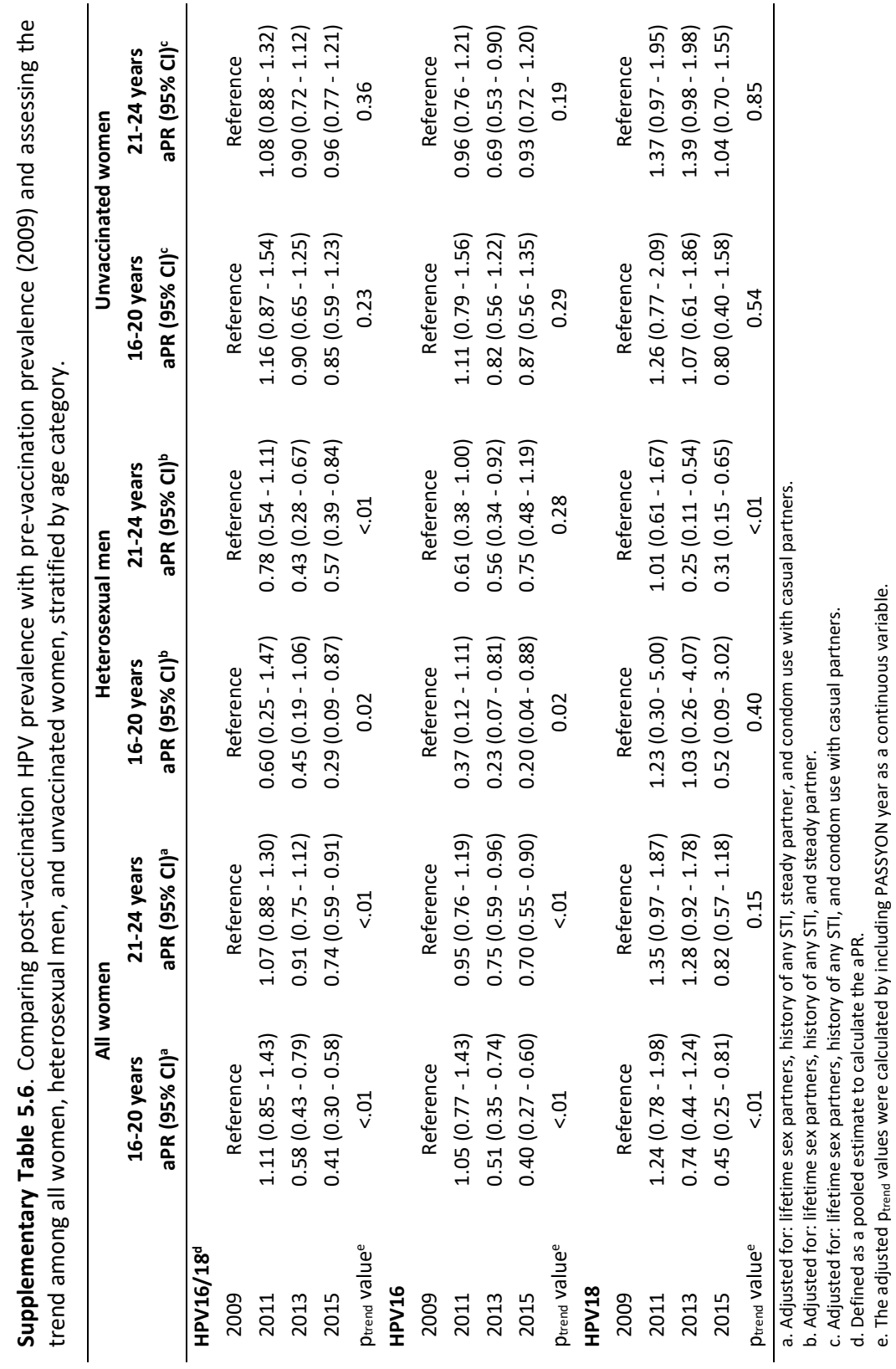





\title{
Chapter 6
}

\section{Population impact of girls-only HPV16/18 vaccination in the Netherlands: Cross-protective and second-order herd effects}

\author{
Joske Hoes* \\ Petra J. Woestenberg* \\ Johannes A. Bogaards \\ Audrey J. King \\ Hester E. de Melker \\ Johannes Berkhof \\ Christian J. P. A. Hoebe \\ Marianne A. B. van der Sande \\ Birgit H. B. van Benthem
}




\section{Abstract}

Introduction: Human papillomavirus (HPV) vaccination programs achieve substantial population-level impact, with effects extending beyond protection of vaccinated individuals. We assessed trends in HPV prevalence up to 8 years post-vaccination among men and women in the Netherlands, where bivalent HPV (2vHPV) vaccination, targeting HPV16/18, has been offered to (pre)adolescent girls since 2009 with moderate vaccination coverage.

Methods: We used data from the PASSYON study, a survey initiated in 2009 (prevaccination) and repeated biennially among 16- to 24-year-old visitors of sexual health centers. We studied genital HPV positivity from 2009 to 2017 among women, heterosexual men, and unvaccinated women using Poisson GEE models, adjusted for individual- and population-level confounders. Trends were studied for 25 HPV types detected by the SPF10LiPA25 platform.

Results: 6,354 women (64.7\% self-reported unvaccinated) and 2,414 heterosexual men were included. Percentual declines in vaccine types HPV16/18 were observed for all women (12.6\% per year; 95\% confidence interval [CI] 10.6\%-14.5\%), heterosexual men (13.0\% per year; $95 \% \mathrm{Cl} 8.3 \%-17.5 \%$ ), and unvaccinated women (5.4\% per year; $95 \% \mathrm{Cl} 2.9 \%-7.8 \%$ ). We observed significant declines in HPV31 (all women and heterosexual men), HPV45 (all women), and in all high-risk HPV types pooled (all women and heterosexual men). Significant increases were observed for HPV56 (all women) and HPV52 (unvaccinated women).

Discussion: Our results provide evidence for first-order herd effects among heterosexual men against HPV16/18 and cross-protective HPV types. Additionally, we show second-order herd effects against vaccine types among unvaccinated women. These results are promising regarding population-level and clinical impact of girls-only $2 \mathrm{vHPV}$ vaccination in a country with moderate vaccine uptake. 


\section{Introduction}

Infections with human papillomavirus (HPV) are usually transient, however persistent infections may induce illness in the anogenital or oropharyngeal regions among men and women. Most common are anogenital warts (AGWs), but persistent infections with a highrisk HPV (hrHPV) type can also lead to various cancers [1]. From 2006 onwards, 3 prophylactic vaccines have been registered for prevention of HPV-related (pre)cancers, all targeting the most oncogenic hrHPV types 16 and 18. The Netherlands have included the bivalent HPV (2vHPV) vaccine, targeting HPV16/18, in their national immunization program (NIP). HPV16/18 vaccination is offered to 12 -year-old girls in the routine program since 2010, after a one-off catch-up campaign in 2009 for 12- to 16-year-old girls (birth cohorts 1993-1996) [2]. So far, vaccine uptake among vaccine-eligible girls has fluctuated between $46 \%$ and $61 \%$ per year between 2009 and 2017 [3].

Vaccine effectiveness (VE) of the 2VHPV vaccine against (persistent) infection with vaccine and cross-protective HPV types has been shown in vaccine recipients relative to controls [4, 5]. However, the population impact of HPV vaccination extends beyond direct protection of vaccinated individuals, as infection dynamics change through vaccination implementation. In countries achieving high coverage in girls-only quadrivalent HPV vaccination programs, also targeting low-risk HPV (IrHPV) types 6 and 11, indirect benefits were evident early on through reduced prevalence of AGWs in unvaccinated young men [6]. Additionally, declining hrHPV prevalence in young vaccinated women was sufficient to provide herd protection to unvaccinated women within 6 to 7 years after initiation of girlsonly HPV vaccination in settings with $>80 \%$ coverage $[7,8]$. Herd effects among unvaccinated women are mainly derived from unvaccinated, but indirectly protected, heterosexual men. However, men have been underrepresented in studies assessing population trends in HPV prevalence over time since vaccine introduction [9].

Previously, we demonstrated herd effects for vaccine types HPV16/18 among heterosexual men 6 years after introduction of girls-only HPV16/18 vaccination in the Netherlands [10]. Herd effects among unvaccinated women were not yet observed within 6 years post-vaccination, presumably due to the moderate vaccine uptake in the Netherlands. In a girls-only vaccination program, herd protection of unvaccinated women constitutes a second-order effect, and is strongly determined by vaccination coverage [11]. As we observed herd effects among heterosexual men within 6 years post-vaccination, we hypothesized that herd effects among unvaccinated women in the Netherlands should also become measurable with prolonged monitoring. Continued monitoring of trends in typespecific HPV prevalence over time is also relevant for detection of type-replacement, a still unresolved possibility in the wake of HPV vaccination [12]. 
To further assess the population impact of the girls-only HPV16/18 vaccination program in the Netherlands on post-vaccination trends in vaccine and non-vaccine HPV types, we updated and expanded our previous analyses. Here, we present trends in HPV positivity of $25 \mathrm{HPV}$ types from pre-vaccination up to 8 years post-vaccination among 16 - to 24 -year-old women and heterosexual men visiting sexual health centers (SHCs) throughout the Netherlands.

\section{Methods}

\section{Study design and population}

For this updated analysis, we used data from the PASSYON (PApillomavirus Surveillance among STI clinic YOungsters in the Netherlands) study: a biennial cross-sectional survey to assess the HPV prevalence among young visitors to SHCs [10]. In the Netherlands, SHCs offer sexually transmitted infection (STI) testing to those with increased risk, including $\leq 24$ yearold individuals. The study design has been described previously [13]. Briefly, the PASSYON study was initiated in 2009 (pre-vaccination) and repeated in 2011, 2013, 2015, and 2017 in SHCs throughout the country (Figure 6.1). Male and female SHC visitors $16-24$ years of age were approached for participation and asked to collect a genital self-swab (Copan Diagnostics, Italy). Women were instructed to take a cervicovaginal sample, while men took a penile sample. A questionnaire on sexual risk behavior, demographics, and vaccination status was collected, which was supplemented with information from the regular SHC consult. The Medical Ethical Committee of the University of Utrecht, the Netherlands, provided a waiver for full medical ethical review (protocol number 08/397). Data were obtained using a unique code per person and all participants gave informed consent.

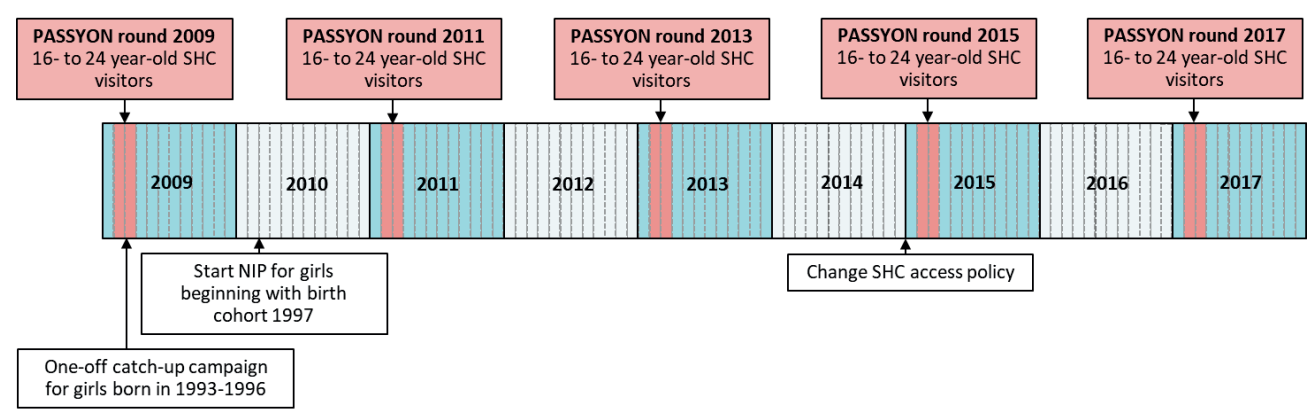

Figure 6.1. HPV vaccination in the Netherlands and the PASSYON study design. 


\section{Laboratory analyses}

HPV laboratory testing was conducted similarly across all study years [13]. Briefly, DNA was extracted using the MagnaPure platform (Total Nucleic Acid Isolation Kit, Roche, the Netherlands) and HPV DNA was amplified using the SPF10 primer set and detected using the DNA enzyme-linked immunoassay (HPV-DEIA, DDL Diagnostics Laboratory, the Netherlands). Positive samples were genotyped with line-probe assay (HPV-LiPA25, DDL Diagnostics Laboratory, the Netherlands), which is able to detect hrHPV types $16,18,31$, 33, 35, 39, 45, 51, 52, 56, 58, 59, and IrHPV types 6, 11, 34, 40, 42, 43, 44, 53, 54, 66, 70, 74. Also HPV68, 73, and 97 can be detected, but these types cannot be distinguished from each other and are therefore classified as HPV68*.

\section{Statistical analyses}

Trends in HPV positivity were studied for all women and self-reported heterosexual men. To study second-order herd effects, we additionally analyzed trends in self-reported unvaccinated women.

The crude prevalence of hrHPV and IrHPV types was calculated for each study year, stratified by gender and vaccination status. Trends in type-specific HPV prevalence over time were assessed using generalized estimation equation (GEE) Poisson models with a log link function and robust error variance. The incorporation of an exchangeable correlation structure accounted for dependency of HPV types within individuals and ensured efficient estimation of regression coefficients. PASSYON year was added as a continuous variable to the model, resulting in a linear trend of HPV prevalence over time on a log scale. Percentual changes in HPV prevalence per year were estimated by exponentiating the (adjusted) regression coefficient for each HPV type.

To select possible confounding variables in the estimation of HPV prevalence trends, we first examined the study population over time regarding participant characteristics. Using $\chi^{2}$ tests, we checked whether characteristics were comparable between different study years. Next, we studied the association between HPV positivity (any type) and participant characteristics, again using $\chi^{2}$ tests. Characteristics associated with study year and with HPV positivity were included as explanatory variables in a logistic regression stepwise selection model (with $\mathrm{p}<.05$ as entry and stay criteria), using HPV positivity as outcome. Variables included in the final model were used for adjustment in the Poisson GEE models. This process was performed separately for all women, heterosexual men, and unvaccinated women.

Next to individual-level confounders indicated by the selection models, trends in HPV prevalence were adjusted for age group (16-20 vs 21-24 years) and for changes in SHC access policy (population-level confounder). Due to funding restrictions from 2015 onwards, SHCs became stricter in prioritizing individuals at high risk for STIs, which could 
have resulted in a study population at systematically increased risk for HPV infection starting from 2015 [14]. As we assumed we were unable to fully adjust for this by only including changes over time in the known variables [10], we adjusted for policy change by including a categorical variable indicating the policy change from 2015 onwards.

Additionally, we estimated pooled (adjusted) trends in HPV positivity over time. Pooled estimates were obtained as a weighted average of type-specific trends in the GEE Poisson models. Pooled trends were estimated for vaccine types (HPV16/18), hrHPV types of the nonavalent (9vHPV) vaccine (HPV16/18/31/33/45/52/58), all hrHPV types (HPV16/18/31/ $33 / 35 / 39 / 45 / 51 / 52 / 56 / 58 / 59)$ and all types measured in the SPF10-DEIA-LiPA25 assay. As the impact of vaccination on the overall HPV prevalence among 16- to 24-year-olds was likely to be still very low in 2011 [15], we performed sensitivity analyses pooling data from PASSYON years 2009 and 2011 to create more stable baseline measurements.

All analyses were performed using SAS version 9.4 (SAS Institute Inc., Cary, NC). We performed complete case analyses, as none of the variables had more than $5 \%$ missing.

\section{Results}

We included a total of 6,354 women (1,574 vaccinated with at least one dose, 4,111 unvaccinated, 669 unknown; all self-reported) and 2,414 heterosexual men who all provided a genital swab; the study population was 1,524 in 2009, 1,775 in 2011, 1,816 in $2013,1,782$ in 2015 , and 1,871 in 2017 . The percentage of women reporting to be vaccinated increased over the years; $2.3 \%$ in $2009,6.4 \%$ in $2011,19.2 \%$ in $2013,36.7 \%$ in 2015 , and $54.1 \%$ in 2017 . In total, $13.2 \%$ of the vaccinated women were vaccinated within the regular program.

Characteristics of the total study population are presented in Table 6.1. The association between characteristics and study year and the association between characteristics and HPV positivity are given separately for all women, heterosexual men, and unvaccinated women in Supplementary Tables 6.1, 6.2, 6.3. In general, sexual risk behavior increased over time in all groups. 
Table 6.1. Characteristics of the study population.

\begin{tabular}{|c|c|c|c|}
\hline & $\begin{array}{l}\text { All women } \\
n(\%)\end{array}$ & $\begin{array}{l}\text { Heterosexual men } \\
n(\%)\end{array}$ & $\begin{array}{l}\text { Unvaccinated women } \\
\mathrm{n}(\%)\end{array}$ \\
\hline Total & 6354 & 2414 & 4111 \\
\hline \multicolumn{4}{|l|}{ Age } \\
\hline $16-20$ years & $2478(39.0)$ & $691(28.6)$ & $1336(32.5)$ \\
\hline 21-24 years & $3876(61.0)$ & $1723(71.4)$ & $2775(67.5)$ \\
\hline \multicolumn{4}{|c|}{ Self-defined ethnicity } \\
\hline Dutch & $5514(86.8)$ & $1933(80.1)$ & $3579(87.1)$ \\
\hline Not Dutch & $837(13.2)$ & $479(19.9)$ & $530(12.9)$ \\
\hline \multicolumn{4}{|l|}{ Education level $^{a}$} \\
\hline Low/middle & $1550(24.5)$ & $708(29.4)$ & $964(23.5)$ \\
\hline High & $4773(75.5)$ & $1699(70.6)$ & $3133(76.5)$ \\
\hline \multicolumn{4}{|c|}{ Sexual preference } \\
\hline Heterosexual & $6070(95.5)$ & $2414(100.0)$ & $3940(95.8)$ \\
\hline Gay or bisexual & $284(4.5)$ & $0(0.0)$ & $171(4.2)$ \\
\hline \multicolumn{4}{|c|}{ Age at sexual debut } \\
\hline$\leq 14$ years & $813(13.0)$ & 405 (16.9) & $504(12.4)$ \\
\hline $15-16$ years & $3036(48.3)$ & $961(40.2)$ & $1934(47.5)$ \\
\hline$\geq 17$ years & $2428(38.7)$ & $1024(42.9)$ & $1630(40.1)$ \\
\hline \multicolumn{4}{|c|}{ No. of sex partners, past 6 months } \\
\hline $0-1$ partners & 1960 (30.9) & $499(20.7)$ & $1342(32.7)$ \\
\hline 2-3 partners & $3047(48.0)$ & $876(36.3)$ & $1956(47.6)$ \\
\hline 4-5 partners & $947(14.9)$ & $510(21.1)$ & $591(14.4)$ \\
\hline$\geq 6$ partners & $394(6.2)$ & 529 (21.9) & $219(5.3)$ \\
\hline \multicolumn{4}{|c|}{ No. of sex partners, lifetime } \\
\hline$\leq 2$ partners & $682(10.9)$ & $130(5.6)$ & $438(10.8)$ \\
\hline 3-4 partners & 1176 (18.9) & $245(10.6)$ & $770(19.0)$ \\
\hline 5-6 partners & $1220(19.6)$ & $284(12.3)$ & 789 (19.5) \\
\hline 7-14 partners & $2196(35.2)$ & $749(32.4)$ & $1417(35.0)$ \\
\hline$\geq 15$ partners & $962(15.4)$ & $903(39.1)$ & 635 (15.7) \\
\hline \multicolumn{4}{|c|}{ Anal sex, past 6 months } \\
\hline No & $5527(87.4)$ & $2021(84.7)$ & $3568(87.2)$ \\
\hline Yes & $796(12.6)$ & $365(15.3)$ & $526(12.8)$ \\
\hline \multicolumn{4}{|l|}{ Notified for STIs ${ }^{b}$} \\
\hline No & $5630(89.1)$ & $1992(82.9)$ & $3684(90.0)$ \\
\hline Yes & $688(10.9)$ & $410(17.1)$ & $408(10.0)$ \\
\hline \multicolumn{4}{|c|}{ STI-related symptoms ${ }^{b}$} \\
\hline No & $4818(76.4)$ & $1742(72.7)$ & $3100(75.9)$ \\
\hline Yes & $1491(23.6)$ & $655(27.3)$ & $984(24.1)$ \\
\hline
\end{tabular}


Table 6.1. (Continued).

\begin{tabular}{|c|c|c|c|}
\hline & $\begin{array}{l}\text { All women } \\
n(\%)\end{array}$ & $\begin{array}{l}\text { Heterosexual men } \\
n(\%)\end{array}$ & $\begin{array}{l}\text { Unvaccinated women } \\
\mathrm{n}(\%)\end{array}$ \\
\hline \multicolumn{4}{|l|}{ STI-related symptoms $^{\mathrm{b}}$} \\
\hline No & $4818(76.4)$ & $1742(72.7)$ & $3100(75.9)$ \\
\hline Yes & $1491(23.6)$ & $655(27.3)$ & $984(24.1)$ \\
\hline \multicolumn{4}{|l|}{ History of any STI } \\
\hline No & $3549(56.2)$ & $1298(54.0)$ & $2361(57.6)$ \\
\hline Yes & $1683(26.6)$ & $508(21.1)$ & $1087(26.6)$ \\
\hline Never tested & $1089(17.2)$ & $598(24.9)$ & $648(15.8)$ \\
\hline \multicolumn{4}{|c|}{ Current genital chlamydia infection ${ }^{\mathrm{b}}$} \\
\hline No & $5403(85.5)$ & $2010(83.8)$ & $3534(86.4)$ \\
\hline Yes & $914(14.5)$ & $388(16.2)$ & $557(13.6)$ \\
\hline \multicolumn{4}{|l|}{ Steady partner } \\
\hline No & $3883(62.6)$ & $1359(58.3)$ & $2470(61.5)$ \\
\hline Yes, for $0-6$ months & $1366(22.0)$ & $584(25.1)$ & $908(22.6)$ \\
\hline Yes, $\geq 6$ months & $959(15.4)$ & $386(16.6)$ & $641(15.9)$ \\
\hline \multicolumn{4}{|c|}{ Condom use with casual partners, past 6 months $^{c}$} \\
\hline Inconsistent & $2624(41.5)$ & $1139(47.6)$ & $1601(39.1)$ \\
\hline Consistent & $2243(35.5)$ & $810(33.8)$ & $1525(37.2)$ \\
\hline No casual partners & $1455(23.0)$ & $445(18.6)$ & $972(23.7)$ \\
\hline
\end{tabular}

Totals vary because of missing values.

a. High educational level included school of higher general secondary education, pre-university education, university of applied sciences, and university. Low/middle educational level included all other levels of education.

b. Based on information of the sexual health center visit.

c. Inconsistent included reporting never, rarely and "sometimes I do, sometimes I do not" condom use. Consistent included reporting often or always condom use.

Figure 6.2 displays the crude HPV prevalence over time for all women, heterosexual men, and unvaccinated women. HPV prevalence was positively affected by the SHC policy change from 2015 onwards. After adjustment for age and changes in individual-level characteristics over time, this policy change was predicted to have elevated the HPV type-specific positivity by $9 \%$ among women and up to $30 \%$ among heterosexual men in trend analyses. Overall, after adjustment for age and selected participant characteristics, and more so after adjustment for policy change, declining trends in HPV prevalence over time became stronger, while increasing trends became weaker (Supplementary Table 6.4). 


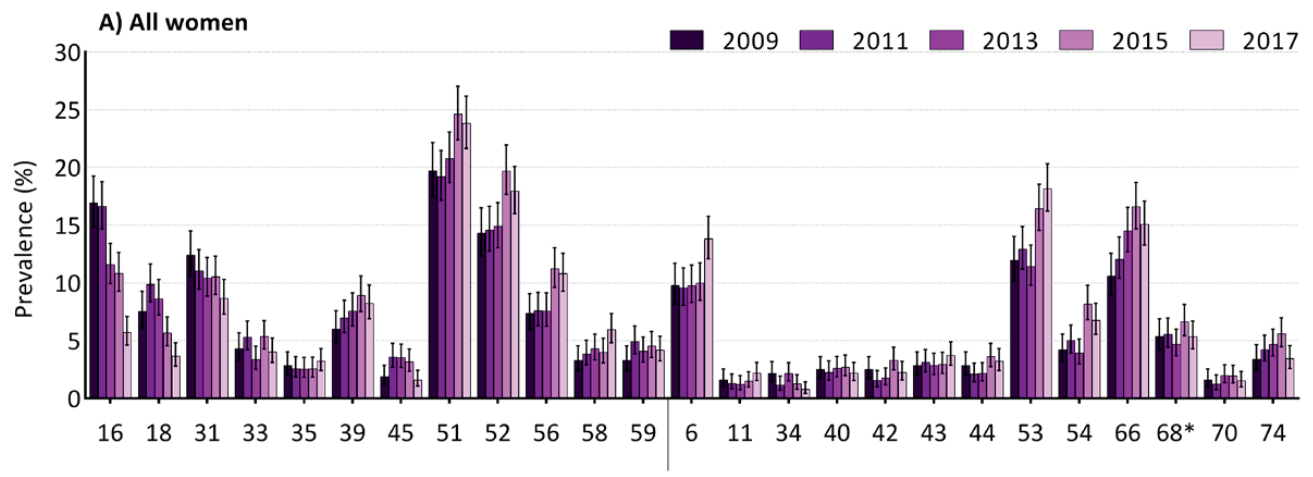

B) Heterosexual men

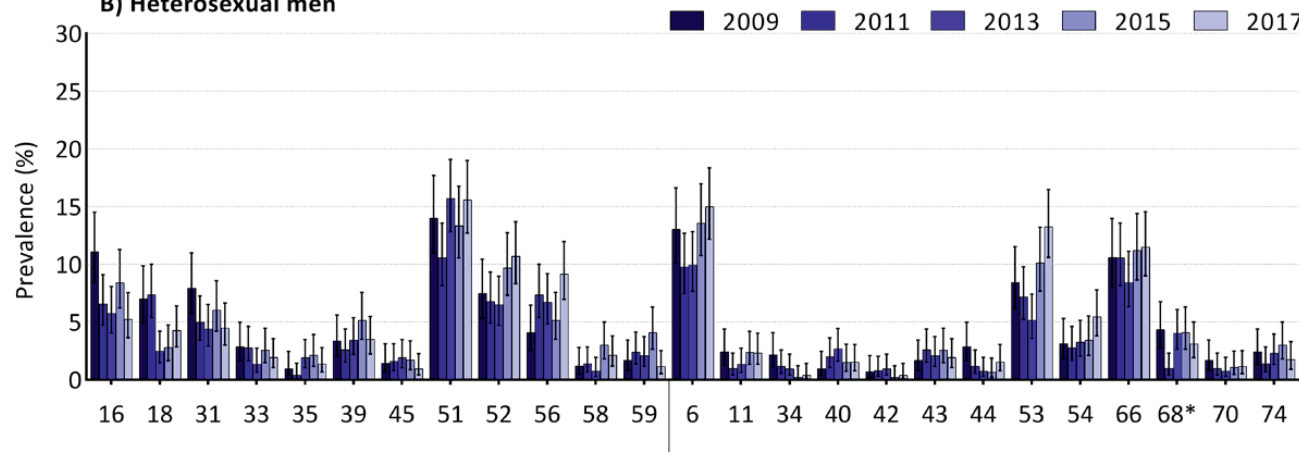

C) Unvaccinated women

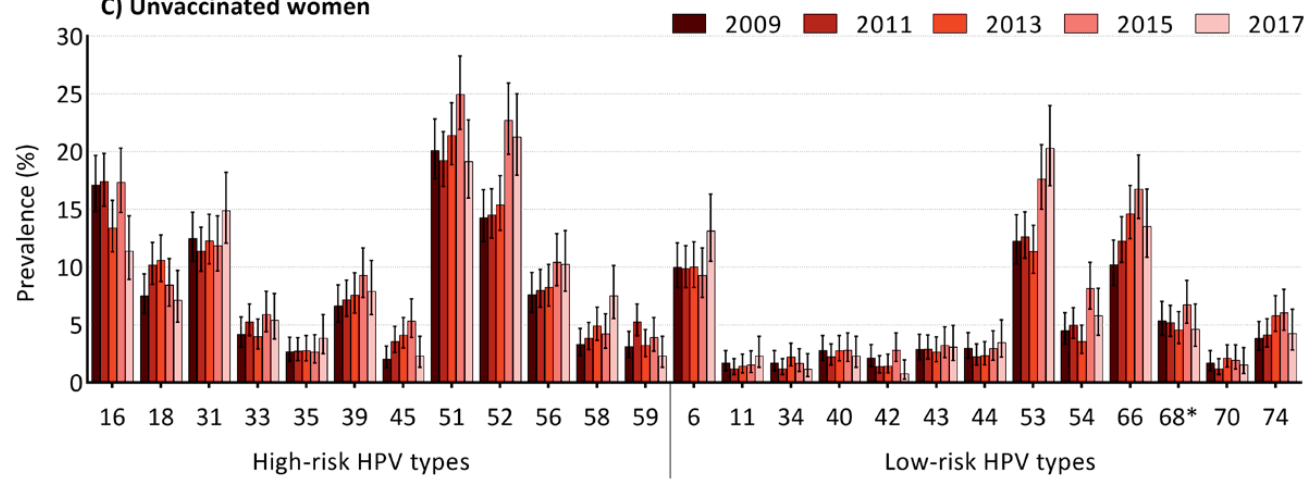

Figure 6.2. HPV prevalence for the different years of the PASSYON study among A) all women; B) heterosexual men; $C$ ) unvaccinated women.

Note: HPV68* also includes HPV73 and HPV97. From 2015 onwards the access policy at the sexual health centers had changed, leading to prioritizing of individuals at high risk for sexually transmitted infections. 
In the final adjusted GEE models, decreasing trends in both vaccine types HPV16 and HPV18 were estimated for women, heterosexual men, and unvaccinated women separately (Figure 6.3). The pooled percentual decline in HPV16/18 prevalence per year was $12.6 \%$ (95\% confidence interval [Cl] 10.6\%-14.5\%) among all women, $13.0 \%$ (95\% Cl 8.3\%-17.5\%) among heterosexual men, and $5.4 \%(95 \% \mathrm{Cl}$ 2.9\%-7.8\%) among unvaccinated women (Table 6.2). Declining trends were also observed for cross-protective types. We estimated significantly declining trends in the prevalence of HPV31, with a $6.8 \%$ annual decline $(p<.001)$ among all women and a $9.7 \%$ annual decline $(p=0.005)$ among heterosexual men, and in the prevalence of HPV45, with a $4.9 \%$ annual decline $(p=0.036)$ among all women. The decline in HPV45 prevalence of $10.4 \%$ annually among heterosexual men was borderline nonsignificant $(p=0.065)$. Other significantly declining trends in adjusted analyses were seen for HPV59 (unvaccinated women), for HPV34 (all women and heterosexual men), and for HPV44 (heterosexual men).

Also increasing trends in HPV prevalence were observed. In adjusted GEE models the prevalence of HPV56 and HPV54 increased among all women, and the prevalence of HPV52 increased among unvaccinated women. A complete overview of the trends for all $25 \mathrm{HPV}$ types is provided in Figure 6.3.

Pooling the trend of hrHPV types of the 9vHPV vaccine and all hrHPV types, resulted in a $5.7 \%$ and $3.0 \%$ annual decline among women and in a $7.8 \%$ and $5.3 \%$ annual decline among heterosexual men (Table 6.2). The pooled trend of all measured HPV types including hrHPV and IrHPV types was declining among women and heterosexual men ( $1.6 \%$ and $4.1 \%$ annual decline respectively). Among unvaccinated women, none of the pooled trends were statistically significant.

Sensitivity analyses in which the first 2 PASSYON years were pooled and considered as 1 baseline measurement yielded comparable results regarding adjusted trends for HPV vaccine types. Some type-specific trends became more pronounced, such as the decline of HVP45/33 among all women, while others were attenuated, such as the decrease in HPV31 among heterosexual men. The increasing trend in HPV56 among all women and in HPV52 among unvaccinated women were no longer statistically significant (Supplementary Table 6.5). 
A) All women

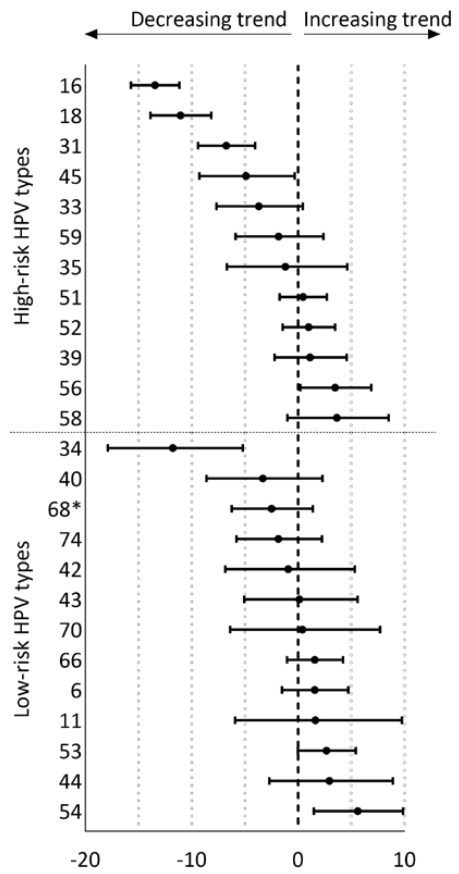

Percentual change in prevalence/year
B) Heterosexual men

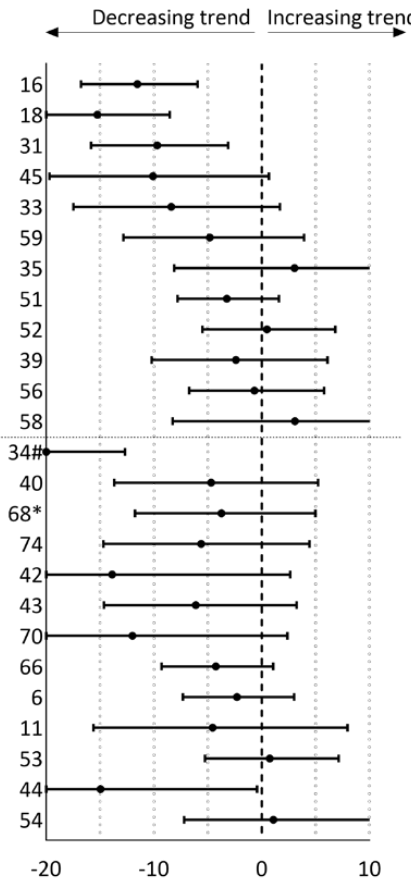

Percentual change in prevalence/year
C) Unvaccinated women

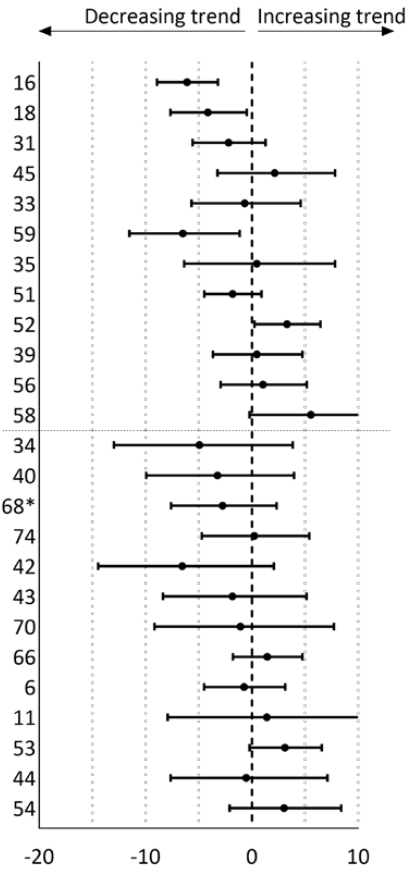

Percentual change in prevalence/year

Figure 6.3. Percentual change in prevalence of hrHPV and IrHPV types per year, among A) all women; B) heterosexual men; C) unvaccinated women.

Note: Percentual change in prevalence per year was calculated by exponentiating the adjusted regression coefficients of study year, which was added as a continuous variable in GEE analyses. For the exact percentual changes per year see Supplementary Table 6.4. HPV68* also includes HPV73 and HPV97. \#: point estimate for HPV34 among heterosexual men was $-26 \%$. Regression coefficients for all women were adjusted for age, policy change at the SHC, number of lifetime sex partners, history of any STI, steady partner, notified for STIs, number of sex partners in the past 6 months, and condom use with casual partners. Regression coefficients for heterosexual men were adjusted for age, policy change at the SHC, number of lifetime sex partners, and history of any STI. Regression coefficients for the unvaccinated women were adjusted for age, policy change at the SHC, number of lifetime sex partners, history of any STI, notified for STIS, number of sex partners in the past 6 months, and condom use with casual partners.

Table 6.2. Pooled trends in percentual change in HPV prevalence per year among all women, heterosexual men, and unvaccinated women.

\begin{tabular}{|c|c|c|c|}
\hline & $\begin{array}{l}\text { All women } \\
\%(95 \% \mathrm{Cl})\end{array}$ & $\begin{array}{l}\text { Heterosexual men } \\
\%(95 \% \mathrm{Cl})\end{array}$ & $\begin{array}{l}\text { Unvaccinated women } \\
\%(95 \% \mathrm{Cl})\end{array}$ \\
\hline $2 \mathrm{vHPV}$ types $^{\mathrm{a}}$ & $-12.58(-14.53$ to -10.59$)$ & $-13.04(-17.54$ to -8.30$)$ & $-5.38(-7.84$ to -2.87$)$ \\
\hline $9 \mathrm{v}$ hrHPV types ${ }^{\mathrm{b}}$ & $-5.73(-7.42$ to -4.02$)$ & $-7.82(-11.81$ to -3.64$)$ & $-1.28(-3.40$ to 0.87$)$ \\
\hline All hrHPV types ${ }^{c}$ & $-3.02(-4.61$ to -1.42$)$ & $-5.29(-8.96$ to -1.48$)$ & $-1.29(-3.22$ to 0.67$)$ \\
\hline All hr- and IrHPV types ${ }^{d}$ & $-1.59(-3.13$ to -0.03$)$ & $-4.10(-7.69$ to -0.38$)$ & $-0.67(-2.53$ to 1.22$)$ \\
\hline
\end{tabular}

Pooled trends were obtained as a weighted average of the type-specific trends in the GEE Poisson models. Percentual change in prevalence per year was calculated by exponentiating the adjusted regression coefficients of study year.

a. Including HPV types 16, 18.

b. Including HPV types $16,18,31,33,45,52,58$.

c. Including HPV types 16, 18, 31, 33, 45, 52, 58, 35, 39, 51, 56, 59.

d. Including HPV types 16, 18, 31, 33, 45, 52, 58, 35, 39, 51, 56, 59, 6, 11, 34, 40, 42, 43, 44, 53, 54, 66, 70, 74, 68/73/97. 


\section{Discussion}

We assessed trends in type-specific HPV prevalence for 25 HPV types up to 8 years after HPV16/18 vaccination implementation in the Netherlands. We demonstrated significant population impact of girls-only vaccination on vaccine-type HPV infection, with HPV16/18 prevalence declining each year by $13 \%$ among women and heterosexual men, and by $5.4 \%$ among unvaccinated women. We also demonstrated significant declines in HPV31 and HPV45 among women and heterosexual men, providing strong evidence that crossprotection of $2 \mathrm{vHPV}$ vaccination extends to unvaccinated individuals. Our results show that 2 VHPV vaccination induces herd effects against vaccine and cross-protective HPV types in a setting with moderate vaccination uptake.

Decreasing trends in HPV16/18 prevalence were observed among all groups. For women, this is partly explained by an increased proportion of vaccinated women over time who benefit from direct protection of HPV16/18 vaccination. In a previous analysis with data up to 6 years post-vaccination, we reported that heterosexual men already benefitted indirectly from this through herd protection [10]. This finding is reiterated in the current analyses with data up to 8 years post-vaccination. Additionally, we were now able to measure reductions in HPV16/18 prevalence among unvaccinated women, which constitutes a second-order effect that takes more time to develop. Meanwhile, no impact of vaccination implementation was observed among men having sex with men in the same period [16]. Our results are in line with observations from the United States, where vaccine coverage has been suboptimal as well (around 50\%) and herd effects among unvaccinated women were not yet present 3-6 years after vaccination, but became measurable 5-8 years after vaccination [17].

Cross-protection of the $2 \mathrm{vHPV}$ vaccine has been most clearly established for HPV types $31 / 33 / 45$ [18]. In line with this cross-protection, the current analyses showed significantly decreasing trends in HPV31/45 among all women, and in HPV31 among heterosexual men. Declining trends in HPV33 among all women and in HPV45 among heterosexual men were also pronounced, albeit non-significant. Declines in cross-protective HPV types were not yet observed in previous analyses [10]. Although natural fluctuation could occur over time, consistency of these results suggests that cross-protection of $2 \mathrm{vHPV}$ vaccination also leads to herd effects for these types, although second-order herd effects against cross-protective types remain to be demonstrated. Second-order herd effects against the pooled outcome HPV31/33/45 were seen in Scotland 7 years after 2vHPV vaccine introduction, but this was in a setting with a much higher vaccination uptake of around $90 \%$ [7]. Likewise, in a community-randomized trial with moderate vaccination uptake, significant herd effects among unvaccinated women could only be demonstrated in the sex-neutral vaccination arm, and not in the girls-only vaccination arm [19]. Presumably, the speed at which herd 
effects become apparent is a composite of VE, which is lower against HPV31/33/45 as compared to HPV16/18, and vaccination coverage. Therefore, we suspect herd protection against cross-protective HPV types will also become apparent in unvaccinated women in the Netherlands with prolonged follow-up.

Besides decreasing trends in HPV types, we also observed increasing trends for a few HPV types; for HPV54/56 among all women, and for HPV52 among unvaccinated women. Increasing trends in HPV58/53 were borderline non-significant among (unvaccinated) women. Interestingly, HPV53/54/56 are phylogenetically very distant from the vaccine types and are therefore the least likely to benefit from cross-reactivity of vaccine-induced immune responses [20]. Together, these findings could signify early effects of typereplacement, but an increasing HPV prevalence over time could also be due to unmasking and secular trends irrespective of vaccination, for example due to behavioral changes over time [21]. No significantly increasing trends in HPV prevalence were observed among men, and all increases disappeared in sensitivity analyses. However, detection of typereplacement in our study was complicated by the SHC policy change during our study period, and adjusting for this policy change could have resulted in an overcorrection. Other studies assessing trends in type-specific HPV prevalence have also shown increases in HPV types following vaccination implementation. In meta-analyses, increases were observed in HPV39/52/53/73 [22], and in a community-randomized trial a tendency for increasing prevalence of HPV39/51 was observed among unvaccinated participants [23]. In both studies, results were inconsistent when analyzed by age or birth cohort, and other studies reported no increases in HPV types [24, 25]. Because type-replacement following HPV vaccination probably takes many years to develop if present [12], continued surveillance is needed on a type-specific level. Additionally, eventual replacement in disease burden depends on the oncogenic potential of HPV types becoming more common, emphasizing the need for type-specific surveillance in (pre)cancer screening following vaccination implementation.

The current study has some limitations. First, the prioritization of high-risk individuals eligible for testing at SHCs has changed due to policy changes over the last years. While we corrected for this by including both individual- and population-level confounders in our model, we cannot rule out residual bias. Still, declines in prevalence were already observed without adjustment, both for vaccine-targeted and cross-protective types. Second, vaccination status was self-reported and could be subject to recall bias. If part of the unvaccinated women were truly vaccinated, this would result in an overestimation of the decreasing trends for vaccine types among unvaccinated women. However, previous confirmation analyses based on serology showed good correlations between self-reported vaccination status and observed antibody levels [4]. Therefore, the possible bias by using 
self-reported vaccination status in this setting can be considered minimal. Third, only 1 year of official pre-vaccination data was available, affecting our ability to consider possible background trends or natural fluctuations in HPV prevalence. In sensitivity analyses, we repeated all trend analyses in which study years 2009 and 2011 were pooled and considered to represent the pre-vaccination situation, and these yielded comparable results.

Our current findings indicate that transmission of vaccine-targeted and cross-protective HPV types is decreasing throughout the population. Our study included a high-risk population with a higher HPV prevalence compared to the general Dutch population hampering generalizability [26]. As the population-level impact of HPV vaccination is generally attenuated in a high-prevalence setting, the estimates of herd protection as provided in this study are probably conservative and population effects of girls-only HPV16/18 vaccination in the Netherlands are likely to be stronger in the general population [27]. Our findings also emphasize the importance of monitoring non-vaccine HPV types. Prevalence changes of hrHPV types other than HPV16/18 are relevant to assess the residual risk of (pre-)cancerous lesions and screening needs in vaccinated cohorts. Our results showed declining pooled trends in all hrHPV types and all hr- and IrHPV types together, among both women and heterosexual men. This is reassuring for the overall benefit of HPV16/18 vaccination and demonstrates that the $2 \mathrm{vHPV}$ vaccine generates broad-spectrum protection against HPV infections.

In conclusion, the current study showed substantial population-level impact of girls-only HPV16/18 vaccination in a high-risk study population in the Netherlands, a country with moderate vaccination coverage. Apart from significant declines in vaccine-type HPV infections, we also demonstrated that cross-protection of HPV16/18 vaccination extended to unvaccinated individuals. Our study provides unique documentation of the unfolding of first- and second-order herd effects, and suggests a significant eventual clinical impact of a girls-only HPV vaccination program.

\section{References}

1. de Martel C, et al. Worldwide burden of cancer attributable to HPV by site, country and HPV type. Int J Cancer 2017; 141:664-70.

2. Qendri V, et al. Ten years of HPV vaccination in the Netherlands: current evidence and future challenges in HPV-related disease prevention. Expert Rev Vaccines 2018; 17:1093-104.

3. van Lier EA, et al. [Vaccinatiegraad en jaarverslag Rijksvaccinatieprogramma Nederland 2018]. Bilthoven: RIVM, 2019. Nr: 2019-0015.
4. Woestenberg PJ, et al. Bivalent Vaccine Effectiveness Against Type-Specific HPV Positivity: Evidence for Cross-Protection Against Oncogenic Types Among Dutch STI Clinic Visitors. J Infect Dis 2018; 217:213-22.

5. Donken R, et al. High Effectiveness of the Bivalent Human Papillomavirus (HPV) Vaccine Against Incident and Persistent HPV Infections up to 6 Years After Vaccination in Young Dutch Women. J Infect Dis 2018; 217:1579-89. 
6. Korostil IA, et al. Near elimination of genital warts in Australia predicted with extension of human papillomavirus vaccination to males. Sex Transm Dis 2013; 40:833-5.

7. Kavanagh $\mathrm{K}$, et al. Changes in the prevalence of human papillomavirus following a national bivalent human papillomavirus vaccination programme in Scotland: a 7-year cross-sectional study. Lancet Infect Dis 2017; 17:1293-302.

8. Machalek DA, et al. Very Low Prevalence of Vaccine Human Papillomavirus Types Among 18- to 35-Year Old Australian Women 9 Years Following Implementation of Vaccination. J Infect Dis 2018; 217:1590-600.

9. Drolet $\mathrm{M}$, et al. Population-level impact and herd effects following the introduction of human papillomavirus vaccination programmes: updated systematic review and meta-analysis. Lancet 2019; 394:497-509.

10. Woestenberg PJ, et al. Assessment of herd effects among women and heterosexual men after girls-only HPV16/18 vaccination in the Netherlands: A repeated cross-sectional study. Int J Cancer 2019; 144:2718-27.

11. Malagon $\mathrm{T}$, et al. Human papillomavirus vaccination and the role of herd effects in future cancer control planning: a review. Expert Rev Vaccines 2018; 17:395-409.

12. Man I, et al. Human papillomavirus genotype replacement: still too early to tell? J Infect Dis 2020.

13. Vriend $\mathrm{HJ}$, et al. Type-specific human papillomavirus infections among young heterosexual male and female STI clinic attendees. Sex Transm Dis 2012; 39:72-8.

14. Visser $M$, et al. Sexually transmitted infections in the Netherlands in 2016. Bitlhoven: RIVM, 2017. Nr: 2017-0003; DOI: 10.21945/RIVM-2017-0003.

15. Vriend $\mathrm{HJ}$, et al. Patterns of human papillomavirus DNA and antibody positivity in young males and females, suggesting a site-specific natural course of infection. PLoS One 2013; 8:e60696.

16. Woestenberg PJ, et al. HPV infections among young MSM visiting sexual health centers in the Netherlands: Opportunities for targeted HPV vaccination. Vaccine 2020; 38:3321-9.

17. Oliver SE, et al. Prevalence of Human Papillomavirus Among Females After Vaccine
Introduction-National Health and Nutrition Examination Survey, United States, 2003-2014. J Infect Dis 2017; 216:594-603.

18. Malagon T, et al. Cross-protective efficacy of two human papillomavirus vaccines: a systematic review and meta-analysis. Lancet Infect Dis 2012; 12:781-9. 19. Lehtinen $M$, et al. Gender-neutral vaccination provides improved control of human papillomavirus types 18/31/33/35 through herd immunity: Results of a community randomized trial (III). Int J Cancer 2018; 143:2299-310.

20. Bogaards JA, et al. Bivalent Human Papillomavirus (HPV) Vaccine Effectiveness Correlates With Phylogenetic Distance From HPV Vaccine Types 16 and 18. J Infect Dis 2019; 220:11416.

21. Tota JE, et al. Epidemiologic approaches to evaluating the potential for human papillomavirus type replacement postvaccination. Am J Epidemiol 2013; 178:625-34.

22. Mesher D, et al. Population-Level Effects of Human Papillomavirus Vaccination Programs on Infections with Nonvaccine Genotypes. Emerg Infect Dis 2016; 22:1732-40.

23. Gray P, et al. Evaluation of HPV type-replacement in unvaccinated and vaccinated adolescent femalesPost-hoc analysis of a community-randomized clinical trial (II). Int J Cancer 2018; 142:2491-500.

24. Mesher D, et al. The Impact of the National HPV Vaccination Program in England Using the Bivalent HPV Vaccine: Surveillance of Type-Specific HPV in Young Females, 2010-2016. J Infect Dis 2018; 218:911-21.

25. Feiring B, et al. Substantial Decline in Prevalence of Vaccine-Type and Nonvaccine-Type Human Papillomavirus (HPV) in Vaccinated and Unvaccinated Girls 5 Years After Implementing HPV Vaccine in Norway. J Infect Dis 2018; 218:1900-10.

26. Mollers $M$, et al. Population- and type-specific clustering of multiple HPV types across diverse risk populations in the Netherlands. Am J Epidemiol 2014; 179:1236-46.

27. Baussano I, et al. Impacts of human papillomavirus vaccination for different populations: A modeling study. Int J Cancer 2018; 143:1086-92. 


\section{Supplementary information to Chapter 6}

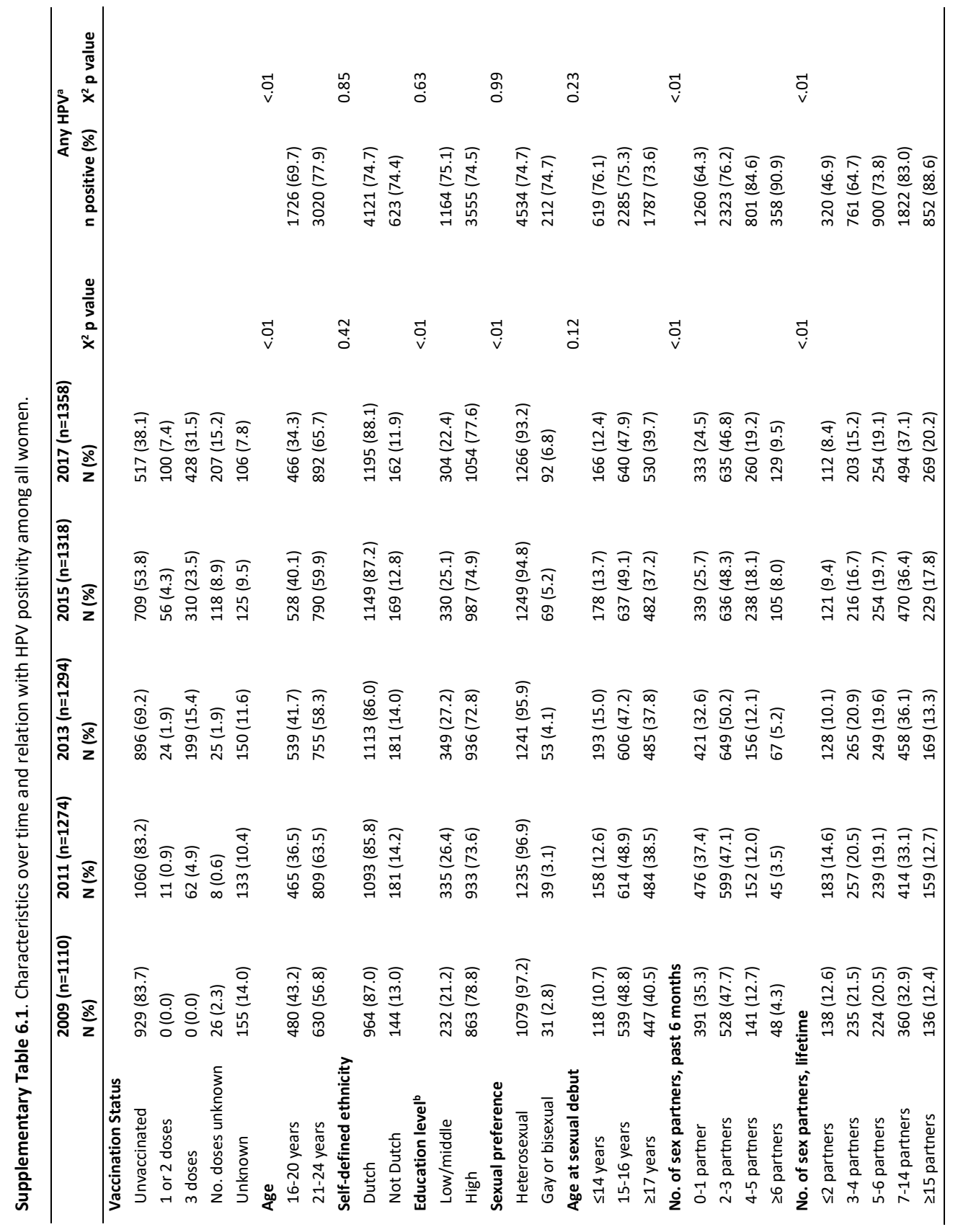




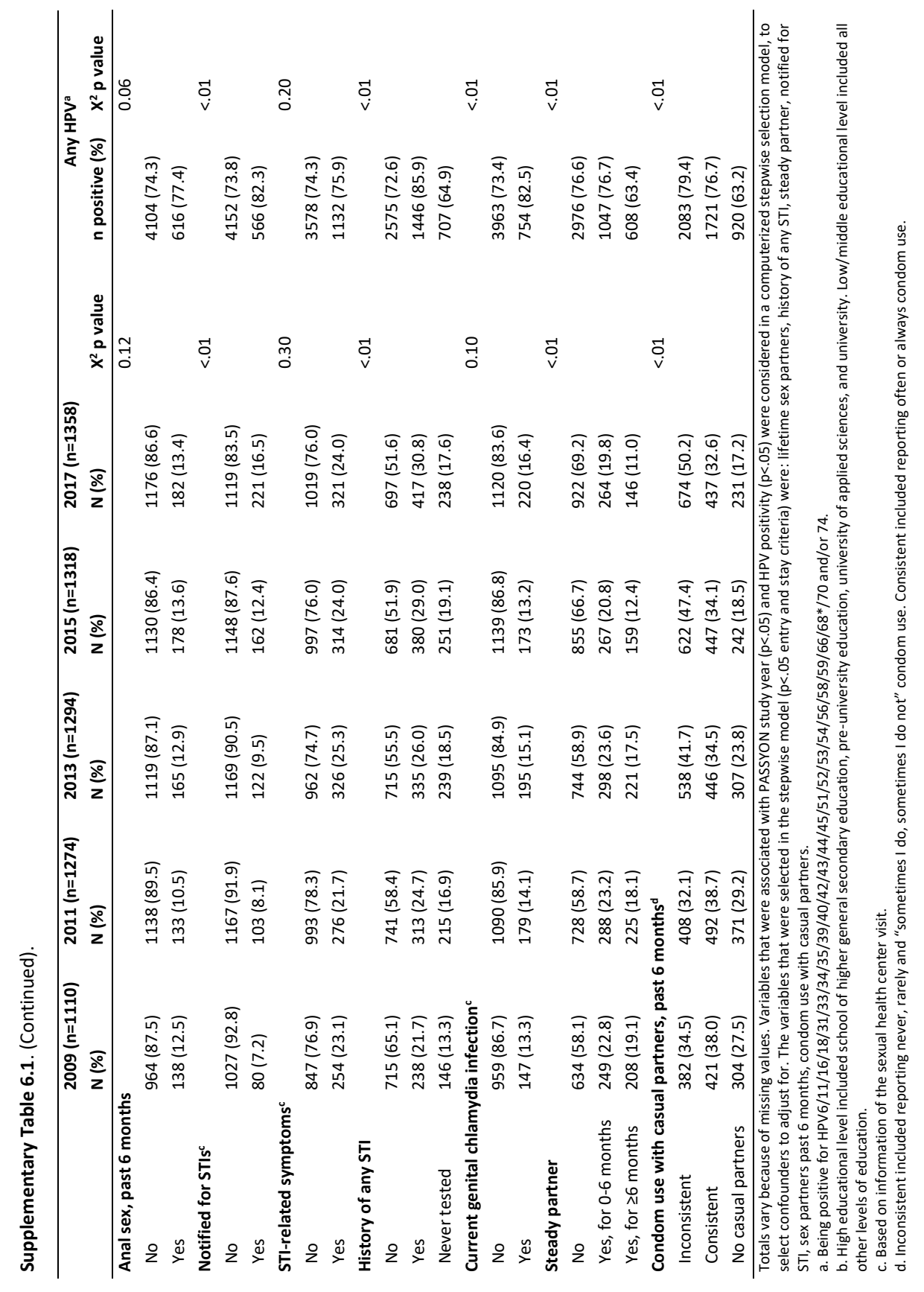




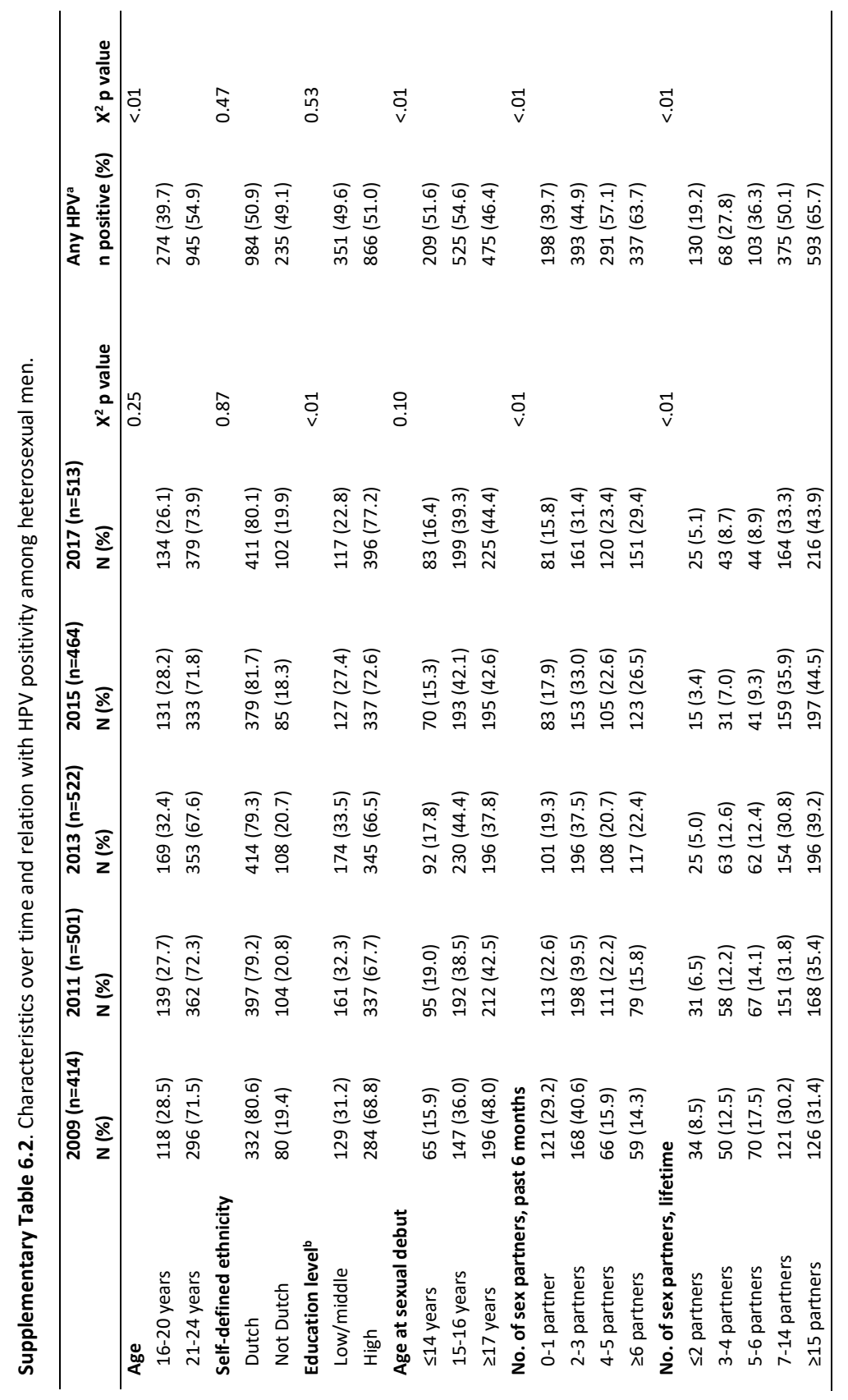




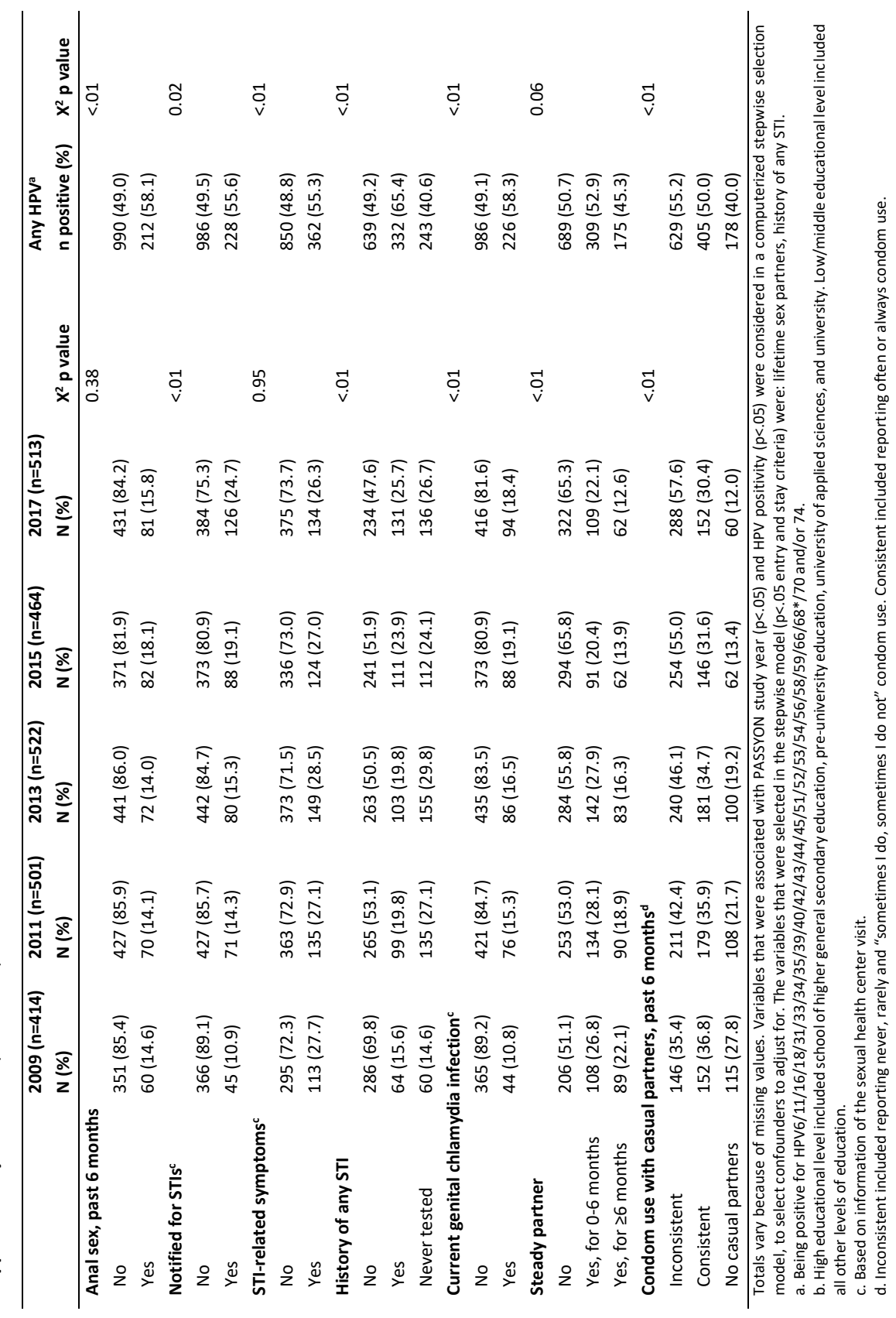




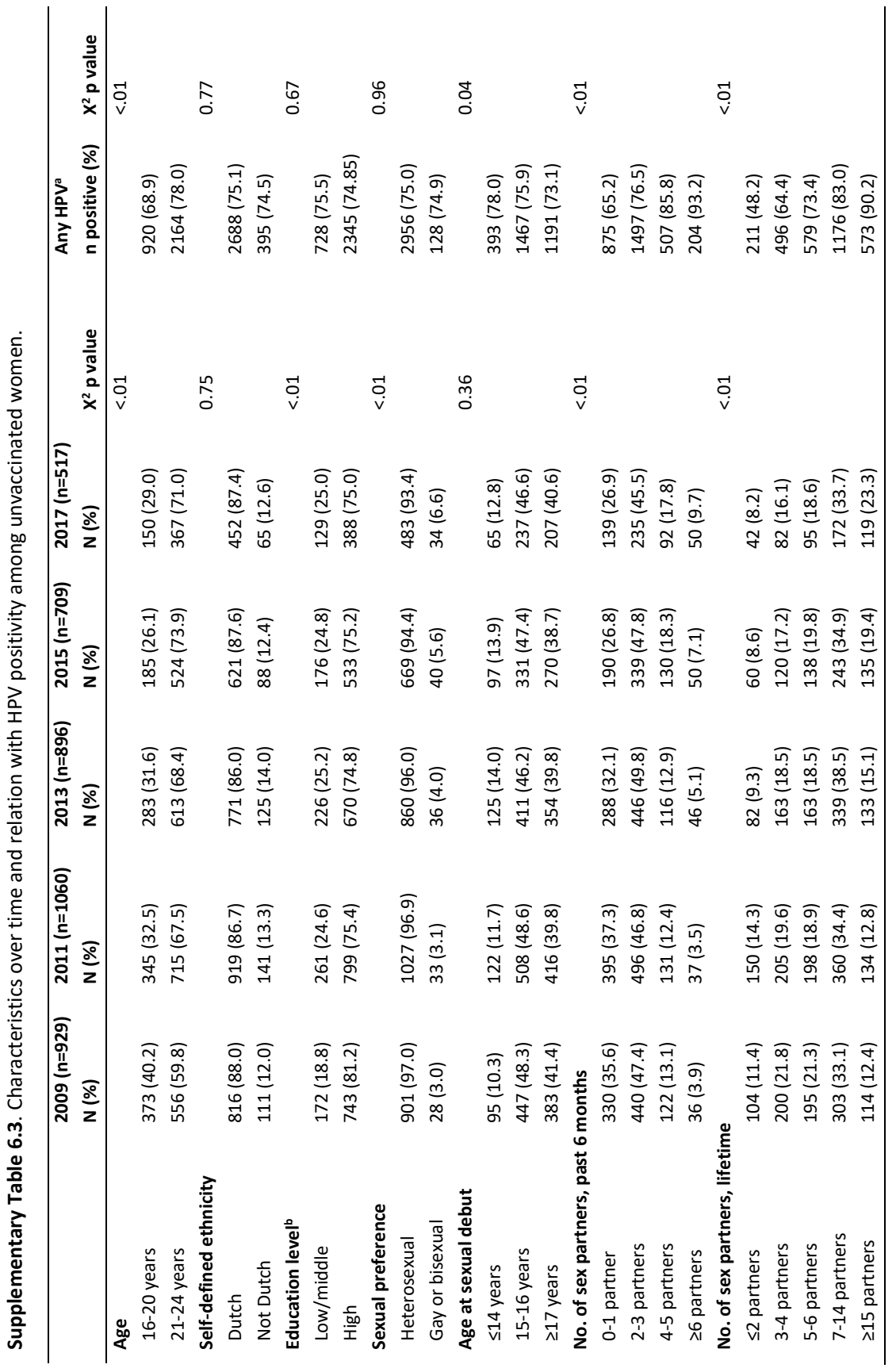




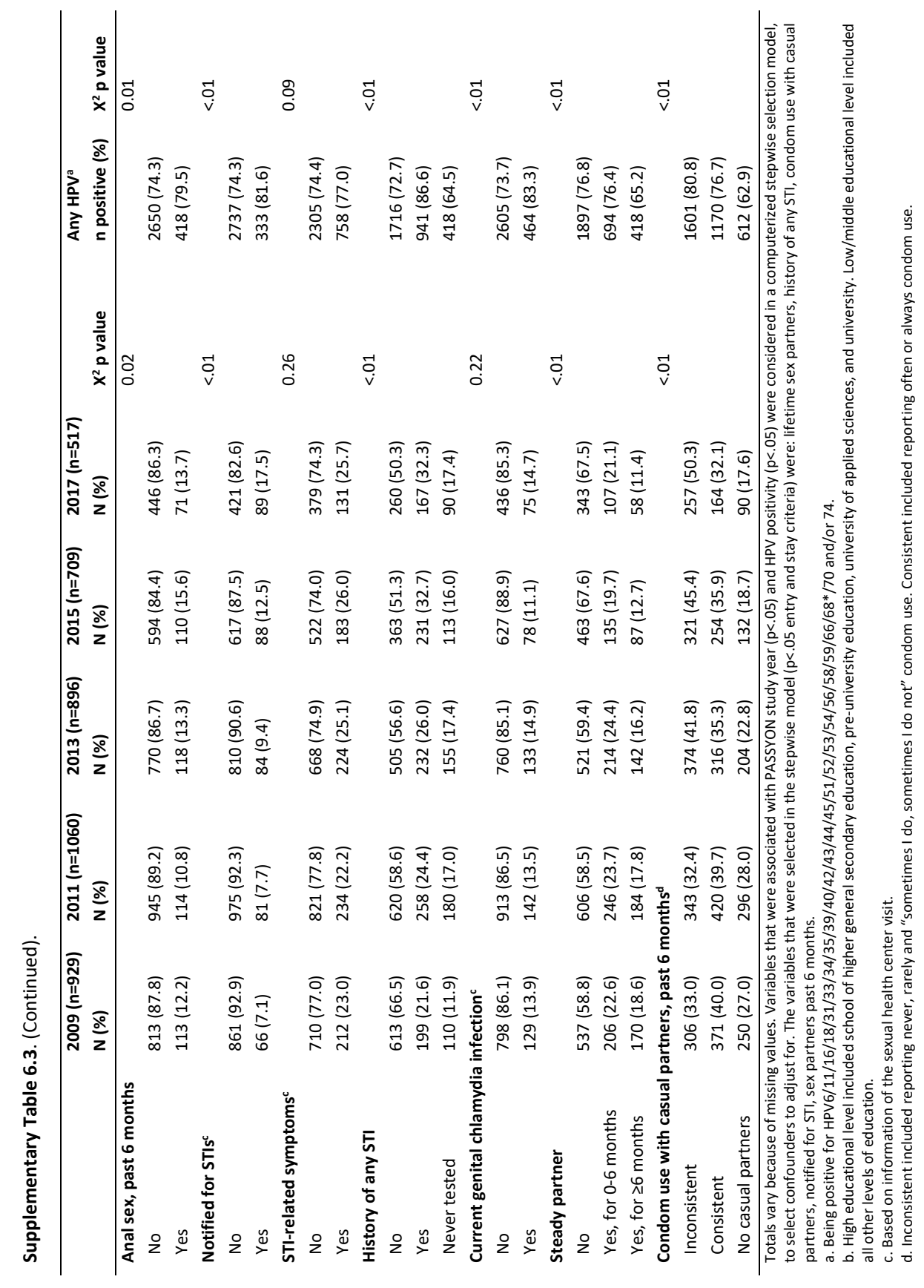




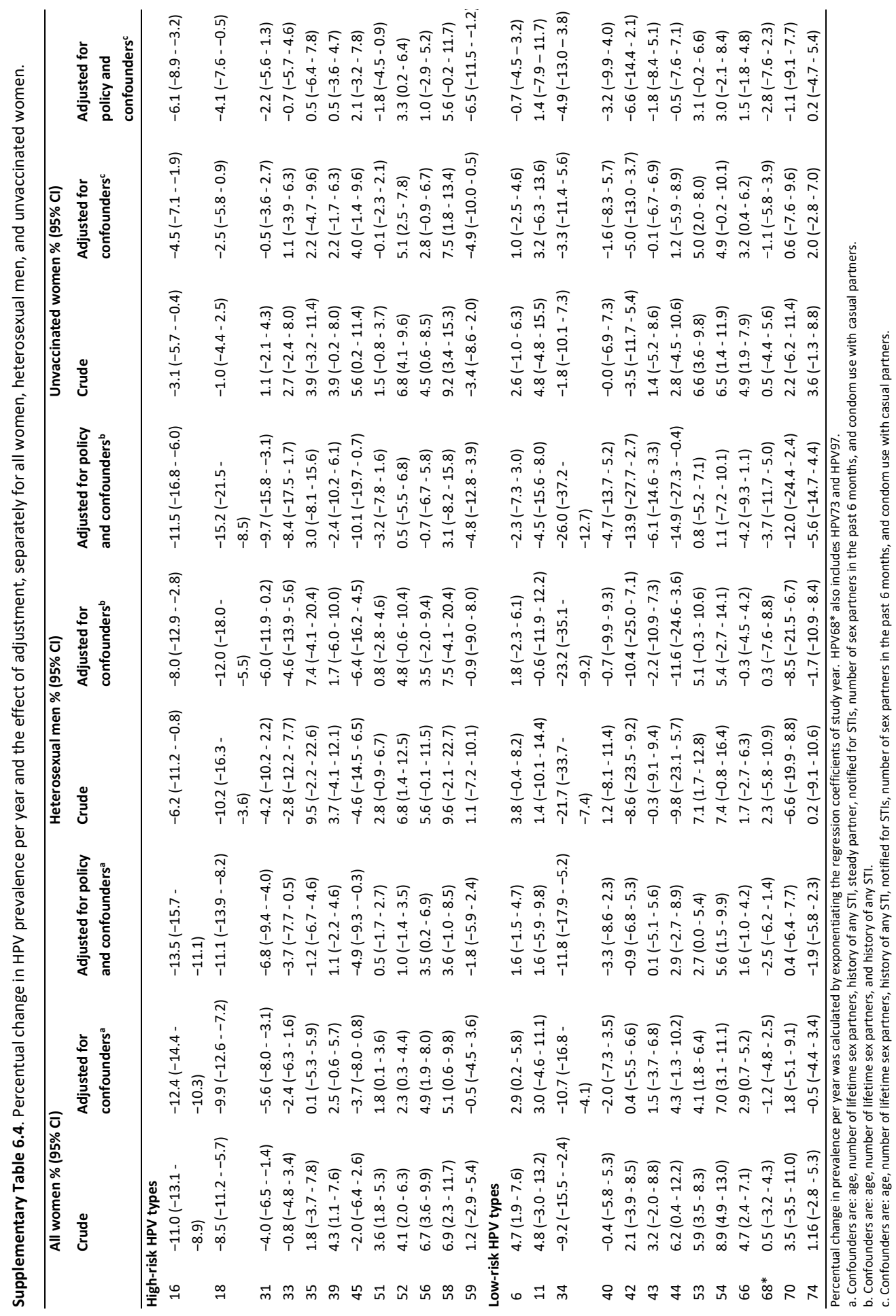


Supplementary Table 6.5. Percentual change in HPV prevalence per year of sensitivity analyses pooling data of PASSYON study rounds 2009 and 2011.

\begin{tabular}{|c|c|c|c|}
\hline & $\begin{array}{l}\text { All women } \\
\%(95 \% \mathrm{Cl})\end{array}$ & $\begin{array}{l}\text { Heterosexual men } \\
\%(95 \% \mathrm{Cl})\end{array}$ & $\begin{array}{l}\text { Unvaccinated women } \\
\%(95 \% \mathrm{Cl})\end{array}$ \\
\hline \multicolumn{4}{|c|}{ High-risk HPV types } \\
\hline 16 & $-18.75(-21.74$ to -15.67$)$ & $-10.68(-17.47$ to -3.34$)$ & $-9.29(-13.11$ to -5.30$)$ \\
\hline 18 & $-17.05(-20.71$ to -13.21$)$ & $-16.60(-25.04$ to -7.21$)$ & $-8.51(-13.16-3.60)$ \\
\hline 31 & $-9.67(-13.01$ to -6.19$)$ & $-7.86(-15.58-0.55)$ & $-3.78(-8.16-0.81)$ \\
\hline 33 & $-6.64(-11.47$ to -1.54$)$ & $-7.36(-18.66-5.49)$ & $-3.14(-9.40-3.54)$ \\
\hline 35 & $-2.66(-9.17-4.32)$ & $5.73(-7.03-20.25)$ & $-0.79(-9.20-8.39)$ \\
\hline 39 & $-1.00(-5.02-3.20)$ & $0.03(-9.48-10.54)$ & $-1.38(-6.52-4.04)$ \\
\hline 45 & $-10.26(-15.73$ to -4.44$)$ & $-11.50(-23.65-2.58)$ & $-1.51(-8.23-5.70)$ \\
\hline 51 & $-1.34(-4.15-1.55)$ & $-1.40(-7.43-5.03)$ & $-3.82(-7.42$ to -0.09$)$ \\
\hline 52 & $-0.82(-3.89-2.36)$ & $3.37(-4.10-11.43)$ & $2.48(-1.49-6.61)$ \\
\hline 56 & $2.11(-1.84-6.23)$ & $0.10(-7.76-8.62)$ & $-0.41(-5.42-4.86)$ \\
\hline 58 & $2.27(-3.18-8.03)$ & $5.92(-7.52-21.32)$ & $4.93(-2.04-12.38)$ \\
\hline 59 & $-5.19(-10.17-0.07)$ & $-4.98(-14.93-6.13)$ & $-12.23(-18.97$ to -4.94$)$ \\
\hline \multicolumn{4}{|c|}{ Low-risk HPV types } \\
\hline 6 & $0.20(-3.59-4.13)$ & $0.62(-5.79-7.48)$ & $-2.15(-6.97-2.93)$ \\
\hline 11 & $0.95(-7.80-10.53)$ & $-0.46(-13.49-14.55)$ & $1.53(-9.48-13.87)$ \\
\hline 34 & $-15.37(-22.55$ to -7.53$)$ & $-30.00(-45.01$ to -10.89$)$ & $-7.58(-17.14-3.08)$ \\
\hline 40 & $-5.73(-12.01-0.99)$ & $-6.07(-17.59-7.07)$ & $-4.86(-12.98-4.02)$ \\
\hline 42 & $-1.89(-8.67-5.39)$ & $-16.01(-33.48-6.05)$ & $-8.24(-17.58-2.16)$ \\
\hline 43 & $-2.01(-8.21-4.60)$ & $-6.69(-17.53-5.57)$ & $-3.63(-11.64-5.11)$ \\
\hline 44 & $2.54(-3.88-9.40)$ & $-11.17(-26.30-7.04)$ & $-0.67(-9.09-8.53)$ \\
\hline 53 & $1.19(-2.17-4.66)$ & $4.44(-3.21-12.67)$ & $2.37(-1.95-6.87)$ \\
\hline 54 & $4.47(-0.39-9.56)$ & $4.11(-6.00-15.33)$ & $2.04(-4.25-8.75)$ \\
\hline 66 & $-0.63(-3.87-2.73)$ & $-2.72(-9.30-4.33)$ & $-0.83(-4.99-3.53)$ \\
\hline $68^{*}$ & $-4.97(-9.53$ to -0.18$)$ & $-0.18(-9.60-10.21)$ & $-4.88(-10.93-1.59)$ \\
\hline 70 & $-0.86(-8.60-7.54)$ & $-10.72(-25.98-7.69)$ & $-2.33(-11.84-8.20)$ \\
\hline 74 & $-5.25(-10.03$ to -0.21$)$ & $-3.78(-14.63-8.45)$ & $-2.30(-8.41-4.21)$ \\
\hline \multicolumn{4}{|c|}{ Pooled outcome measures } \\
\hline $2 v$ types $^{d}$ & $-18.13(-20.76$ to -15.41$)$ & $-13.03(-19.10$ to -6.51$)$ & $-9.01(-12.42$ to -5.46$)$ \\
\hline $9 v$ hr types $^{\mathrm{e}}$ & $-8.92(-11.23$ to -6.56$)$ & $-6.27(-11.66$ to -0.55$)$ & $-3.41(-6.44$ to -0.28$)$ \\
\hline All hr types ${ }^{f}$ & $-5.46(-7.65$ to -3.21$)$ & $-3.56(-8.63-1.80)$ & $-3.37(-6.21$ to -0.44$)$ \\
\hline All types ${ }^{\mathrm{g}}$ & $-3.61(-5.79$ to -1.39$)$ & $-1.79(-6.77-3.46)$ & $-2.52(-5.31-0.35)$ \\
\hline \multicolumn{4}{|c|}{$\begin{array}{l}\text { Percentual change in prevalence per year was calculated by exponentiating the adjusted regression coefficients of study year. } \\
\text { Pooled estimates were obtained as a weighted average of the type-specific trends in the GEE Poisson models. HPV68* also } \\
\text { includes HPV73 and HPV97. } \\
\text { a. Adjusted for age, policy change at the SHC, number of lifetime sex partners, history of any STI, steady partner, notified for } \\
\text { STIs, number of sex partners in the past } 6 \text { months, and condom use with casual partners. } \\
\text { b. Adjusted for age, policy change at the SHC, number of lifetime sex partners, and history of any STI. } \\
\text { c. Adjusted for age, policy change at the SHC, number of lifetime sex partners, history of any STI, notified for STls, number of } \\
\text { sex partners in the past } 6 \text { months, and condom use with casual partners. } \\
\text { d. Including HPV types } 16,18 \text {. } \\
\text { e. Including HPV types } 16,18,31,33,45,52,58 \text {. } \\
\text { f. Including HPV types } 16,18,31,33,45,52,58,35,39,51,56,59 . \\
\text { g. Including HPV types } 16,18,31,33,45,52,58,35,39,51,56,59,6,11,34,40,42,43,44,53,54,66,70,74,68 / 73 / 97 \text {. }\end{array}$} \\
\hline
\end{tabular}





\section{Chapter 7}

\section{HPV infections among young MSM visiting sexual health centers in the Netherlands: Opportunities for targeted HPV vaccination}

Petra J. Woestenberg

Birgit H. B. van Benthem Johannes A. Bogaards

Audrey J. King

Fiona R. van der Klis

Hella Pasmans

Suzan Leussink

Marianne A. B. van der Sande

Christian J. P. A. Hoebe

The contents of this chapter have been published in Vaccine

Vaccine. 2020; 38:3321-9 


\section{Abstract}

Introduction: In 2009, girls-only HPV16/18 vaccination was introduced in the Netherlands which has achieved $46 \%-61 \%$ uptake. Heterosexual men have benefitted from herd protection, but it is unknown whether men who have sex with men (MSM) also benefit from herd effects of the girls-only HPV16/18 vaccination program. Because MSM bear a high HPV-related disease burden, countries might consider targeted vaccination for MSM. To study possible herd effects and prior HPV exposure at a potential moment of vaccination, we assessed trends in the HPV prevalence and proportions (sero)negative for the various vaccine types among young MSM visiting sexual health centers (SHCs).

Methods: We used data from MSM included in PASSYON study years 2009-2017. In this biennial cross-sectional study among visitors of SHCs aged 16-24 years, MSM provided a penile and anal swab for HPV DNA testing (including vaccine types HPV6/11/16/18/31/33/ 45/52/58) and blood for HPV antibody testing (HPV16/18/31/33/45/52/58).

Results: In total 575 MSM were included, with a median of 22 years of age and 15 lifetime sex partners and 3.5\% HIV positive. Trends in penile or anal HPV prevalence during 20092017 were statistically non-significant for all vaccine types. Of the 455 MSM with a penile and anal swab, 360 (79\%), 283 (62\%), and 242 (53\%) were HPV DNA negative at both anatomical sites for HPV16/18, HPV6/11/16/18, and HPV6/11/16/18/31/33/45/52/58 respectively. Among MSM who were HPV16/18 and HPV16/18/31/33/45/52/58 DNA negative and were tested for serology ( $n=335$ and 279 respectively), $82 \%$ and $71 \%$ were also seronegative for the respective types.

Discussion: There were no significant declines in the HPV prevalence among MSM up to 8 years after introduction of girls-only HPV16/18 vaccination, indicating that MSM are unlikely to benefit largely from herd effects from girls-only vaccination. Most MSM were vaccine-type DNA negative and seronegative, suggesting that vaccination of young MSM visiting SHCs could still be beneficial. 


\section{Introduction}

Sexually transmitted human papillomavirus (HPV) can cause anogenital warts (AGWs) and various cancers in both men and women: cervical, vaginal, vulvar, anal, and oropharyngeal cancer in women; and penile, anal, and oropharyngeal cancer in men [1]. Many different HPV types have been identified, which are classified into high-risk HPV (hrHPV) or low-risk HPV (IrHPV) based on their oncogenic potential. Currently, 3 prophylactic vaccines against HPV are on the European market and all are licensed for both males and females [2-4]; a bivalent HPV (2vHPV), quadrivalent HPV (4vHPV), and nonavalent HPV (9vHPV) vaccine. All vaccines target hrHPV types $16 / 18$. The $4 \mathrm{VHPV}$ and $9 \mathrm{vHPV}$ vaccines also target IrHPV types $6 / 11$, the most important types causing AGWs [5]. The 9vHPV vaccine targets 5 additional hrHPV types: HPV31/33/45/52/58. As of May 2018, nearly half of the countries worldwide have implemented HPV vaccination in their national immunization program (NIP) [6]. Studies of high-income countries have shown declines in the HPV infection prevalence and the burden of AGWs and pre-malignant disease within a decade after HPV vaccination implementation [7].

Although sex-neutral HPV vaccination has been implemented in a diverse array of countries (e.g. Argentina, Australia, Austria, Brazil, Canada, Croatia, Israel, Panama) to prevent HPV-related cancers in both men and women, many countries still offer vaccination to girls only [8]. Also in the Netherlands, HPV vaccination is still a girls-only program as of 2020. 2vHPV vaccination was introduced in 2009 with the main aim to prevent cervical cancer. It started with a catch-up campaign for 12- to 16-year-old girls and in $2010 \mathrm{HPV}$ vaccination was implemented in the Dutch NIP for girls in the calendar year they turn 13 years old. The vaccination uptake has ranged between $46 \%$ and $61 \%$ in vaccine-eligible cohorts [9].

Among heterosexual men, declines in the HPV vaccine-type prevalence have been observed after introduction of girls-only HPV vaccination, indicating that heterosexual men benefit from herd protection $[10,11]$. It is unknown whether men who have sex with men (MSM) also experience decreases in the HPV16/18 prevalence as observed among heterosexual men. In Australia, AGWs (mostly caused by HPV6/11) nearly disappeared in young Australian heterosexual men within 7 years of girls-only 4vHPV vaccination, whereas only a small decline in AGWs was observed among MSM [12]. Accordingly, herd protection for hrHPV types among MSM is expected to be less than for heterosexual men, even though MSM are at much higher risk of HPV-related diseases than heterosexual men, especially for anal cancer. In meta-analyses published in 2012, the anal cancer incidence was estimated at 5.1 per 100,000 among HIV negative MSM and at 45.9 per 100,000 among HIV positive MSM [13]. This is about 17-30 times more frequent compared to heterosexual men [14, 15], 
highlighting the importance of extending the protection afforded by prophylactic HPV vaccination to MSM.

Additional to (pre)adolescent sex-neutral vaccination, countries might consider targeted vaccination for MSM. When combined with sex-neutral vaccination in (pre)adolescence, additional vaccination of MSM, even when previously exposed to HPV, is predicted to accelerate penile and anal cancer prevention, compensate for low-uptake among (pre)adolescents and protect previously unvaccinated $\operatorname{MSM}[16,17]$. A randomized controlled trial (RCT) carried out in MSM aged up to 26 years with 1-5 lifetime sex partners, showed that vaccination is effective in preventing genital and anal lesions, especially in those DNA negative and seronegative for the HPV vaccine type under study and at the anatomical site under study $[18,19]$. Because it is difficult to target MSM from the general population and before sexual debut, an option would be to offer vaccination to MSM visiting sexual health centers (SHCs), comparable to targeted hepatitis B vaccination [20]. This is already being implemented in for example the United Kingdom after a successful pilot program with nearly $50 \%$ uptake [21]. However, the effectiveness of HPV vaccination targeting sexually active MSM visiting SHCs might be hampered by prior exposure to HPV vaccine types.

Here, we assessed the scope of targeted HPV vaccination for MSM attending SHCs. First, we assessed trends in the penile and anal HPV prevalence among MSM visiting SHCs in the Netherlands from pre-vaccination up to 8 years post-vaccination, to study possible herd effects from girls-only vaccination. Second, we assessed the proportions HPV DNA negative at the penile and anal site and seronegative for the various vaccine-targeted types, to study prior exposure and the occurrence of prevalent infections at a potential moment of targeted vaccination, i.e. directed at MSM upon SHC visits.

\section{Methods}

\section{Study design and population}

We used data from the PASSYON (PApillomavirus Surveillance among STI clinic YOungsters in the Netherlands) study, a biennial cross-sectional survey among 16- to 24-year-old visitors to SHCs in the Netherlands that started in 2009 when girls-only 2vHPV vaccination was implemented [22]. In the current analysis we used data from MSM included in the PASSYON study. MSM were classified as men who indicated to be homosexual or bisexual in the questionnaire. In addition to routine sexually transmitted infection (STI) testing, MSM were asked to provide a self-collected penile and anal swab for HPV testing. For the penile swab, men were instructed to firmly move the swab up and down the entire penile shaft, the glans, the coronal sulcus, and under the foreskin. For the anal swab, men were 
instructed to insert the swab about $3 \mathrm{~cm}$ into the anus and circle it around. From participants who provided blood for routine syphilis and HIV testing, serum was collected to assess their HPV serology-status. Because MSM are at higher risk for syphilis and HIV, testing is usually indicated. The PASSYON study was repeated in 2011, 2013, 2015, and 2017 using the same study protocol during which the proportion of women who had been offered HPV vaccination increased to almost $90 \%$ (of whom almost $60 \%$ reported to be HPV vaccinated with $\geq 1$ dose). Participants could be included in multiple study rounds, but the probability of repeat consultations is low as we sampled for only 2 month in the same period (i.e. February-March) every other year. The Medical Ethical Committee of the University of Utrecht, the Netherlands, approved this study (protocol number 08/397). Data was obtained using a unique code per person and all participants gave informed consent.

\section{Laboratory methods}

Swabs were tested using the SPF10-DEIA-LiPA25 assay (DDL Diagnostics Laboratory, the Netherlands) as published in detail previously [22]. This sensitive broad-spectrum polymerase chain reaction (PCR) is able to detect DNA of 25 HPV types, including the vaccine-targeted HPV types 6/11/16/18/31/33/45/52/58 and the non-vaccine hrHPV types 35/39/51/56/59.

HPV serum IgG antibodies were assessed using a virus-like particle (VLP) based multiplex immunoassay against the vaccine-targeted hrHPV types $16 / 18 / 31 / 33 / 45 / 52 / 58$ as published in detail previously [23, 24]. GSK (GlaxoSmithKline, Rixensart, Belgium) and MSD (Merck\&Co, Kenilworth, NJ, USA) produced the VLPs that were used in the study. Serum samples were considered antibody seropositive at the following previously determined cutoffs: 9, 13, 27, 11, 19, 14, and 31 Luminex Units (LU)/mL for HPV types 16, 18, 31, 33, 45, 52 , and 58, respectively [24].

\section{Statistical analyses}

We explored the association between characteristics of the MSM and hrHPV DNA positivity (being positive for HPV types 16/18/31/33/35/39/45/51/52/56/58 or 59) using $\chi^{2}$ tests, for penile and anal HPV separately. To study trends in the vaccine types over time, we calculated the penile and anal HPV DNA prevalence for each PASSYON study year and performed crude Cochran-Armitage Trend Tests. Changes in the characteristics of the study population by study year were explored using $\chi^{2}$ tests.

Because RCTs showed that vaccine efficacy among women was substantial even if a woman was seropositive when vaccinated (>66\% against persistent infection with the vaccine types) $[25,26]$, we first calculated the proportion DNA negative for the vaccinetargeted HPV types in the penile and anal swab, irrespective of serostatus. We did this among MSM with both swabs available, for the vaccine-targeted HPV types separately as 
well as combined for the types included in the currently licensed vaccines (HPV16/18, HPV6/11/16/18, and HPV6/11/16/18/31/33/45/52/58).

Next, we calculated the proportion DNA negative (both swabs) and seronegative. This was done for the vaccine-targeted hrHPV types only, because serum antibodies against HPV6/11 were not determined, again for each type separately as well as combined (HPV16/18 and HPV16/18/31/33/45/52/58). MSM were considered negative if they were DNA negative for all types in both swabs and seronegative for all types.

Last, to explore the value of seropositivity as a marker of prior exposure to HPV, we studied the HPV antibody concentration by age and number of lifetime sex partners (categorized into 5 categories based on percentiles). The associations between log transformed antibody concentration and age/lifetime sex partners were studied using linear regression. All analyses were performed using SAS version 9.4 (SAS Institute Inc., Cary, NC) with a significance level of $p<.05$.

\section{Results}

\section{Study population}

There were 587 MSM in the PASSYON study of which 575 (98\%) provided a penile and/or anal swab and were included in the current analyses; 71 in round 2009, 110 in round 2011, 136 in round 2013, 130 in round 2015, and 128 in round 2017. In total, 455 (78\%) provided a penile and anal swab, 112 provided only a penile swab, and 8 provided only an anal swab. We had serum available of 531 MSM (92\%) and 421 (73\%) men provided both swabs and serum.

Characteristics of the study population and the association with hrHPV DNA positivity are presented in Table 7.1. The median age of the MSM was 22 years (range 16-24) and the median reported number of lifetime sex partners was 15 (interquartile range: 6-30). Of all MSM, 3.5\% were HIV positive. Overall, $20.3 \%$ and $36.7 \%$ of the MSM were positive for hrHPV at the penile and anal site respectively. In general, higher sexual risk behavior (like a higher number of lifetime sex partners and a history of STIS) was associated with hrHPV positivity. Receptive anal sex in the past 6 months was associated with anal hrHPV and insertive anal sex in the past 6 months with penile hrHPV. 
Table 7.1. Characteristics of the MSM over all PASSYON study years and the relation with hrHPV DNA positivity.

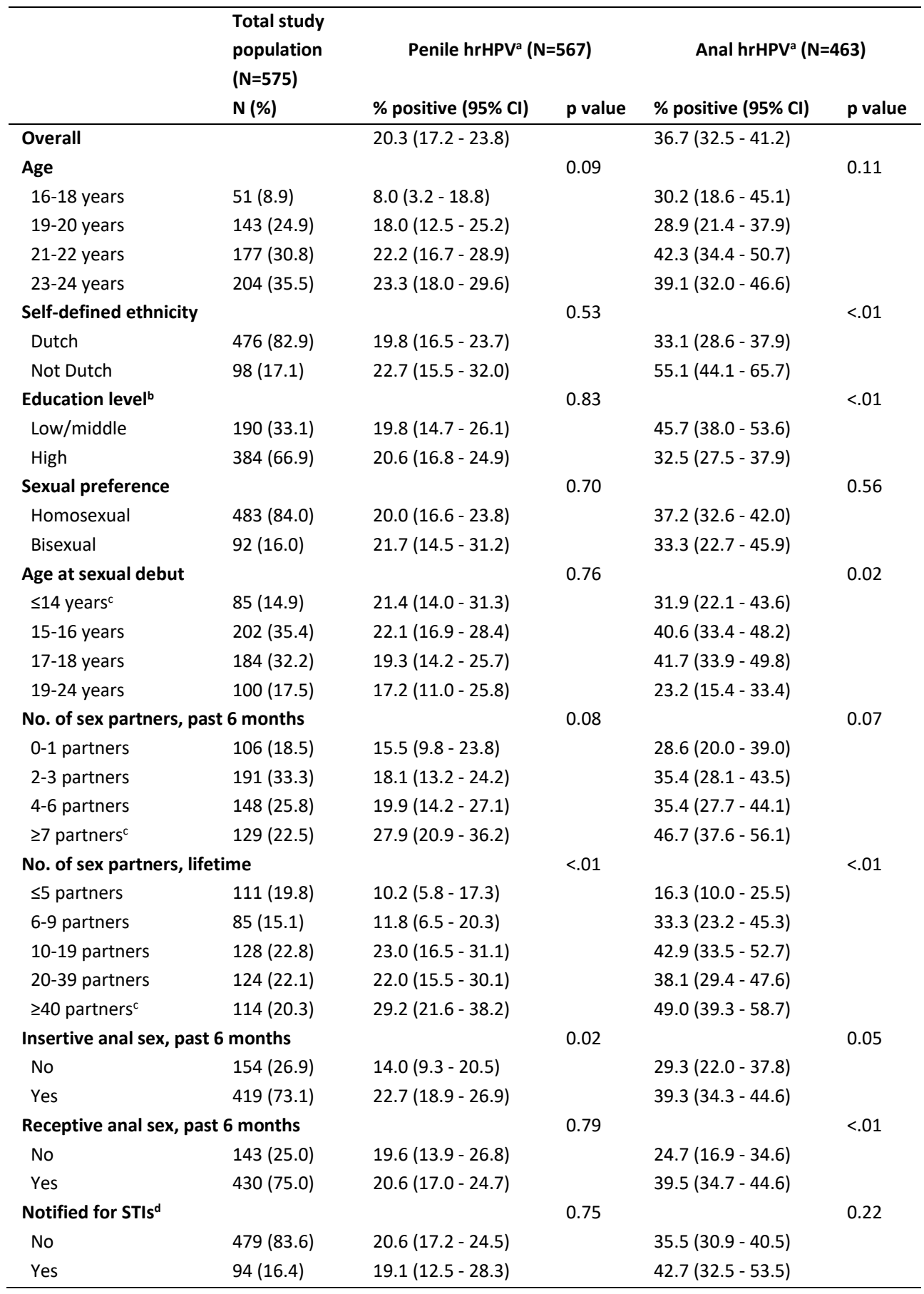


Table 7.1. (Continued).

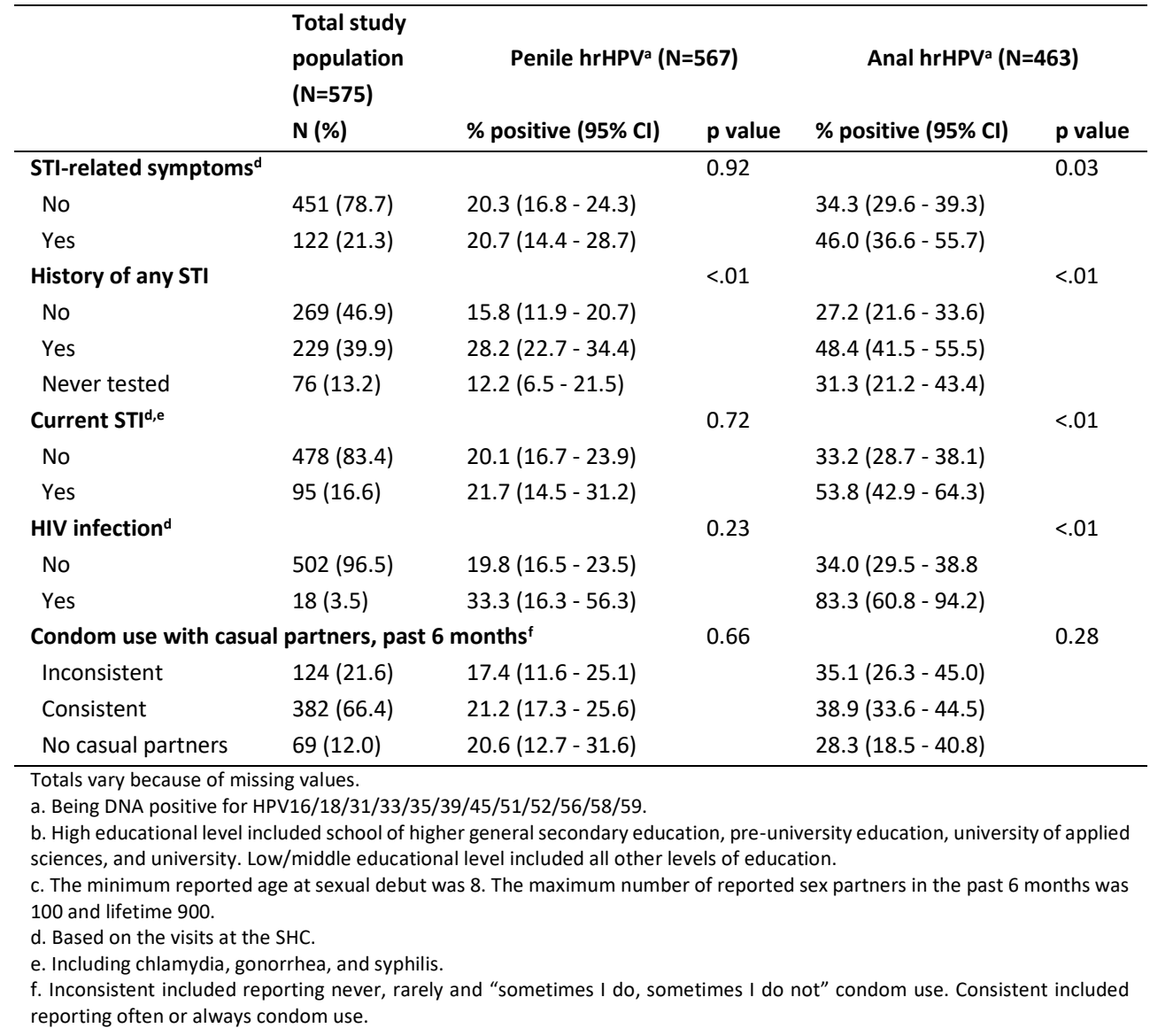

\section{Prevalence of vaccine-targeted HPV types over time}

No statistically significant declining trends were observed in the penile (Figure 7.1A) or anal (Figure 7.1B) HPV DNA prevalence among MSM for any of the vaccine-targeted types up to 8 years after the introduction of girls-only $2 \mathrm{VHPV}$ vaccination. Also for the pooled outcome HPV16/18, no statistically significant declining trend was observed ( $p_{\text {trend }}=0.75$ for penile and $p_{\text {trend }}=0.50$ for anal HPV). The prevalence of AGWs decreased from $7.1 \%$ in 2009 to $0.8 \%$ in 2017 ( $p_{\text {trend }}=0.03$ ) (Figure 7.1C). Changes over time in the characteristics of the MSM included in the PASSYON study are presented in Supplementary Table 7.1. Only sexual preference and a history of STIs were associated with PASSYON study year $(p<.05)$. The proportion reporting no history of STIs was 57\% in 2009 and $41 \%$ in 2017. 
A) Penile HPV

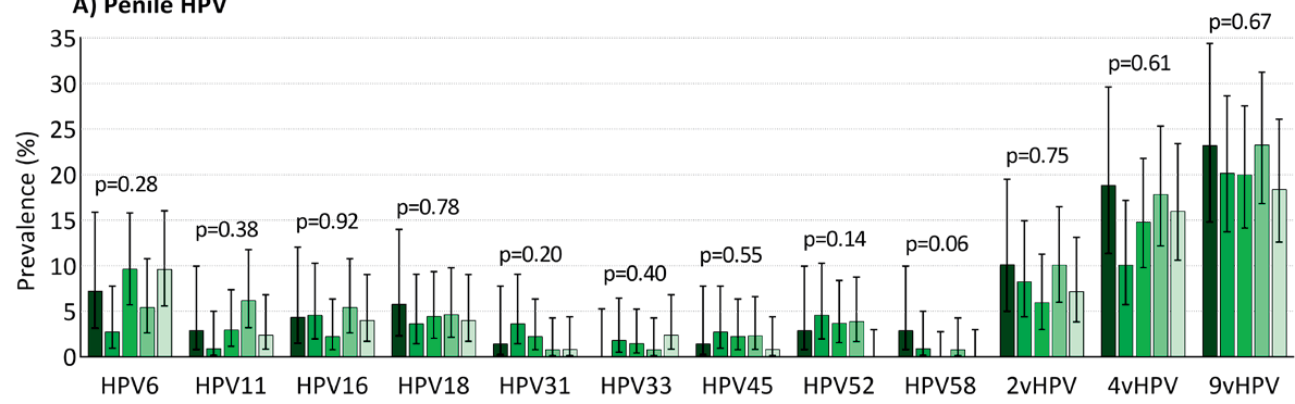

B) Anal HPV

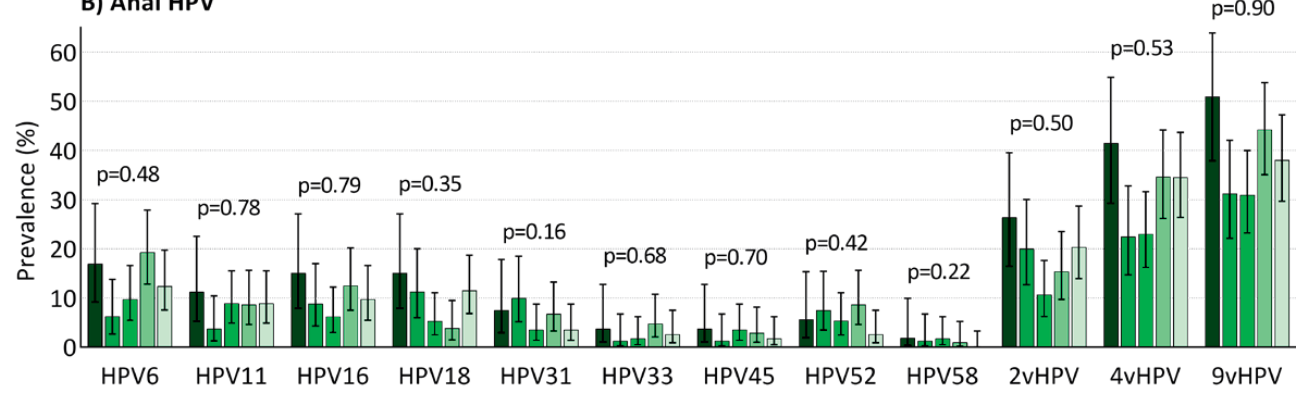

C) Anogenital warts

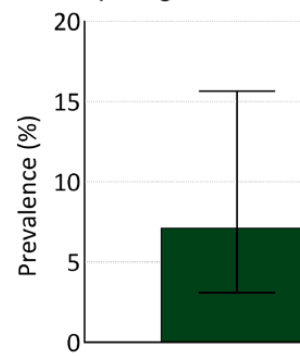

2009 
was positive at both sites. For HPV18, $89 \%$ was negative, $6.8 \%$ was positive only at the anal site, $2.0 \%$ was positive only at the penile site, and $1.8 \%$ was positive at both sites. In total, 79\%, 62\%, and 53\% were HPV DNA negative at both anatomic sites for HPV16/18, HPV6/11/16/18, and HPV6/11/16/18/31/33/45/52/58, respectively (Figure 7.2B). Of the MSM infected with at least one of the 9 vaccine-targeted types at either anatomical site $(n=213)$, the majority was infected with 1 type $(n=136,64 \%)$. Nobody was positive for all vaccine-targeted types; the maximum number of types present at either anatomic site was $6(n=1)$. Nobody was positive for both HPV16 and HPV18 at both anatomic sites.

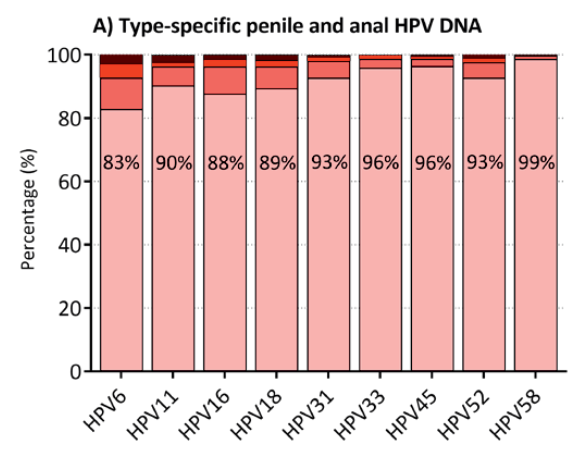

B) Combined penile and anal HPV DNA ${ }^{1}$

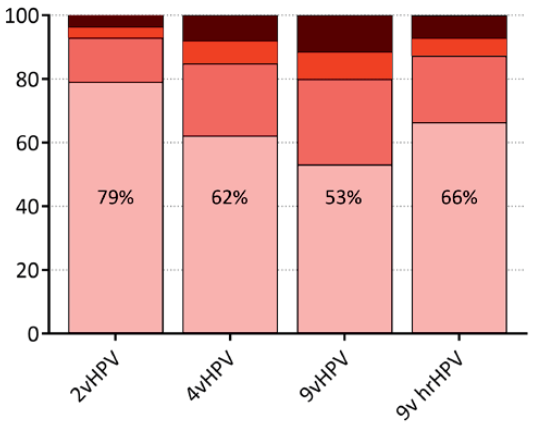

Penile + anal +

Penile + anal -

Penile-anal +

Penile-anal-

C) Type-specific HPV DNA and serology ${ }^{2}$

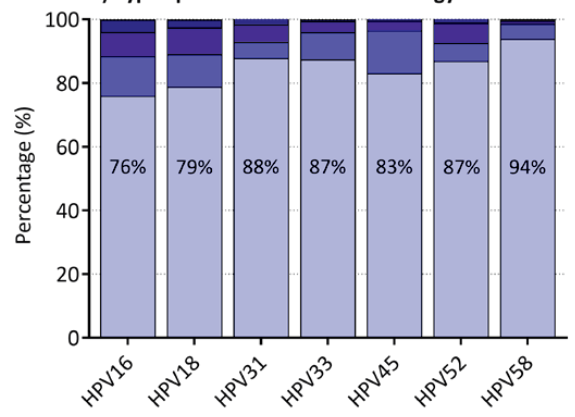

D) Combined HPV DNA and serology ${ }^{3}$

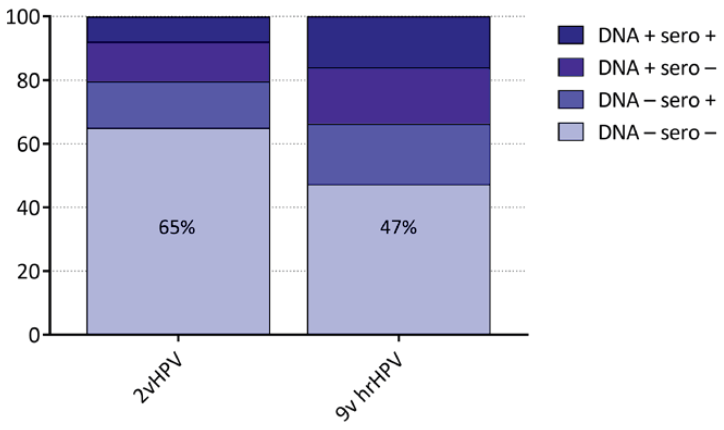

Figure 7.2. Percentage of MSM who were DNA negative (panel $A$ and $B$ ) and seronegative (panel $C$ and D) for the vaccine-targeted HPV types.

Note: For panel $A$ and $B$, all MSM with both swabs available were included $(n=455)$. For panel $C$ and $D$, all MSM with both swabs and serum available were included $(n=421)$. 2vHPV include HPV16/18; 4vHPV include HPV6/11/16/18; 9vHPV include HPV6/11/16/18/31/33/45/52/58. 9v hrHPV include HPV16/18/31/33/45/52/58. 1 Negative was defined as being negative for all the types. Positivity was defined as being positive for $\geq 1$ type. 2 DNA negative was defined as being negative in the penile as well as the anal swab, DNA positive was defined as being positive in $\geq 1 \mathrm{swab}$. Seropositivity was based on the predefined type-specific cut-off levels. 3 DNA negative was defined as being negative for all the types in the penile as well as the anal swab, DNA positive was defined as being positive for $\geq 1$ type in $\geq 1$ swab. Seronegative was defined as being seronegative for all types, seropositivity was defined as being seropositive for $\geq 1$ type based on the predefined type-specific cut-off levels. 
Also including serology, 76\% of the MSM were HPV16 negative (DNA negative in both swabs and seronegative) and 79\% were HPV18 negative (Figure 7.2C). For the other vaccinetargeted hrHPV, the percentage HPV DNA negative and seronegative was even higher and up to $94 \%$ for HPV58. Among MSM HPV16/18 and HPV16/18/31/33/45/52/58 DNA negative, $82 \%$ and $71 \%$ were also seronegative for the respective vaccine types. In total, $65 \%$ and $47 \%$ were HPV DNA negative and seronegative for HPV16/18 and HPV16/18/31/33/45/52/58 respectively (Figure 7.2D).

\section{HPV antibody concentration}

The HPV16 and HPV18 log antibody concentration increased both with age and number of lifetime sex partners $(p<.05)$. However, even in the highest categories of 23-to 24-year-olds and $\geq 40$ lifetime sex partners, the majority of the MSM were not seropositive (Figure 7.3). These patterns were comparable to the other hrHPV types (data not shown).

A) HPV16, by age

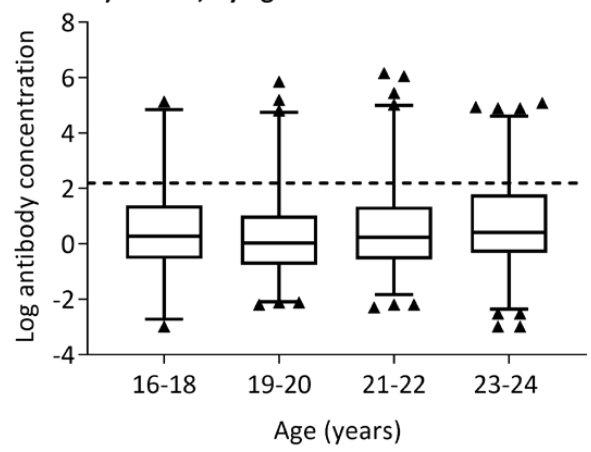

C) HPV16, by lifetime sex partners

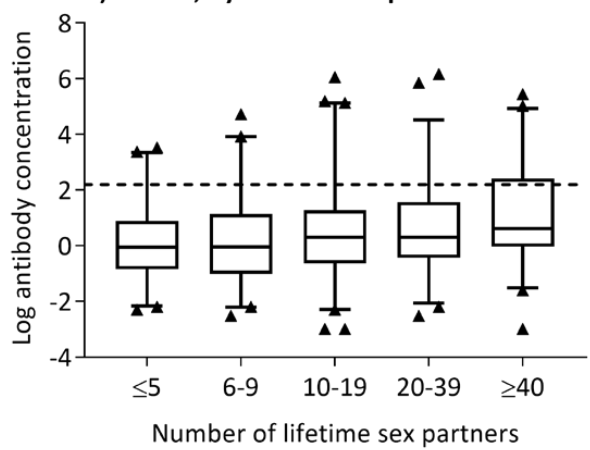

B) HPV18, by age

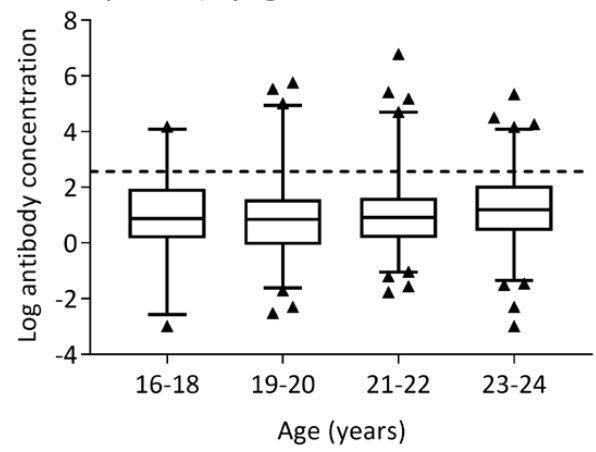

D) HPV18, by lifetime sex partners

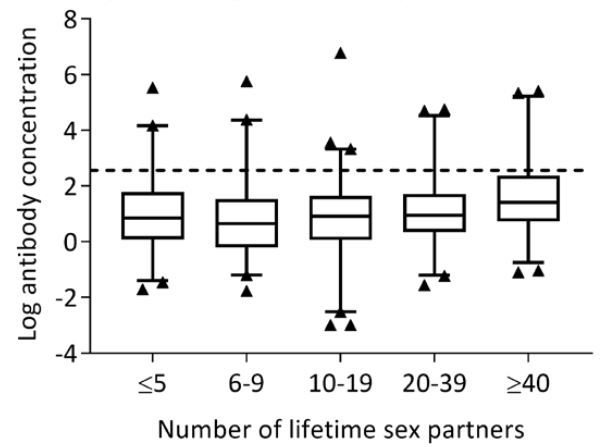

Figure 7.3. Log antibody concentration for HPV16 and HPV18 by age and number of lifetime sex partners.

Note: The dashed line represents the type-specific predefined cut-off level for seropositivity. The maximum number of reported lifetime sex partners was 900 . The error bars represent the 2.5 th and 97.5 th percentiles. 


\section{Discussion}

We assessed the scope for targeted HPV vaccination for sexually active MSM, by studying trends in the HPV prevalence over time and by studying the proportions (sero)negative for the various vaccine-targeted HPV types among young sexually active MSM who visited SHCS in the Netherlands. We did not discern trends for any of the vaccine types up to 8 years after the introduction of girls-only vaccination, and the majority of the MSM in our study population were HPV DNA negative and seronegative for the various vaccine types. Our study provides important baseline measurements in case male HPV vaccination will be implemented in the Netherlands. Moreover, because young MSM visiting SHCs are a natural target population for a selective vaccination program, our study may provide relevant input for countries considering targeted HPV vaccination for MSM.

We do acknowledge some limitations. First, MSM definition was based on selfidentification of sexual preference instead of behavior, because information on the sex of the sex partners was unavailable. Second, relatively small numbers of MSM were included per PASSYON study round, resulting in limited power to detect possible trends. Last, we only had data from young MSM up to 24 years of age with 3.5\% being HIV positive. We cannot extrapolate the results to older MSM visiting SHCs or MSM populations with a higher HIV prevalence. Whether prophylactic HPV vaccination of HIV positive MSM would be effective is still unclear; an RCT to study the vaccine efficacy among HIV infected adults aged 27 years or older was ended prematurely due to lack of effectiveness [27].

No significant declining trends were observed in the HPV16/18 prevalence among MSM in the aftermath of girls-only HPV16/18 vaccination. Given that a declining trend in the HPV16/18 prevalence was observed among heterosexual men in the PASSYON study (35\% decline in a 6-year period) [11], the lack of a noticeable trend among MSM in an 8-year period indicates that MSM are unlikely to benefit to a large extent from herd protection from girls-only vaccination. We did observe a declining trend in the AGW prevalence, presumably as a result of changes in the policy of the SHCs; persons with AGWs were more often referred to the general practitioner in recent years [28]. The declining trend is likely not a result of herd protection as the current vaccination program for girls does not include vaccination against HPV6/11, the main causes of AGWs [5].

In contrast to what is often assumed, our study shows that many young MSM visiting SHCs are HPV DNA negative and seronegative for the vaccine-targeted types, at least until the age of 24 years. For two-thirds of the MSM there was no evidence of current or past infection with both HPV16 and HPV18 at the penile as well as the anal site, suggesting that vaccination could still be beneficial. Note that this definition of negativity based on DNA and serostatus (negative for all measures for both HPV vaccine types) is more stringent than used in the RCT's per-protocol definition where negativity was defined as being DNA 
negative at the anatomic site and HPV type under study and seronegative for the HPV type under study $[18,19]$. Therefore, the proportion of MSM to experience vaccine-induced protection similar to the per-protocol efficacy demonstrated in RCTs will likely exceed twothirds of 16- to 24-year-old MSM. If someone is positive at 1 anatomical site, vaccination could possibly still prevent infections at the other site and if someone is positive for only 1 type included in the vaccine, vaccination could still be effective in preventing infections with the other type(s) [29]. All MSM were negative for at least one of the 2vHPV types at one or more anatomical sites, indicating that all MSM could derive at least partial benefit from vaccination. Focusing on HPV16, by far the most oncogenic type in men, $98 \%$ of the MSM were DNA negative at one or more anatomical sites. Moreover, although vaccination does not have a therapeutic effect on infections prevalent at the time of vaccination, it might still prevent future infections [30]. In contrast to women, where the peak of infection is before the mid-twenties, many MSM will keep being exposed and infected during many years of their lifetime [31]. Thus, as the risk of HPV acquisition does not diminish with age, vaccinating MSM at older age is still likely to be beneficial.

One of the inclusion criteria of the RCT where efficacy of HPV vaccination among MSM has been demonstrated, was having 1-5 lifetime sex partners [18, 19]. Of the MSM in our study, $80 \%$ had 6 or more partners and $20 \%$ even 40 or more. Despite these high numbers of partners, we observed a low type-specific (sero)prevalence for the various vaccine types. It could be that MSM without evidence of HPV exposure were previously infected but cleared the infection without seroconversion [32] or had a latent infection [33]. Prophylactic vaccination probably has no effect on latent infections and one could argue that MSM who previously cleared an infection are able to also clear a future infection, diminishing an additional benefit of vaccination. However, chance could play an important role in clearance [34] and build-up of (long lasting) natural immunity in men is not apparent from epidemiological data [35, 36]. Moreover, viral persistence and oncogenic potential might differ between different variants of the same HPV type [37]. Therefore, even if an MSM already cleared an infection, there is still a risk of acquiring a persistent infection in the future. Future research should focus on the role of latency and of clearance in relation to prior exposure, and how these factors could affect vaccine effectiveness (VE) when offering HPV vaccination to MSM with high numbers of lifetime sex partners.

The antibody concentration among MSM increased only slightly with age and number of lifetime partners; even among those with $\geq 40$ partners, the majority was not yet seropositive. The median HPV16- and HPV18-specific antibody concentrations among MSM with $\geq 40$ partners were also considerably lower than among vaccinated women in the PASSYON study (0.62 and 1.41, compared to 7.61 and $6.94 \mathrm{Ln} \mathrm{LU/mL,} \mathrm{respectively)} \mathrm{[38].} \mathrm{In}$ another study among MSM with a median age of 40 years, HPV16/18 antibody 
concentrations of over $6.2 \mathrm{Ln} \mathrm{LU} / \mathrm{mL}$ (i.e. $>500 \mathrm{LU} / \mathrm{mL}$ ) were not associated with a lower acquisition of anal or penile HPV infections over a 12-month period [35]. Vaccination could increase the antibody concentration of MSM, even among those previously exposed, up to levels affording protection against subsequent infections.

Taken together, even though the VE among MSM with a high number of sex partners is not clear-cut, it is likely that many young MSM visiting SHCs in the Netherlands could still benefit from HPV vaccination given the high proportions of HPV (sero)negativity for the relevant vaccine types and the likely limited build-up of natural immunity. This was also suggested in previous research [39-42]. Various modeling studies have indicated that targeted prophylactic vaccination for sexually active MSM could also be a (cost-) effective strategy on a population-level $[17,43]$, including a recent study using the context of the Netherlands [16]. The HPV16 prevalence in our study was in line with the predicted penile and anal HPV16 prevalence among MSM in that modeling study. However, projected reductions in HPV16 prevalence were strongly reduced if no effectiveness was assumed in MSM with prevalent infection at the time of vaccination. Because vaccination is most effective before HPV exposure and HPV positivity increases with lifetime number of partners, it is desirable to vaccinate MSM as early as possible. While our data suggest that vaccination might be effective for the population of 16- to 24-year-old MSM who visit a SHC, vaccination is preferably offered at the initial SHC visit. In our study, $13 \%$ reported never being tested for STIs indicating this was their first visit; the other MSM (87\%) had possibly visited the SHC in the past. HPV vaccination may also be beneficial for MSM not visiting SHCs; those are more difficult to target, but might be reached via the general practitioner or snowball sampling through MSM who do visit SHCs.

In conclusion, this study did not find evidence for declines in the prevalence of HPV vaccine types among MSM, indicating that they are unlikely to benefit to a large extent from herd effects from girls-only vaccination. Moreover this study shows that many young MSM visiting SHCs are HPV DNA negative and seronegative for the relevant vaccine types, indicating they could still benefit from HPV vaccination. Targeted MSM vaccination might be considered and SHCs could play an important role in promoting HPV vaccination to young MSM.

\section{References}

1. Chaturvedi AK. Beyond cervical cancer: burden of other HPV-related cancers among men and women. J Adolesc Health 2010; 46:S20-6.

2. European Medicines Agency (EMA). Cervarix: EPAR - Product Information. Available at: https://www.ema.europa.eu/en/medicines/human /EPAR/cervarix. Accessed 17-10-2019.

3. European Medicines Agency (EMA). Gardasil: EPAR - Product Information. Available at: https://www.ema.europa.eu/en/medicines/human /EPAR/gardasil. Accessed 17-10-2019. 
4. European Medicines Agency (EMA). Gardasil 9: EPAR - Product Information. Available at: https://www.ema.europa.eu/en/medicines/human /EPAR/gardasil-9. Accessed 17-10-2019.

5. Garland SM, et al. Natural history of genital warts: analysis of the placebo arm of 2 randomized phase III trials of a quadrivalent human papillomavirus (types $6,11,16$, and 18) vaccine. J Infect Dis 2009; 199:80514.

6. World Health Organization (WHO). Global market study: HPV vaccines. Available at: https://apps.who.int/iris/handle/10665/311275.

Accessed 16-12-2019.

7. Drolet $\mathrm{M}$, et al. Population-level impact and herd effects following the introduction of human papillomavirus vaccination programmes: updated systematic review and meta-analysis. Lancet 2019; 394:497-509.

8. WHO SAGE. Update on HPV vaccine introduction and programmatic perspectives. Available at: https://www.who.int/immunization/sage/meetings /2018/october/presentations_background_docs/en /index1.html. Accessed 17-01-2020.

9. Qendri V, et al. Ten years of HPV vaccination in the Netherlands: current evidence and future challenges in HPV-related disease prevention. Expert Rev Vaccines 2018; 17:1093-104.

10. Chow EPF, et al. Quadrivalent vaccine-targeted human papillomavirus genotypes in heterosexual men after the Australian female human papillomavirus vaccination programme: a retrospective observational study. Lancet Infect Dis 2017; 17:68-77.

11. Woestenberg PJ, et al. Assessment of herd effects among women and heterosexual men after girls-only HPV16/18 vaccination in the Netherlands: A repeated cross-sectional study. Int J Cancer 2019; 144:2718-27.

12. Chow EP, et al. Ongoing decline in genital warts among young heterosexuals 7 years after the Australian human papillomavirus (HPV) vaccination programme. Sex Transm Infect 2015; 91:214-9.

13. Machalek DA, et al. Anal human papillomavirus infection and associated neoplastic lesions in men who have sex with men: a systematic review and meta-analysis. Lancet Oncol 2012; 13:487-500.
14. Frisch $\mathrm{M}$, et al. Cancer in a population-based cohort of men and women in registered homosexual partnerships. Am J Epidemiol 2003; 157:966-72.

15. Daling JR, et al. Human papillomavirus, smoking, and sexual practices in the etiology of anal cancer. Cancer 2004; 101:270-80.

16. Bogaards JA, et al. Potential effectiveness of prophylactic HPV immunization for men who have sex with men in the Netherlands: A multi-model approach. PLoS Med 2019; 16:e1002756.

17. Zhang $L$, et al. Targeted human papillomavirus vaccination for young men who have sex with men in Australia yields significant population benefits and is cost-effective. Vaccine 2017; 35:4923-9.

18. Palefsky JM, et al. HPV vaccine against anal HPV infection and anal intraepithelial neoplasia. N Engl J Med 2011; 365:1576-85.

19. Giuliano AR, et al. Efficacy of quadrivalent HPV vaccine against HPV Infection and disease in males. N Engl J Med 2011; 364:401-11.

20. Xiridou $M$, et al. Hepatitis $B$ vaccination of men who have sex with men in the Netherlands: should we vaccinate more men, younger men or high-risk men? Sex Transm Infect 2013; 89:666-71.

21. Checchi M, et al. HPV vaccination of gay, bisexual and other men who have sex with men in sexual health and HIV clinics in England: vaccination uptake and attendances during the pilot phase. Sex Transm Infect 2019; 95:608-13.

22. Vriend $\mathrm{HJ}$, et al. Type-specific human papillomavirus infections among young heterosexual male and female STI clinic attendees. Sex Transm Dis 2012; 39:72-8.

23. Vriend $\mathrm{HJ}$, et al. Patterns of human papillomavirus DNA and antibody positivity in young males and females, suggesting a site-specific natural course of infection. PLoS One 2013; 8:e60696.

24. Scherpenisse M, et al. Seroprevalence of seven high-risk HPV types in The Netherlands. Vaccine 2012; 30:6686-93.

25. Szarewski A, et al. Efficacy of the human papillomavirus (HPV)-16/18 AS04-adjuvanted vaccine in women aged $15-25$ years with and without serological evidence of previous exposure to HPV16/18. Int J Cancer 2012; 131:106-16.

26. Castellsague $X$, et al. End-of-study safety, immunogenicity, and efficacy of quadrivalent HPV 
(types $6,11,16,18$ ) recombinant vaccine in adult women 24-45 years of age. Br J Cancer 2011; 105:2837.

27. Wilkin TJ, et al. A Randomized, PlaceboControlled Trial of the Quadrivalent Human Papillomavirus Vaccine in Human Immunodeficiency Virus-Infected Adults Aged 27 Years or Older: AIDS Clinical Trials Group Protocol A5298. Clin Infect Dis 2018; 67:1339-46.

28. Visser $M$, et al. Sexually transmitted infections in the Netherlands in 2016. Bitlhoven: RIVM, 2017. Nr: 2017-0003; DOI: 10.21945/RIVM-2017-0003.

29. Future II Study Group. Prophylactic efficacy of a quadrivalent human papillomavirus (HPV) vaccine in women with virological evidence of HPV infection. J Infect Dis 2007; 196:1438-46.

30. Swedish KA, et al. Prevention of recurrent highgrade anal neoplasia with quadrivalent human papillomavirus vaccination of men who have sex with men: a nonconcurrent cohort study. Clin Infect Dis 2012; 54:891-8.

31. Poynten IM, et al. Comparison of age-specific patterns of sexual behaviour and anal HPV prevalence in homosexual men with patterns in women. Sex Transm Infect 2016; 92:228-31.

32. Mooij SH, et al. HPV seroconversion following anal and penile HPV infection in HIV-negative and HIV-infected MSM. Cancer Epidemiol Biomarkers Prev 2014; 23:2455-61.

33. Twisk DE, et al. Detection of Incident Anal HighRisk Human Papillomavirus DNA in Men Who Have Sex With Men: Incidence or Reactivation? J Infect Dis 2018; 218:1018-26.

34. Ryser MD, et al. HPV clearance and the neglected role of stochasticity. PLoS Comput Biol 2015; 11:e1004113.

35. Mooij SH, et al. No evidence for a protective effect of naturally induced HPV antibodies on subsequent anogenital HPV infection in HIV-negative and HIV-infected MSM. J Infect 2014; 69:375-86.

36. Ranjeva SL, et al. Recurring infection with ecologically distinct HPV types can explain high prevalence and diversity. Proc Natl Acad Sci U S A 2017; 114:13573-8.

37. Gheit $T$, et al. Risks for persistence and progression by human papillomavirus type 16 variant lineages among a population-based sample of Danish women. Cancer Epidemiol Biomarkers Prev 2011; 20:1315-21.

38. Woestenberg PJ, et al. Bivalent Vaccine Effectiveness Against Type-Specific HPV Positivity: Evidence for Cross-Protection Against Oncogenic Types Among Dutch STI Clinic Visitors. J Infect Dis 2018; 217:213-22.

39. King EM, et al. Human papillomavirus DNA in men who have sex with men: type-specific prevalence, risk factors and implications for vaccination strategies. Br J Cancer 2015; 112:1585-93.

40. Cameron RL, et al. Baseline HPV prevalence in rectal swabs from men attending a sexual health clinic in Scotland: assessing the potential impact of a selective HPV vaccination programme for men who have sex with men. Sex Transm Infect 2020; 96:55-7. 41. Poynten IM, et al. Vaccine-preventable anal human papillomavirus in Australian gay and bisexual men. Papillomavirus Res 2017; 3:80-4.

42. Meites E, et al. Monitoring for Human Papillomavirus Vaccine Impact Among Gay, Bisexual, and Other Men Who Have Sex With Men-United States, 2012-2014. J Infect Dis 2016; 214:689-96.

43. Lin A, et al. Impact and Cost-effectiveness of Selective Human Papillomavirus Vaccination of Men Who Have Sex With Men. Clin Infect Dis 2017; 64:580-8. 


\section{Supplementary information to Chapter 7}

Supplementary Table 7.1. Characteristics of the MSM over the PASSYON study years.

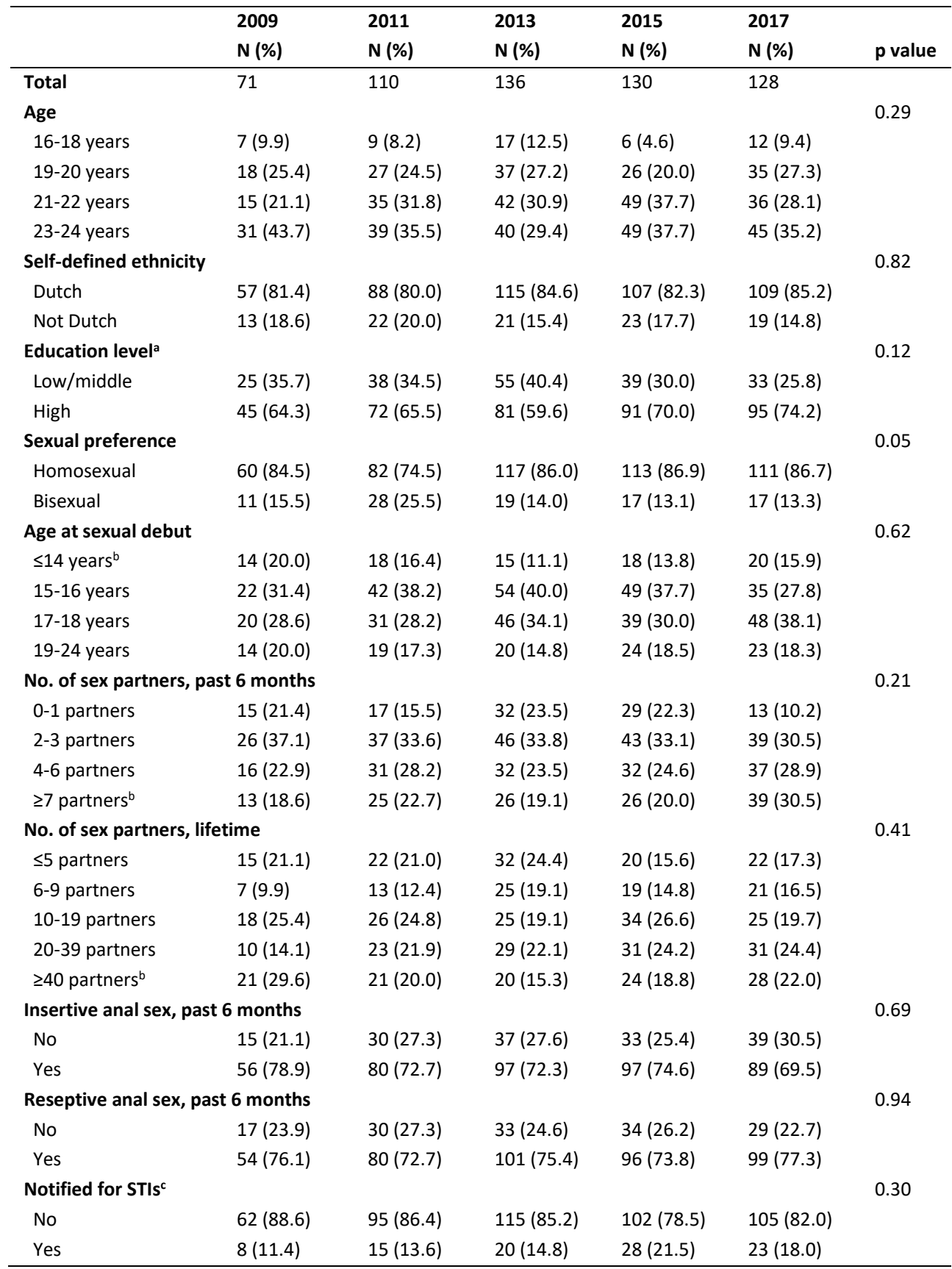


Supplementary Table 7.1. (Continued).

\begin{tabular}{|c|c|c|c|c|c|c|}
\hline & $\begin{array}{l}2009 \\
\text { N (\%) }\end{array}$ & $\begin{array}{l}2011 \\
\text { N (\%) }\end{array}$ & $\begin{array}{l}2013 \\
\text { N (\%) }\end{array}$ & $\begin{array}{l}2015 \\
\text { N (\%) }\end{array}$ & $\begin{array}{l}2017 \\
\text { N (\%) }\end{array}$ & p value \\
\hline STI-related symptoms ${ }^{c}$ & & & & & & 0.82 \\
\hline No & $54(77.1)$ & $88(80.0)$ & $104(77.0)$ & $100(76.9)$ & $105(82.0)$ & \\
\hline Yes & $16(22.9)$ & $22(20.0)$ & $31(23.0)$ & $30(23.1)$ & $23(18.0)$ & \\
\hline History of any STI & & & & & & 0.03 \\
\hline No & $40(57.1)$ & $55(50.0)$ & $63(46.3)$ & $58(44.6)$ & $53(41.4)$ & \\
\hline Yes & $24(34.3)$ & $44(40.0)$ & $45(33.1)$ & $61(46.9)$ & $55(43.0)$ & \\
\hline Never tested & $6(8.6)$ & $11(10.0)$ & $28(20.6)$ & $11(8.5)$ & $20(15.6)$ & \\
\hline Current STI, d & & & & & & 0.16 \\
\hline No & $55(78.6)$ & 89 (80.9) & $120(88.9)$ & $112(86.2)$ & $102(79.7)$ & \\
\hline Yes & $15(21.4)$ & 21 (19.1) & $15(11.1)$ & $18(13.8)$ & $26(20.3)$ & \\
\hline HIV infection ${ }^{c}$ & & & & & & 0.27 \\
\hline No & $69(98.6)$ & $108(98.2)$ & $130(95.6)$ & $121(93.8)$ & $74(98.7)$ & \\
\hline Yes & $1(1.4)$ & $2(1.8)$ & $6(4.4)$ & $8(6.2)$ & $1(1.3)$ & \\
\hline \multicolumn{6}{|c|}{ Condom use with casual partners, past 6 months ${ }^{e}$} & 0.33 \\
\hline Inconsistent & $10(14.1)$ & $24(21.8)$ & $28(20.6)$ & $27(20.8)$ & $35(27.3)$ & \\
\hline Consistent & $53(74.6)$ & $73(66.4)$ & $87(64.0)$ & $85(65.4)$ & $84(65.6)$ & \\
\hline No casual partners & $8(11.3)$ & $13(11.8)$ & $21(15.4)$ & $18(13.8)$ & $9(7.0)$ & \\
\hline
\end{tabular}

Totals vary because of missing values.

a. High educational level included school of higher general secondary education, pre-university education, university of applied sciences, and university. Low/middle educational level included all other levels of education.

b. The minimum reported age at sexual debut was 8 . The maximum number of reported sex partners in the past 6 months was 100 and lifetime 900.

c. Based on the visits at the SHC.

d. Including chlamydia, gonorrhea, and syphilis.

e. Inconsistent included reporting never, rarely and "sometimes I do, sometimes I do not" condom use. Consistent included reporting often or always condom use. 




\section{Part 3}

Direct vaccine effectiveness against anogenital warts 



\section{Chapter 8}

\section{No evidence for cross-protection of the HPV16/18 vaccine against HPV6/11 positivity in female STI clinic visitors}

Petra J. Woestenberg Audrey J. King Marianne A. B. van der Sande Robine Donken Suzan Leussink Fiona R. M. van der Klis Christian J. P. A. Hoebe Johannes A. Bogaards Birgit H. B. van Benthem 


\section{Abstract}

Introduction: Data from a vaccine trial and from post-vaccine surveillance in the United Kingdom have suggested that the bivalent HPV16/18 vaccine offers cross-protection against HPV6/11 and protection against anogenital warts (AGWs). We studied the effect of the bivalent vaccine on genital HPV6/11 positivity and AGWs in the Netherlands.

Methods: We included all vaccine-eligible women from the PASSYON study, a biennial cross-sectional study among 16- to 24-year-old sexually transmitted infection (STI) clinic attendants. Cervicovaginal self-swabs were analyzed for type-specific HPV and AGWs were diagnosed at the STI clinic. Prevalence of HPV6 and/or HPV11 and AGWs were compared between self-reported vaccinated and unvaccinated women by log-binomial regression analysis, adjusted for demographics and risk behavior.

Results: Of the 1,198 women included, $56 \%$ reported to be vaccinated at least once. Relative to unvaccinated women, the adjusted prevalence ratio (PR) for HPV6/11 was 1.03 (95\% confidence interval $[\mathrm{Cl}]$ 0.74-1.43) for women vaccinated at least once. The crude PR for AGWs was 0.67 (95\% $\mathrm{Cl}$ 0.22-2.07) for women vaccinated at least once. Adjustment did not change these results.

Discussion: We observed no cross-protective effect of the bivalent vaccine on genital HPV6/11 positivity and a non-significant partially protective effect on AGWs. 


\section{Introduction}

Human papillomavirus (HPV) is a sexually transmitted virus of which many different types exist. Types 16 and 18 are responsible for approximately $70 \%$ of all cervical cancers [1] and types 6 and 11 are responsible for approximately $90 \%$ of all anogenital warts (AGWs) [2].

Currently, 3 different vaccines against HPV are available: (A) the bivalent (2vHPV) vaccine (Cervarix ${ }^{\circledR}$ ) which protects against HPV types 16 and 18 [3]; (B) the quadrivalent (4vHPV) vaccine (Gardasil $\left.{ }^{\circledR}\right)$ which protects against HPV types $16,18,6$, and 11 [4]; (C) the nonavalent (9vHPV) vaccine (Gardasil9 ${ }^{\circledR}$ ) which protects against HPV types $16,18,6,11,31$, $33,45,52$, and 58 [5].

Although the $2 \mathrm{VHPV}$ vaccine is not indicated to prevent HPV6/11, some studies have suggested a cross-protective effect against HPV6/11 and a protective effect against AGWs. In post-hoc analyses of a 2 vHPV vaccine trial (PATRICIA trial) a vaccine efficacy of $35 \%$ ( $95 \%$ confidence interval [CI] 11\%-52\%) against 6-month persistent HPV6 and/or HPV11 infection was estimated among women who were HPV seronegative at baseline [6]. Moreover, data from post-vaccine surveillance in the United Kingdom suggested that the 2vHPV vaccine offers protection against AGWs. They observed a decline in AGWs among young girls and heterosexual men after introduction of the $2 \mathrm{vHPV}$ vaccine in 2008 , while such a decline was not observed among older women or men who have sex with men [7, 8]. The vaccine effectiveness (VE) against AGWs was calculated to be $34 \%$ (95\% Cl 29\%-38\%) [7]. In 2012, the United Kingdom switched to the 4vHPV vaccine in their national immunization program (NIP) and a further drop in AGWs is expected due to direct protection against HPV6/11.

The Netherlands is one of the few industrialized countries using the 2vHPV vaccine in the NIP. In 2009, there was a one-off catch-up campaign for girls born in 1993-1996 (12-16 years old). From 2010 onwards, girls are offered vaccination in the year they turn 13 years old, starting with birth cohort 1997 in 2010 [9]. The vaccination coverage for the catch-up cohorts was $52 \%$ [10]. The vaccination coverage of the routine vaccination was $56 \%$ for birth cohort 1997 and increased to 61\% for birth cohort 2001 [11].

From a public health perspective, monitoring of the effect of the HPV vaccination program includes its possible effect on non-vaccine HPV types. For this reason, a biennial cross-sectional study among young sexually transmitted infection (STI) clinic attendants was initiated in the Netherlands in 2009, the year of HPV vaccine introduction in the Netherlands.

In this paper, we explore the effects of the 2VHPV vaccine on genital HPV6 and/or HPV11 positivity and AGWs by comparing vaccinated and unvaccinated women with similar exposure, using data from STI clinics from 2011 to 2015. 


\section{Methods}

\section{Study design and population}

We used data from the PASSYON (PApillomavirus Surveillance among STI clinic YOungsters in the Netherlands) study. The study design is described in detail elsewhere [12]. Briefly, the PASSYON study is a biennial cross-sectional study among 16- to 24-year-old attendants of STI clinics located throughout the Netherlands. It started in 2009 and was repeated in 2011, 2013, and 2015 (Figure 8.1). Additional to the routine STI consultation, participants were asked to provide a genital self-swab for HPV testing and to fill-in a questionnaire including self-reported vaccination status. From participants who provided blood for routine STI diagnostics, serum was collected for HPV serology. AGWs were diagnosed during the routine STI consultation, based on clinical presentation. The Medical Ethical Committee of the University of Utrecht, the Netherlands approved this study (protocol number 08/397). Data were obtained using a unique code per person and all participants gave informed consent.

To study the effect of the 2vHPV vaccine on HPV6/11 and AGWs, we included from the PASSYON study years 2011, 2013, and 2015 all women born in 1993 or later, i.e. those who were eligible for vaccination in the catch-up campaign or the NIP (Figure 8.1).

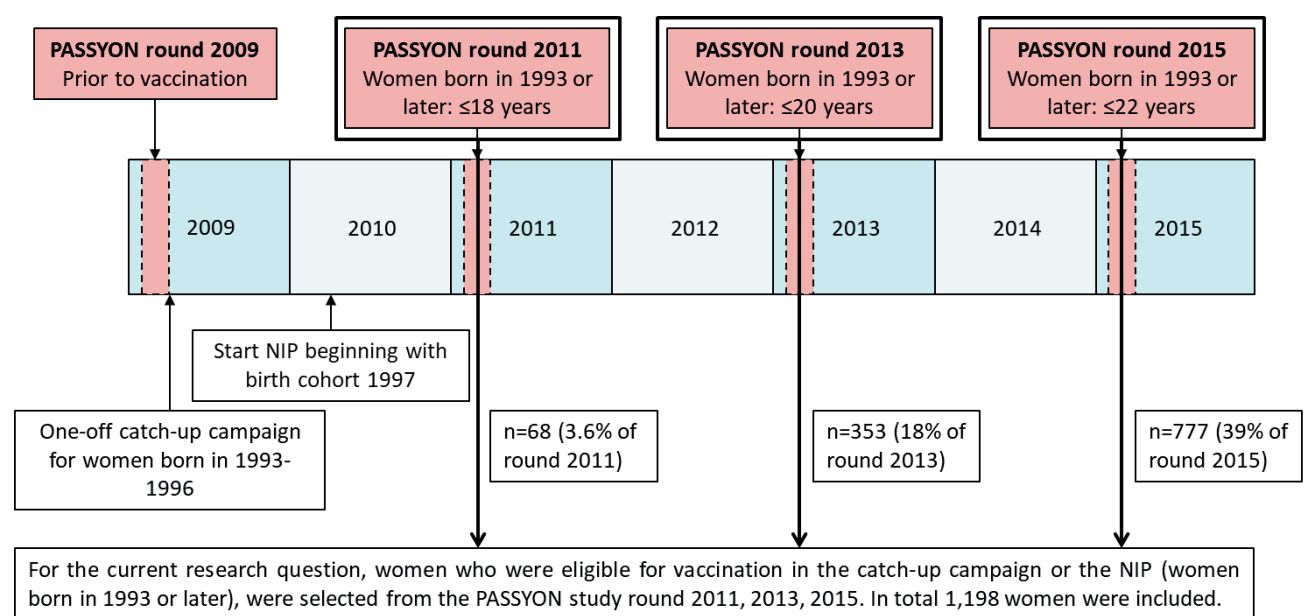

Figure 8.1. PASSYON study design and the study population selection. 


\section{Laboratory methods}

Swabs were stored at $-20^{\circ} \mathrm{C}$ until analyses [12]. DNA was extracted using the MagnaPure platform (Total Nucleic Acid Isolation Kit, Roche, the Netherlands) and eluted in 100microliter elution buffer. HPV DNA was amplified using the SPF10 primer set. Subsequently, HPV specific amplicons were detected using the DNA enzyme-linked immunoassay (HPVDEIA, DDL Diagnostics Laboratory, the Netherlands). Amplicons of positive samples were genotyped with Line probe assay (HPV-LiPA, DDL Diagnostics Laboratory, the Netherlands) which is able to detect 25 HPV types including types 6 and 11 [12].

Serum samples were stored at $-80^{\circ} \mathrm{C}$ until analyses [13]. HPV antibodies against L1 viruslike particles (VLPS) for 7 serotypes including types 16 and 18 were assessed using a multiplex immunoassay [14]. Cut-off levels for seropositivity were previously determined and were 9 Luminex Units (LU)/ml for HPV16 and 13 LU/ml for HPV18 [14].

\section{Statistical analyses}

First, we checked for differences in demographics and risk behavior between vaccinated and unvaccinated women. We compared women who reported to be vaccinated ( $\geq 1$ dose) with unvaccinated women and women who reported to be fully vaccinated ( 3 doses according guidelines prevailing at the time of vaccination for women eligible for this study [15]) with unvaccinated women. Women with an unknown vaccination status were analyzed separately.

We considered the demographic variables age, migration background, and education level. Migration background was based on (parental) country of birth. A woman was defined as native Dutch if both parents were born in the Netherlands [16]. Education level was selfreported and categorized as high and low/middle. High educational level included school of higher general secondary education, pre-university education, university of applied sciences, and university. Low/middle educational level included all other forms of education.

For risk behavior, we considered number of sex partners in the past 6 months, number of lifetime sex partners, age at sexual debut, history of STIs, condom use with casual partners in the past 6 months, hormonal contraceptives use, and current genital chlamydia or gonorrhea infection. The latter was based on diagnoses during the routine STI consultation. The other variables were self-reported and categorized (Table 8.1).

Prevalence of genital HPV6 and/or HPV11 positivity and AGWs were calculated by selfreported vaccination status. We calculated prevalence ratios (PR) using log-binomial regression analyses. For the outcome genital HPV6 and/or HPV11 positivity, we first calculated the crude PR. Second, we adjusted for age and demographic variables that were associated with vaccination status. Third, we further adjusted for risk behavior variables that were associated with vaccination status to measure the adjusted effect of vaccination 
on HPV6/11. For the outcome AGWs, we calculated the crude PR. Due to low numbers of AGWs in our study, we could not adjust for potential confounders all at once, so we adjusted for potential confounders in bivariable analyses only. Moreover, we described the genital HPV types that were found among women diagnosed with AGWs.

All analyses were performed using SAS version 9.3 (SAS Institute Inc., Cary, NC) with a significance level of $p<.05$. The records with missing data were excluded from the analyses, as these represented less than $5 \%$ of the study population.

\section{Sensitivity analyses}

HPV16 and HPV18 antibody concentrations were compared between self-reported vaccinated and unvaccinated women with serum available. Since all women vaccinated with the 2vHPV vaccine have an antibody response against HPV16 and HPV18 up to 9.4 years after vaccination [17], it is unlikely that vaccinated women are seronegative for HPV16/18. We repeated the analyses excluding women who reported to be vaccinated, but were seronegative for HPV16 or HPV18.

\section{Results}

\section{Study population}

In the PASSYON study, 1,198 women were eligible for HPV vaccination (born in 1993 or later) and included in the current study (Figure 8.1). Of these women, 666 (56\%) reported to be vaccinated at least once and $450(38 \%)$ reported not to be vaccinated. The remaining did not know or did not report their vaccination status. 467 women (39\%) reported to be fully vaccinated. Of the women who reported to be vaccinated at least once, the majority (94\%) was vaccinated during the catch-up campaign (birth cohorts 1993-1996). Of the total 1,198 women included, 1,168 women (97.5\%) had a genital swab taken and 1,193 women (99.6\%) provided information about the STI consult including diagnoses of AGWs.

In Table 8.1, the characteristics of the study population are presented, stratified by vaccination status. There were some differences between vaccinated and unvaccinated women. Vaccinated women were more often native Dutch and highly educated. They less often had a history of STIs and used hormonal contraceptives more often. Women vaccinated at least once had more partners in the past 6 months and were older at sexual debut, but these factors did not differ statistically significant between fully vaccinated and unvaccinated women. 
Table 8.1. Characteristics of the study population and a comparison between vaccinated and unvaccinated women.

\begin{tabular}{|c|c|c|c|c|c|c|c|}
\hline & \multirow{3}{*}{$\begin{array}{l}\text { Total } \\
\text { N (\%) }\end{array}$} & \multirow{3}{*}{$\begin{array}{l}\text { Unvaccinated } \\
\mathrm{N}(\%)\end{array}$} & \multicolumn{2}{|c|}{ Vaccinated ( $\geq 1$ dose) } & \multicolumn{2}{|c|}{ Fully vaccinated $^{\mathrm{a}}$} & \multirow{3}{*}{$\begin{array}{l}\text { Unknown } \\
\mathrm{N}(\%)\end{array}$} \\
\hline & & & $\mathbf{N}(\%)$ & p & $\mathbf{N}(\%)$ & p & \\
\hline & & & & value $^{\text {b }}$ & & value $^{\text {b }}$ & \\
\hline Total & 1198 & 450 & 666 & & 467 & & 82 \\
\hline Age & & & & 0.47 & & 0.69 & \\
\hline $16-18$ years & $353(29.5)$ & $138(30.7)$ & $191(28.7)$ & & 149 (31.9) & & $24(29.3)$ \\
\hline $19-22$ years & $845(70.5)$ & $312(69.3)$ & 475 (71.3) & & $318(68.1)$ & & $58(70.7)$ \\
\hline \multicolumn{2}{|c|}{ Migration background } & & & $<.01$ & & $<.01$ & \\
\hline Native Dutch & $938(78.3)$ & $323(71.8)$ & $557(83.6)$ & & $397(85.0)$ & & $58(70.7)$ \\
\hline Not native Dutch & $255(21.3)$ & $125(27.8)$ & $107(16.1)$ & & $69(14.8)$ & & $23(28.0)$ \\
\hline Education level & & & & $<.01$ & & $<.01$ & \\
\hline Low/middle & $386(32.2)$ & $176(39.1)$ & $174(26.1)$ & & $126(27.0)$ & & 36 (43.9) \\
\hline High & $809(67.5)$ & $274(60.9)$ & $491(73.7)$ & & 341 (73.0) & & $44(53.7)$ \\
\hline \multicolumn{2}{|c|}{ No. of sex partners, past 6 months } & & & 0.01 & & 0.08 & \\
\hline 0-1 partners & $349(29.1)$ & $151(33.6)$ & $169(25.4)$ & & $127(27.2)$ & & $29(35.4)$ \\
\hline 2-3 partners & $578(48.2)$ & $207(46.0)$ & 339 (50.9) & & $245(52.5)$ & & $32(39.0)$ \\
\hline$\geq 4$ partners & $271(22.6)$ & $92(20.4)$ & $158(23.7)$ & & $95(20.3)$ & & $21(25.6)$ \\
\hline \multicolumn{2}{|c|}{ No. of sex partners, lifetime } & & & 0.23 & & 0.95 & \\
\hline$\leq 3$ partners & $317(26.5)$ & $130(28.9)$ & $164(24.6)$ & & $132(28.3)$ & & $23(28.0)$ \\
\hline 4-6 partners & 370 (30.9) & $140(31.1)$ & $215(32.3)$ & & $150(32.1)$ & & $15(18.3)$ \\
\hline$\geq 7$ partners & $486(40.6)$ & $172(38.2)$ & $280(42.0)$ & & $180(38.5)$ & & $34(41.5)$ \\
\hline \multicolumn{2}{|l|}{ Age at sexual debut } & & & 0.04 & & 0.15 & \\
\hline$\leq 14$ years & $210(17.5)$ & $93(20.7)$ & $102(15.3)$ & & $76(16.3)$ & & $15(18.3)$ \\
\hline $15-16$ years & $616(51.4)$ & $227(50.4)$ & $346(52.0)$ & & $240(51.4)$ & & $43(52.4)$ \\
\hline$\geq 17$ years & $353(29.5)$ & $124(27.6)$ & $213(32.0)$ & & $148(31.7)$ & & $16(19.5)$ \\
\hline \multicolumn{2}{|l|}{ History of any STI } & & & 0.02 & & $<.01$ & \\
\hline No & $625(52.2)$ & $221(49.1)$ & $373(56.0)$ & & $273(58.5)$ & & $31(37.8)$ \\
\hline Yes & $269(22.5)$ & $116(25.8)$ & $129(19.4)$ & & $78(16.7)$ & & $24(29.3)$ \\
\hline Never tested & $296(24.7)$ & $111(24.7)$ & $162(24.3)$ & & $114(24.4)$ & & $23(28.0)$ \\
\hline \multicolumn{3}{|c|}{ Current genital chlamydia/gonorrhea infection } & & 0.93 & & 0.57 & \\
\hline No & $980(81.8)$ & $366(81.3)$ & $547(82.1)$ & & $389(83.3)$ & & $67(81.7)$ \\
\hline Yes & $212(17.7)$ & $80(17.8)$ & $118(17.7)$ & & $77(16.5)$ & & $14(17.1)$ \\
\hline \multicolumn{3}{|c|}{ Condom use with casual partners, past 6 months ${ }^{c}$} & & 0.22 & & 0.47 & \\
\hline Inconsistent & $567(47.3)$ & $204(45.3)$ & $322(48.3)$ & & $221(47.3)$ & & $41(50.0)$ \\
\hline Consistent & $356(29.7)$ & $134(29.8)$ & $207(31.1)$ & & $145(31.0)$ & & $15(18.3)$ \\
\hline $\begin{array}{l}\text { No casual } \\
\text { partners }\end{array}$ & $267(22.3)$ & $111(24.7)$ & $135(20.3)$ & & $99(21.2)$ & & $21(25.6)$ \\
\hline \multicolumn{3}{|c|}{ History of using hormonal contraceptives } & & 0.01 & & 0.01 & \\
\hline No & $52(4.3)$ & $26(5.8)$ & $18(2.7)$ & & $12(2.6)$ & & $8(9.8)$ \\
\hline Yes & 1125 (93.9) & $416(92.4)$ & 641 (96.2) & & 448 (95.9) & & $68(82.9)$ \\
\hline
\end{tabular}




\section{HPV6/11}

In total, 122 women (10\%) were positive for HPV6, 18 women (1.5\%) for HPV11, and 2 women $(0.2 \%)$ for both. Among unvaccinated women, $12 \%$ was positive for genital HPV6 and/or HPV11. Among women who reported to be vaccinated ( $\geq 1$ dose), this was $13 \%$ (Figure 8.2). After adjustment for demographics and risk behavior, the PR of women vaccinated ( $\geq 1$ dose) was 1.03 (95\% $\mathrm{Cl} 0.74-1.43$ ) relative to unvaccinated women (Table 8.2). Of the fully vaccinated women, $11 \%$ was positive for genital HPV6 and/or HPV11. Comparing fully vaccinated women with unvaccinated women, the PR was $0.91(95 \% \mathrm{Cl}$ 0.63-1.31) adjusted for demographics and risk behavior.

\section{Anogenital warts}

In total, only $13(1.1 \%)$ out of 1,193 vaccine-eligible women with information of the STI consult were diagnosed with AGWs. Among unvaccinated women, 1.3\% were diagnosed with AGWs and among vaccinated women ( $\geq 1$ dose) this was $0.9 \%$ (Figure 8.2 ), resulting in a PR of 0.67 (95\% Cl 0.22-2.07) (Table 8.2). Adjustment for demographics or risk behavior in bivariable analyses did not lead to other PRs; ranging from 0.66 adjusted for education level to 0.69 adjusted for number of partners in the past 6 months. Among fully vaccinated women, $0.9 \%$ were diagnosed with AGWs, giving a PR of $0.64(95 \% \mathrm{Cl} 0.18-2.25)$ relative to unvaccinated women. Adjustment resulted in PRs ranging from 0.63 adjusted for hormonal contraceptives use to 0.65 adjusted for number of partners in the past 6 months.

A) HPV6/11

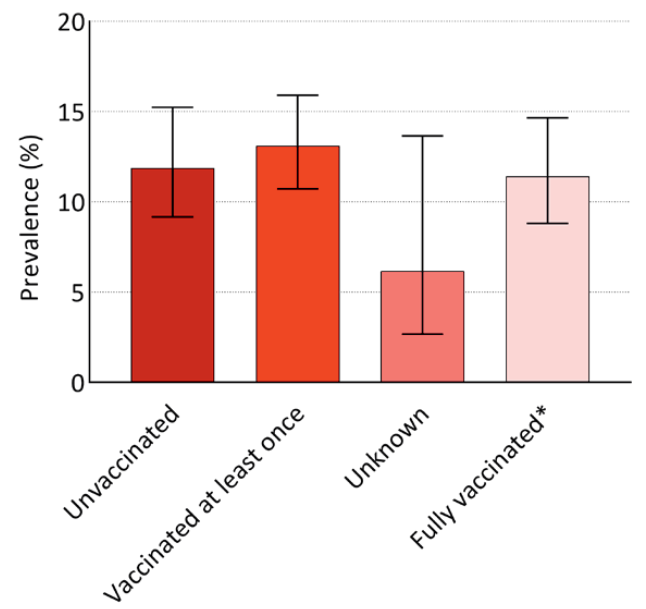

\section{B) Anogenital warts}

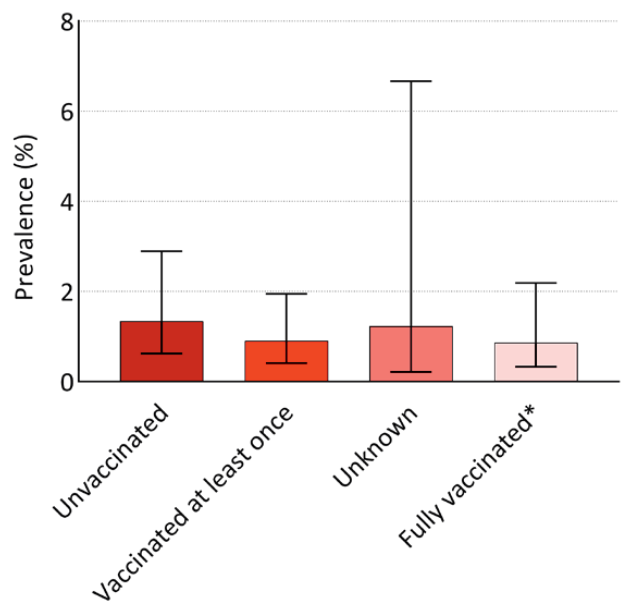

Figure 8.2. A) prevalence of genital HPV6 and/or HPV11 positivity and B) anogenital warts, by vaccination status.

Note: * Fully vaccinated women reported to be vaccinated 3 times. Note the different scale of the $y$-axes. 
Table 8.2. Prevalence and prevalence ratios of genital HPV6 and/or HPV11 positivity and anogenital warts by vaccination status.

\begin{tabular}{|c|c|c|c|c|c|}
\hline & $\mathbf{N}$ & $\begin{array}{l}\text { n Events } \\
\text { (\%) }\end{array}$ & $\begin{array}{l}\text { Crude PR } \\
(95 \% \mathrm{CI})\end{array}$ & $\begin{array}{l}\text { Adjusted PR } \\
(95 \% \mathrm{Cl})^{b}\end{array}$ & $\begin{array}{l}\text { Adjusted PR } \\
(95 \% \mathrm{Cl})^{c}\end{array}$ \\
\hline \multicolumn{6}{|c|}{ Genital HPV6 and/or HPV11 } \\
\hline Unvaccinated & 438 & 52 (11.9) & Reference & Reference & Reference \\
\hline Vaccinated ( $\geq 1$ dose) & 649 & 85 (13.1) & $1.10(0.80-1.52)$ & $1.04(0.75-1.45)$ & $1.03(0.74-1.43)$ \\
\hline Fully vaccinated ${ }^{a}$ & 456 & $52(11.4)$ & $0.96(0.67-1.38)$ & $0.91(0.63-1.31)$ & $0.91(0.63-1.31)$ \\
\hline Unknown & 81 & $5(6.2)$ & $0.52(0.21-1.26)$ & $0.43(0.16-1.16)$ & $0.45(0.17-1.21)$ \\
\hline \multicolumn{6}{|l|}{ Anogenital warts } \\
\hline Unvaccinated & 447 & $6(1.3)$ & Reference & - & - \\
\hline Vaccinated ( $\geq 1$ dose) & 665 & $6(0.9)$ & $0.67(0.22-2.07)$ & - & - \\
\hline Fully vaccinated ${ }^{a}$ & 466 & $4(0.9)$ & $0.64(0.18-2.25)$ & - & - \\
\hline Unknown & 81 & $1(1.2)$ & $0.92(0.11-7.54)$ & - & - \\
\hline
\end{tabular}

Table 8.3 shows the genital HPV types that were found among women diagnosed with AGWs, by vaccination status. All women with AGWs were HPV positive and all 6 vaccinated women were positive for HPV6. Of the 6 unvaccinated women, 3 were positive for HPV6. HPV11 was not found among women diagnosed with AGWs.

Table 8.3. Genital HPV types of women with anogenital warts, by vaccination status.

\begin{tabular}{lll}
\hline Sample & Self-reported vaccination status & Genital HPV types isolated \\
\hline 1 & Unvaccinated & $6,18,31,53,56,59$ \\
2 & Unvaccinated & $6,31,33,52$ \\
3 & Unvaccinated & $6,39,51,56$ \\
4 & Unvaccinated & 16 \\
5 & Unvaccinated $_{6}$ & 31 \\
6 & Unvaccinated $_{7}$ & 51 \\
8 & Fully vaccinated & \\
9 & Fully vaccinated & \\
10 & Fully vaccinated & $6,51,66$ \\
11 & Fully vaccinated & 6,51 \\
12 & Vaccinated at least once $_{13}$ & 6 \\
\hline
\end{tabular}

a. Fully vaccinated women reported to be vaccinated 3 times. 


\section{Sensitivity analyses}

Of the total study population, $43 \%$ had serum available for HPV antibody testing. Of the 268 women who reported to be vaccinated ( $\geq 1$ dose) with serum available, only 11 (4.1\%) were seronegative for either HPV16 or HPV18. Excluding these 11 women from the analyses did not change the results. Of the 194 women who reported to be fully vaccinated with serum available, 8 (4.1\%) were seronegative for HPV16 or HPV18. Excluding these 8 women from the analyses comparing fully vaccinated with unvaccinated women did also not change the results (Supplementary Table 8.1).

\section{Discussion}

We estimated the effect of the HPV16/18 vaccine on genital HPV6 and/or HPV11 positivity and AGWs among vaccine-eligible women attending STI clinics in the Netherlands. We observed no cross-protective effect of the 2VHPV vaccine on genital HPV6/11 positivity and a non-significant partially protective effect on AGWs.

To our knowledge, this is the first study to directly estimate the protective effect of the 2vHPV vaccine on both genital HPV6/11 positivity and AGWs among vaccine-eligible women. However, we do acknowledge some limitations. First, we had very low numbers of AGWs in our study population leading to insufficient power to robustly assess an effect of vaccination on AGW diagnoses. The low number of AGWs is partly because most AGWs in the Netherlands are diagnosed by the general practitioner [18]. Another reason is that among women, physical examination and thus diagnosis of AGWs is performed in case of reported symptoms only. Likely, some diagnoses of AGWs were missed in our study and the true prevalence of AGWs was probably higher. While it is unlikely that the effect estimate is biased for this reason, as the rate of underdiagnoses is probably not related to vaccination status, the low occurrence of AGWs influenced the precision of our effect estimate. Second, vaccination status was self-reported, which is prone to recall bias. However, only $4 \%$ of the women who reported to be vaccinated were seronegative for HPV16 or HPV18. We performed sensitivity analyses, excluding women who likely incorrectly reported to be vaccinated, but this did not change the results. Third, there were some differences between vaccinated and unvaccinated women in our study. Vaccinated women were often native Dutch and highly educated, which is in line with earlier findings [19]. Moreover, we observed some differences in sexual behavior between vaccinated and unvaccinated women. Although there is no evidence that HPV vaccination leads to other/higher sexual risk behavior [20], it is possible that sexual behavior was indirectly associated with vaccination, meaning that girls who chose to get vaccinated were a different population than girls who chose not to get vaccinated, and therefore developed another sexual risk 
profile. While we adjusted for demographics and risk behavior, we cannot rule out residual confounding. Last, our study population consisted of women who visited the STI clinic (suggesting high sexual risk behavior) and who were primarily vaccinated during the catchup campaign. Based on the reported age at sexual debut in the questionnaire and the scheduled vaccinations by birth cohort [19], about $7.7 \%-25 \%$ of the vaccinated women in our study were possibly already exposed to HPV before vaccination.

There is a possibility that the 2VHPV vaccine offers protection against AGWs, whilst not providing cross-protective effectiveness against HPV6/11. In many AGWs, multiple HPV types have been found, including types 16 and 18 [21, 22]. HPV16/18 could play a role in the development of AGWs, either indirectly by interaction with HPV6/11 or directly. Indeed, in some AGWs HPV16 was pointed out to be the probable type causing the wart $[23,24]$. Although surface swabbing does not necessarily indicate the causative HPV type [23], in our study we found 1 women with AGWs who was positive for HPV16 only.

HPV6 and HPV11 (both from the $\alpha-10$ species), are phylogenetically not closely related to the vaccine types HPV16 ( $\alpha-9$ species) and HPV18 ( $\alpha-7$ species) [25]. Nevertheless, in the PATRICIA trial, a vaccine efficacy of the $2 \mathrm{VHPV}$ vaccine of $35 \%$ against 6 -month persistent HPV6/11 infection was reported among women who were HPV seronegative at baseline [6]. Adjusted for demographics and risk behavior, we calculated a PR of 0.91 (95\% Cl 0.63-1.31) against HPV6/11 positivity for fully vaccinated women. This estimate corresponds to a VE of $9 \%(95 \% \mathrm{Cl}-31 \%$ to $37 \%)$ [26]. Although this suggests no effect, the confidence interval includes the $35 \%$ efficacy reported from the vaccine trial. In our cross-sectional study, we did not have information on duration of infection hampering a direct comparison.

The cross-protective effect of the 2vHPV vaccine on HPV6/11 positivity has so far not been replicated in post-vaccine surveillance studies. In England, where the 2vHPV vaccine was used until 2012, the prevalence of HPV6 and/or HPV11 among 16- to 18-year-old women increased from $5.8 \%$ in the pre-vaccination period to $8.3 \%$ in the post-vaccination period [27]. In Scotland, where the 2vHPV vaccine was also used until 2012, there were no differences in HPV6/11 positivity among vaccinated and unvaccinated women who underwent their first cervical screening [28]. However, these analyses were unadjusted for confounders and not all women were eligible for vaccination.

Interestingly, we calculated a crude PR of 0.64 for AGWs for fully vaccinated women, corresponding to a VE of $36 \%$. Although non-significant, this point estimate is almost identical to the VE against AGWs calculated by Howell-Jones and colleagues in an ecological study in the United Kingdom (34\%) [7]. However, in a study conducted in the Czech Republic, no effect of the 2vHPV vaccine on AGWs prevalence was observed among 16- to 40-year-old women [29]. 
In conclusion, our study neither supports nor excludes cross-protection from the 2vHPV vaccine against HPV6/11. We also could not confirm a protective effect of the 2vHPV vaccine against AGWs, 6 years after introduction of vaccination. For a more definite outcome, larger and longer duration studies would be needed. We are currently working on a research proposal to investigate the effect of the $2 \mathrm{VHPV}$ vaccine on AGWs using data from a large general practice network in the Netherlands where more diagnoses of AGWs are anticipated.

\section{References}

1. de Sanjose $S$, et al. Human papillomavirus genotype attribution in invasive cervical cancer: a retrospective cross-sectional worldwide study. Lancet Oncol 2010; 11:1048-56.

2. Garland SM, et al. Natural history of genital warts: analysis of the placebo arm of 2 randomized phase III trials of a quadrivalent human papillomavirus (types $6,11,16$, and 18) vaccine. J Infect Dis 2009; 199:80514.

3. Paavonen J, et al. Efficacy of human papillomavirus (HPV)-16/18 AS04-adjuvanted vaccine against cervical infection and precancer caused by oncogenic HPV types (PATRICIA): final analysis of a doubleblind, randomised study in young women. Lancet 2009; 374:301-14.

4. Future I/II Study Group. Four year efficacy of prophylactic human papillomavirus quadrivalent vaccine against low grade cervical, vulvar, and vaginal intraepithelial neoplasia and anogenital warts: randomised controlled trial. BMJ 2010; 341:c3493.

5. Joura EA, et al. A 9-valent HPV vaccine against infection and intraepithelial neoplasia in women. $\mathrm{N}$ Engl J Med 2015; 372:711-23.

6. Szarewski A, et al. Efficacy of the HPV-16/18 AS04adjuvanted vaccine against low-risk HPV types (PATRICIA randomized trial): an unexpected observation. J Infect Dis 2013; 208:1391-6.

7. Howell-Jones R, et al. Declining Genital Warts in Young Women in England Associated With HPV 16/18 Vaccination: An Ecological Study. J Infect Dis 2013; 208:1397-403.

8. Canvin $\mathrm{M}$, et al. Decline in genital warts diagnoses among young women and young men since the introduction of the bivalent HPV (16/18) vaccination programme in England: an ecological analysis. Sex Transm Infect 2017; 93:125-8.

9. de Melker HE, et al. [Introductie van vaccinatie tegen baarmoederhalskanker]. NTvG 2009; 153:65861.

10. van Lier EA, et al. [Vaccinatiegraad Rijksvaccinatieprogramma Nederland: Verslagjaar 2011]. Bilthoven: RIVM, 2011. Nr: 210021014/2011. 11. van Lier EA, et al. [Vaccinatiegraad Rijksvaccinatieprogramma Nederland: Verslagjaar 2011]. Bilthoven: RIVM, 2016. Nr: 2016-0064.

12. Vriend $\mathrm{HJ}$, et al. Type-specific human papillomavirus infections among young heterosexual male and female STI clinic attendees. Sex Transm Dis 2012; 39:72-8.

13. Vriend $\mathrm{HJ}$, et al. Patterns of human papillomavirus DNA and antibody positivity in young males and females, suggesting a site-specific natural course of infection. PLoS One 2013; 8:e60696.

14. Scherpenisse $M$, et al. Seroprevalence of seven high-risk HPV types in The Netherlands. Vaccine 2012; 30:6686-93.

15. Donken R, et al. Human papillomavirus (HPV) infection. In: Schurink-van 't Klooster TM, de Melker $\mathrm{HE}$, eds. The National Immunisation Programme in the Netherlands - Surveillance and developments in 2014-2015. Bilthoven: RIVM, 2015:127-38.

16. Woestenberg PJ, et al. Comparison of STI-related consultations among ethnic groups in the Netherlands: an epidemiologic study using electronic records from general practices. BMC Fam Pract 2015; 16:70. 
17. Naud PS, et al. Sustained efficacy, immunogenicity, and safety of the HPV-16/18 AS04adjuvanted vaccine: final analysis of a long-term follow-up study up to 9.4 years post-vaccination. Hum Vaccin Immunother 2014; 10:2147-62.

18. Van den Broek IVF, et al. Sexually tranmitted infections in the Netherlands in 2015. Bilthoven: National Institute for Public Health and the Environment, 2016. Nr: 2016-0027.

19. Rondy $M$, et al. Determinants for HPV vaccine uptake in the Netherlands: A multilevel study. Vaccine 2010; 28:2070-5.

20. Kasting $M L$, et al. Tempest in a teapot: $A$ systematic review of HPV vaccination and risk compensation research. Hum Vaccin Immunother 2016; 12:1435-50.

21. Aubin $F$, et al. Human papillomavirus genotype distribution in external acuminata condylomata: a Large French National Study (EDiTH IV). Clin Infect Dis 2008; 47:610-5.

22. Sturegard E, et al. Human papillomavirus typing in reporting of condyloma. Sex Transm Dis 2013; 40:123-9.

23. Hawkins MG, et al. Detection of specific HPV subtypes responsible for the pathogenesis of condylomata acuminata. Virol J 2013; 10:137.
24. Ball SL, et al. Analyses of human papillomavirus genotypes and viral loads in anogenital warts. J Med Virol 2011; 83:1345-50.

25. Harari A, et al. Human papillomavirus genomics: past, present and future. Curr Probl Dermatol 2014; 45:1-18.

26. Halloran ME, et al. Design and Analysis of Vaccine Studies. New York: Springer, 2010 (Gail M, Krickeberg K, Samet J, Tsiatis A, Wong W, eds. Statistics for Biology and Health).

27. Mesher $D$, et al. Continuing reductions in HPV $16 / 18$ in a population with high coverage of bivalent HPV vaccination in England: an ongoing crosssectional study. BMJ Open 2016; 6:e009915.

28. Cameron RL, et al. Human Papillomavirus Prevalence and Herd Immunity after Introduction of Vaccination Program, Scotland, 2009-2013. Emerg Infect Dis 2016; 22:56-64.

29. Petras $M$, et al. Impact of quadrivalent human papillomavirus vaccine in women at increased risk of genital warts burden: Population-based crosssectional survey of Czech women aged 16 to 40 years. Vaccine 2015; 33:6264-7. 


\section{Supplementary information to Chapter 8}

Supplementary Table 8.1. Sensitivity analyses: prevalence ratios of genital HPV6 and/or HPV11 positivity and anogenital warts by vaccination status, excluding women reported to be vaccinated without an HPV16 or HPV18 antibody response.

\begin{tabular}{|c|c|c|c|}
\hline & Crude PR $(95 \% \mathrm{Cl})$ & aPR $(95 \% \mathrm{Cl})^{b}$ & aPR $(95 \% \mathrm{Cl})^{c}$ \\
\hline \multicolumn{4}{|c|}{ Genital HPV6 and/or HPV11 } \\
\hline Unvaccinated & Reference & Reference & Reference \\
\hline Vaccinated ( $\geq 1$ dose $)^{\text {a }}$ & $1.07(0.77-1.48)$ & $1.00(0.72-1.40)$ & $0.99(0.71-1.38)$ \\
\hline Fully vaccinated ${ }^{a}$ & $0.92(0.64-1.33)$ & $0.86(0.60-1.25)$ & $0.86(0.59-1.26)$ \\
\hline Unknown & $0.52(0.21-1.26)$ & $0.43(0.16-1.16)$ & $0.46(0.17-1.23)$ \\
\hline \multicolumn{4}{|l|}{ Anogenital warts } \\
\hline Unvaccinated & Reference & - & - \\
\hline Vaccinated ( $\geq 1$ dose $)^{a}$ & $0.68(0.22-2.11)$ & - & - \\
\hline Fully vaccinated ${ }^{a}$ & $0.65(0.18-2.29)$ & & \\
\hline Unknown & $0.92(0.11-7.54)$ & - & - \\
\hline
\end{tabular}

a. Women who were seronegative for HPV16 or HPV18 were excluded from the analyses. Fully vaccinated reported to be vaccinated 3 times.

b. Corrected for demographic variables (age, migration background, and education level).

c. Corrected for demographic variables (age, migration background, and education level) and risk behavior (no. of sex partners in the past 6 months, age at sexual debut, history of any STI, and history of hormonal contraceptives use). 




\title{
Chapter 9
}

\section{Partial protective effect of bivalent HPV16/18 vaccination against anogenital warts in a large cohort of Dutch primary care patients}

\author{
Petra J. Woestenberg \\ Alejandra E. Guevara Morel \\ Johannes A. Bogaards \\ Mariëtte Hooiveld \\ Tessa M. Schurink-van 't Klooster \\ Christian J. P. A. Hoebe \\ Marianne A. B. van der Sande \\ Birgit H. B. van Benthem
}




\section{Abstract}

Introduction: There is ongoing debate about the possible protective effect of the bivalent human papillomavirus (2vHPV) vaccine, targeting oncogenic types HPV16/18, against anogenital warts (AGWs), commonly attributed to HPV6/11. We performed a retrospective registry-based open cohort study to assess the effect of $2 \mathrm{VHPV}$ vaccination on AGWs.

Methods: We linked general practice (i.e. primary care) data from women born between 1993 and 2002, who had been eligible for HPV vaccination in the Netherlands, to the Dutch national immunization registry on an individual level. Women were followed until their first AGW diagnosis or end of follow-up. Adjusted incidence rate ratios (aIRRs) were estimated using Poisson regression with vaccination status as a time-dependent exposure.

Results: We linked data of 96,468 women with in total 328,019 years observation time and 613 AGW diagnoses (incidence: 1.87/1,000 person-years). At the end of follow-up, $61 \%$ were $2 \mathrm{vHPV}$ vaccinated ( $\geq 1$ dose) of whom $91 \%$ were fully vaccinated. The AGW incidence was lower among those with $\geq 1$ dose versus 0 doses (alRR $0.75,95 \%$ confidence interval [CI] 0.64-0.88). The effect of vaccination was stronger after full vaccination (aIRR $0.72,95 \% \mathrm{Cl}$ $0.61-0.86$ ) and for women who were offered vaccination at $12-13$ years of age (aIRR 0.69 , $95 \% \mathrm{Cl} 0.51-0.93$ ) versus those at $13-16$ years of age (aIRR $0.77,95 \% \mathrm{Cl} 0.64-0.93$ ).

Discussion: This is the largest population-based study so far to examine the effect of 2vHPV vaccination on $A G W s$, with reliable individual information on $A G W$ diagnoses and vaccination status. The results indicate that $2 \mathrm{VHPV}$ vaccination partially protects against AGWs, especially when administered in early adolescence. 


\section{Introduction}

Human papillomavirus (HPV) is a highly contagious sexually transmitted virus and associated with the development of various cancers, recurrent respiratory papillomatosis, and anogenital warts (AGWs) [1-3]. Although AGWs are not life threatening, the burden and associated treatment costs are substantial [4]. In industrialized countries, the annual AGW incidence is $0.1 \%-0.2 \%$, with a peak occurring in women younger than 24 years of age [5]. Women with AGWs can experience mild symptoms such as pain, itch, and discharge from urethra or vagina. Moreover, AGWs can have a negative impact on the psychosocial wellbeing $[6,7]$. Treatment of AGWs is focused on the removal of visible warts and not on elimination of the underlying HPV infection, leading to high recurrence rates (up to $80 \%$ ) [8].

HPV types 6 and 11 are believed to cause the vast majority of the AGWs [2]. Currently 2 prophylactic vaccines are available that target HPV6/11 to prevent AGWs; the quadrivalent (4vHPV) vaccine and since 2015, the nonavalent (9vHPV) vaccine. Countries that have implemented these vaccines in their national immunization program (NIP) with high vaccination coverage have observed drastic declines in AGWs [9].

In the NIP of the Netherlands, the bivalent HPV (2vHPV) vaccine is used, which targets HPV16/18. The program started in 2009 with a one-off catch-up campaign for girls born in 1993-1996. Routine HPV vaccination started in 2010, offering vaccination to girls in the year they turn 13 years old, beginning with birth cohort 1997. Initially, vaccination was offered as a 3 -dose schedule $(0,1$, and 6 months), but in 2014 the program changed to a 2-dose schedule (0 and 6 months) [10].

Even though the 2vHPV vaccine does not target HPV6/11, there is ongoing debate about the possible protective effect of $2 \mathrm{VHPV}$ vaccination against AGWs. After introduction of 2 vHPV vaccination in the United Kingdom, a small but prominent decrease in AGW diagnoses was observed among young women at genitourinary medicine clinics, while such a decline was not observed for other sexually transmitted infections (STIs). The authors concluded that at least part of this decline was likely due to $2 \mathrm{vHPV}$ vaccination, and they calculated a vaccine effectiveness (VE) of 34\% [11]. Other studies from the United Kingdom, Czech Republic, and Spain did not find evidence for an effect of $2 \mathrm{vHPV}$ vaccination on AGWs [12-14]. In a previous study among visitors of sexual health centers in the Netherlands, we found a lower AGW prevalence among self-reported vaccinated compared with selfreported unvaccinated women, albeit statistically non-significant [15]. This study had low numbers of AGWs, partly because $95 \%$ of all AGW diagnoses in the Netherlands are made by general practitioners (GPs) [16].

Knowledge about the protective effect of $2 \mathrm{VHPV}$ vaccination against AGWs is important for comprehensive cost-effectiveness analyses and evidence-based communication 
strategies. Because the Netherlands is one of the few industrialized countries that have consistently used the 2VHPV vaccine in the NIP, we had the unique opportunity to study this effect in the general population. By linking patient data from GPs with data from the national immunization registry, we performed a retrospective registry-based open cohort study to assess the effect of 2vHPV vaccination on the incidence of AGWs diagnosed by GPs in the Netherlands.

\section{Methods}

\section{Study design and population}

The study population was constructed retrospectively by linking individual data from 3 data sources: (1) the Nivel Primary Care Database (Nivel-PCD) of GPs; (2) the national immunization registry (Præventis); and (3) the Dutch population registry (Supplementary Table 9.1). The study population included women born between 1993 and 2002 who were registered in a general practice (i.e. primary care) participating in the Nivel-PCD at some point during 2007-2015 and who were invited for HPV vaccination through the NIP. This was an open cohort, in which women could leave a general practice and re-enter later.

\section{Data sources}

Nivel-PCD

The Nivel-PCD provides a representative sample of general practices with respect to geographical distribution and urbanization degree, and a representative sample of about $10 \%$ of the Dutch population with respect to age and sex [17]. In the Netherlands, in principle, all inhabitants register in a general practice to have access to the health care system, irrespective of having healthcare consultations. GPs participating in the Nivel-PCD provide routinely recorded data from electronic medical files, including registration of patients, and data on diagnoses (using ICPC-1 codes) and prescriptions (using ATC codes). We restricted our analysis to data from general practices with complete ( $\geq 46$ weeks data per year) and good-quality data ( $\geq 70 \%$ of records with valid codes).

The period a woman was registered in a general practice, based on quarterly claim data, was used as the time-at-risk for AGW diagnoses. If a gap in registration was half a year or longer, we assumed that the patient left the general practice temporarily and this unregistered period was not included in the analyses. Gaps in the registration period of 1 quarter were ignored (Supplementary Figure 9.1).

We obtained data of consultations with the diagnoses AGWs (ICPC-1 code X91). As a proxy for sexual risk behavior, we used a consultation coded as fear of STIs (ICPC-1 code X23) or as fear of HIV/AIDS (ICPC-1 code B25). The mean number of GP consultations per year during follow-up was used as a proxy for health-seeking behavior. Finally, we collected 
data on consultations with the prescription 4VHPV vaccine (ATC code J07BM01) or 2vHPV vaccine (ATC code J07BM02).

\section{Præventis}

Præventis is the electronic national immunization registry including all vaccinations administered as part of the NIP [18]. We obtained individual data on whether someone was invited for HPV vaccination, the number and date of received HPV vaccine doses, and the type of vaccine given (2vHPV, 4vHPV, or unknown).

\section{Dutch population registry}

From the national population registry, we obtained data on the month and year of birth, migration background, and education level. The day of birth was set to 15 for all women. Migration background was based on (parental) country of birth [19]. Education level was based on the highest registered education a woman completed or was following as of December 2015. We categorized the education level as high (school of higher general secondary education, pre-university education, university of applied sciences, and university) or middle/low (all other forms of education).

The 3 data sources were linked using a unique identification number allocated by a trusted third party (Statistics Netherlands), based on either the encrypted citizen service number or a combination of sex, date of birth, and 4 digits of the postal code. Data extraction from Nivel-PCD has been approved according to the Nivel-PCD governance code under number NZR-00316.016. The study was exempt from formal medical-ethical approval under prevailing laws in the Netherlands as it concerned a retrospective observational study using de-identified data only. All parties granted approval for the usage and linkage of the data.

\section{Statistical analyses}

Women entered the cohort on 1 January 2007, on the date they were first registered in the GP's database, or on their 12th birthday, whichever came last. We excluded women who were HPV vaccinated in a general practice (outside the NIP), could not be matched to the other databases, or received 4VHPV or an unknown HPV vaccine as registered in Præventis. Women were followed until the first of the following: end of study period (31 December 2015); first AGW diagnosis registered during the follow-up period; or date of leaving the GP's database (end of follow-up). Only the first AGW diagnosis was included, because of the inability in this registry-based study to differentiate additional consultations as recurrent AGW diagnoses or as repeated consultations for the same wart. Observation time and events following the first AGW diagnoses were censored.

We calculated the adjusted incidence rate ratio (aIRR) of AGWs after being HPV vaccinated ( $\geq 1$ dose) relative to being unvaccinated, using Poisson regression with a log link 
function, log observation time as offset, and robust standard errors. Moreover, we made a distinction between being fully vaccinated ( 3 doses or 2 doses $\geq 5$ months apart, regardless of year of vaccination) or partially vaccinated ( 1 dose or 2 doses $<5$ months apart). Vaccination status was a time-dependent exposure, meaning that a woman could contribute observation time to the unvaccinated and the vaccinated category if she received vaccination during follow-up (Supplementary Figure 9.2). All analyses were adjusted for age as a time dependent variable. In addition, we adjusted for migration background, education level, having had a fear of STI/HIV consultation, and mean number of GP consultations per year. We also performed the analyses separately for birth cohorts 1996-2002 (cohorts who were offered vaccination according to the routine program at 12-13 years of age) and birth cohorts 1993-1995 (cohorts who were 13-16 years old when vaccination was offered).

The incubation period for AGWs is estimated to be between 3 and 18 months with a median of 6-10 months [4]. Because HPV vaccination has no effect on HPV types present at the time of vaccination [20], in sensitivity analyses we added buffer periods of 3,6 , and 10 months to the vaccination date to account for prevalent infections at the time of vaccination (Supplementary Figure 9.3). We also repeated the analyses excluding women with gaps in observation time.

The statistical analyses were performed using RStudio version 1.1.463 (RStudio, Boston, Massachusetts) with a statistical significance level of $p<.05$. Records with missing data were excluded from the analyses, as they represented less than $5 \%$ of the study population.

\section{Results}

\section{Study population}

In total, 131,379 women born between 1993 and 2002 were registered in a general practice participating in the Nivel-PCD between 2007 and 2015, of whom 129,125 (98\%) were registered in a practice with complete and good-quality data. After applying all exclusion criteria, 96,468 women (73\%) were included in the final cohort. The majority of the women who were excluded could not be matched to the other databases; a limited number of women were excluded because they were vaccinated by the GP $(n=124)$ or with the 4vHPV or unknown HPV vaccine ( $n=249$ ) (Figure 9.1). Together, the women contributed 328,019 years of observation time (median: 3 years per woman). Table 9.1 presents the characteristics of the study population.

In total, 28,865 women (29.9\%) were already vaccinated at least once when entering the cohort and 30,609 women (31.7\%) were vaccinated with at least 1 dose during followup. At the end of follow-up 58,793 women (60.9\%) were vaccinated with the 2 vHPV vaccine ( $\geq 1$ dose); 53,744 (55.7\%) were fully vaccinated, and 5,049 (5.2\%) were partially vaccinated. 


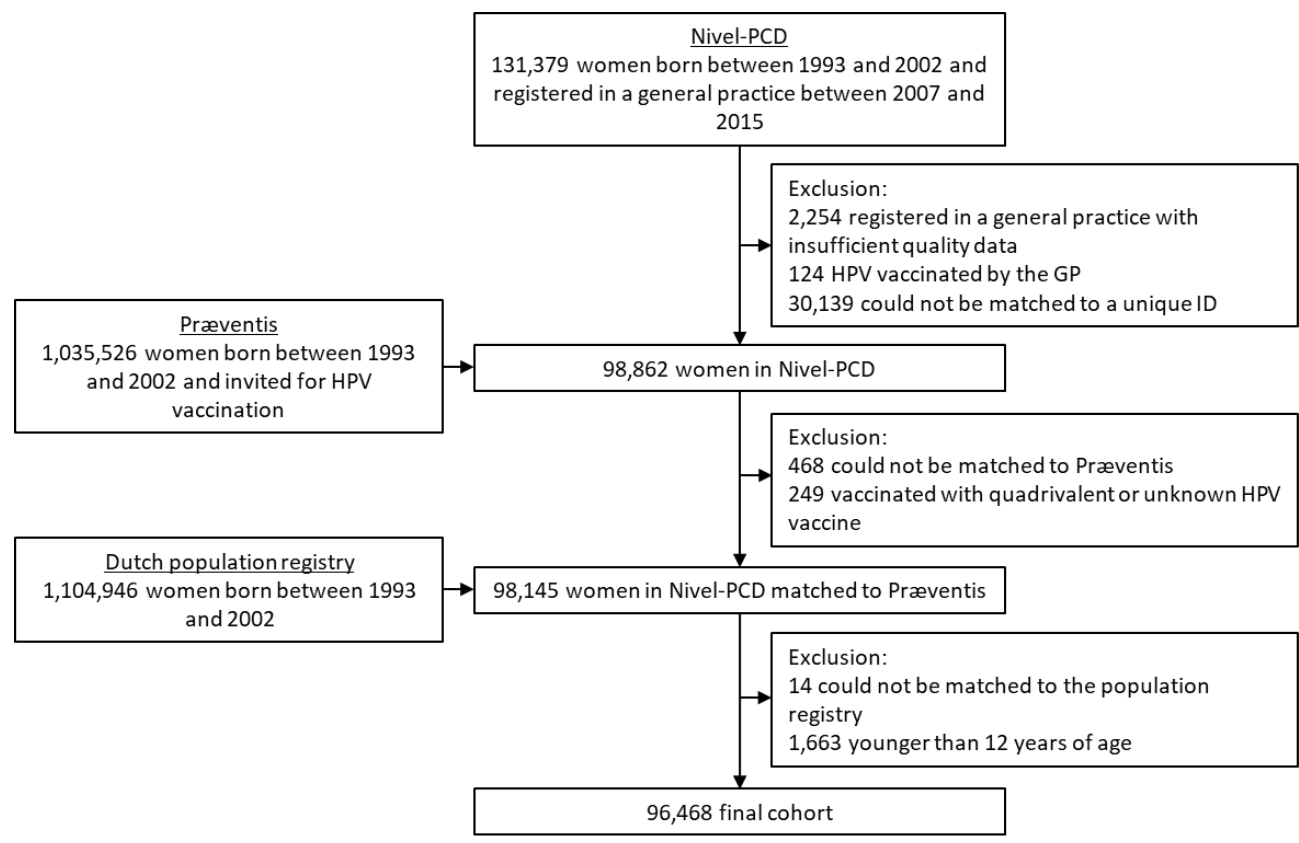

Figure 9.1. Study flowchart.

Note: ID: Identifier; Nivel-PCD: network of representative general practice across the Netherlands; Præventis: national immunization registry.

Table 9.1. Characteristics of the study population included in the analyses.

Total study population $(\mathrm{N}=96,468)$

n

$\%$

\begin{tabular}{|c|c|c|}
\hline \multicolumn{3}{|l|}{ Observation time in years per womana } \\
\hline Median $\left(25^{\text {th }}-75^{\text {th }}\right.$ percentile $)$ & \multicolumn{2}{|c|}{$3.0(2.0-5.0)$} \\
\hline \multicolumn{3}{|l|}{ Vaccination status at the start of follow-up } \\
\hline Unvaccinated & 67,603 & 70.1 \\
\hline Partially vaccinated ${ }^{b}$ & 2,783 & 2.9 \\
\hline Fully vaccinated ${ }^{b}$ & 26,082 & 27.0 \\
\hline \multicolumn{3}{|l|}{ Vaccination status at the end of follow-up } \\
\hline Unvaccinated & 37,675 & 39.1 \\
\hline Partially vaccinated ${ }^{b}$ & 5,049 & 5.2 \\
\hline Fully vaccinated ${ }^{b}$ & 53,744 & 55.7 \\
\hline \multicolumn{3}{|l|}{ Age (years) } \\
\hline Range & $12.00-$ & \\
\hline Median $\left(25^{\text {th }}-75^{\text {th }}\right.$ percentile) at the start of follow-up & $13.4(12$ & \\
\hline Median $\left(25^{\text {th }}-75^{\text {th }}\right.$ percentile $)$ at the end of follow-up & $17.7(15$ & \\
\hline
\end{tabular}


Table 9.1. (Continued).

\begin{tabular}{|c|c|c|}
\hline & \multicolumn{2}{|c|}{ Total study population $(\mathrm{N}=96,468)$} \\
\hline & $\mathrm{n}$ & $\%$ \\
\hline \multicolumn{3}{|l|}{ Migration background $^{c}$} \\
\hline Native Dutch & 75,595 & 78.4 \\
\hline Moroccan & 2,994 & 3.1 \\
\hline Turkish & 2,981 & 3.1 \\
\hline Surinamese & 2,249 & 2.3 \\
\hline Antillean/Aruban & 1,139 & 1.2 \\
\hline Western (non-Dutch) & 6,158 & 6.4 \\
\hline Non-Western (other than above) & 5,352 & 5.5 \\
\hline \multicolumn{3}{|l|}{ Education level $^{d}$} \\
\hline Low/middle & 50,537 & 52.4 \\
\hline High & 44,563 & 46.2 \\
\hline Missing & 1,368 & 1.4 \\
\hline \multicolumn{3}{|c|}{ Fear of STI or fear of HIV/AIDS consultatione } \\
\hline No & 92,753 & 96.1 \\
\hline Yes & 3,715 & 3.9 \\
\hline \multicolumn{3}{|c|}{ Mean number of GP consultations per year } \\
\hline Median $\left(25^{\text {th }}-75^{\text {th }}\right.$ percentile $)$ & $2.2(0-$ & \\
\hline \multicolumn{3}{|c|}{$\begin{array}{l}\text { a. Observation time was defined as the period a women was registered in a general practice. Follow-up started the first time a } \\
\text { woman was registered in a general practice from } 1 \text { January } 2007 \text { onwards or on the } 12 \text { th birthday. Follow-up ended when a } \\
\text { women was diagnosed with AGWs, left the general practice, or end of study period ( } 31 \text { December 2015). } \\
\text { b. Partially vaccinated: } 1 \text { dose or } 2 \text { doses }<5 \text { months apart. Fully vaccinated: } 3 \text { doses or } 2 \text { doses } \geq 5 \text { months apart. } \\
\text { c. Migration background was based on parental country of birth. } \\
\text { d. High educational level included school of higher general secondary education, pre-university education, university of applied } \\
\text { sciences, and university. Low/middle educational level included all other levels of education. } \\
\text { e. ICPC-1 code X23 or B25 (fear of STI or fear of HIV/AIDS) prior to an AGW diagnoses. }\end{array}$} \\
\hline
\end{tabular}

\section{Anogenital warts}

In total, 613 women had an AGW diagnosis registered by a GP during the follow-up period (overall incidence 1.87/1,000 person-years). The AGW incidence increased steadily with age, with a peak at 21 years of age (7.21/1,000 person-years). The incidence of AGWs was lower for vaccinated compared to unvaccinated women across all ages (Figure 9.2).

The AGW incidence was lower after $\geq 1$ dose $2 \mathrm{VHPV}$ vaccine relative to 0 doses (aIRR $0.75,95 \%$ confidence interval $[\mathrm{Cl}]$ 0.64-0.88) (Table 9.2). The effect was stronger after being fully vaccinated (aIRR $0.72,95 \% \mathrm{Cl} 0.61-0.86$ ) and not observed after being partially vaccinated (aIRR $0.96,95 \% \mathrm{Cl} 0.68-1.32$ ), although the difference between fully and partially vaccinated itself was not statistically significant $(p=0.1)$.

Overall, 67,179 women (70\%) were born between 1996 and 2002 (12-13 years of age when vaccination was offered) and 29,289 (30\%) were born between 1993 and 1995 (13-16 years of age when vaccination was offered). The aIRR of $\geq 1$ dose relative to 0 doses was 
0.69 (95\% $\mathrm{Cl} 0.51-0.93$ ) for the younger birth cohorts and 0.77 (95\% $\mathrm{Cl} 0.64-0.93$ ) for the older birth cohorts. After a full vaccination course these figures were $0.60(95 \% \mathrm{Cl} 0.44-$ 0.83 ) and 0.77 (95\% $\mathrm{Cl} 0.63-0.93)$ respectively (Table 9.3).

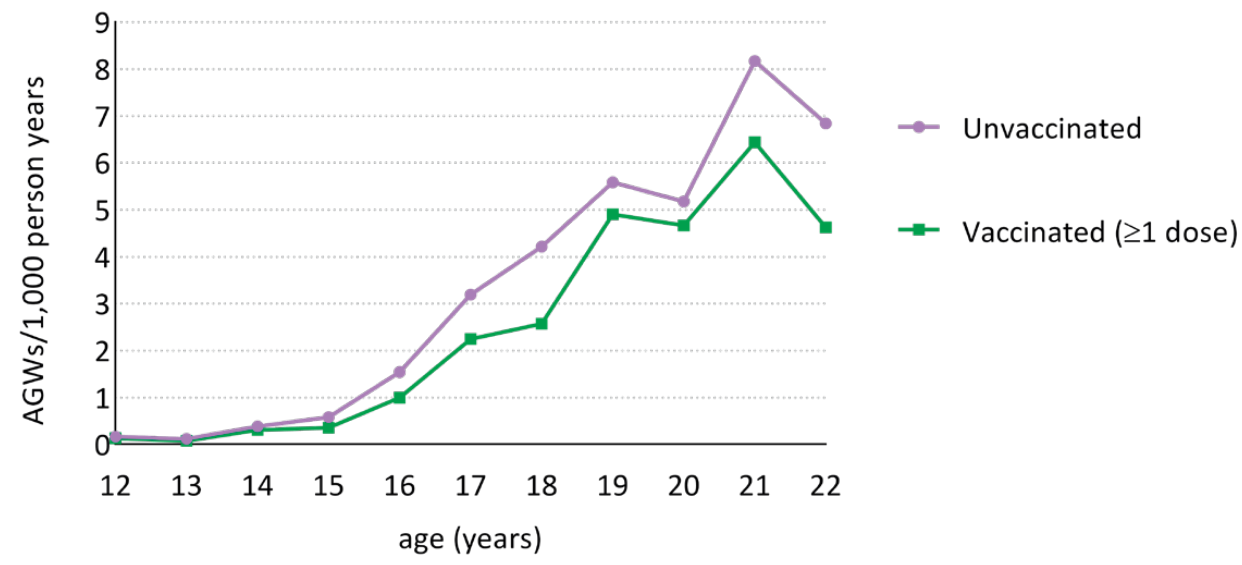

Figure 9.2. Incidence of AGWs by age and vaccination status.

Table 9.2. Association between bivalent HPV vaccination and AGWs diagnosed by GPs.

\begin{tabular}{llllll}
\hline & $\mathbf{N}^{\mathbf{a}}$ & $\begin{array}{l}\text { Observation } \\
\text { time, } \mathbf{y}\end{array}$ & $\begin{array}{l}\text { AGW } \\
\text { diagnoses }\end{array}$ & alRR $^{\mathbf{b}} \mathbf{( 9 5 \% \mathbf { C l } )}$ & alRR $\left.^{\mathbf{c}} \mathbf{( 9 5 \%} \mathbf{C l}\right)$ \\
\hline $\begin{array}{l}\text { Vaccination status } \\
\text { Unvaccinated }\end{array}$ & 66,487 & 144,129 & 296 & Reference & Reference \\
$\begin{array}{l}\text { Vaccinated ( } \geq 1 \text { dose) } \\
\text { Vaccination status }\end{array}$ & 58,299 & 180,497 & 310 & $0.76(0.65-0.89)$ & $0.75(0.64-0.88)$ \\
$\begin{array}{l}\text { Unvaccinated } \\
\text { Partially vaccinated }\end{array}$ & 66,487 & 144,129 & 296 & Reference & Reference \\
Fully vaccinated $^{d}$ & 31,790 & 26,409 & 42 & $1.15(0.82-1.57)$ & $0.96(0.68-1.32)$ \\
\hline
\end{tabular}

a. Number of women that contributed observation time per vaccination status. One woman could contribute observation time to $>1$ vaccination status. Women with missing education level were excluded.

b. Adjusted for age as time-varying.

c. Adjusted for age as time-varying, migration background, education level, fear of STI/HIV consultations, and mean number of GP consultations per year.

d. Partially vaccinated: 1 dose or 2 doses $<5$ months apart. Fully vaccinated: 3 doses or 2 doses $\geq 5$ months apart.

\section{Sensitivity analyses}

In both sensitivity analyses, results were not meaningfully different from the main analyses (Supplementary Table 9.2). With a buffer period of 10 months, the effect of vaccination was slightly smaller (aIRR $\geq 1$ dose 0.77 compared to 0.75 in the main analyses), whereas the effect was slightly larger (aIRR $\geq 1$ dose 0.70 ) when only women without gaps in observation time $(n=87,612 ; 91 \%)$ were included. 
Table 9.3. Association between bivalent HPV vaccination and AGWs diagnosed by GPs, stratified by birth cohorts.

\begin{tabular}{|c|c|c|c|c|c|}
\hline & $\mathbf{N}^{\mathrm{a}}$ & $\begin{array}{l}\text { Observation } \\
\text { time, y }\end{array}$ & $\begin{array}{l}\text { AGW } \\
\text { diagnoses }\end{array}$ & $\operatorname{aIRR}^{b}(95 \% \mathrm{Cl})$ & $\operatorname{alRR}^{c}(95 \% \mathrm{Cl})$ \\
\hline \multicolumn{6}{|c|}{ Cohorts 1996-2002d } \\
\hline \multicolumn{6}{|l|}{ Vaccination status } \\
\hline Unvaccinated & 51,454 & 91,815 & 85 & Reference & Reference \\
\hline Vaccinated ( $\geq 1$ dose) & 41,921 & 116,874 & 93 & $0.68(0.51-0.91)$ & $0.69(0.51-0.93)$ \\
\hline \multicolumn{6}{|l|}{ Vaccination status } \\
\hline Unvaccinated & 51,454 & 91,815 & 85 & Reference & Reference \\
\hline Partially vaccinated & 28,022 & 20,219 & 20 & 1.99 (1.18 - 3.19) & $1.47(0.86-2.40)$ \\
\hline Fully vaccinated ${ }^{e}$ & 38,381 & 96,655 & 73 & $0.58(0.42-0.79)$ & $0.60(0.44-0.83)$ \\
\hline \multicolumn{6}{|c|}{ Cohorts 1993-1995d } \\
\hline \multicolumn{6}{|l|}{ Vaccination status } \\
\hline Unvaccinated & 15,033 & 52,314 & 211 & Reference & Reference \\
\hline Vaccinated ( $\geq 1$ dose) & 16,378 & 63,623 & 217 & $0.79(0.65-0.95)$ & $0.77(0.64-0.93)$ \\
\hline \multicolumn{6}{|l|}{ Vaccination status } \\
\hline Unvaccinated & 15,033 & 52,314 & 211 & Reference & Reference \\
\hline Partially vaccinatede & 3,768 & 6,190 & 22 & $0.92(0.57-1.39)$ & $0.80(0.50-1.22)$ \\
\hline Fully vaccinatede & 15,008 & 57,433 & 195 & $0.78(0.64-0.94)$ & $0.77(0.63-0.93)$ \\
\hline \multicolumn{6}{|c|}{$\begin{array}{l}\text { a. Number of women that contributed observation time per vaccination status. One woman could contribute observation time } \\
\text { to }>1 \text { vaccination status. Women with missing education level were excluded. } \\
\text { b. Adjusted for age as time-varying. } \\
\text { c. Adjusted for age as time-varying, migration background, education level, fear of STI/HIV consultations, and mean number of } \\
\text { GP consultations per year. } \\
\text { d. Birth cohorts } 1996-2002 \text { were } 12-13 \text { years of age when vaccination was offered. Birth cohorts 1993-1995 were 13-16 years } \\
\text { of age when vaccination was offered. }\end{array}$} \\
\hline
\end{tabular}

\section{Discussion}

We studied the effect of $2 \mathrm{vHPV}$ vaccination on AGWs diagnosed by GPs in the Netherlands by linking GP data to the vaccination registry. We showed a significantly reduced incidence of AGWs among 2vHPV vaccinated women compared to unvaccinated women. The effect of vaccination was stronger after completion of the vaccination course and for women offered vaccination at 12-13 years of age.

To our knowledge, this is the largest study so far to assess the direct effect of 2vHPV vaccination on clinically relevant AGWs. To minimize bias we used population-based data with reliable vaccination status and AGWs diagnosed at the general practice, where about 95\% of all AGW diagnoses in the Netherlands are made [16]. We do acknowledge some limitations. First, not all women in the GP's database could be linked to the vaccination registry. Comparing our study population to all women born between 1993 and 2002 living in the Netherlands, our study population was slightly more often native Dutch (78\% vs $71 \%$ ), 
highly educated ( $46 \%$ vs $43 \%$ ), and HPV vaccinated (62\% vs $58 \%$ as of December 2015 ). This suggests that our study population might not have been fully representative for the total Dutch population. Because a native Dutch background and high educational level were associated with a lower risk for AGWs, we might have underestimated the overall AGW incidence, but this effect is likely to be small. Second, women were defined to enter or leave the cohort depending on registration in a general practice. Therefore, women might have already been diagnosed with AGWs before entering the cohort. Assuming non-differential misclassification between vaccinated and unvaccinated women, this would lead to an underestimation of the protective effect of vaccination. Misclassification of vaccination status is unlikely, because virtually all vaccinations are registered in Præventis (as demonstrated in our study: only $0.1 \%$ was vaccinated by the GP).

The preferred design to measure a causal direct effect of vaccination would be a randomized placebo-controlled trial (RCT), but RCTs of the 2vHPV vaccine did not include AGWs among reported outcomes [21]. Performing an RCT now would be unethical, because of the already demonstrated very high efficacy against oncogenic HPV types and associated precancer lesions [22]. Investigation into the effect of 2vHPV vaccination on AGWs therefore relies on large observational studies with minimal risk of bias. However, we cannot rule out residual confounding, implying that the lower observed incidence of AGWs among 2 VHPV vaccinated women could be related to an overall lower risk of AGWs among vaccinated compared to unvaccinated women, unrelated to vaccination itself. We had limited information on possible confounders to fully adjust for this. Previous studies did observe small differences in risk behavior between HPV vaccinated and unvaccinated women in the Netherlands, but these variables hardly affected the association between vaccination and HPV outcome measures, suggesting limited confounding [23].

An explanation for the observed lower incidence of AGWs among 2vHPV vaccinated women is that $2 \mathrm{vHPV}$ vaccination also effectively protects against AGWs. This supposition is supported by a number of observations. First, we observed a dose-response relation, with stronger effects after a complete vaccination course compared to an incomplete vaccination course. Similar results were observed elsewhere for the 4vHPV vaccine against AGWs [24]. Second, the effect of vaccination was lower for the older birth cohorts in our analysis. This is in line with the observed lower VE among women with HPV exposure prior to vaccination, which is more likely if vaccination is offered at an older age [22]. Last, our results were very robust, with an effect of $2 \mathrm{VHPV}$ vaccination that was consistent across all ages and not affected by considerations of a buffer period or complete observation time.

The biological mechanism behind the apparent effectiveness of $2 \mathrm{vHPV}$ vaccination against AGWs is not clear. One possible explanation is that high-risk HPV (hrHPV) types play a larger role in the development of AGWs than generally assumed [25]. HrHPV types, 
including the 2VHPV vaccine types HPV16/18, are frequently genotyped in AGWs (generally ranging between $30 \%$ and $40 \%$; in some AGWs only hrHPV types are genotyped) [2, 26-29]. Because the 2vHPV vaccine induces excellent effectiveness against HPV16/18 and considerable cross-protection against several other hrHPV types [30-32], a putative causative contribution of hrHPV types to the development of AGWs would also lead to a partial protective effect of $2 \mathrm{vHPV}$ vaccination against AGWs. Another possible explanation is that 2vHPV vaccination provides cross-protection against HPV6/11, which was observed in the largest RCT of the 2vHPV vaccine [21]. However, only 1 of the post-marketing surveillance studies replicated this effect [33] and most could not [15, 23, 34]. Moreover, cross-protection against HPV6/11 seems unlikely based on the large phylogenetic distance between these low-risk types and the vaccine types HPV16/18 [32]. However, it might be that $2 \mathrm{vHPV}$ vaccination could prevent development of AGWs by HPV6/11 infections, as suggested by significantly reduced HPV6 viral loads among 2vHPV vaccinated relative to unvaccinated women in the Netherlands [35]. The RCT of the 2vHPV vaccine also showed that vaccination might be more effective against clinical outcomes than against HPV infections itself; the protection against cervical intraepithelial neoplasia grade 3 was higher than what was expected based on the protection against type-specific HPV infections [22]. This effect could be related to T-cell responses, triggered by the ASO4 adjuvant of the 2vHPV vaccine, that are presumably broadly cross-protective [36-38].

Our results correspond to a relevant VE of $23 \%-40 \%$, depending on completion of the vaccination course and age at vaccination. A protective effect against AGWs further contributes to the already very attractive cost-effectiveness profile of the $2 \mathrm{vHPV}$ vaccine [39]. This effect was not considered in previous health economic comparisons of the 2vHPV vaccine with the 4VHPV or 9vHPV vaccine [40]. Even so, protection against AGWs should be considered a beneficial side effect, as protection against hrHPV types remains the main objective in $2 \mathrm{vHPV}$ vaccination programs.

In conclusion, in this large population-based study using data from different registries, the incidence of AGWs was significantly reduced among $2 \mathrm{VHPV}$ vaccinated compared with unvaccinated women, suggestive of a partially protective effect of $2 \mathrm{vHPV}$ vaccination against AGWs. Our findings merit further investigation into the possible mechanisms behind the effect. This could give more insights into the natural history of AGWs and the protective effects of HPV vaccination. 


\section{References}

1. Chaturvedi AK. Beyond cervical cancer: burden of other HPV-related cancers among men and women. J Adolesc Health 2010; 46:S20-6.

2. Garland SM, et al. Natural history of genital warts: analysis of the placebo arm of 2 randomized phase III trials of a quadrivalent human papillomavirus (types $6,11,16$, and 18) vaccine. J Infect Dis 2009; 199:80514.

3. Carifi $M$, et al. Recurrent respiratory papillomatosis: current and future perspectives. Ther Clin Risk Manag 2015; 11:731-8.

4. Park IU, et al. Human Papillomavirus and Genital Warts: A Review of the Evidence for the 2015 Centers for Disease Control and Prevention Sexually Transmitted Diseases Treatment Guidelines. Clin Infect Dis 2015; 61 Suppl 8:S849-55.

5. Forman D, et al. Global burden of human papillomavirus and related diseases. Vaccine 2012; 30 Suppl 5:F12-23.

6. Vriend $\mathrm{HJ}$, et al. Impact of genital warts on emotional and sexual well-being differs by gender. Int J STD AIDS 2014; 25:949-55.

7. Dominiak-Felden G, et al. Impact of human papillomavirus-related genital diseases on quality of life and psychosocial wellbeing: results of an observational, health-related quality of life study in the UK. BMC Public Health 2013; 13:1065.

8. Patel $\mathrm{H}$, et al. Systematic review of the incidence and prevalence of genital warts. BMC Infect Dis 2013; 13:39.

9. Drolet $\mathrm{M}$, et al. Population-level impact and herd effects following the introduction of human papillomavirus vaccination programmes: updated systematic review and meta-analysis. Lancet 2019; 394:497-509.

10. Qendri V, et al. Ten years of HPV vaccination in the Netherlands: current evidence and future challenges in HPV-related disease prevention. Expert Rev Vaccines 2018; 17:1093-104.

11. Howell-Jones R, et al. Declining Genital Warts in Young Women in England Associated With HPV 16/18 Vaccination: An Ecological Study. J Infect Dis 2013; 208:1397-403.

12. Sonnenberg $P$, et al. Epidemiology of genital warts in the British population: implications for HPV vaccination programmes. Sex Transm Infect 2019; 95:386-90.

13. Petras $M$, et al. Impact of quadrivalent human papillomavirus vaccine in women at increased risk of genital warts burden: Population-based crosssectional survey of Czech women aged 16 to 40 years. Vaccine 2015; 33:6264-7.

14. Navarro-Illana E, et al. Effectiveness of HPV vaccines against genital warts in women from Valencia, Spain. Vaccine 2017; 35:3342-6.

15. Woestenberg PJ, et al. No evidence for crossprotection of the HPV-16/18 vaccine against HPV$6 / 11$ positivity in female STI clinic visitors. J Infect 2017; 74:393-400.

16. Van den Broek IVF, et al. Sexually tranmitted infections in the Netherlands in 2015. Bilthoven: National Institute for Public Health and the Environment, 2016. Nr: 2016-0027.

17. Boersma-van Dam ME, et al. Zorg door de huisarts. Uit: Zorg door de huisarts - Nivel Zorgregistraties Eerste Lijn: Jaarcijfers 2017 en trendcijfers 2011-2017. Verantwoording cijfers huisartsenzorg. Uit: Nivel Zorgregistraties eerste lijn. [Dutch]. Available at: www.nivel.nl/nl/nivelzorgregistraties-eerste-lijn/huisartsen. Accessed 2510-2019.

18. van Lier A, et al. Præventis, the immunisation register of the Netherlands: a tool to evaluate the National Immunisation Programme. Euro Surveill 2012; 17.

19. Woestenberg PJ, et al. Comparison of STI-related consultations among ethnic groups in the Netherlands: an epidemiologic study using electronic records from general practices. BMC Fam Pract 2015; 16:70.

20. Szarewski A, et al. Efficacy of the human papillomavirus (HPV)-16/18 AS04-adjuvanted vaccine in women aged $15-25$ years with and without serological evidence of previous exposure to HPV16/18. Int J Cancer 2012; 131:106-16.

21. Szarewski A, et al. Efficacy of the HPV-16/18 AS04-adjuvanted vaccine against low-risk HPV types (PATRICIA randomized trial): an unexpected observation. J Infect Dis 2013; 208:1391-6. 
22. Lehtinen $M$, et al. Overall efficacy of HPV-16/18 AS04-adjuvanted vaccine against grade 3 or greater cervical intraepithelial neoplasia: 4-year end-ofstudy analysis of the randomised, double-blind PATRICIA trial. Lancet Oncol 2012; 13:89-99.

23. Donken R, et al. High Effectiveness of the Bivalent Human Papillomavirus (HPV) Vaccine Against Incident and Persistent HPV Infections up to 6 Years After Vaccination in Young Dutch Women. J Infect Dis 2018; 217:1579-89.

24. Markowitz LE, et al. Human papillomavirus vaccine effectiveness by number of doses: Systematic review of data from national immunization programs. Vaccine 2018; 36:4806-15.

25. Hasanzadeh $M$, et al. The interaction of high and low-risk human papillomavirus genotypes increases the risk of developing genital warts: A populationbased cohort study. J Cell Biochem 2019; 120:128704.

26. Aubin $F$, et al. Human papillomavirus genotype distribution in external acuminata condylomata: a Large French National Study (EDiTH IV). Clin Infect Dis 2008; 47:610-5.

27. Sturegard E, et al. Human papillomavirus typing in reporting of condyloma. Sex Transm Dis 2013; 40:123-9.

28. Vandepapeliere $P$, et al. Randomized controlled trial of an adjuvanted human papillomavirus (HPV) type 6 L2E7 vaccine: infection of external anogenital warts with multiple HPV types and failure of therapeutic vaccination. J Infect Dis 2005; 192:2099107.

29. Zhu C, et al. Prevalence and distribution of HPV types in genital warts in Xi'an, China: a prospective study. BMJ Open 2019; 9:e023897.

30. Skinner SR, et al. Human papillomavirus (HPV)16/18 AS04-adjuvanted vaccine for the prevention of cervical cancer and HPV-related diseases. Expert Rev Vaccines 2016; 15:367-87.
31. Woestenberg PJ, et al. Bivalent Vaccine Effectiveness Against Type-Specific HPV Positivity: Evidence for Cross-Protection Against Oncogenic Types Among Dutch STI Clinic Visitors. J Infect Dis 2018; 217:213-22.

32. Bogaards JA, et al. Bivalent HPV Vaccine Effectiveness Correlates with Phylogenetic Distance from Hpv Vaccine Types 16 And 18. J Infect Dis 2019. 33. Latsuzbaia A, et al. Effectiveness of bivalent and quadrivalent human papillomavirus vaccination in Luxembourg. Cancer Epidemiol 2019; 63:101593.

34. Cameron RL, et al. Human Papillomavirus Prevalence and Herd Immunity after Introduction of Vaccination Program, Scotland, 2009-2013. Emerg Infect Dis 2016; 22:56-64.

35. van der Weele $P$, et al. Effect of the bivalent HPV vaccine on viral load of vaccine and non-vaccine HPV types in incident clearing and persistent infections in young Dutch females. PLoS One 2019; 14:e0212927. 36. Pinto $L A$, et al. Cellular immune responses to HPV-18, -31, and -53 in healthy volunteers immunized with recombinant HPV-16 L1 virus-like particles. Virology 2006; 353:451-62.

37. Evans TG, et al. A Phase 1 study of a recombinant viruslike particle vaccine against human papillomavirus type 11 in healthy adult volunteers. J Infect Dis 2001; 183:1485-93.

38. Del Giudice G, et al. Correlates of adjuvanticity: A review on adjuvants in licensed vaccines. Semin Immunol 2018; 39:14-21.

39. Qendri V, et al. Health and Economic Impact of a Tender-Based, Sex-Neutral Human Papillomavirus 16/18 Vaccination Program in the Netherlands. J Infect Dis 2017; 216:210-9.

40. Jit $M$, et al. Comparing bivalent and quadrivalent human papillomavirus vaccines: economic evaluation based on transmission model. BMJ 2011; 343:d5775. 


\section{Supplementary information to Chapter 9}

Supplementary Table 9.1. Overview of the 3 registries used for this study and the data that were gathered for the current research question from each registry.

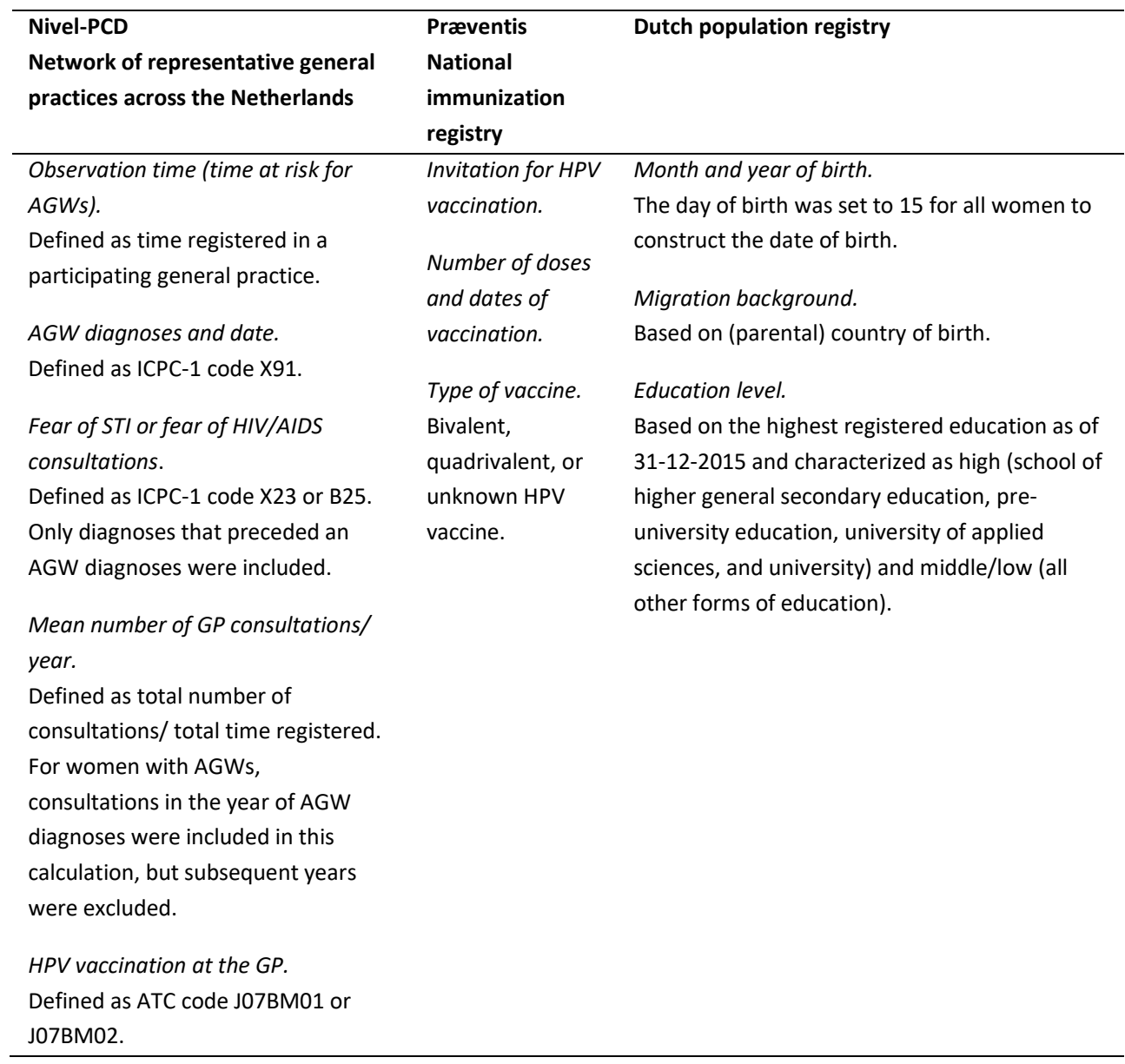


1

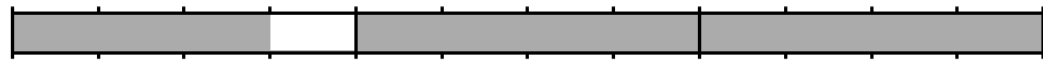

2

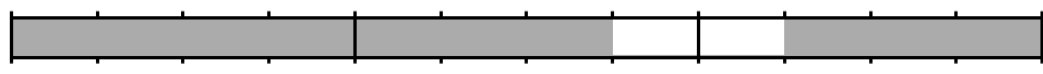

3

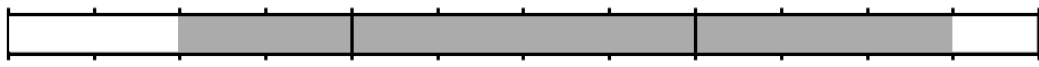

Quartiles

Calendar year

\begin{tabular}{|l|l|l|l|l|l|l|l|l|l|l|l|}
\hline 1 & 2 & 3 & 4 & 1 & 2 & 3 & 4 & 1 & 2 & 3 & 4 \\
\hline \multicolumn{3}{|c|}{1} \\
\hline
\end{tabular}

Observation time

Supplementary Figure 9.1. Examples of the quarterly registration in a general practice and the assumption regarding gaps in registration time.

This hypothetical cohort shows 3 examples of the registration in a general practice and the assumption regarding gaps is registration time.

1. Woman 1 was not registered in a general practice the last quartile of the first year. Because the gap in registration time was only 0.25 year, we ignored this gap and assumed the patient was also registered during this 0.25 -year period. Women 1 contributed 3 person-years of observation time.

2. Woman 2 was not registered in a general practice the last quartile of the second year and the first quartile of the third year. Because the gap in registration time was 0.5 year, this period was not included in the analyses. Women 2 contributed 2.5 person-years of observation time.

3. Women 3 was not registered the first half of year 1 and the last quartile of year 3 . Because the unregistered periods of women 3 were at the beginning and at the end of follow-up, these were not "gaps" in registration time and not included in the analyses. Women 3 contributed 2.25 person-year of observation time. 
1

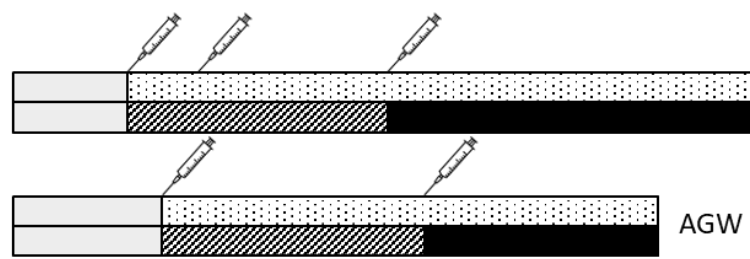

3

4

AGW

5

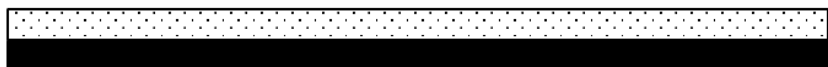

6

7

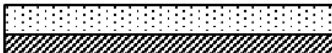

7

Observation time unvaccinated

Observation time vaccinated $(\geq 1$ dose $) \longrightarrow$ Observation time fully vaccinated

Supplementary Figure 9.2. Examples of the inclusion of vaccination status as a time-dependent variable.

Women entered the cohort the on 1 January, 2007 (women 1, 2, 3, 4 in this hypothetical cohort) or any time after that (women 5, 6, 7). Women were followed until the: first AGW diagnosis (women 2, 4); date of leaving the general practice (women 6, 7); or end of the study period (women 1, 3, 5). Vaccination status was time-dependent; a woman could contribute observation time to the different vaccination status categories depending on the number of doses she received during follow-up. For example women 1 and 2 were vaccinated during follow-up and contributed observation time to the unvaccinated as well as vaccinated status. Women 5 and 7 were already vaccinated when entering the cohort and only contributed observation time to the vaccinated status.

Note: Partially vaccinated included 1 dose or 2 doses $<5$ months apart. Fully vaccinated included 3 doses or 2 doses $\geq 5$ months apart. 
1

No BP

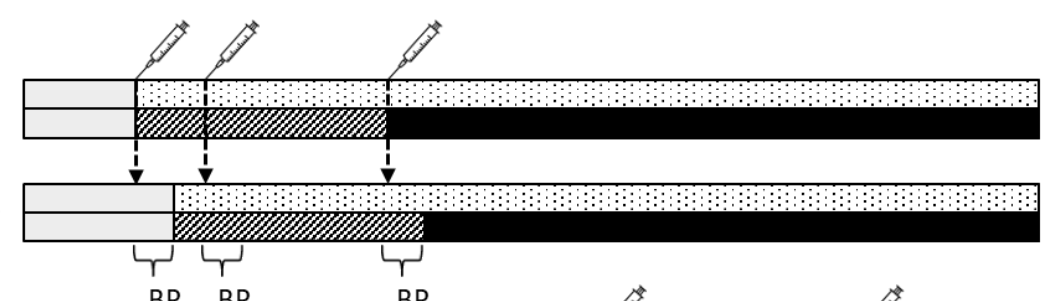

$3 m$ BP

BP BP

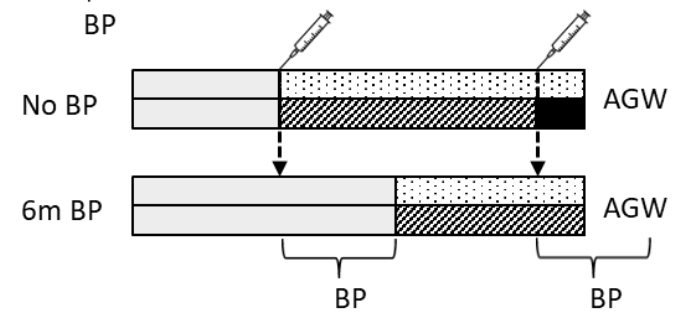

Observation time unvaccinated

Observation time partially vaccinated

Observation time fully vaccinated

Supplementary Figure 9.3. Examples of the inclusion of a buffer period.

A buffer period (BP) was added to the dates of vaccine doses in sensitivity analyses. This hypothetical cohort shows 2 examples, 1 including a 3-month BP and 1 including a 6-month BP. An unvaccinated woman contributes to unvaccinated observation time until she receives her first vaccine dose plus the BP. Then, she starts contributing to vaccinated observation time. Woman 1 contributes person-time to incomplete dosing until she received her third vaccine dose plus the BP. Due to the inclusion of a $B P$, the AGW diagnoses of woman 2 was attributed to the partially vaccinated observation time instead of full vaccination observation time.

Note: Partially vaccinated included 1 dose or 2 doses $<5$ months apart. Fully vaccinated included 3 doses or 2 doses $\geq 5$ months apart. 


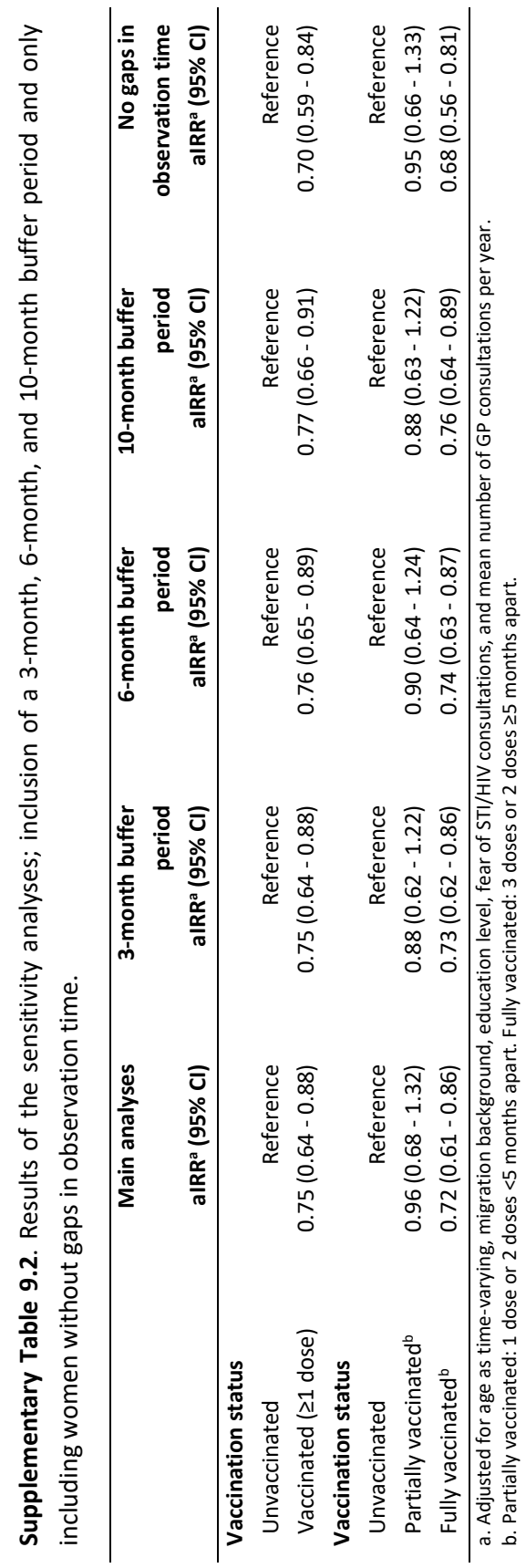





\section{Chapter 10}

General discussion 
The aim of the studies described in this thesis was to measure the individual- and population-level effects of HPV16/18 vaccination in the Netherlands. We measured the direct vaccine effectiveness (VE) of bivalent HPV (2vHPV) vaccination against HPV positivity and anogenital warts (AGWs) and the impact of the HPV vaccination program on the HPV prevalence in a high-risk population. For most of the studies included in this thesis, we used data of the PASSYON study where the HPV prevalence was repeatedly tested among visitors of sexual health centers (SHCs) throughout the Netherlands. In the PASSYON study, data was collected on the HPV prevalence in the pre- and post-vaccination period and on selfreported vaccination status, allowing to differentiate between distinct vaccine effects; direct effects (VE), indirect effects, and the overall impact of the vaccination program (Figure 1.3).

The HPV vaccination program in the Netherlands has already changed several times since its introduction in 2009; it started as a multi-cohort catch-up campaign for 12- to 16year-old girls, before settling on routine vaccination of girls in the year they turn 13 years old since 2010. In addition, in 2014 the 3-dose vaccination schedule was changed to a 2dose schedule [1]. And more changes in the HPV vaccination program are to come. In 2019, the Dutch Health Council advised to extent vaccination to boys (sex-neutral vaccination). The Health Council also advised to change the start of the vaccination from 12/13 years of age to $9 / 10$ years of age and to provide free of charge catch-up vaccination to everyone up to 26 years of age [2]. These recommendations were positively received by the Ministry of Health, Welfare, and Sports (VWS), and are planned to be implemented in the Netherlands in 2021 [3]. Monitoring the effects of this new vaccination program will also be of importance and can be informed by insights obtained in this thesis.

In the next sections, we will summarize and discuss the main findings of this thesis. Moreover, we will discuss future perspectives for measuring the effects of HPV vaccination, keeping in mind the imminent changes in the HPV vaccination program.

\section{Summary and discussion of main findings}

\section{Effects on HPV positivity}

In Part 1 of this thesis, we measured the VE against HPV positivity using data from the PASSYON study by comparing the HPV prevalence between self-reported vaccinated and unvaccinated women. An assumption in calculating the $V E$, is equal susceptibility and exposure to the infection, which was violated by differences in demographics and risk behavior between vaccinated and unvaccinated women. To provide the best approximation of the VE, the available information on risk factors for HPV infections were used to adjust for differences by vaccination status. The dependence of multiple HPV types within an 
individual was also taken into consideration (mixed effects). Despite these corrections however, there is always a risk of residual confounding in observational studies. In Chapter 2, we estimated a high VE against genital HPV16 and HPV18 positivity; the 2VHPV vaccine types. In line with prophylactic vaccination, we observed a higher VE among women who were not yet sexually active when vaccination was offered. Moreover, we observed a lower, but statistically significant VE against non-vaccine high-risk HPV (hrHPV) types 31, 35, 45, and 52. In Chapter 3, we updated the analyses with more data and observed that the level of cross-protection against non-vaccine types was highly correlated with the phylogenetic distance of these types towards the vaccine types HPV16/18. Based on these analyses, 2VHPV vaccination was predicted to provide partial VE against HPV types 31, 33, 35, 45, 52, and possibly 58; all hrHPV types with a close phylogenetic relation to HPV16 or HPV18. The VE was negative for HPV59, but this result is unreliable due to a test artefact leading to many missed infections especially among unvaccinated women [4]. In Chapter 4, the VE was estimated against anal HPV positivity in women, which was high against the vaccine types HPV16 and HPV18. Statistically significant cross-protection was observed for HPV types 31 and 45. Moreover, the level of protection against anal HPV positivity correlated well with protection against genital HPV positivity.

In Part 2 of this thesis, we measured the impact of the HPV vaccination program in the Netherlands on the HPV prevalence using PASSYON study data. We did this by comparing the HPV prevalence across the post-vaccination years with the pre-vaccination year and by studying trends in the HPV prevalence over time. Specifically focusing on the unvaccinated individuals enabled us to measure the indirect effects, i.e. herd effects of vaccination. One of the assumptions of using this approach, is that all factors relevant for transmission remain constant over time. This assumption is violated if there are, for example, secular trends in the targeted pathogen types or if risk factors change over time. Unfortunately, there was only 1 pre-vaccination year and therefore secular trends prior to introduction of vaccination could not be assessed. Secular trends since vaccine introduction are difficult to disentangle from trends induced by vaccination, especially as the risk profile of PASSYON study participants did not remain constant. Information of the participants included in the PASSYON study was used to adjust for changes in risk factors over time. In Chapter 5, decreases in the HPV16 and HPV18 prevalence were observed among women and heterosexual men using data up to 6 years post-vaccination (2009-2015). Among unvaccinated women specifically, such decreases were not observed. The comparison of the HPV prevalence over time was hampered by a policy change at the SHCs in the year 2015, likely leading to a higher HPV prevalence at the 2015 study round. If we were unable to fully adjust for the policy change by correcting for known changes in risk behavior, we likely underestimated the impact of the HPV vaccination program on the HPV16/18 
prevalence. In Chapter 6, we updated the analyses including all measured HPV types and data up to 8 years post-vaccination (2009-2017). With more data available, we were able to additionally adjust for the policy change at the SHCs. Decreases in the HPV prevalence over time were observed for HPV types 16, 18, 31, and 45 among all women; for HPV types 16, 18 , and 31 among heterosexual men; and for HPV types 16 and 18 among unvaccinated women. Increasing trends were observed for HPV56 among all women and for HPV52 among unvaccinated women. Trends in the HPV prevalence up to 8 years post-vaccination among men who have sex with men (MSM) were assessed in Chapter 7. In this study, no declining trends in HPV prevalence were observed.

Taken together, the results described here demonstrate how the post-vaccination dynamics of HPV unfold as the combined result of direct protection afforded to vaccinated women and herd effects in unvaccinated heterosexual men and women. As shown in Figure 1.3 of the general introduction, the population-level impact of vaccination is dependent on the VE. Still, a good correlation between the trend in HPV prevalence and VE is not evident, as there could be test artefacts or secular trends independent of HPV vaccination. Also typereplacement could results in a poor correlation between the trend in HPV over time and VE. In Figure 10.1, we combined the VE estimates of the hrHPV types as measured in Chapter 3 , with the trends in HPV prevalence up to 8 years post-vaccination as measured in Chapter 6. The impact of HPV vaccination among women highly correlated with the VE (Spearman's $\rho=-0.87)$, indicating that there are limited signs of residual secular trends or typereplacement and that our trends measures likely represent the population-level impact due to vaccination. It also shows the consistency of our findings and indicates that both measures of trends and VE are likely to be quite accurate. The correlation between VE and the trends in HPV prevalence became less for the more distant impact measure among heterosexual men and statistically non-significant among unvaccinated women, but this will likely become stronger with longer follow-up. 

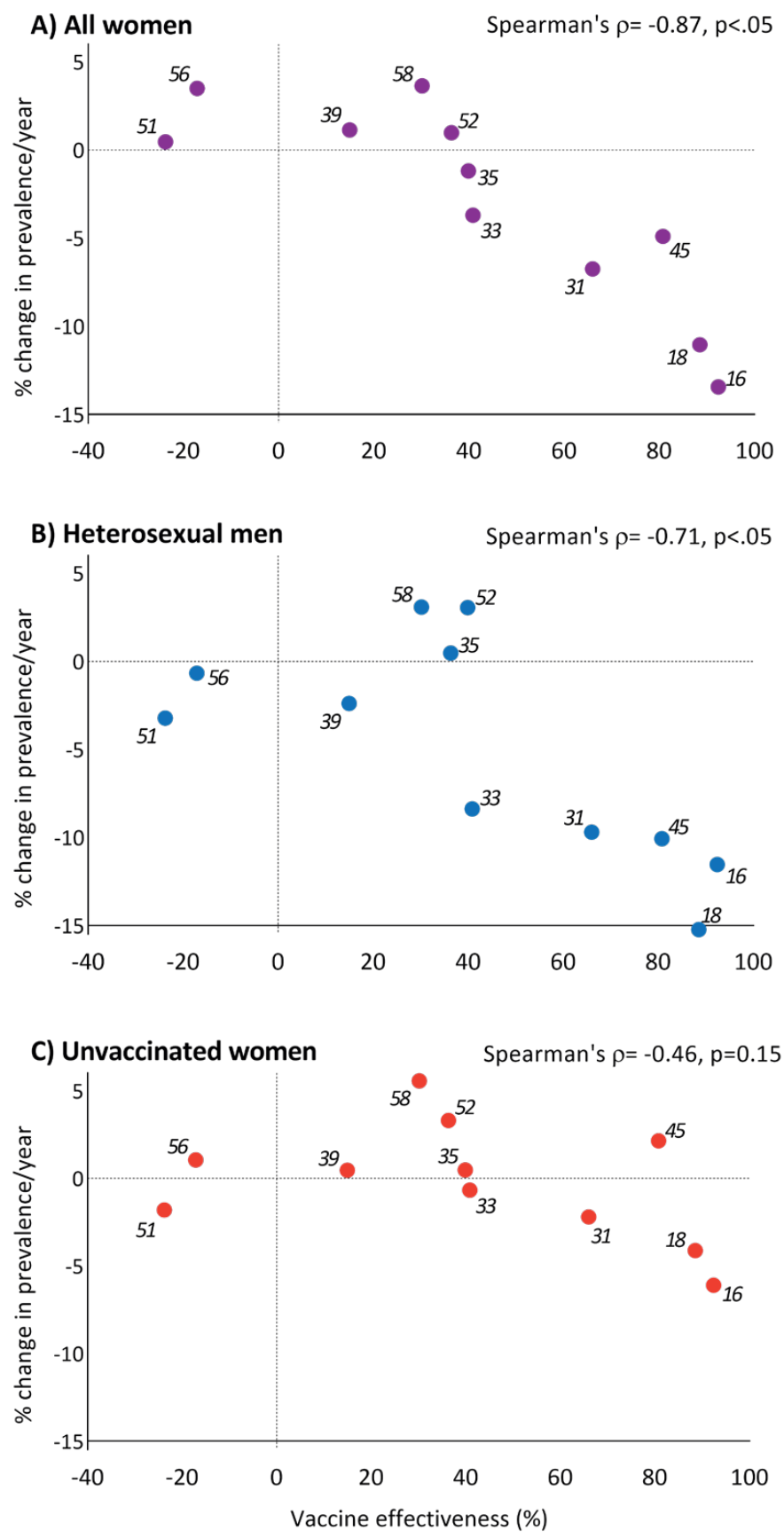

Figure 10.1. Correlation between the percentual change in HPV prevalence per year and the VE for hrHPV types.

Note: This graph combines the results from Chapter 3 (VE against high-risk types) and Chapter 6 (trends in high-risk HPV prevalence up to 8 years post-vaccination). The VE and percentual change in prevalence per year are presented for hrHPV types (indicated in the Figure), excluding HPV59 because of unreliable test results [4]. 
The VE against the 2VHPV vaccine types 16 and 18 as measured in our studies, were comparably high as measured in randomized controlled trials (RCTs) [5] and other observational studies [6-8]. This high VE likely resulted in the decline in HPV16 and HPV18 prevalence over time among all women, demonstrating the population-level impact. Up to 6 year post-vaccination, this decline was explained by an increasing proportion of women who were directly protected by vaccination. As predicted in modeling studies on the transmission of HPV infections and the effects of HPV vaccination [9], we also observed decreases in the HPV16/18 prevalence among heterosexual men within 6 years after girlsonly HPV vaccination, likely as a result of a decreasing force of infection due to vaccination (herd effects). The lower HPV16/18 prevalence among heterosexual men with a steady partner who had been eligible for HPV vaccination, as compared to heterosexual men with a steady partner who had not been eligible for HPV vaccination, underlined that herd effects were occurring. However, these herd effects did not yet reach unvaccinated women, indicated by the lack of a decreasing trend in this population. With longer follow-up time ( 8 years post-vaccination), also decreases in the HPV16/18 prevalence were observed among unvaccinated women. Such temporal unfolding of herd effects is to be expected, as heterosexual men are protected via vaccinated women (first-order herd effects), while heterosexual men in turn protect the unvaccinated women (second-order herd effects in heterosexual transmission). Likewise, herd effects from girls-only vaccination are expected to be less among MSM. Indeed, in Chapter 7 we did not observe evidence for herd effects among MSM. However, this does not necessarily mean there will be no effects among MSM. It is likely that there will be some spillover effects from the heterosexual network as some MSM also have sex with women [10]. This is also demonstrated by a study in Australia, where a small but significant decline was observed in the AGW prevalence among MSM after introduction of girls-only 4vHPV vaccination [11]. Perhaps the sample size and the time since vaccination were not adequate to observe declining trends in the HPV prevalence in our study. On the other hand, spillover effects could also be neutralized by the high sexual risk behavior of the MSM population visiting the SHC [12]. In any case, our studies show that MSM benefit much less from the girls-only vaccination program as compared to heterosexual men.

The non-vaccine targeted types for which we observed cross-protection were also generally in line with results from other studies, although we did observe cross-protection for specific types that others did not or vice versa [5-8]. Factors that influence for which types protection is found in a study include chance, the level of protection, and the occurrence in the study population. The last 2 determine power to find a statistically significant effect. Since the PASSYON study included a high-risk population of visitors of SHCs, the prevalence of HPV was high [13]. This high prevalence in combination with 
efficient statistical methods including both fixed and random effects (mixed effects model), increased both precision and power in our estimation of the VE against HPV types. The high correlation between the level of protection against specific HPV types and the phylogenetic distance of these types towards the vaccine types, supports the cross-protection found in our studies. The cross-protection also translated to herd effects among heterosexual men for these types, but only after 8 years post-vaccination. The longer time it took before herd protection against the cross-protective types became measurable (as compared to the vaccine types) is likely related to a lower VE against cross-protective types and/or a lower prevalence in the population. For example, the declining trend for cross-protective type HPV45 among heterosexual men did not reach statistical significance, probably due to its low prevalence. With more data and longer follow-up time, measurable herd effects for other cross-protective types and among unvaccinated women are expected.

Other than the level of VE there are other factors that influence the population-level impact, of which the vaccination coverage is an important one. A high vaccination coverage is key for an effective vaccination program and a high population-level impact [14]. So far, the Netherlands have experienced a suboptimal vaccination coverage of less than $60 \%$ [15, 16]. Despite the limited vaccination coverage, we showed that even in a high-risk study population, herd effects among heterosexual men and unvaccinated women are present. As it is generally more difficult to reduce the prevalence in a population with a high baseline prevalence [12], the demonstration of herd effects in a high-risk population is encouraging for the population-level impact in the general population, which is likely to be higher. Although herd effects were present, unvaccinated individuals, i.e. about half of the women and all men in the current girls-only vaccination program in the Netherlands, remain susceptible for HPV infection. In countries with a higher uptake (for example Scotland and Australia with both $>80 \%$ uptake), the overall impact and herd effects have been higher or experienced sooner after introduction of vaccination, even with signs of herd effects for cross-protective types among unvaccinated women $[6,17]$. To eliminate HPV types 16 and 18 in the heterosexual population, a sex-neutral vaccination program with over $80 \%$ uptake in both men and women is warranted [18].

Neither the results of this thesis nor the results from other studies provide clear signs of type-replacement within the first 10 years after the introduction of HPV vaccination programs. Just like we reported in Chapter 6, some studies worldwide have experienced increases in specific HPV types over time, but a specific pattern is currently lacking when combining these results [19-22]. Continued monitoring of the prevalence of HPV over time remains important, especially since type-replacement can become apparent many years after the start of vaccination [23]. 
Results from Chapter 4 suggest comparable VE of the 2VHPV vaccine against anal as against genital infections. The PASSYON study can also be used to calculate the VE against oral HPV positivity, by taking oral rinse samples from participants. Other studies have already shown a high VE against HPV vaccine (and cross-protective) types at the oral site, although good effectiveness measures on a type-specific level are still lacking [24, 25]. Because of the high VE of HPV vaccination against the HPV vaccine types at the anal and oral sites, also reductions in the anal and oral HPV prevalence over time are expected in the population. This was already observed, for example in the United States, for oral HPV among heterosexual men [26].

\section{Effects on anogenital warts}

In Part 3 of this thesis, we measured the VE of 2vHPV vaccination against AGWs. In Chapter 8 , we used data from the PASSYON study. In this study, we did not observe an effect of 2vHPV vaccination against HPV6/11; the types most often associated with AGWs [27]. Moreover, the lower AGW prevalence among $2 \mathrm{vHPV}$ vaccinated compared to unvaccinated women was statistically non-significant. In Chapter 9, we used registry data from general practitioners (GPs) to increase power, because in the Netherlands $>95 \%$ of all AGWs are being diagnosed by GPs [28]. These data were linked to the national vaccination registry to obtain the HPV vaccination status, and to data on migration background and educational level to adjust for potential confounders between vaccinated and unvaccinated women. Because registry data were used, there was no information on risk behavior to adjust for. In this study, we observed a statistically significant lower AGW incidence among 2vHPV vaccinated compared to unvaccinated women.

The results of our studies are hinting towards partial protection of $2 \mathrm{vHPV}$ vaccination against AGWs (Chapter 9), but not against HPV6/11 (Chapters 3, 4, 8). Literature regarding the effect of $2 \mathrm{vHPV}$ vaccination against AGWs or HPV6/11 has been equivocal, with some studies finding evidence for an effect [29-32] and others not [7, 8, 21, 33-37]. Observational studies like the ones described in this thesis do not provide definitive answers whether 2 vHPV vaccination directly protects against AGWs, because of the risk of confounding. One way of limiting confounding is by randomly assigning women to receive the HPV or placebo vaccine, as is being done in an RCT. Unfortunately, in the RCTs of the 2vHPV vaccine, vaccine efficacy against AGWs was not assessed [38]. Because it would be unethical to administer a placebo vaccine when the HPV vaccine is known to protect against HPV-related (pre)cancers, observational studies are the only option at this stage to further unravel the VE of 2 vHPV against AGWs. From Chapter 9 we cannot conclude a causal relation because we had limited information on possible confounders, still a direct effect of 2vHPV vaccination against $A G W s$ was supported by the dose-response relation and the lower VE among women who were older when vaccination was offered. Both results are in line with 
other studies assessing the effects of HPV vaccination; like the lower VE against HPV vaccine types among women who were older or sexually experienced when vaccinated $[6,39,40]$ or after an incomplete vaccination course [41, 42]. To limit confounding in future observational studies, detailed information on possible confounding factors such as risk behavior, should be collected to adjust for in the analyses. Also, studies in different settings and different study populations could contribute to evidence of a true (causal) direct effect. Moreover, the possible biological mechanisms of the effect can be studied, like the presence of neutralizing antibodies or T-cell responses cross-reacting with HPV6/11 after $2 \mathrm{vHPV}$ vaccination [43] or the role of hrHPV types in the development of AGWs [44-46].

\section{Future perspective}

Our results should be viewed in context of other monitoring studies measuring the effects of HPV vaccination, like: cohort studies measuring the VE against persistent infections [7]; studies focusing on possible side effects of vaccination [47]; studies analyzing the costeffectiveness [48]; modeling studies predicting effects of (changes in) the vaccination program [49] or the possibility of type-replacement [23]; studies focusing on the effects of vaccination on the molecular characterization of HPV infections such as viral load [50] and genetic diversity [51, 52]; studies on the immune response [53]; and other registry-based (linkage) studies to measure the VE and impact of HPV vaccination on clinical outcomes [54]. Together, these studies provide evidence on the "real-world" effects of HPV vaccination in the Netherlands, which so far are highly beneficial from a public health perspective. Continuation of these studies will demonstrate the long-term effects of vaccination and help to assess the effects of changes in the HPV vaccination program.

\section{2-dose vaccination schedule}

The results described in this thesis mainly represent the effects of the original 3-dose schedule. The 2-dose schedule has been in place since 2014 and girls that were offered 2 doses of HPV vaccine ( $\leq 16$ years old in 2017) constituted only a very small part of our study population. As these girls were still quite young, they likely have not yet contributed to the transmission of HPV to a large extent. For the estimation of VE we mainly focused on women who reported $\geq 1$ dose of HPV vaccine, because many women had trouble recalling the number of doses. It is likely that most of these women had received 3 doses, because in the Netherlands most women who start HPV vaccination also complete the vaccination course [55]. We showed that the self-reported vaccination status ( $\geq 1$ dose) is quite reliable, and sensitivity analyses only including women who reported 3 doses yielded comparable results.

There are post-licensure studies that have measured the VE for 2 doses of the $2 \mathrm{VHPV}$ or quadrivalent (4vHPV) vaccine, but at the time of writing, only within the context of the 3 - 
dose schedule. Most of these studies measured a statistically significant VE against HPV vaccine types or AGWs, but in some studies this effect was less than after 3 doses $[56,57]$. Measuring the VE after incomplete vaccination comes with a high risk of bias. Therefore, results of these studies are not representative for the currently prescribed 2 doses given 6 months apart, probably underestimating the VE. Other than immunogenicity $[58,59]$, there are currently no post-licensure studies that have evaluated the 2-dose vaccination schedule and this remains to be studied. It remains especially to be seen if the VE against crossprotective types is maintained with $<3$ doses of $2 \mathrm{vHPV}$ vaccine. This is currently being evaluated in a prospective cohort study in the Netherlands [60]. Post-hoc analyses from RCTs are reassuring and indicate prolonged (cross-) protection even after 1 dose of HPV vaccine, however this was also performed in the context of the 3-dose schedule [61].

\section{HPV-related diseases}

The PASSYON study was setup to monitor the effects of vaccination on HPV infections soon after vaccine introduction. Within a decade, we showed the beneficial effects of 2vHPV vaccination against HPV positivity of the vaccine and cross-protective types, which is expected to translate into the prevention of HPV-related (pre)cancers; the ultimate goal of the $2 \mathrm{vHPV}$ vaccination program. With the long time to develop cancers after acquiring an HPV infection [62], determination of the VE against HPV-related diseases requires much longer follow-up than considered in this thesis.

A cervical cancer screening program leads to relatively early detection of cervical (pre)cancers. Screening data can be used to evaluate the effects of HPV vaccination against clinical outcomes. Either by studying trends in cervical (pre)cancers over time to measure the population-level impact or by linking screening test results to the vaccination status to measure the VE. Both type of studies have been performed in countries where women are screened at a relatively young age (e.g. from the age of 20 years); all adding to the compelling evidence of the highly beneficial effects of HPV vaccination against clinical outcomes [63-66]. For example, in Scotland a VE of $86 \%$ was observed against cervical intraepithelial neoplasia grade 3 or worse $(\mathrm{CIN} 3+)$ in females fully vaccinated at 12-13 years of age with the $2 \mathrm{vHPV}$ vaccine. In this study also signs of herd effects among unvaccinated women were observed [64]. In the Netherlands, cervical screening starts at the age of 30 years and the first cohort of women who had been eligible for HPV vaccination (birth cohort 1993) will enter the screening program in 2023. From that time onward, declines in (pre)cancers detected in the screening program are also expected for the Netherlands. By linking results from opportunistic screening (women screened at a younger age on indication) to the vaccination status, the first VE estimates of $2 \mathrm{vHPV}$ vaccination against clinical outcomes have been obtained in the Netherlands (preliminary VE of $46 \%$ against CIN2+) [54]. Continued surveillance of the screening results will provide more evidence 
about the effectiveness of HPV vaccination against cervical (pre)cancers. For the cancers at other anatomic sites, the Dutch cancer registries can be used [67]. By studying trends in HPV-related cancer incidence over time, the impact of the HPV vaccination program on cancers, for example at the anal site, can be studied in the long run. However, this will take decades and it will be challenging to distinguish vaccine effects from secular trends highlighting the importance of studies like the PASSYON study, that actively measure the HPV prevalence to monitor the effects of vaccination on HPV infection dynamics soon after vaccine introduction.

A clinical outcome that takes less time to develop are AGWs [68]. Countries that had implemented 4VHPV vaccination, have seen large declines in AGWs soon after vaccine introduction $[63,69,70]$. In the Netherlands, general practice data can be used to measure the population-level impact of HPV vaccination against AGWs, by studying trends in the AGW incidence over time from pre-vaccination to post-vaccination. Up to 8 years post $2 \mathrm{vHPV}$ vaccine introduction there are no clear indications the AGW incidence at the general practice is declining over time among <25-year-olds in the Netherlands [28]. However, this age category might be too wide to observe a population-level impact on AGWs and studying a younger population would be preferred to demonstrate possible early vaccine effects.

\section{The upcoming HPV vaccination program}

From 2021 onwards, HPV vaccination will be offered at the age of 9 years instead of 12 years which is currently the case [2]. At the moment there are no signs of waning of immunity $[71,72]$ or decreases in VE over time [6, 7] (Chapter 3), ensuring a high effectiveness when the vaccinated 9-year-olds reach the age of sexual activity. Yet, this remains important to monitor as the reduced transmission of HPV due to vaccination might take away a natural booster to the vaccine-induced immunity.

The Netherlands will also include boys in the HPV vaccination program in addition to girls [2]. For the 2vHPV vaccine, only the immunogenicity and not the vaccine efficacy was tested in RCTs among men [73, 74], highlighting the importance of evaluating the VE of $2 \mathrm{vHPV}$ vaccination among boys. A small study in Finland showed a reduced prevalence of HPV vaccine types in urine samples among $2 \mathrm{VHPV}$ vaccinated compared to unvaccinated men, indicating first signs of VE [75]. Although the VE among boys is not expected to be different from girls, more studies are warranted. Evaluating the VE of HPV vaccination among boys can be performed in a similar fashion as has been done for girls, for example in a cohort study [7] or as described in Part 1 of this thesis. An advantage of a cohort study, is that VE can be estimated against persistent HPV infection; an outcome measure that is endorsed by the WHO as an intermediate endpoint in the evaluation of HPV vaccination [76]. A disadvantage of a cohort study is that it requires repeated follow-up of both vaccinated and unvaccinated individuals. We have shown that the VE against HPV positivity 
(as measured in the PASSYON study among women), agrees very well with other measures of VE against HPV (persistent) infection, suggesting reliable estimates. A possible challenge in measuring the VE among boys are the herd effects that have been generated by girls-only vaccination; even among unvaccinated heterosexual men the HPV prevalence will be relatively low, leading to limited power. Especially in countries with a high girls-only vaccine uptake and strong herd effects this can be troublesome [77]. With the hitherto limited girlsonly vaccine uptake, the Netherlands could reach an increased population-level impact after switching to a sex-neutral vaccination program, particularly among unvaccinated women and MSM [78]. The PASSYON study provides an important and unique surveillance system to monitor the additional impact of including boys to the program, as it includes data of men and women from both pre- girls-only and pre- sex-neutral vaccination periods [79]. A shift in the trend in HPV prevalence after switching to sex-neutral vaccination, could indicate the additional benefits. Focusing on unvaccinated individuals, measuring herd effects among both men and women will still be possible.

The third recommendation of the Dutch Health Council is to offer free of charge HPV vaccination to men and women $\leq 26$ years of age [2]. Multi-cohort catch-up vaccination is predicted to accelerate the population-level impact of HPV vaccination programs and could compensate for the relatively low vaccine uptake the Netherlands has experienced $[49,80$ 83]. As acknowledged by the Health Council, evaluation of the catch-up vaccination is of importance as the VE will generally be lower with a higher age at vaccination, related to prior HPV exposure $[6,84]$. This is partly because prophylactic HPV vaccination has no effect on (possibly latent) HPV infections prevalent at the moment of vaccination [85] and partly because of the possible build-up of natural immunity, which seems to play a role mainly among women [86]. Whether catch-up HPV vaccination will be beneficial depends on the risk of acquiring an HPV infection after being vaccinated. If this risk is low, also the potential benefits of vaccination will be low. In evaluating the catch-up program, it will be of importance to have information on the age at HPV vaccination, the HPV status (DNA and antibody levels) at the moment of vaccination, and risk behavior.

Catch-up vaccination for 18- to 26-year-olds in the Netherlands will likely not be an active program on a national level [3]. However, the possibility to offer vaccination to previously unvaccinated $\leq 26$-year-olds, does enable to actively target people for HPV vaccination. For example SHCs, where sexual behavior is discussed anyway, could play a major role in promoting HPV vaccination to young visitors who have not been HPV vaccinated. While SHCs could play a role in promoting HPV vaccination to all visitors aged $\leq 26$ years, a target population for which HPV vaccination might be beneficial are MSM; a population with a high HPV-related disease burden who have not yet benefitted from the girls-only HPV vaccination program $[49,87]$. In trials of the $4 \mathrm{vHPV}$ vaccine, vaccine efficacy 
was high among 16- to 26-year-old MSM who were HPV DNA negative and HPV seronegative $[88,89]$. Previous research indicated high intention to vaccinate against HPV among MSM visiting the SHC of Amsterdam [90]. In the United Kingdom, where targeted $H P V$ vaccination is already implemented for MSM $\leq 45$ years of age visiting SHCs, the vaccine uptake varied between nearly $50 \%$ in England to nearly $65 \%$ in Scotland, showing the feasibility of such a program [91-93]. Many MSM may remain being exposed to HPV during their lifetime [94] and these future infections could potentially be prevented by prophylactic vaccination. In Chapter 7 of this thesis, we showed that the majority of the 16- to 24-yearold MSM visiting SHCs, were HPV DNA negative and HPV seronegative for the various vaccine types, suggesting that the high vaccine efficacy of the trial setting could apply. However, a high VE among this target population is not straightforward. One major difference between the MSM population visiting the SHCs in the Netherlands and the MSM included in the RCTs to assess vaccine efficacy, is the number of lifetime sex partners [88, 89]. In the trials, this was limited to 5 , while $>80 \%$ of the young MSM in our study had $>5$ lifetime sex partners. Because the VE is dependent on prior HPV exposure, the actual VE in MSM attending SHCs is currently unknown and should be evaluated [95]. A targeted HPV vaccination program could also be extended to other groups at continued high risk of HPV infections, like sex workers. Also in this case, a high VE is not straightforward and should be evaluated [96].

\section{Concluding remarks}

In Chapter 1 of this thesis, we introduced a theoretical framework to distinguish and measure direct and indirect effects of vaccination. The direct effects reflect the protection of vaccinated individuals while the indirect effects reflect the changes in the transmission dynamics as a result of vaccination. The HPV vaccination program in the Netherlands has offered an unique case study to measure the indirect herd effects, as vaccination was offered to girls only with a suboptimal vaccination coverage. By studying trends in HPV prevalence among unvaccinated men and women, this thesis builds on the theoretical framework of vaccine effects by demonstrating first- and second-order herd effects and how these unfold over time.

We measured the effects of 2 VHPV vaccination on HPV positivity and AGWs. The results add to the growing body of evidence on the highly beneficial effects of HPV vaccination. The VE against the vaccine types was high at the genital as well as the anal site. Moreover, there was cross-protection against non-vaccine HPV types, especially for those types phylogenetically closely related to the vaccine types. Although no cross-protection against low-risk types was observed, AGWs were less often diagnosed among 2 vHPV vaccinated 
women. Decreases in the vaccine-type HPV prevalence were observed among heterosexual men after 6 years post-vaccination and among unvaccinated women after 8 years postvaccination. These results reflect the first- and second-order herd effects in a girls-only vaccination program for a sexually transmitted infection. Measurable herd effects among MSM were not observed. The PASSYON study, in which the HPV prevalence was repeatedly and consistently measured among the high-risk population of visitors of SHCs, has proven to be very valuable and efficient in measuring several aspects of vaccine effects within a decade after vaccine introduction. The study contains unique HPV prevalence data from pre- to post-vaccination periods among both vaccinated and unvaccinated women and men. The PASSYON study should be continued to keep on monitoring the "real-world" evidence of HPV vaccination and the effects of the upcoming changes in the program.

\section{References}

1. Donken R, et al. Human papillomavirus (HPV) infection. In: Schurink-van 't Klooster TM, de Melker $\mathrm{HE}$, eds. The National Immunisation Programme in the Netherlands - Surveillance and developments in 2013-2014. Bilthoven: RIVM, 2014:77-85.

2. Gezondheidsraad. [Vaccinatie tegen HPV]. Den Haag: Gezondheidsraad, 2019. Nr: 2019/09.

3. Blokhuis P. [Uitbreiding vaccinatie tegen door HPV veroorzaakte kanker]. 2019. Nr: 32793-446.

4. Leussink S, et al. Comparison of SPF10-DEIA-LIPA system version 1 with type-specific qPCR for detection of HPV59 infections, evidence for missing HPV59 infections and impact on vaccine effectiveness measurements. The 32 nd International Papillomavirus Conference 2018.

5. Skinner SR, et al. Human papillomavirus (HPV)16/18 AS04-adjuvanted vaccine for the prevention of cervical cancer and HPV-related diseases. Expert Rev Vaccines 2016; 15:367-87.

6. Kavanagh $\mathrm{K}$, et al. Changes in the prevalence of human papillomavirus following a national bivalent human papillomavirus vaccination programme in Scotland: a 7-year cross-sectional study. Lancet Infect Dis 2017; 17:1293-302.

7. Donken R, et al. High Effectiveness of the Bivalent Human Papillomavirus (HPV) Vaccine Against Incident and Persistent HPV Infections up to 6 Years After Vaccination in Young Dutch Women. J Infect Dis 2018; 217:1579-89.
8. Lehtinen $M$, et al. Gender-neutral vaccination provides improved control of human papillomavirus types 18/31/33/35 through herd immunity: Results of a community randomized trial (III). Int J Cancer 2018; 143:2299-310.

9. Garnett GP. Role of herd immunity in determining the effect of vaccines against sexually transmitted disease. J Infect Dis 2005; 191 Suppl 1:S97-106.

10. de Graaf $\mathrm{H}$, et al. [Seks onder je 25e]. Utrecht: Rutgers, Soa aids Nederland, 2017.

11. Chow EP, et al. Ongoing decline in genital warts among young heterosexuals 7 years after the Australian human papillomavirus (HPV) vaccination programme. Sex Transm Infect 2015; 91:214-9.

12. Baussano I, et al. Impacts of human papillomavirus vaccination for different populations: A modeling study. Int J Cancer 2018; 143:1086-92.

13. Mollers $M$, et al. Population- and type-specific clustering of multiple HPV types across diverse risk populations in the Netherlands. Am J Epidemiol 2014; 179:1236-46.

14. Malagon $\mathrm{T}$, et al. Human papillomavirus vaccination and the role of herd effects in future cancer control planning: a review. Expert Rev Vaccines 2018; 17:395-409.

15. van Lier EA, et al. [Vaccinatiegraad Rijksvaccinatieprogramma Nederland: Verslagjaar 2011]. Bilthoven: RIVM, 2011. Nr: 210021014/2011. 
16. van Lier EA, et al. [Vaccinatiegraad en jaarverslag Rijksvaccinatieprogramma Nederland 2018]. Bilthoven: RIVM, 2019. Nr: 2019-0015.

17. Tabrizi SN, et al. Assessment of herd immunity and cross-protection after a human papillomavirus vaccination programme in Australia: a repeat crosssectional study. Lancet Infect Dis 2014; 14:958-66.

18. Brisson $M$, et al. Population-level impact, herd immunity, and elimination after human papillomavirus vaccination: a systematic review and meta-analysis of predictions from transmissiondynamic models. Lancet Public Health 2016; 1:e8e17.

19. Mesher D, et al. Population-Level Effects of Human Papillomavirus Vaccination Programs on Infections with Nonvaccine Genotypes. Emerg Infect Dis 2016; 22:1732-40.

20. Gray P, et al. Evaluation of HPV type-replacement in unvaccinated and vaccinated adolescent femalesPost-hoc analysis of a community-randomized clinical trial (II). Int J Cancer 2018; 142:2491-500.

21. Mesher D, et al. The Impact of the National HPV Vaccination Program in England Using the Bivalent HPV Vaccine: Surveillance of Type-Specific HPV in Young Females, 2010-2016. J Infect Dis 2018; 218:911-21.

22. Feiring B, et al. Substantial Decline in Prevalence of Vaccine-Type and Nonvaccine-Type Human Papillomavirus (HPV) in Vaccinated and Unvaccinated Girls 5 Years After Implementing HPV Vaccine in Norway. J Infect Dis 2018; 218:1900-10.

23. Man I, et al. Human papillomavirus genotype replacement: still too early to tell? J Infect Dis 2020.

24. Schlecht NF, et al. Risk of Oral Human Papillomavirus Infection Among Sexually Active Female Adolescents Receiving the Quadrivalent Vaccine. JAMA Netw Open 2019; 2:e1914031.

25. Lehtinen $M$, et al. Effectiveness of the ASO4adjuvanted HPV-16/18 vaccine in reducing oropharyngeal HPV infections in young femalesResults from a community-randomized trial. Int J Cancer 2020; 147:170-4.

26. Chaturvedi AK, et al. Prevalence of Oral HPV Infection in Unvaccinated Men and Women in the United States, 2009-2016. JAMA 2019; 322:977-9.

27. Garland SM, et al. Natural history of genital warts: analysis of the placebo arm of 2 randomized phase III trials of a quadrivalent human papillomavirus (types 6, 11, 16, and 18) vaccine. J Infect Dis 2009; 199:805-14.

28. Slurink IAL, et al. Sexually transmitted infections in the Netherlands in 2018. Bitlhoven: RIVM, 2019. Nr: 2019-0007; DOI: 10.21945/RIVM-2019-0007.

29. Howell-Jones R, et al. Declining Genital Warts in Young Women in England Associated With HPV 16/18 Vaccination: An Ecological Study. J Infect Dis 2013; 208:1397-403.

30. Canvin $M$, et al. Decline in genital warts diagnoses among young women and young men since the introduction of the bivalent HPV (16/18) vaccination programme in England: an ecological analysis. Sex Transm Infect 2017; 93:125-8.

31. Tota JE, et al. Efficacy of the ASO4-adjuvanted HPV-16/18 vaccine: Pooled analysis of the Costa Rica Vaccine and PATRICIA randomized controlled trials. J Natl Cancer Inst 2019.

32. Latsuzbaia A, et al. Effectiveness of bivalent and quadrivalent human papillomavirus vaccination in Luxembourg. Cancer Epidemiol 2019; 63:101593.

33. Sonnenberg $P$, et al. Epidemiology of genital warts in the British population: implications for HPV vaccination programmes. Sex Transm Infect 2019; 95:386-90.

34. Petras $M$, et al. Impact of quadrivalent human papillomavirus vaccine in women at increased risk of genital warts burden: Population-based crosssectional survey of Czech women aged 16 to 40 years. Vaccine 2015; 33:6264-7.

35. Navarro-Illana E, et al. Effectiveness of HPV vaccines against genital warts in women from Valencia, Spain. Vaccine 2017; 35:3342-6.

36. Cameron RL, et al. Human Papillomavirus Prevalence and Herd Immunity after Introduction of Vaccination Program, Scotland, 2009-2013. Emerg Infect Dis 2016; 22:56-64.

37. Tanton C, et al. Human papillomavirus (HPV) in young women in Britain: Population-based evidence of the effectiveness of the bivalent immunisation programme and burden of quadrivalent and 9-valent vaccine types. Papillomavirus Res 2017; 3:36-41.

38. Szarewski A, et al. Efficacy of the HPV-16/18 ASO4-adjuvanted vaccine against low-risk HPV types (PATRICIA randomized trial): an unexpected observation. J Infect Dis 2013; 208:1391-6. 
39. Woestenberg PJ, et al. Bivalent Vaccine Effectiveness Against Type-Specific HPV Positivity: Evidence for Cross-Protection Against Oncogenic Types Among Dutch STI Clinic Visitors. J Infect Dis 2018; 217:213-22.

40. Lehtinen $M$, et al. Impact of gender-neutral or girls-only vaccination against human papillomavirusResults of a community-randomized clinical trial (I). Int J Cancer 2018; 142:949-58.

41. Herweijer $E$, et al. Association of varying number of doses of quadrivalent human papillomavirus vaccine with incidence of condyloma. JAMA 2014; 311:597-603.

42. Blomberg $M$, et al. Dose-Related Differences in Effectiveness of Human Papillomavirus Vaccination Against Genital Warts: A Nationwide Study of 550,000 Young Girls. Clin Infect Dis 2015; 61:676-82.

43. Evans TG, et al. A Phase 1 study of a recombinant viruslike particle vaccine against human papillomavirus type 11 in healthy adult volunteers. J Infect Dis 2001; 183:1485-93.

44. Ball SL, et al. Analyses of human papillomavirus genotypes and viral loads in anogenital warts. J Med Virol 2011; 83:1345-50.

45. Hawkins MG, et al. Detection of specific HPV subtypes responsible for the pathogenesis of condylomata acuminata. Virol J 2013; 10:137.

46. Hasanzadeh $M$, et al. The interaction of high and low-risk human papillomavirus genotypes increases the risk of developing genital warts: A populationbased cohort study. J Cell Biochem 2019; 120:128704.

47. Schurink-van 't Klooster TM, et al. [Surveillance van mogelijke bijwerkingen na HPV-vaccinatie: het vaccin is veilig]. Infectieziekten Bulletin 2019; 30.

48. Qendri V, et al. Health and Economic Impact of a Tender-Based, Sex-Neutral Human Papillomavirus 16/18 Vaccination Program in the Netherlands. J Infect Dis 2017; 216:210-9.

49. Bogaards JA, et al. Potential effectiveness of prophylactic HPV immunization for men who have sex with men in the Netherlands: A multi-model approach. PLoS Med 2019; 16:e1002756.

50. van der Weele $P$, et al. Effect of the bivalent HPV vaccine on viral load of vaccine and non-vaccine HPV types in incident clearing and persistent infections in young Dutch females. PLoS One 2019; 14:e0212927.
51. King AJ, et al. Genetic Diversity in the Major Capsid L1 Protein of HPV-16 and HPV-18 in the Netherlands. PLoS One 2016; 11:e0152782.

52. Van Eer K, et al. Analyzing the viral properties of human papillomavirus 16 in vaccinated and nonvaccinated women. The 33rd International Papillomavirus Conference 2020. Abstract nr: 556.

53. Pasmans $\mathrm{H}$, et al. Long-term HPV-specific immune response after one versus two and three doses of bivalent HPV vaccination in Dutch girls. Vaccine 2019; 37:7280-8.

54. Schurink-van 't Klooster TM, et al. Effect of HPV vaccination on cervical lesions in oppertunistic cervical screening among young women in the Netherlands. The 33rd International Papillomavirus Conference 2020. Abstract nr: 816.

55. van Lier EA, et al. [Vaccinatiegraad Rijksvaccinatieprogramma Nederland: Verslagjaar 2011]. Bilthoven: RIVM, 2012. Nr: 201001001/2012.

56. Markowitz LE, et al. Human papillomavirus vaccine effectiveness by number of doses: Systematic review of data from national immunization programs. Vaccine 2018; 36:4806-15.

57. Johnson Jones ML, et al. Effectiveness of 1, 2, and 3 Doses of Human Papillomavirus Vaccine Against High-Grade Cervical Lesions Positive for Human Papillomavirus 16 or 18. Am J Epidemiol 2020; 189:265-76.

58. Donken R, et al. Immune Responses After 2 Versus 3 Doses of HPV Vaccination up to 4(1/2) Years After Vaccination: An Observational Study Among Dutch Routinely Vaccinated Girls. J Infect Dis 2017; 215:359-67.

59. Schurink-van 't Klooster TM, et al. Persistence of immune response following bivalent HPV vaccination: A follow-up study among girls routinely vaccinated with a two-dose schedule. Vaccine 2018; 36:7580-7.

60 . Hoes J, et al. Genital HPV prevalence among twodose bivalent HPV vaccine eligible girls in the Netherlands after three years of follow-up: the HAVANA2 cohort. The 33rd International Papillomavirus Conference 2020. Abstract nr: 723.

61. Tsang SH, et al. Durability of Cross-Protection by Different Schedules of the Bivalent HPV Vaccine: the CVT Trial. J Natl Cancer Inst 2020. 
62. Burger EA, et al. Estimating the Natural History of Cervical Carcinogenesis Using Simulation Models: A CISNET Comparative Analysis. J Natl Cancer Inst 2019.

63. Drolet $\mathrm{M}$, et al. Population-level impact and herd effects following the introduction of human papillomavirus vaccination programmes: updated systematic review and meta-analysis. Lancet 2019; 394:497-509.

64. Palmer T, et al. Prevalence of cervical disease at age 20 after immunisation with bivalent HPV vaccine at age 12-13 in Scotland: retrospective population study. BMJ 2019; 365:I1161.

65. Verdoodt F, et al. Dose-related Effectiveness of Quadrivalent Human Papillomavirus Vaccine Against Cervical Intraepithelial Neoplasia: A Danish Nationwide Cohort Study. Clin Infect Dis 2020; 70:608-14.

66. Racey CS, et al. Cervical Intraepithelial Neoplasia Rates in British Columbia Women: A PopulationLevel Data Linkage Evaluation of the School-Based HPV Immunization Program. J Infect Dis 2020; 221:81-90.

67. Netherlands Cancer Registry (NCR). www.cijfersoverkanker.nl. Accessed 24-02-2020.

68. Park IU, et al. Human Papillomavirus and Genital Warts: A Review of the Evidence for the 2015 Centers for Disease Control and Prevention Sexually Transmitted Diseases Treatment Guidelines. Clin Infect Dis 2015; 61 Suppl 8:S849-55.

69. Read TR, et al. The near disappearance of genital warts in young women 4 years after commencing a national human papillomavirus (HPV) vaccination programme. Sex Transm Infect 2011; 87:544-7.

70. Checchi $M$, et al. Declines in anogenital warts diagnoses since the change in 2012 to use the quadrivalent HPV vaccine in England: data to end 2017. Sex Transm Infect 2019; 95:368-73.

71. Artemchuk $\mathrm{H}$, et al. Long-term Antibody Response to Human Papillomavirus Vaccines: Up to 12 Years of Follow-up in the Finnish Maternity Cohort. J Infect Dis 2019; 219:582-9.

72. Hoes J, et al. Persisting Antibody Response 9 Years After Bivalent Human Papillomavirus (HPV) Vaccination in a Cohort of Dutch Women: Immune Response and the Relation to Genital HPV Infections. J Infect Dis 2020; 221:1884-94.
73. Petaja T, et al. Immunogenicity and safety of human papillomavirus (HPV)-16/18 AS04adjuvanted vaccine in healthy boys aged $10-18$ years. J Adolesc Health 2009; 44:33-40.

74. Harder T, et al. Efficacy, effectiveness and safety of vaccination against human papillomavirus in males: a systematic review. BMC Med 2018; 16:110. 75. Lehtinen T, et al. Human Papillomavirus (HPV) Prevalence in Male Adolescents 4 Years After HPV16/18 Vaccination. J Infect Dis 2017; 216:966-8.

76. IARC HPV Working Group. Primary End-points for Prophylactic HPV Vaccine Trials. Lyon (FR) 2014.

77. Chow EPF, et al. Prevalence of human papillomavirus in teenage heterosexual males following the implementation of female and male school-based vaccination in Australia: 2014-2017. Vaccine 2019; 37:6907-14.

78. Qendri V, et al. Who Will Benefit From Expanding HPV Vaccination Programs to Boys? JNCl Cancer Spectrum 2018; 2:pky076.

79. Brotherton JML, et al. Monitoring the impact of HPV vaccine in males-Considerations and challenges. Papillomavirus Res 2016; 2:106-11.

80. Elfstrom KM, et al. Human Papillomavirus Vaccination of Boys and Extended Catch-up Vaccination: Effects on the Resilience of Programs. J Infect Dis 2016; 213:199-205.

81. Matthijsse SM, et al. Public Health Benefits of Routine Human Papillomavirus Vaccination for Adults in the Netherlands: A Mathematical Modeling Study. J Infect Dis 2016; 214:854-61.

82. Drolet $M$, et al. The Impact of Human Papillomavirus Catch-Up Vaccination in Australia: Implications for Introduction of Multiple Age Cohort Vaccination and Postvaccination Data Interpretation. J Infect Dis 2017; 216:1205-9.

83. Orumaa M, et al. The impact of HPV multi-cohort vaccination: Real-world evidence of faster control of HPV-related morbidity. Vaccine 2020; 38:1345-51.

84. Herweijer E, et al. Quadrivalent HPV vaccine effectiveness against high-grade cervical lesions by age at vaccination: A population-based study. Int J Cancer 2016; 138:2867-74.

85. Future II Study Group. Prophylactic efficacy of a quadrivalent human papillomavirus (HPV) vaccine in women with virological evidence of HPV infection. J Infect Dis 2007; 196:1438-46. 
86. Beachler DC, et al. Natural Acquired Immunity Against Subsequent Genital Human Papillomavirus Infection: A Systematic Review and Meta-analysis. J Infect Dis 2016; 213:1444-54.

87. Marra $E$, et al. [Is vaccinatie van jongens of mannen tegen HPV zinvol?]. NTvG 2018; 162:D1200.

88. Giuliano AR, et al. Efficacy of quadrivalent HPV vaccine against HPV Infection and disease in males. N Engl J Med 2011; 364:401-11.

89. Palefsky JM, et al. HPV vaccine against anal HPV infection and anal intraepithelial neoplasia. N Engl J Med 2011; 365:1576-85.

90. Marra $E$, et al. HPV vaccination intention among male clients of a large STI outpatient clinic in Amsterdam, the Netherlands. Papillomavirus Res 2016; 2:178-84.

91. Checchi M, et al. HPV vaccination of gay, bisexual and other men who have sex with men in sexual health and HIV clinics in England: vaccination uptake and attendances during the pilot phase. Sex Transm Infect 2019; 95:608-13.
92. Edelstein $\mathrm{M}$, et al. Implementation and evaluation of the human papillomavirus (HPV) vaccination pilot for men who have sex with men (MSM), England, April 2016 to March 2017. Euro Surveill 2019; 24.

93. Pollock KG, et al. HPV vaccine uptake in men who have sex with men in Scotland. Vaccine 2019; 37:5513-4.

94. Poynten IM, et al. Comparison of age-specific patterns of sexual behaviour and anal HPV prevalence in homosexual men with patterns in women. Sex Transm Infect 2016; 92:228-31.

95. Forster AS, et al. Challenges to optimising uptake and delivery of a HPV vaccination programme for men who have sex with men. Hum Vaccin Immunother 2019; 15:1541-3.

96. Schim van der Loeff MF, et al. Should female sex workers be offered HPV vaccination? Hum Vaccin Immunother 2019; 15:1544-8 




\section{Supplements}

Summary

Nederlandse samenvatting

Valorization

List of abbreviations

Dankwoord

Curriculum vitae

List of publications 


\section{Summary}

An infection with sexually transmitted human papillomavirus (HPV) is usually asymptomatic but can sometimes cause anogenital warts (AGWs) and various anogenital and head-andneck cancers in men and women. Girls-only bivalent HPV (2vHPV) vaccination was introduced in the Netherlands in 2009 to prevent the most oncogenic HPV types 16 and 18. After implementation of vaccines in national immunization programs (NIPs), observational studies are needed to assess the (long-term) effects of vaccination on the occurrence of the infection in a population at large.

Chapter 1 provides an introduction to HPV, HPV-related diseases, the available HPV vaccines, and the implementation of HPV vaccination in the Netherlands. Moreover, it describes a theoretical framework by which different effects of vaccination can be distinguished and how these effects can be measured in post-vaccine surveillance. We highlight the direct vaccine effectiveness (VE) and impact of vaccination. The VE is related to the direct protection of the vaccine itself in a vaccinated person (individual-level effect), while the impact is related to the effects of a vaccination program in the total population (population-level effects). To measure the different effects of HPV vaccination, the PASSYON (PApillomavirus Surveillance among STI clinic YOungsters in the Netherlands) study was set-up. In this cross-sectional study that started in 2009 and was repeated every other year, 16- to 24- year-old men and women visiting sexual health centers (SHCs) throughout the Netherlands were tested for type-specific HPV positivity. The studies described in this thesis aimed to measure the VE of 2VHPV vaccination against HPV positivity and AGWs and to measure the impact of the HPV vaccination program in the Netherlands on the HPV prevalence.

\section{Part 1: Direct vaccine effectiveness against HPV positivity}

The first part of this thesis focuses on the VE of 2VHPV vaccination against HPV positivity, among women in the PASSYON study who had been eligible for HPV vaccination in the Netherlands, i.e. women born in 1993 or later. In Chapter 2, we estimated the VE of the 2 VHPV vaccine against genital oncogenic HPV positivity. We compared HPV positivity between self-reported vaccinated ( $\geq 1$ dose) and unvaccinated women, and estimated VE by a logistic mixed model. The adjusted pooled VE against the vaccine types HPV16/18 was high (90\%, 95\% confidence interval [CI] 82\%-94\%). Moreover, we calculated significant VE against non-vaccine types HPV45 (91\%), HPV35 (57\%), HPV31 (50\%), and HPV52 (37\%). In Chapter 3, we updated the analyses of Chapter 2 using more data and including low-risk HPV types. Moreover, we evaluated the VE against type-specific HPV positivity as a function of phylogenetic distance to vaccine types HPV16/18. 2vHPV was predicted to provide partial 
cross-protection against HPV types $31,33,35,45,52$, and possibly 58; all oncogenic types with close phylogenetic relationships to HPV16 or HPV18. Cross-protection and protection against HPV16/18 was sustained up to 8 years post-vaccination. In Chapter 4 , type-specific VE against HPV positivity at the anal site was measured using the same methods as in Chapter 2. VE against the vaccine types HPV16/18 was high (90\%, 95\% Cl, 63\%-97\%). Moreover, we calculated significant VE against non-vaccine types HPV45 (100\%) and HPV31 (73\%). Type-specific VE against anal HPV correlated well with VE against genital HPV (Spearman's $\rho=0.76$ ), suggesting comparable effectiveness of $2 \mathrm{vHPV}$ vaccination against genital and anal infections.

\section{Part 2: Population-level impact on HPV positivity}

The second part of this thesis focuses on the impact of the HPV vaccination program in the Netherlands on the HPV prevalence, using data from the PASSYON study. In Chapter 5 , we assessed trends in HPV16 and HPV18 prevalence among women and heterosexual men, using data up to 6 years post-vaccination. We compared the genital post-vaccination HPV prevalence with the pre-vaccination prevalence (year 2009) using Poisson GEE models. We observed significantly decreasing trends in the HPV16/18 prevalence among women (from $23 \%$ pre-vaccination to $15 \%$ in 2015 ) and heterosexual men (from $17 \%$ pre-vaccination to $11 \%$ in 2015), but not among unvaccinated women. Of the heterosexual men with a steady partner, the HPV16/18 prevalence was lower among those men whose steady partner had been vaccine-eligible in the NIP. The results strongly suggest that heterosexual men benefit from herd protection from girls-only vaccination. In Chapter 6 , we updated the analyses of Chapter 5 and assessed trends in prevalence of 25 HPV types in the period 2009-2017 (up to 8 years post-vaccination). The HPV16/18 prevalence decreased among women (with $12.6 \%$ annually), heterosexual men (with $13.0 \%$ annually), and unvaccinated women (with 5.4\% annually). Moreover, decreases in HPV31 and HPV45 were observed among women and heterosexual men. Increases were observed in HPV56 among women and in HPV52 among unvaccinated women. The results suggest herd effects among heterosexual men for vaccine and cross-protective HPV types. Additionally, the results suggest second-order herd effects for the vaccine types among unvaccinated women. In Chapter 7, we studied possible herd effects among men who have sex with men (MSM), by assessing trends in HPV prevalence in the period 2009-2017. No declining trends in penile or anal HPV prevalence were observed, indicating that MSM are unlikely to benefit largely from herd effects from girls-only vaccination. Moreover, in this chapter we studied prior exposure to HPV among MSM, by assessing the presence of HPV DNA at the anal and penile site and presence of HPV antibodies. The majority of the MSM were HPV DNA negative at the penile as well as the anal site for all types included in the bivalent (79\%), quadrivalent (62\%), and nonavalent vaccine (53\%). Among MSM who were HPV16/18 and HPV16/18/31/33/45/52/58 DNA 
negative, $82 \%$ and $71 \%$ were also seronegative for the respective types. Because many MSM were vaccine-type DNA negative and seronegative, vaccination of young MSM visiting SHCS could still be beneficial.

\section{Part 3: Direct vaccine effectiveness against anogenital warts}

The third part of this theses focuses on the VE of $2 \mathrm{VHPV}$ vaccination against AGWs among women who had been eligible for HPV vaccination in the Netherlands. In Chapter 8 , we studied the effect of the 2VHPV vaccine on genital HPV6/11 positivity and AGWs, using data from the PASSYON study. Prevalence of HPV6 and/or HPV11 and AGWs were compared between self-reported vaccinated and unvaccinated women by log-binomial regression analysis. We observed no cross-protective effect of the 2 VHPV vaccine on genital HPV6/11 positivity ( $\mathrm{VE}=9 \%, 95 \% \mathrm{Cl}-31 \%-37 \%)$ and a non-significant partially protective effect against AGWs (VE=36\%, 95\% Cl -107\%-78\%). In Chapter 9 we assessed the effect of 2vHPV vaccination on AGWs using data from general practices, where the vast majority of the AGWs are being diagnosed in the Netherlands. By linking general practice data to the Dutch national immunization registry, we performed a retrospective registry-based open cohort study. Adjusted incidence rate ratios (aIRRs) were estimated using Poisson regression with vaccination status as a time-dependent exposure. AGW incidence was lower after $\geq 1$ dose versus 0 doses (aIRR $0.75,95 \% \mathrm{Cl} 0.64-0.88$ ), which corresponds to a VE of $25 \%$. The effect of vaccination was stronger after full vaccination ( $\mathrm{VE}=28 \%$ ) and for women who were offered vaccination at 12-13 years of age (VE=31\%). Although we were unable to adjust for sexual risk behavior in this study, the results seem to suggest that 2vHPV vaccination partially protects against AGWs.

Last, in Chapter 10 the main findings of this thesis are summarized and discussed. Future perspectives for measuring the individual- and population-level effects of HPV vaccination are given, keeping in mind upcoming changes in the HPV vaccination program, like a lower age at vaccine-initiation, sex-neutral vaccination, and multi-cohort catch-up vaccination. The results of this thesis add to the growing body of evidence on the highly beneficial effects of HPV vaccination, on an individual- as well as a population-level. The PASSYON study has proven to be valuable in measuring several aspects of HPV vaccination effects and should be continued to keep on monitoring the effects of HPV vaccination following upcoming changes in the program. 


\section{Nederlandse samenvatting}

Een infectie met het seksueel overdraagbare humaan papillomavirus (HPV) verloopt in de meeste gevallen asymptomatisch, maar kan in sommige gevallen leiden tot anogenitale wratten (AGW), anogenitale kanker, en hoofd-hals kanker in mannen en vrouwen. In Nederland wordt sinds 2009 HPV vaccinatie aangeboden aan meisjes. Hiervoor wordt gebruik gemaakt van het bivalente HPV (2vHPV) vaccin dat bescherming biedt tegen HPV16 en HPV18; de HPV types die de meeste kanker veroorzaken. Na de invoering van vaccinatie in het Rijksvaccinatieprogramma (RVP) zijn observationele studies nodig om het (lange termijn) effect van vaccinatie te meten op het voorkomen van de infectie in de algehele populatie.

Hoofdstuk 1 geeft een introductie over HPV, HPV-gerelateerde ziektes, de verschillende HPV vaccins, en de toevoeging van HPV aan het RVP in Nederland. Daarnaast wordt in Hoofdstuk 1 een theoretisch kader beschreven waarmee verschillende effecten van vaccinatie kunnen worden onderscheiden en hoe deze effecten kunnen worden gemeten in post-vaccinatie surveillance. We bespreken de directe vaccin effectiviteit (VE) en de impact van vaccinatie. De VE is gerelateerd aan de directe bescherming van het vaccin zelf voor een gevaccineerd persoon (individueel effect). De impact is gerelateerd aan de effecten van het gehele vaccinatieprogramma in de bevolking (populatie-effect). Om de verschillende HPV vaccinatie-effecten te meten, werd in 2009 de PASSYON (PApillomavirus Surveillance among STI clinic YOungsters in the Netherlands) studie opgezet. In deze studie wordt eens in de 2 jaar een dwarsdoorsnedeonderzoek uitgevoerd naar de prevalentie van type-specifieke HPV bij $16 \mathrm{t} / \mathrm{m} 24$ jarige bezoekers van de centra seksuele gezondheid (CSG). Het doel van de studies in dit proefschrift was het meten van de VE van het 2 VHPV vaccin tegen HPV positiviteit en AGW en het meten van de impact van het HPV vaccinatieprogramma in Nederland op de HPV prevalentie.

\section{Deel 1: Directe vaccinatie effectiviteit tegen HPV positiviteit}

In het eerste deel van dit proefschrift wordt de VE tegen HPV positiviteit onderzocht binnen vrouwen die in aanmerking kwamen voor HPV vaccinatie in Nederland, namelijk vrouwen geboren in 1993 of later. Er werd gebruik gemaakt van data van de PASSYON studie. In Hoofdstuk 2 hebben we de VE berekend tegen genitale oncogene HPV types. Dit hebben we gedaan door de HPV prevalentie te vergelijken tussen vrouwen die zelf rapporteerden gevaccineerd ( $\geq 1$ dosis) of niet gevaccineerd te zijn. De VE was hoog tegen de vaccintypes HPV16/18 (90\%, 95\% betrouwbaarheidsinterval [BI] 82\%-94\%). Daarnaast was er significante kruisbescherming tegen niet-vaccintypes, namelijk 91\% tegen HPV45, 57\% tegen HPV35, 50\% tegen HPV31, en 37\% tegen HPV52. In $\underline{\text { Hoofdstuk } 3}$ hebben we de 
analyses van Hoofdstuk 2 herhaald met meer gegevens en met niet-oncogene HPV types. Daarnaast hebben we de type-specifieke VE afgezet tegen de fylogenetische afstand naar de vaccintypes HPV16/18. De analyses wezen erop dat het 2vHPV vaccin gedeeltelijke bescherming biedt tegen HPV types $31,33,35,45$, 52, en mogelijk 58. Dit zijn allemaal oncogene HPV types die nauw verwant zijn aan HPV16 of HPV18. De kruisbescherming en de bescherming tegen de vaccintypes bleven hoog tot 8 jaar na vaccinatie. In $\underline{\text { Hoofdstuk } 4}$ is de type-specifieke VE gemeten tegen anale HPV. De VE was hoog tegen de vaccintypes HPV16/18 (90\%, 95\% BI 63\%-97\%). Daarnaast was er significante kruisbescherming tegen HPV45 (VE=100\%) en tegen HPV31 (VE=73\%). De type-specifieke VE tegen anale HPV correleerde goed met de VE tegen genitale HPV (Spearman's $\rho=0,76$ ), wat wijst op vergelijkbare effectiviteit tegen anale als tegen genitale HPV infectie.

\section{Deel 2: Populatie impact op het voorkomen van HPV}

In het tweede deel van dit proefschrift wordt de impact van het HPV vaccinatieprogramma onderzocht op de prevalentie van HPV in de populatie. Hiervoor werden de gegevens van de PASSYON studie gebruikt. In Hoofdstuk 5 zijn de trends over tijd in de prevalentie van HPV16 en HPV18 onderzocht, tot 6 jaar na de invoering van vaccinatie. De HPV16/18 prevalentie daalde bij vrouwen (van 23\% in 2009 naar 15\% in 2015) en heteroseksuele mannen (van 17\% in 2009 naar 11\% in 2015), maar niet bij ongevaccineerde vrouwen. Bij heteroseksuele mannen wiens vaste partner in aanmerking was gekomen voor HPV vaccinatie, was de HPV16/18 prevalentie lager dan bij heteroseksuele mannen wiens vaste partner niet in aanmerking was gekomen voor HPV vaccinatie. De resultaten wijzen op groepsbescherming bij heteroseksuele mannen door HPV vaccinatie van meisjes. In Hoofdstuk 6 zijn de analyses van Hoofdstuk 5 uitgebreid met gegevens van 25 HPV types en tot 8 jaar na vaccinatie. In de periode 2009-2017 daalde de HPV16/18 prevalentie ieder jaar met $12,6 \%$ bij vrouwen en met $13,0 \%$ bij heteroseksuele mannen. Met data tot 8 jaar na vaccinatie daalde de HPV16/18 prevalentie ook bij ongevaccineerde vrouwen (met 5,4\% per jaar). Daarnaast daalde de HPV31 en HPV45 prevalentie bij vrouwen en heteroseksuele mannen en steeg de HPV56 prevalentie bij vrouwen en de HPV52 prevalentie bij ongevaccineerde vrouwen. De resultaten wijzen op groepsbescherming bij heteroseksuele mannen voor HPV16/18 en de HPV types waarvoor kruisbescherming is aangetoond. Bovendien wijzen de resultaten erop dat er ook groepsbescherming is voor de vaccintypes bij ongevaccineerde vrouwen. In $\underline{\text { Hoofdstuk } 7}$ hebben we onderzoek gedaan naar mogelijke groepsbescherming bij mannen die seks hebben met mannen (MSM). In de periode 20092017 was er geen daling in de HPV prevalentie bij MSM. Dit wijst erop dat MSM niet of nauwelijks baat hebben bij HPV vaccinatie van meisjes. Daarnaast hebben we in dit hoofdstuk gekeken naar eerdere blootstelling aan HPV bij MSM, door de aanwezigheid van HPV DNA op de penis en in de anus, en de aanwezigheid van HPV antistoffen te bestuderen. 
Het merendeel van de MSM testte negatief voor HPV DNA; 79\% testte negatief voor alle types in het $2 \mathrm{vHPV}$ vaccin, $62 \%$ testte negatief voor alle types in het $4 \mathrm{vHPV}$ vaccin, en $53 \%$ testte negatief voor alle types in het 9vHPV vaccin. Van de MSM die negatief testten voor HPV16/18 en HPV16/18/31/33/45/52/58 DNA, had 82\% respectievelijk 71\% ook geen meetbare HPV antistoffen tegen deze types. Omdat veel MSM negatief testten voor HPV DNA en HPV antistoffen, zou HPV vaccinatie van jonge MSM die de CSG bezoeken zinvol kunnen zijn.

\section{Deel 3: Directe vaccinatie effectiviteit tegen anogenitale wratten}

In deel 3 van dit proefschrift wordt de VE tegen AGW onderzocht binnen vrouwen die in aanmerking kwamen voor HPV vaccinatie in Nederland. In Hoofdstuk 8 is hiervoor gebruik gemaakt van de PASSYON studie. We hebben gekeken naar het effect van het $2 \mathrm{vHPV}$ vaccin tegen AGW en tegen genitale HPV6/11, de belangrijkste types die AGW veroorzaken. In deze studie was er geen effect van 2vHPV vaccinatie tegen HPV6/11 (VE=9\%, 95\% BI -31\%$37 \%$ ). Het effect van $2 \mathrm{VHPV}$ vaccinatie tegen AGW was niet statistisch significant (VE=36\%, 95\% BI -107\%-78\%). In Hoofdstuk 9 hebben we onderzoek gedaan naar het effect van 2vHPV vaccinatie tegen AGW door gebruik te maken van huisartsendata, omdat hier verreweg de meeste AGW diagnoses worden gesteld in Nederland. Door de gegevens van huisartsen te koppelen aan het Nederlandse vaccinatieregister, konden we de AGW incidentie vergelijken tussen 2vHPV gevaccineerde en niet-gevaccineerde vrouwen. The AGW incidentie was lager na $\geq 1$ dosis vaccinatie ten opzichte van geen vaccinatie (VE $=25 \%$, $95 \% \mathrm{BI} 12 \%-36 \%$ ). De VE was groter na volledige vaccinatie met 2 of 3 doses (VE=28\%) en wanneer de vrouwen 12-13 jaar oud waren op het moment dat ze gevaccineerd werden (VE=31\%). Hoewel we in deze studie niet konden corrigeren voor seksueel risicogedrag, lijkt het op basis van deze resultaten dat 2vHPV vaccinatie deels bescherming biedt tegen AGW.

Tot slot, in Hoofdstuk 10 worden de belangrijkste resultaten van dit proefschrift samengevat en bediscussieerd. Daarnaast werpt dit hoofdstuk een blik op de toekomst voor het meten van individuele en populatie-effecten van HPV vaccinatie, rekening houdend met de aanstaande veranderingen in het HPV vaccinatieprogramma, zoals het verlagen van de leeftijd van de start van vaccinatie, vaccinatie van zowel jongens als meisjes, en de mogelijkheid tot vaccinatie op latere leeftijd. De studies in dit proefschrift dragen bij aan de wetenschappelijke onderbouwing van de effectiviteit van het HPV vaccinatieprogramma, zowel op individueel als op populatieniveau. De PASSYON studie is waardevol gebleken in het meten van verschillende vaccinatie-effecten van HPV vaccinatie en kan ook in de toekomst waardevol zijn in het meten van de effecten van veranderingen in het programma. 


\section{Valorization}

Of all infectious diseases, human papillomavirus (HPV) infections are associated with the highest disease burden in the Netherlands. Sexually transmitted HPV can cause anogenital warts, recurrent respiratory papillomatosis, and various anogenital and head-and-neck cancers in both men and women. Cancers with an established HPV etiology include cervical, vaginal, vulvar, anal, and oropharyngeal cancer in women; and penile, anal, and oropharyngeal cancer in men. The estimated yearly number of cancer diagnoses attributed to HPV in the Netherlands was over twelve hundred in the period 2008-2017 and almost fifteen hundred in 2019. The estimated yearly number of anogenital warts diagnoses was over forty-three thousand in the period 2017-2018. Sexually transmitted HPV infections are very prevalent in the general population and the vast majority of the sexually active people will acquire an HPV infection at least once during lifetime. Infections are not restricted to specific groups at high risk of infection, making the prevention of HPV infections of public health importance. Prophylactic vaccination is viewed to be best possible prevention strategy and historically vaccination programs have achieved major public health benefits.

Prophylactic HPV vaccination was introduced in the Netherlands in 2009 for young girls using the bivalent HPV vaccine that targets the most oncogenic types HPV16 and HPV18. Each year all 12-year-old girls are invited for HPV vaccination. From 2021 onwards, even the entire cohort of boys and girls will be invited for HPV vaccination annually. This highlights that the effects of HPV vaccination are of societal relevance. In this thesis, we assessed the individual- as well as the population-level effects of HPV vaccination on HPV positivity and anogenital warts.

At the time of HPV vaccine introduction in the Netherlands in 2009, there were some uncertainties regarding the effectiveness of HPV vaccination, for example about the direct vaccine effectiveness in a "real-life" setting among the target population of (pre)adolescents, the duration of protection, the level of cross-protection, or the possible occurrence of type-replacement. This thesis has given insight into many of these questions. Lessons learned from this thesis, that are relevant for public health, include:

- $\quad$ The vaccine effectiveness of bivalent HPV vaccination is high against the most oncogenic vaccine-targeted HPV types 16 and 18;

- There is relevant cross-protection against non-vaccine oncogenic HPV types;

- The vaccine effectiveness is high at least up to $7 / 8$ years after vaccination, with no signs of reduced effectiveness over time;

- The vaccine effectiveness against anal HPV positivity is comparably high as against genital HPV positivity; 
- The HPV vaccination program has reduced the transmission of HPV in the population, leading to less infections among those unvaccinated, with the exception of men who have sex with men;

- $\quad$ There are no indications for type-replacement up to 8 years after the introduction of the HPV vaccination program;

- The bivalent HPV vaccine might provide partial protection against anogenital warts.

The lessons learned in this thesis have already contributed to the intended changes in the HPV vaccination policy. The Dutch Health Council valued our research in recommending about the new HPV vaccination program. For example, the sustained high VE, was taken into consideration in the recommendation to lower the age at which vaccination should be offered from 12 to 9 years of age. Moreover, our finding that herd effects are measurable within 6 to 8 years after HPV vaccine introduction, was an important observation for the Health Council to recommend extension of the vaccination program to boys. They viewed our observed herd effects as evidence that adding boys to the program will not only result in the individual prevention of boys themselves, but will also add to the protection of unvaccinated women through herd protection.

The results of this thesis are also relevant for the choice of vaccine to be used in the HPV vaccination program, as demonstrated by the acknowledgement of our results in the tendering procedure of 2020 to contract an HPV vaccine for the restructured vaccination program starting in 2021. Up to date, there are 3 prophylactic vaccines that target HPV; a bivalent vaccine that targets the most oncogenic HPV types 16 and 18; a quadrivalent vaccine that targets HPV types 6 and 11 (that cause the majority of the anogenital warts) in addition to HPV types 16 and 18; a nonavalent vaccine that targets the oncogenic HPV types $31,33,45,52$, and 58 in addition to HPV types $16,18,6$, and 11 . The 3 vaccines are all licensed for men and women and indicated to prevent genital (cervical, vulvar, and vaginal) and anal (pre)cancers caused by the HPV vaccine types. The quadrivalent and nonavalent HPV vaccines are also indicated to prevent anogenital warts. The cross-protection of the bivalent vaccine against non-vaccine oncogenic HPV types as found in our studies is higher than that generally observed for the quadrivalent vaccine, making the bivalent vaccine superior in terms of cancer prevention. The cross-protection of the bivalent vaccine also diminishes the difference in cancer prevention between the bivalent and the nonavalent vaccine. The differences between the bivalent and nonavalent vaccine are even further diminished with the apparent partial protection of the bivalent HPV vaccine against anogenital warts. Taken together, the results of this thesis underscore the necessity to conduct proper cost-effectiveness analyses to compare the different HPV vaccines, taking into account cross-protection against the different HPV types and possible protection 
against anogenital warts. Because the HPV vaccination program is publicly funded, it is important to have good value for money.

By studying the individual- and population-level effects of HPV vaccination, we demonstrated the benefits of HPV vaccination. We estimated a vaccine effectiveness of $90 \%$ against the most oncogenic HPV types HPV16/18 and a reduction of $13 \%$ each year in the HPV16/18 prevalence among women and heterosexual men. With the observed crossprotection and protection against anal HPV, the benefits of bivalent HPV vaccination even go beyond initial indications of the registration of the vaccine. These results are useful to create public awareness about HPV infections and the importance of HPV vaccination, for example via media attention. These results are also useful to inform the general public and health care providers. Previous studies indicated that health care providers play an important role in the decision-making process to vaccinate. Informing both the general public and health care providers about the effectiveness of HPV vaccination, could possibly increase the HPV vaccination coverage. Currently, there is a lot of misinformation about HPV vaccination, mainly concerning side effects. Moreover, there are misperceptions about the effectiveness of HPV vaccination. The bivalent HPV vaccine that is used in the Netherlands might be conceived to protect only against the 2 HPV vaccine types, while broad cross-protection against multiple HPV types has been clearly demonstrated in our studies as well as in others. Another misperception is that HPV vaccination is believed to be ineffective once someone has had sexual intercourse. Our results show it is not necessarily too late to vaccinate after sexual debut. Even in a highly sexually active population of visitors of sexual health centers, many individuals seem to be still unexposed to the HPV vaccine types, indicating the vaccine effectiveness could be high.

The results of this thesis give rise to targeted HPV vaccination of men who have sex with men (MSM) visiting sexual health centers, as we showed that MSM have not yet benefitted from the girls-only vaccination program to a large extent and many MSM seem to be susceptible to HPV when visiting the sexual health center. An HPV vaccination program targeting MSM has the potential to have a major impact on the HPV prevalence among MSM who are at high risk for anal and oropharyngeal cancer; with the current girls-only HPV vaccination program about 566 life years are lost due to vaccine-preventable HPVrelated cancer among MSM each year. However, the HPV vaccine effectiveness in the target population of MSM visiting sexual health centers is unknown. This thesis has contributed to an innovative ZonMw research proposal to assess the vaccine effectiveness among MSM aged $\leq 26$ years who visit the sexual health center of Amsterdam, HPV-ECSTASE (Human Papillomavirus Vaccine EffeCtiveness STudy Among men who have SEx with men). Results of this study can inform policy on the desirability to roll out a targeted vaccination program nationally. 
Next to the lessons learned about the vaccine effectiveness among women and the population-level impact of the current girls-only HPV vaccination program, we also provided a framework to monitor the effects of the upcoming changes in the HPV vaccination program. Because in the PASSYON study the HPV prevalence is measured among both men and women, the PASSYON study can be used the measure the vaccine effectiveness among men after sex-neutral vaccination is implemented. Moreover, in the PASSYON study there is information available of the HPV prevalence from pre- girls-only vaccination and pre- sexneutral vaccination, enabling to study the additional population-level impact after switching to a sex-neutral vaccination program. With the unique design of the PASSYON study, different effects of the upcoming changes in the HPV vaccination program can be studied. In close collaboration with policy makers and observations from other studies, this research can direct the future of the HPV vaccination program in the Netherlands. 


\section{List of abbreviations}

\begin{tabular}{|c|c|}
\hline $2 v$ & Bivalent \\
\hline $4 v$ & Quadrivalent \\
\hline $9 v$ & Nonavalent \\
\hline AA & Amino acid \\
\hline ATC & Anatomical Therapeutic Chemical Classification System \\
\hline AGW & Anogenital wart \\
\hline aIRR & Adjusted incidence rate ratio \\
\hline $\mathrm{aOR}$ & Adjusted odds ratio \\
\hline aPR & Adjusted prevalence ratio \\
\hline AUC & Area under the curve \\
\hline $\mathrm{BP}$ & Buffer period \\
\hline $\mathrm{Cl}$ & Confidence interval \\
\hline CIN & Cervical intraepithelial neoplasia \\
\hline DALY & Disability-adjusted life-year \\
\hline DEIA & DNA enzymelinked immunoassay \\
\hline GEE & Generalized estimating equation \\
\hline GP & General practitioner \\
\hline HPV & Human papillomavirus \\
\hline $\mathrm{Hr}$ & High-risk \\
\hline ID & Identifier \\
\hline ICPC & International Classification of Primary Care \\
\hline ITT & Intention-to-treat \\
\hline LCR & Long control region \\
\hline LiPA & Line probe assay \\
\hline $\mathrm{Lr}$ & Low-risk \\
\hline LU & Luminex Units \\
\hline MSM & Men who have sex with men \\
\hline NIP & National immunization program \\
\hline Nivel-PCD & Nivel Primary Care Database \\
\hline OR & Odds ratio \\
\hline PASSYON & $\begin{array}{l}\text { PApillomavirus Surveillance among STI clinic YOungsters in the } \\
\text { Netherlands }\end{array}$ \\
\hline PCR & Polymerase chain reaction \\
\hline PP & Per-protocol \\
\hline PR & Prevalence ratio \\
\hline
\end{tabular}




$\begin{array}{ll}\text { Præventis } & \text { National immunization registry } \\ \text { RCT } & \text { Randomized controlled trial } \\ \text { ROC } & \text { Receiver operating characteristic } \\ \text { SHC } & \text { Sexual health center } \\ \text { STI } & \text { Sexually transmitted infection } \\ \text { VE } & \text { Direct vaccine effectiveness } \\ \text { VLP } & \text { Virus-like particle } \\ \text { WGS } & \text { Whole-genome sequences }\end{array}$




\section{Curriculum vitae}

Petra Woestenberg was born on the 12th of May, 1989 in Alphen (NB), the Neterlands. In 2007 she graduated from high school at Mill-Hill college in Goirle after which she started studying Biomedical Sciences in Nijmegen with a specialization in Epidemiology and Health Technology Assessment (HTA). During the master Biomedical Sciences, Petra developed great interest in infectious disease control. She did her epidemiology internship at the RIVM Center for Infectious Disease Control about the timeliness of the first DTaP-IPV vaccination among preterm infants, and her HTA internship also at the RIVM Center for Infectious Disease Control about the cost-effectiveness of vaccination during an influenza pandemic. Petra graduated with honor (cum laude) for her master's Biomedical Sciences in 2013. Following this, Petra started working as a junior epidemiologist at the RIVM doing research on several topics regarding the epidemiology of sexually transmitted infections in the Netherlands. In 2016 she started her PhD project at the RIVM Center for Infectious Disease Control in collaboration with Maastricht University, under the supervision of Dr. Hans Bogaards, Dr. Birgit van Benthem, and Prof. Dr. Christian Hoebe. The topic of her PhD project was "measuring the effects of HPV16/18 vaccination on HPV positivity and anogenital warts". During her PhD trajectory, Petra worked part time for a few months as a scientific secretary for the Dutch Health Council on the updated advice on HPV vaccination in the Netherlands. 


\section{List of publications}

Donken R, Hoes J, Knol MJ, Ogilvie GS, Dobson S, King AJ, Singer J, Woestenberg PJ, Bogaards JA, Meijer CJLM, de Melker HE. Measuring vaccine effectiveness against persistent HPV infections: a comparison of different statistical approaches. BMC Infect Dis. 2020; $20: 482$.

Woestenberg PJ, Guevara Morel AE, Bogaards JA, Hooiveld M, van 't Klooster TMS, Hoebe CJPA, van der Sande MAB, van Benthem BHB. Partial protective effect of bivalent HPV16/18 vaccination against anogenital warts in a large cohort of Dutch primary care patients. Clin Infect Dis. 2020; 17:ciaa582.

Woestenberg PJ, van Benthem BH, Bogaards JA, King AJ, van der Klis FR, Pasmans $H$, Leussink S, van der Sande MA, Hoebe CJ. HPV infections among young MSM visiting sexual health centers in the Netherlands: Opportunities for targeted HPV vaccination. Vaccine. 2020; 38:3321-3329.

Woestenberg PJ, King AJ, Van Benthem BH, Leussink S, Van der Sande MA, Hoebe CJ, Bogaards JA. Bivalent Vaccine Effectiveness Against Anal Human Papillomavirus Positivity Among Female Sexually Transmitted Infection Clinic Visitors in the Netherlands. J Infect Dis. 2020; 221:1280-1285.

Drolet M, Bénard É, Pérez N, Brisson M; HPV Vaccination Impact Study Group. Populationlevel impact and herd effects following the introduction of human papillomavirus vaccination programmes: updated systematic review and meta-analysis. Lancet. 2019; 394:497-509.

Bogaards JA, van der Weele $\mathrm{P}$, Woestenberg PJ, van Benthem BH, King AJ. Bivalent Human Papillomavirus (HPV) Vaccine Effectiveness Correlates With Phylogenetic Distance From HPV Vaccine Types 16 and 18. J Infect Dis. 2019; 220:1141-1146.

Woestenberg PJ, Bogaards JA, King AJ, Leussink S, van der Sande MA, Hoebe CJ, van Benthem $\mathrm{BH}$. Assessment of herd effects among women and heterosexual men after girlsonly HPV16/18 vaccination in the Netherlands: A repeated cross-sectional study. Int J Cancer. 2019; 144:2718-2727.

Donken R, King AJ, Bogaards JA, Woestenberg PJ, Meijer CJ, de Melker HE. High Effectiveness of the Bivalent Human Papillomavirus (HPV) Vaccine Against Incident and 
Persistent HPV Infections up to 6 Years After Vaccination in Young Dutch Women. J Infect Dis. 2018; 217:1579-1589.

Woestenberg PJ, King AJ, van Benthem BH, Donken R, Leussink S, van der Klis FR, de Melker $\mathrm{HE}$, van der Sande MA, Hoebe CJ, Bogaards JA. Bivalent Vaccine Effectiveness Against TypeSpecific HPV Positivity: Evidence for Cross-Protection Against Oncogenic Types Among Dutch STI Clinic Visitors. J Infect Dis. 2018; 217:213-222.

Woestenberg PJ, King AJ, van der Sande MA, Donken R, Leussink S, van der Klis FR, Hoebe $\mathrm{CJ}$, Bogaards JA, van Benthem BH. No evidence for cross-protection of the HPV-16/18 vaccine against HPV-6/11 positivity in female STI clinic visitors. J Infect. 2017; 74:393-400.

van Oeffelen AA, van den Broek IV, Doesburg M, Boogmans B, Götz HM, van LeeuwenVoerman FA, van Veen MG, Woestenberg PJ, van Benthem BH, van Steenbergen JE. Ethnic and regional differences in STI clinic use: a Dutch epidemiological study using aggregated STI clinic data combined with population numbers. Sex Transm Infect. 2017; 93:46-51.

Woestenberg PJ, Tjhie JH, de Melker HE, van der Klis FR, van Bergen JE, van der Sande MA, van Benthem $\mathrm{BH}$. Herpes simplex virus type 1 and type 2 in the Netherlands: seroprevalence, risk factors and changes during a 12-year period. BMC Infect Dis. 2016; $16: 364$.

Verscheijden MM*, Woestenberg PJ*, Götz HM, van Veen MG, Koedijk FD, van Benthem $\mathrm{BH}$. Sexually transmitted infections among female sex workers tested at STI clinics in the Netherlands, 2006-2013. Emerg Themes Epidemiol. 2015; 12:12.

Woestenberg PJ, van Oeffelen AA, Stirbu-Wagner I, van Benthem BH, van Bergen JE, van den Broek IV. Comparison of STI-related consultations among ethnic groups in the Netherlands: an epidemiologic study using electronic records from general practices. BMC Fam Pract. 2015; 16:70.

Woestenberg PJ, van Lier A, van der Maas NA, Drijfhout IH, Oomen PJ, de Melker HE. Delayed start of diphtheria, tetanus, acellular pertussis and inactivated polio vaccination in preterm and low birth weight infants in the Netherlands. Pediatr Infect Dis J. 2014; 33:1908. 
